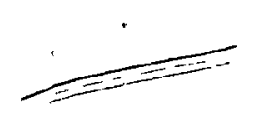

DETERMINATION OF CRUSTAL INTERFACE TOPOGRAPHY

FROM POTENTIAL EIELDS

\title{
by
}

\section{Mark Pilkington.}

\begin{abstract}
A thesis submitted to the Faculty. of Graduate studies and Research, McGill University, in partial fulfillment

of the requirements for the degree of Doctor of Philosophy.
\end{abstract}

\section{Dept. of Geological Sciences} McGill University, Montreal 
A method is presented for the determination of interface topography of multi-layered crustal models from gravity or magnetic data. The technique is developed within the framework of discrete linear inverse theory and exploits the quasi-linearity of the problem to derive a simple cost-effective algorithm suitable for use on large gridded data sets.

Variation of auxiliary parameters allows a suite of acceptable models to be produced rapidly and appraised in the light of available geological and geophysical evidence. When independent knowledge concerning the behaviour of specified interfaces is available, the incorporation of 'such data in the form of linear equality constraints ig outlined. The proposed method is applied to Curie isotherm and Moho mapping in the Abitibi greenstone belt. 


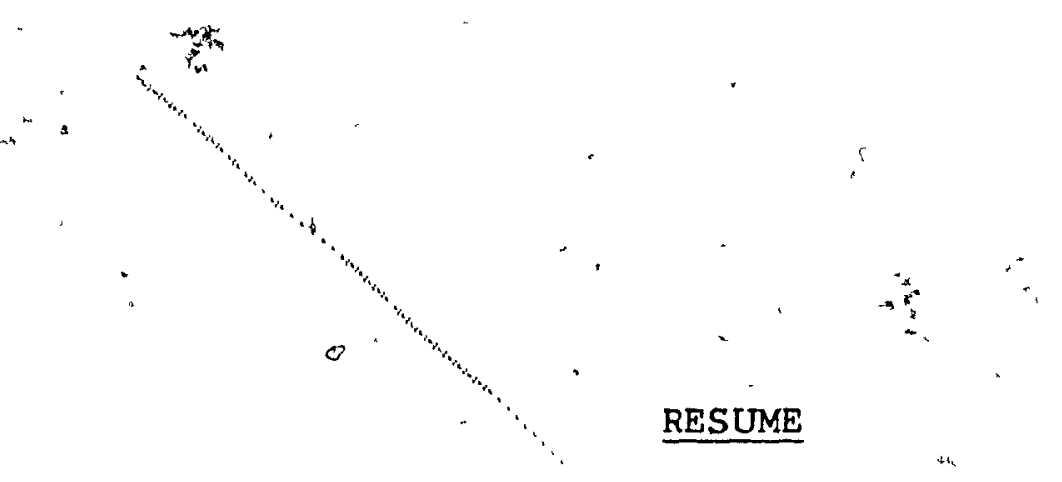

On présente une methode pour determiner la topographie d'interface des models à couches multiples de la croûte terreste à partir des donrées de gravité ou magnetiques. La technique a été developée dans la cadre de "discrete linear inverse theory" et profite de la quasi-linearité du problem pour trouver un algorithme simpile et économique pouvant être utilisé sur de nombreux données cadrillées.

La variation des paramètres auxiliaires permet de construire rapidement des models acceptables, et de les

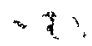
évaluer en tenant compte des preuves géologiques et géophysiques disponibles.

Lorsque des informations independentes concernant le comportment des interfaces specifées sont disponibles, l'incorporation de telíles données dans la forme des contraintes d'égalité lineaire a été exposée.

La methode a' été appliquée à l'isotherme de carie et au tracé de Moho dans la zone de roches vertes en Abitibi." 


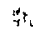

TABLE OF CONTENTS

CHAPTER 1

Page

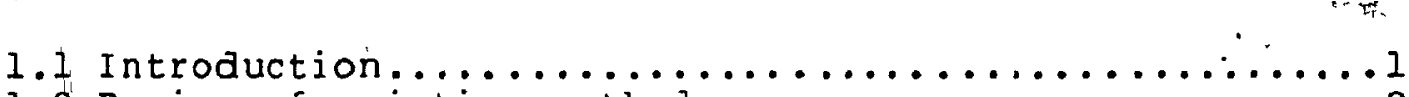

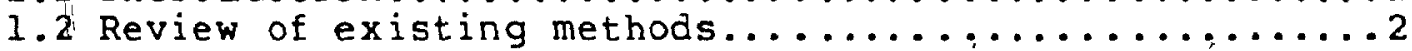

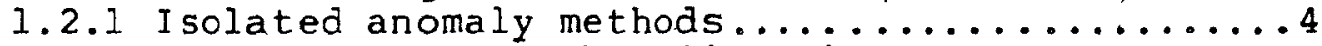

1.2.1.1 Naudy matched filtering............5

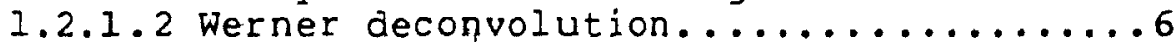

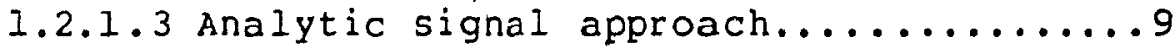

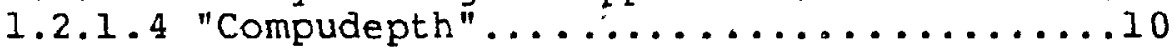

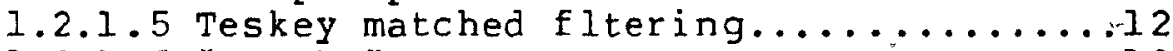

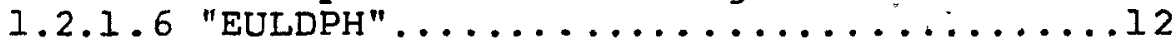

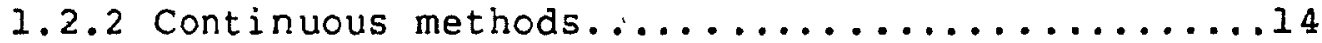

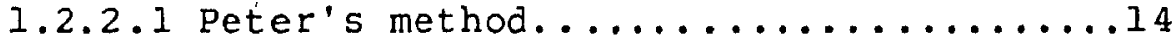

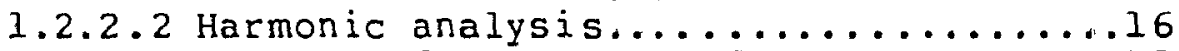

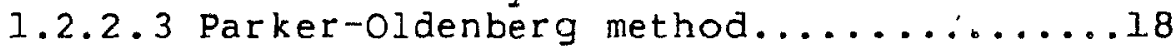

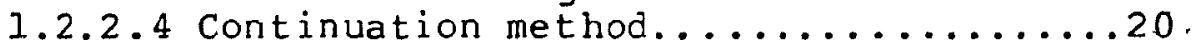

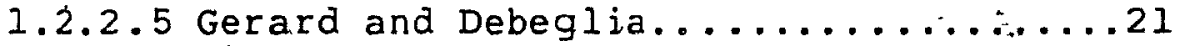

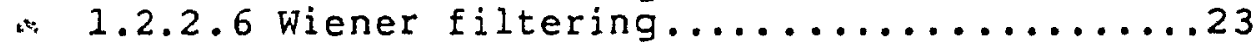

1.2.2.7 Generalized linear inverse approaches..24

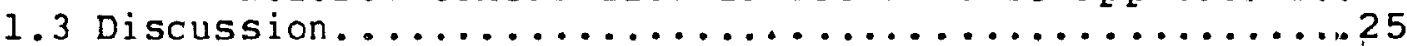

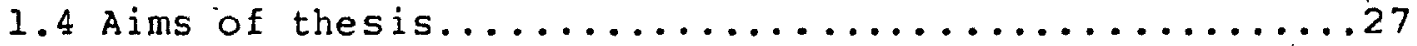

CHAPTER 2

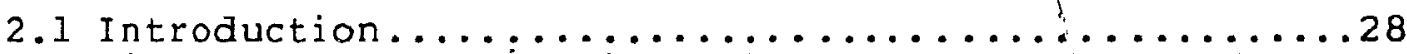

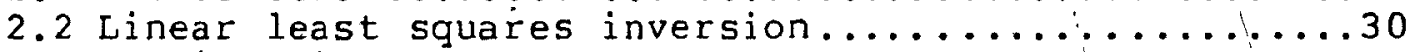

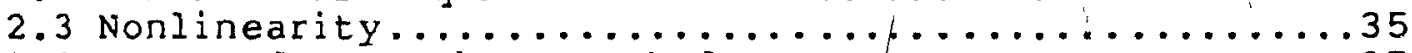

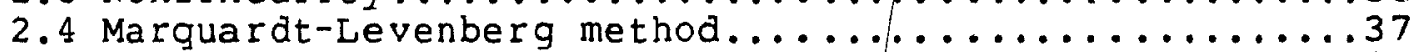

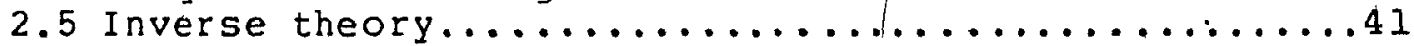

2.5 .1 Backus-Gilbert theory.................

2.5 .2 Singular value decomposition................44

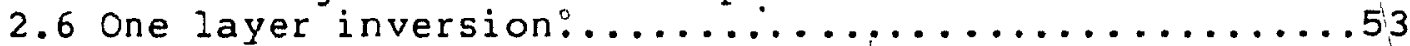

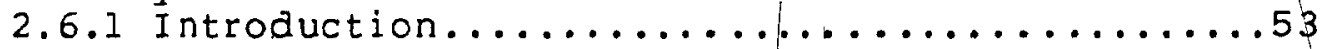

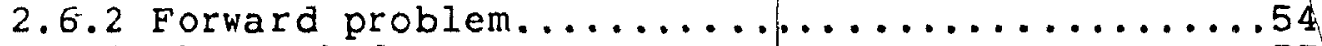

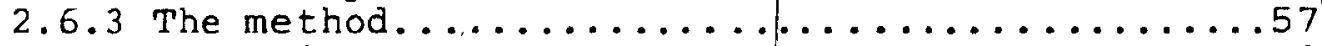

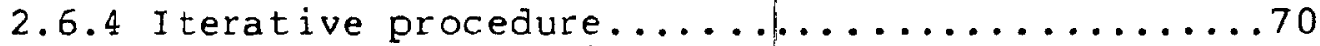

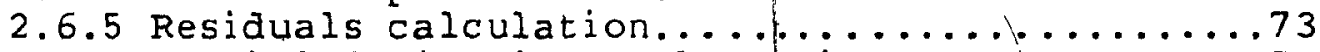

2.6.6 Partial derivatives calculation............74

2.7 Multilayer inversion.......................

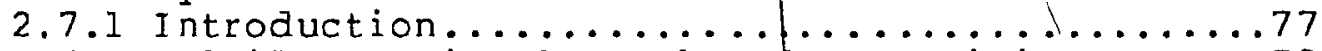

2.7.2 Multilayer singular value qecomposition.......78

2.7.3 Reparameterization and problem eigenvalues....88

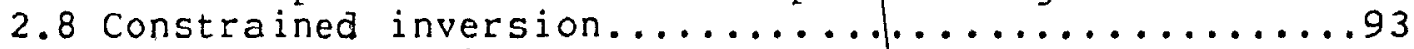

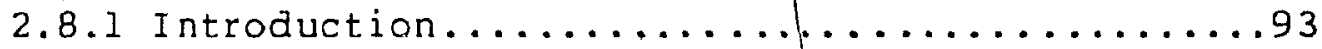

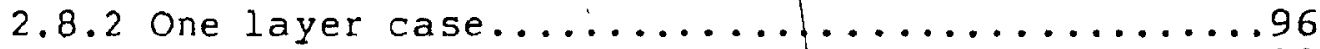

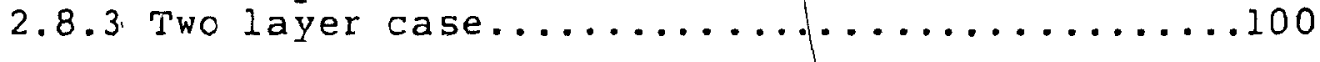


$\therefore$.

CHAPTER 3

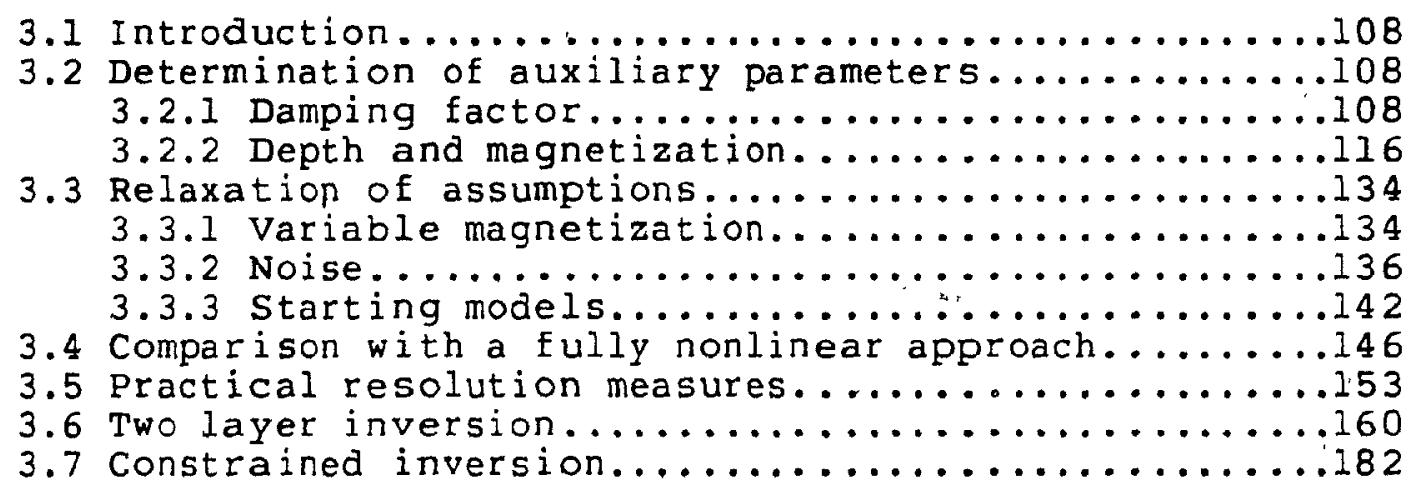

CHAPTER 4

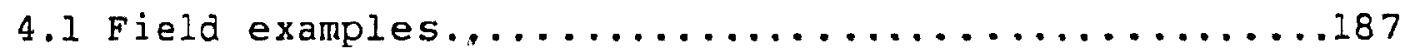

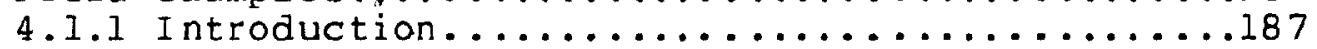

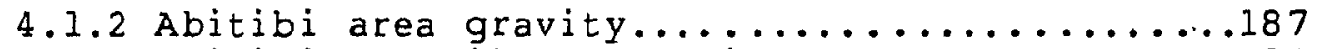

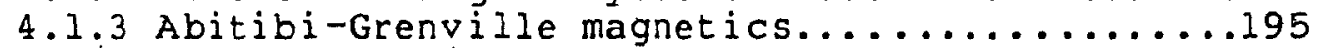

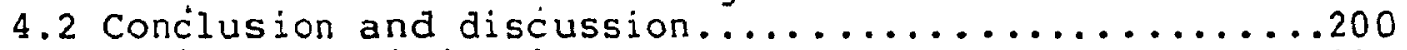

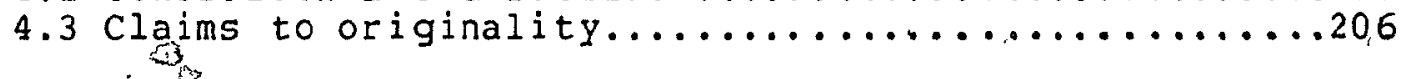

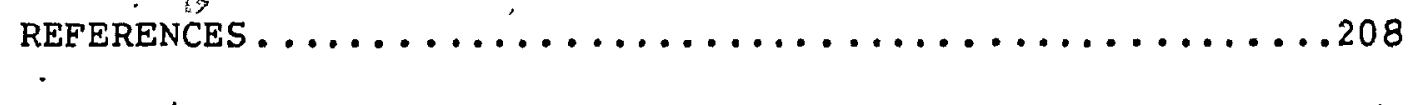


2.1 Generall form of trade-off diagram " 45

2.2 Eigenvalue spectra for the one layer problem as a Eunction of $\operatorname{depth(z)}$ for a layer with a magnetization of $1.2 \mathrm{~A} / \mathrm{m}$.

(a) Resolving kernels calculated from (2.6.3.9) for a layer with vertical magnetization of $1.2 \mathrm{~A} / \mathrm{m}$ as a function of depth. (b) Resolving kernels calculated from (2.6.3.9) for a layer at depth 6 units as a Eunction of magnetization.

2.4 Resolving kernels calculated from (2.6.3.9) for a layer with vertical magnetization of $1.2 \mathrm{~A} / \mathrm{m}$ and depth 6 units as a function of damping factor $\theta$.

2.5 . Resolving kernels for the two layer problem. Layer depths and magnetizations are $1.5,6.0$ arbitrary units and $0.6,1.2 \mathrm{~A} / \mathrm{m}$ respectively.

2.6. Resolving kernels for the two layer problem.
Layer depths and magnetizations are $3.0,3.5$ arbitrary units and $0.6,1.2 \mathrm{~A} / \mathrm{m}$ respectively.

2.7 Reparameterization in the wavenumber domain for the two layer problem. The ratios $F / V\left(F^{2}+G^{2}\right)$ and $G / V\left(F^{2}+G^{2}\right.$ ) (equation $(2.7 .2 .7)$ ) are plotted for the upper and lower layers respectively. In (a) the layer magnetizations are equal. In (b) the lower layer magnetization is 10 times that of the upper layer. In both (a) and (b) the dependence of the reparameterization on the distance between layers (separation) is illustrated.

2.8 Eigenvalue spectra for several two layer cases where $z l$ and $z 2$ give the layer depths in arbitrary units. The value $V\left(F^{2}+G^{2}\right)$ of $(2.7 .2 .7)$ is plotted. Magnetization is $0.6 \mathrm{~A} / \mathrm{m}$ for top layer and $1.2 \mathrm{~A} / \mathrm{m}$ for bottom. 
3.2.1 Ratio of inverse and damped inverse eigenvalues for a layer at depth 6 units and magnetization $1.2 \mathrm{~A} / \mathrm{m}$. Values of ID indicate the number of eigenvalue used as a damping factor.

111

3.2.2 Effect of damping on the inverse eigenvalue spectrum. Damped spectrum was produced by. a damping factor equal to the fourth eigenvalue.

3.2.3 (a) Model LI topography and calculated effect. Average layer depth is 5.26 arbitrary units with a magnetization of $1.2 \mathrm{~A} / \mathrm{m}$. (b) Model L2 topography and calculated effect. Average layer depth is 6 arbitrary units with a magnetization of $1.2 \mathrm{~A} / \mathrm{m}$.

3.2.4 Effect of damping on number of iterations required to reduce misfit to less than InT $^{2}$ for model L2.

3.2.5 Contour map of RMS error for model Ll.

3.2.6 Representative topograhies of the form $h(x)=$ $A a^{2} /\left(a^{2}+x^{2}\right)$ where the amplitude $A$ and half-width a are given.

3.2.7 Total field produced by models for which the product of magnetization and topography is constant.

124

3.2.8 Results of inverting model L2 for various magnetization values.

3.2.9 Dependence of RMS error on magnetization for the FLAT of model L2. The three curves correspond to different estimated depths given by the value of $z$ in the figure.

3.2.10 Results of inverting model L2 for various layer depths. Average layer depth ranges from 1 to 9 units.

3.2.11 Result's of Monte-Carlo search in depthsusceptibility space for model I2. Each acceptable model is shown as a square. The circle denotes the true values from which L2 data was generated.

3.2.12 Dependence of RMS error on depth for the ELAT of model L2. The three curves correspond to different estimated magnetizations given by the value of $m$ in the $f i g u r e$. 

3.3.I. Effects of variable magnetization. Solutions
for the magnetization distributions AMI, AM2 and AM3 (see text) are given in a. Difference between estimates in a. and the true solution are given in $b$.

3.3 .2 . Effects of noisy data. Black line in (a) denotes noise-free inversion. Coloured. I ines are inversions for damping factors given by $D$. White noise sample added to data is shown in (b). The final RMS error: for inversions is given by, the value of rms.

3.3 .3 Effects of noisy data. Black line in (a) denotes noise-free inversion. Coloured $I$ ines are inversions for damping factors given by $D$. White noise sample added to data is shown in (b). The final RMS error for inversions is given by the value of rms.

3.3.4 Effects of long wavelength noise on inversion. Black line in (a) denotes noise-free inversion. Coloured Iines are inversions for damping factors given by $D$. Noise sample is shown in (b).

3.3.5 Effects of starting models. Inversions of model L2 are given in (a) with the corresponding starting model in (b).

3.3.6 Effects of starting models. Inversions of model I2 are given in (a) with the corresponding starting model in (b).

3.3.7 Effects of starting models. Inversions of model

L2 are given in (a) with the corresponding starting model in (b).

3.4.1 Comparison of model and computed (for varying $\theta)$ solution amplitude spectra for model L2.

3.4.2 Comparison of model and computed solutions 149 $(\theta=17.6)$ for model L2.

3.4.3 Results of inversion of model L2 data from a
a nonlinear inversion programme (curve SvD) and the proposed method (curve TOPO) for a damping factor of 50.4 .

3.4.4 Comparison of iterative behaviour of RMS error for programmes SVD and TOPO. 
3.5.1 True and approximate resolving kernels for - 159 , topography estimates at distances 9 and 15 units.

3.5.2 True and approximate resolving kernels for 161 topography estimates at distances 9 and 15 units.

3.6.1 Upper layer inverse eigenvalue spectra (equation(3.9a)) for varying layer configurations. Magnetization is $0.6 \mathrm{~A} / \mathrm{m}$ for top layer and $1.2 \mathrm{~A} / \mathrm{m}$ for bottom.

3.6.2 Lower layer inverse eigenvalue spectra (equation(3.9b)) for varying layer conftgurations. Magnetization is $0.6 \mathrm{~A} / \mathrm{m}$ for top layer and $1.2 \mathrm{~A} / \mathrm{m}$ for bottom.

3.6.3 Model LL1. Lower layer magnetization is $1.2 \mathrm{~A} / \mathrm{m} \quad 166$ Upper layer magnetization is $0.6 \mathrm{~A} / \mathrm{m}$.

3.6.4 Model LLI and calculated solution.

3.6.5 Starting models and results from the proposed 169 method (curve TOPO) and a generalized nonl inear inversion programme (curve SVD) for model LLI data.

3.6.6 Starting models and results from the proposed method (curve TOPO) and a generalized nonlinear inversion programme (curve SVD) for model LL' data.

3.6.7 Model LL2. Lower layer magnetization is $3.0 \mathrm{~A} / \mathrm{m} \quad 173$ Upper layer magnetization is $0.6 \mathrm{~A} / \mathrm{m}$.

3.6.8 Model LL2 and calculated solution. 174

3.6.9 Model LL3. Lower layer magnetization is $1.2 \mathrm{~A} / \mathrm{m} .175$ Upper wayer magnetization is $0.6 \mathrm{~A} / \mathrm{m}$.

3:6.10 Model LL3 and calculated solutions for noisy 176 and noisé-free data.

3.6.11 Model LL4. Lower layer magnetization is $1.2 \mathrm{~A} / \mathrm{m} \quad 177$ Upper layer magnetization is $1.2 \mathrm{~A} / \mathrm{m}$.

3:6.12 Model LI4 and calculated solution. 
3.6.13 One solution to the homogeneous problem (noplinear form of (3.10)). Average layer 180 depths are 2.0 and 5.0 units. Magnetization contrasts are $1.2 \mathrm{~A} / \mathrm{m}$ and $-1.2 \mathrm{~A} / \mathrm{m}$ for the upper and lower interfaces.

3.6.14 Another solution to the homogeneous problem (nonlinear form of $(3.10)$ ). Average layer depths are 2.0 and 5.0 units. Magnetization contrasts are $1.2 \mathrm{~A} / \mathrm{m}$ and $-1.2 \mathrm{~A} / \mathrm{m}$ for the upper and lower interfaces.

3.7.1 Constrained and unconstrained solutions for 184 model L2. Constrained parameter is marked by an arrow.

3.7.2 Constrained and unconstrained solutions for 185 model LII. Constrained parameters are marked by arrows.

4.1 Bouguer gravity map of study area. Contour 189 interval is $10 \mathrm{mgals}$. y

4.2 Simplified geological map of the Abitibi region 190 (after Ludden and Hubert, 1984).

4.3 Calculated variation in Moho topography. 192 Contour interval is $2 \mathrm{~km}$.

4.4 Gravity field produced by the model of Figure 194 4.3. Contour interval is $5 \mathrm{mgal}$.

4.5 Residual aeromagnetic Eield over the Abitibi- 196. Grenville region. Contoun interval is $100 \mathrm{nT}$.

4.6 Relief of maghêtiticrut bottom. Contour 199 interval is $3 \mathrm{~km}$

4.7 Total ifield produced by the model of Figure 201 4.6. Contour interval is 50nT. 


\section{IIST OF TABLES}

Table 2.1. Condition number of the Jacobian for a two .92 layer model with layer 2 at constant depth.

Table 2:2 Condition number of the Jacobian for a two layer model with layerl at constant depth.

Table 3.I. Maximum damped inverse eigenvalue as a 114 function of damping factor.

- Table 3.2 Summary of inversions for L'. Values of final RMS error are shown as a function of $\mathrm{J}$ and $z_{0}$. Divergent solutions are denoted by div.:

Table 3.3 Number of iterations required to reach an 150 acceptable solution (RMS error < InT') for model L2 using programmes' SVD and TOPO.

Table 3.4 True and approximate resolution measures for 158 the solution of model L2. 


\section{ACKNOWLEDGEMENTS}

The -author would 'Iike to acknowledge the support and guidance of Dr. D.J..Crossley throughout this work. Dr. E.J. Schwarz kindly made available the Abitibi magnetic and gravity datạ sets.

This research was supported by the National science and Engineering Council and by McGill University through the award of the James Douglas Fellowship. 


\section{CHAPTER I}

\section{I:I Introduction}

The primary purpose of magnetic exploration in the petroleum industry. is the determination of the depth to and the topography of the basement surface. The thickness of the sedimentary sequence overlying the basement is important from the point of view of conditions conducive to oil formation; the basement relief may show features controlling the structure of overlying sediments, possibly indicating oil-trapping environments. As the magnetite content, and hence the susceptibility, of basement rocks is so much greater than the sediments, the magnetic anomalies'observed over such areas are primarily the result of supra- and intrabasemental features.

From potential theory it is known that the sources producing a given potential field are not unique in nature. Hence, the measurement of the magnetic field over an area provides no: unique information regarding the distribution and size of the causative bodies. Fortunately, this ambiguity can be reduced by taking advantage of pertinent geologic information and data, from other geophysical methods 
to constrain the interpretation within realistic, bounds. The constraints usually take the form of restricting the geometry of the causative body to simple shapes such as prisms, cylinders etc. or to restricting the range of parameters involved (e.g. susceptibility). With this limitation in mind, aeromagnetic data can be used in the determination of crystaline basement topographies.

\section{$\underline{1} \cdot \underline{2}$ Review of existing methods}

Under the heading of basement mapping, one can include most existing methods of interpretation which involve estimation of source depth: these range from simple graphical methods to more complicated automated procedures introduced during the 1970's. The distinction is made here between two major approaches: the first involves isolated anomalies, the second cdnsiders complete (continuous) sections or areas of data containing numerous anomalies.

For the former case, the parameters determined from the interpretation of each of the isolated anomalies are used to contribute to the general picture of basement relief. The advantage of this approach is in selecting features that are free from the disturbing effects of adjacent anomalies and those that appear to be caused by geometrically simple bodies. Several automated procedures have been introduced (section $(1.2 .1)$ ) for this problem, "most of them applicable 
to profile (two-dimensional) data. In addition to the computations necessary to estimate physical parameters from anomalies, a.significant amount of effort must be expended on defining criteria for the detection and location of features of interest. Thus the interpreter's skill and judgement is quantified in a statistical or empirically derived decision criterion.

The latter (continuous) method of utilising all the data available for interpretation is relevant when the rather restrictive assumption can be made that all anomalies present are the result of lateral magnetization changes due to basement surface topography. Based on the strength of this assumption, it may be necessary to pre-process the data to remove the effect of shallow sources and any strong anomalies that can be identified as resulting from intrabasemental magnetization contrasts. Although in theory there is no way of distinguishing between relief anomalies and magnetization anomalies, it is usually found that relief anomalies are the weaker of the two (Nettleton, 1976, p.384). In contrast to the isolated anomaly approach which results in a distribution of individual depth estimates that are not necessarily from the same horizon, continuous methods result directly in a basement map that, usually assumes only one susceptibility interface produced directly from the data.

The, major difference between the two approaches is the 
stage at which the interpreter's judgement is decisive in forming a valid geological model. For isolated anomaly methods, this occurs both in the anomaly selection stage and later in combining the relevant parameter values into an overall geological model. For continuous methods, the burden falls on the interpreter to estimate some fixed, relevant parameters and sometimes to assume the form of the model prior to the use of the method. It may be argued that the assumptions made are too restrictive to be of any practical value. However, if the result of analysis is considered to be an initial step in the interpretive process subject to subsequent refinement, then such methods do provide useful information.

\subsubsection{Isolated anomaly methods}

The methods used for the analysis of single residual anomalies are too numerous to mention individually; the more important can be found both in the standard geophysical texts (e.g. Telford et al.,1976, Chap. 3.6) and review articles (Hinze, 1979). This section is limited to a discussion of automated approaches that have met with success as regards speed, cost, versatility and objectivity in interpretation. The methods primarily concern depth estimation although they may also produce estimates of other parameters. 


\subsubsection{Naudy matched filtering}

Naudy(1971) presented a two-part procedure based on matched filtering to determine depths, positions and magnetizations of sources present in raw (unprocessed) aeromagnetic data. The initial phase of detecting and locating anomalies is achieved by the use of a coefficient of similarity defined as

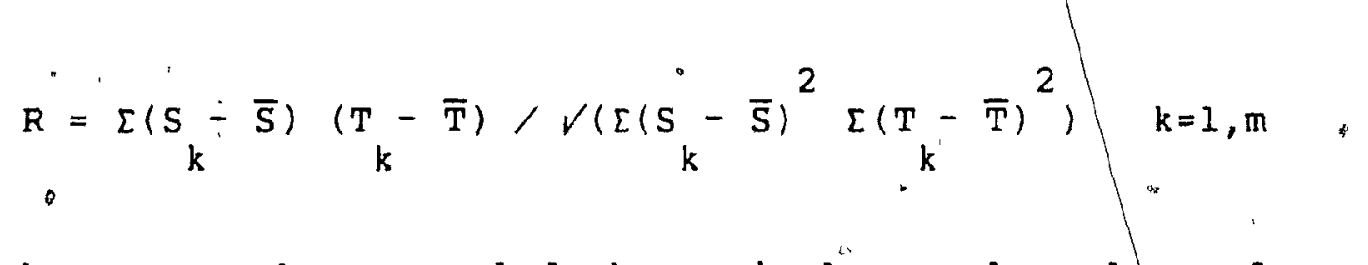

where the $T_{k}$ are the $m$ sampled theoretical" anomaly values of a vertical, infinite depth dyke with a mean value $\bar{T}$ over the $m$ samples and the $S_{k}$ are the values taken from the total field profiles. In order to account for the dip of the body and direction of magnetization the data are split into symmetrical and antisymmetrical components. symmetrica? curves correspond to the field over a vertically magnetized dyke while the antisymmetric curves relate to a dyke magnetized at $45^{\circ}$. The latter can be transformed using a pole reduction operator into the symmetrical form and both data sets processed simultaneously.

The sampled theoretical anomaly is then passed over the data and $R$ calculated at each data point. Repeated passes are made with several sampling intervals in order to sense sources at different depths. If an anomaly caused by a dyke of depth $d$ and width $b$ is sampled at an interval $s$, then 
expanding this interval to 25 gives the anomaly of a dyke with depth $2 d$, width $2 b$, and the same magnetization. The maximum values of $R$ give the location of the anomalies and from the sampling interval at which the "detection" occured, an initial estimate of the depth can be made.

The final part of the method is the relaxation of the theoretital anomaly source parameters, repealted application of the matched filters, and the calculation of the resulting R's, thus allowing a more precise determination of depths etc. Along with the usual assumptions for profile methods the matched filtering procedure is limited by the simplicity of the theoretical bodies used.

\subsubsection{Werner deconvolution}

Based on Werner's! (1953) expression for the total field produced by an arbitrarily magnetized thin dyke, Hartmann et al (1971) introduced an automatic method for profile aeromagnetic data. Because the horizontal gradient of the total field due to a geological contact is equivalent to the totalfield of a thin dyke, the number of models that the method responds to is doubled. The relationship between source parameters and observed field is linear; the total field at coordinates $\left(x_{0}, D\right)$ is given by

$$
T\left(x_{0}, D\right)=\frac{A\left(x_{0}-x\right)+B D}{\left(x_{0}-x\right)^{2}+D^{2}}
$$


where

$$
\begin{aligned}
& x=x \text { coordinate of dyke top } \\
& D=\text { depth to top of dyke } \\
& A=-2 \Delta T \text { (HsinI + vcosIsin }) \\
& B=2 \Delta T \text { (-Hcosisin }+ \text { VsinI) } \\
& I=\text { Geomagnetic inclination }
\end{aligned}
$$

$H, V=$ Horizontal and vertical magnetization components$$
\Delta \mathrm{T}=\text { Dyke thickness/2 }
$$$$
\cdot \beta=\text { Angle between magnetic north and direction }
$$$$
\text { normal to profile direction }
$$

Rewriting (1.1) in the form

$$
a_{0}+a_{1} x+b_{0} T+b_{2} x T=x^{2} T
$$

where

$$
\begin{aligned}
& a_{0}=-A x_{0}+B D \\
& a_{1}=A \\
& b_{0}=-x_{0}^{2}-D^{2} \\
& b_{1}=2 x_{0}
\end{aligned}
$$

allows the determination of the unknown $a_{0}, a_{1}, b_{0}, b_{1}$ (once the values of $T$ are known at four consecutive points) by solving the linear system of simultaneous equations (1.2). The computational labour, cạn be reduced by using a set of linear operators for the solution (Werner, 1953). The possibility of interference over the data window is accomodated by the addition of a. low order polynomial to (1.I) which increases the number of equations to $(n+5)$, where $n$ is the order of polynomial chosen. Hartmann et al 
suggest that $n \leq 2$ is sufficient for most practical cases. Kilty(1983) has shown that the type of polynomial assumed affects the sensistivity of the method to noise. For example, using a linear trend reduces the anomaly amplitude and information. is lost concerning the body magnetization. He also indicates that the method breaks down when more" than 3\% of random additive Gaussian white noise is present. Ku and sharp (1983) advocate an iterative technique for the remọval of interference effects.

The operator is then applied at intervals of one data point and the resulting estimates of the parameters as calculated from (1.1) and (1.2) are plotted by computer. To obtain the most accurate estimates, the operator should be roughly the same size as the anomaly modelled, thus to facilitate interpretation of anomalies arising from different source depth', the size of the operator is varied (the number of data points remaining constant). Both the total field, and its calculated horizontal gradient are processed leading to solutions for the dyke and contact models. The amount of scatter in the depth/location plots for these solutions give an indication of whether the assumed model is present; as usual the final judgement should incorporate other pertinent information.

Werner deconvolution has the advantage of being easy to implement, computationally cheap, incóporates regional/residual separation and exhibits good resolving 
power (Jain, 1976). Ku and Sharp (1983) have used the method to provide initial estimates for an iterative nonínear least squares inversion programme which relaxes the constraints imposed by the thin dyke and contact models.

\subsubsection{Analytic signal approach}

Nabighian (1972) introduced the concept of the analytical signal into the interpretation of magnetic profile data caused by structures assumed to be polygonal in cross-section. The analytic function $A(x)$ is determined by frequency domain methods: its real part is the horizontal derivative of the field and its imaginary part the vertical derivative. Thé latter can be calculated from the former by Hilbert transformation. Nabighian shows that the amplitude of $A(x)$ or any of its higher horizontal derivatives are symmetric functions with maxima occurring over body vertices, thus providing'a method for their location. Vertex depths are calculated from the half-width of the function $|A(x)|^{2}$. The determination of the remaining parameters is straightforward once the depth and positions of the vertices are known. The success of the procedure is dependent on locating the maxima of $|A(x)|^{2}$ and decomposing it into its symmetric parts when several edges are present: this is usually accomplished by visual inspection followed by a nonlineat least squares method to refine the initial \& guesses. The influence of noise and undesirable features in 
the data are reduced at this point by low-pass filtering, if this has not already been carried out earlier.

Further properties of the function $A(x)$ can be exploited to determine the source body shape (Nabighian, 1974): by taking successively higher derivatives of $A(x)$, depth differences of the vertices are enhanced. Needless to say, independent information is required to discard any features produced by noise amplification. Gunn (1979) has given further examples of this approach while Nabighian (1984) has extended his formalism to the three dimensional case.

\subsubsection{4 "Compudepth"}

O'Brien (1972) has developed a magnetic inversion programme called "Compudepth" for rapid quantitative interpretation of large blocks of profile data. The mothod is intended for preliminary investigation of geofogical structure for both oil and mineral exploration requirements rather than detailed modelling of individual anomalies required in developing mining sites.

The basis of the technique is the linear relation between source depth and the slope of the log-spectrum of the source field. Compudepth also uses the well known result that the horizontal derivative of the anomaly produced by a contact is equal to the anomaly produced by a thin dyke (Hartmann et a 1, 1971; Nabighian, 1972). If sources are presumed to be polygonal, they can be decomposed into a number of contacts 
(and therefore, dykes). The total field due to a thin dyke att. location $(x, 0)$ is given by

$$
T(x)=\frac{k_{1} \cdot z_{0}+k_{2}\left(x-x_{0}\right)}{\left(x-x_{0}\right)^{2}+z_{0}^{2}+\left(x-x_{0}\right)^{2}+z_{0}^{2}}
$$

where $x_{0}$ and $z_{0}$ are the coordinates of the top of the dyke. The Fourier transform of this field at wavenumber $u$ has the simple form

$$
T(u)=\left(k_{1}-i \operatorname{sgn}(u) k_{2}\right) e^{j \mid u / z_{0}} e^{-i u x_{0}}
$$

so that the real part gives the depth and the imaginary part the location. If a number of source depths are similar along a profile, the determination of these depths is equivalent to fitting a sum of exponentials to the profile transform; this is known to be a highly unstable problem (Twomey, 1977, p.172) so that recourse should be made to processing in the spatial domain to improve resolution. In practice, a moving Window of data is processed at a time reducing the number of depths to be determined. Using overlapping sets of data, the better determined depths are found repeatedly. Edge locations are initially found using this approach and improved using a nonlinear regression technique in the frequency domain similar to that of Bhattacharyya and Leu (1975). Similar to Nabighian's method (section (1.2.1.3)), "Compudepth" assumes only that the bodies modelled are of two-dimensional polygonal form. 
Gunn (1979) has given some examples of "compudepth". on real data.

\subsubsection{Teskey matched filtering}

Teskey (1978) has extended the approach of Naudy (1971). to determine strike direction and strike length of anomalies along with parameters defining source properties. The basis of the method is similar to Naudy's: sampled theoretical anomalies from a prism source are passed over the data with - the sample interval varied to accomodate a range source depths. The determination of strike is made by passing the matched filters in several directions over the gridded data and finding the orientation giving the maximum correlation. Strike length is estimated from the magnitude of the correlation when filters corresponding to various strike lengths are used. A simple statistical decision criterion is used to discard insignificant anomalies. Teskey's method produces a range of acceptable solutions that fit the data, leaving the final decision to the interpreter.

\section{2 .1 .6 "EULDPH"}

Thompson(1982) has developed an automated procedure ("EULDPH") for obtaining depfestimates from. gridded data using Euler's homogeneity relationship. The method has the. Eundamental advantage over existing techniques in that no geometric model has to be specified prior to analysis. The 
theoretical basis arises from many magnetic source fields, satisfying the Euler equation:

$$
x \frac{\partial f}{\partial x}+y \frac{\partial f}{\partial y}+z \frac{\partial f}{\partial z}=n f
$$

where the function $(x, y, z)$ is said to be homogeneous of degree $n$ if it satisfies (1.3): The structural index $N$ (= $-n)$ depends on the type of source and ranges from 1 for a line of poles to 3 for a point dipole. If the total field from a two-dimensional source is of the form $T(x)=f\left(\left(x-x_{0}\right), z_{0}\right)$, Euler's equation becomes

$$
\left(x-x_{0}\right) \frac{\partial T}{\partial x}-z_{0} \frac{\partial T}{\partial z}=N T(x)
$$

from which the source position $\left(x_{0}, z_{0}\right)$ and structural index can be found by solving (1.4) using at least 3 values of $T(x)$. The vertical and horizontal derivatives in (1.4) can be evaluated by standard space or -frequency domain methods. Solving (1.4) in this way is unreliable due to the effect of adjacent sources and because the higher values of $N$ are best for depth determination (sources approach monopolarity). Thompson introduces a bias term B.to take care of néarby anomalies and regional effects and solves for $x_{0}, z_{0}$, and $B$ using prescribed values of $N$. Although 3 points could be used, 7 are adopted to provide an overconstrained system of equations and increased statistical reliability. The least 
squares solution of such a system also gives covariance information concerning parameter estimates which are useful for specifying an acceptance criterion for the procedure when automated. Results are plotted in a similar manner to Werner deconvolution with different symbols for the various values of N. Similarly, the pattern of the depthestimates may be diagnostic of certain geological features.

\subsection{Continuous methods}

\subsubsection{Peters's method}

Peters (1949) proposed that a combination of downward continuation and the equivalent stratum principle for magnetic fields could be effective in mapping the topography of an interface separating uniformly but differently magnetized media. The basis of Peters.'s appoach is the use. of the approximate linear relation between observed field and interface relief in the following manner.

The potential at a point $\left(x_{0}, y_{0}, 0\right)$ produced by the relief $h(x, y)$ (measured relative to the average depth $d$ of the. interface) is

$$
A\left(x_{0}, y_{0}, 0\right)^{\prime}=J \cdot \frac{\partial}{\partial \vec{\beta}} \int_{-\infty}^{\infty} d x d y \int_{d}^{d+h(x, y)} \frac{d z}{\left(\left(x_{0}-x\right)^{2}+\left(y_{0}-y\right)^{2}+\left(z_{0}-z_{1}\right)^{2}\right)^{1 / 2}}
$$

where $J$, and $\vec{\beta}$ are the magnitude and direction of the 
- magnetization; respectively. When $|h(x, y)|<<$ the following approximate relationship holds:

$$
A\left(x_{0}, y_{0}, 0\right)=J-\frac{\partial}{\partial \vec{\beta}} \int_{-\infty}^{\infty} d x d y \int_{d}^{d+h(x, y)} \frac{h(x, y) d z}{\left(\left(x_{0}-x\right)^{2}+\left(y_{0}-y\right)^{2}+\left(z_{0}-z\right)^{2}\right)^{1 / 2}}
$$

The use of the continuation formula between the planes $z=0$ and $z=d$ results in the expression of potential in terms of the topography:

$$
A(x, y, d)=2 \pi J h(x, y)
$$

If total field measurements are used, these are easily integrated to give the potential which can be continued downward and converted into relief once $d$ and $J$ are specified. When the condition $|h(x, y)|<d$ is well satisfied, the first linear approximate topography (FLAT) given by (1.6) provides an acceptable solution to the problem. This approximation forms the basis of many approaches to the topography inversion (TI) problem. Peters originally carried out the above operations using a set of coefficients applied to the data in the spatial domain. This is now more efficiently carried out using Fast Fourier Transform (FFT) methods (e.g. Kanasewich, 1980, Chap. 3.)

A direct descendent of the Peters method has been developed by Baldi and Unguendoli (1978; 1981) who express the unknown topography $h(x)$ lonly profile data is 
considered) as a polynomial. The coefficients are then determined by a least squares approach, the disadvantage lying in the necessity for a priori specification of the degree of the polynomial.

\subsubsection{Harmonic analysis method}

Hahn (1966) developed a method based on Nagata's(1938) expressions for the total magnetic field produced by a sinusoidal magnetization distribution in a horizontal plate. The basis of the method is a. first order approximation through which Hahn converts magnetization into basement relief. The magnetization

$$
J=J \underset{u, v}{ } \cos ((u+v)-\varphi)
$$

in an infinite half-space with top surface $z$, produces the field

$$
T(z)=2 \pi J \underset{u, v}{ } D \cos ((u+v)-\varphi+\pi-2 I)
$$

where

$$
\begin{aligned}
J_{u, v} & =\text { amplitude coefficient of magnetization } \\
u_{,} v & =\text { wavenumbers in } x, y \text { directions } \\
D & =\text { geomagnetic field direction factor } \\
\varphi & =\text { phase angle of } u, v t h \text { magnetization component } \\
I & =\text { inclination of geomagnetic field, }
\end{aligned}
$$

- Evidently a phase shift occurs between the magnetization and its field. The amplitude of (1.9) is then 


$$
\left|T(z)_{h s p}\right|=2 \pi J \underset{u, v}{D} \text {. }
$$

Hahn expands the observed field into a Fourier series then continues each component down to an estimated average depth z. The basement is assumed to have a constant magnetization which must be estimated independently. Considering the field produced at a height $z$ by a magnetized plate whose upper and lower boundaries are $z$ and $z+\Delta z$ (the $z$-axis points downward), and that produced by a half-space similarly magnetized and with a coincident upper surface, one can write

$$
|T(z)|_{p \text { ate }}|=| T(z) \operatorname{hsp} \mid\left(I-e^{-V\left(u^{2}+v^{2}\right) \Delta z}\right)
$$

If the thickness $\Delta z$ is small compared to the length $\dot{V}\left(u^{2}+v^{2}\right)$, the horizontal plate of variable magnetization and constant thickness produces the same field as a plate with constant magnetization and variable thickness. This is apparent if the exponential term in (1.11) is expanded in a Taylor's series and only first order terms are retained. Then

$$
\left|T(z)_{\text {plate }}\right|=\left|T(z)_{h s p}\right| V\left(u^{2}+v^{2}\right) \Delta z
$$

and substituting in (3) gives

$$
\mathrm{J}_{\mathrm{J}, \mathrm{v}} \Delta \mathrm{z}_{\iota}=\frac{\left|\mathrm{T}(\mathrm{z})_{\text {plate }}\right|}{V\left(\mathrm{u}^{2}+\mathrm{v}^{2}\right) 2 \pi \mathrm{D}}
$$


Equation (1.13) shows the interrelationship of $\mathrm{J}_{u, v}$ and $\Delta z$. A linear relationship exists between the total field and the relief as long as the latter is small. The relief corresponding to the observations can be constructed by successively continiling frequency components (called partial waves by Hahn(1966)) down to the specified depth. Examples of harmonic analysis have been given by Hahn et al(1976). and Mishra(1984).

\section{2 .2 .3 Parker-oldenburg method}

Rearranging Parker's(1973) formula for the rapid calculation of the potential field arising from a single interface, Oldenburg (1974) derives an iterative scheme for the solution of problem TI. As noted by him, the speed of the forward algorithm enables the approach to be practical. Oldenburg considers the gravity case for which the Fourier transform of the gravity anomaly $g(x)$ is given by (Parker, 1973)

$$
\mathscr{F}[g(x)]=2 \pi G \rho e^{-|\vec{k}| z_{0}} \sum_{n=1}^{\infty}\left|\frac{\vec{k}}{n !}\right|^{n-1} \mathcal{F}\left[h^{n}(x)\right]
$$

where $G, p$ and $z_{0}$ are the gravitational constant, density contrast and average depth of the interface. The derivation of (1.14) is outlined in section (2.6.2). Separating out the first term in the series gives 


$$
\begin{aligned}
& r
\end{aligned}
$$

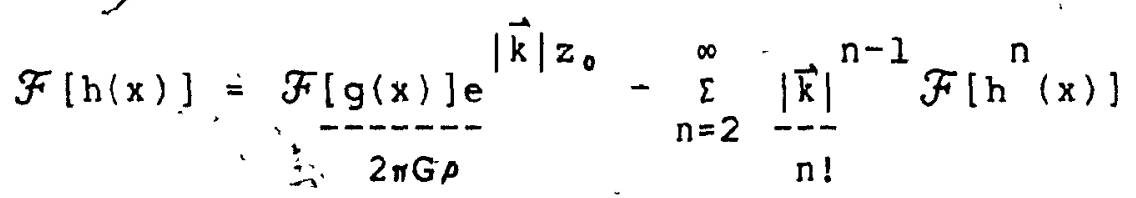

Equation (1.15) is in the form of a successive approximations formula which can be used iteratively (starting with $h(x)=0$ ) to determine the interface topography $h(x)$. The latest approximation to $h(x)$ is substituted into the RHS of $(1.15)$ and a new $h(x)$ calculated on the LHS; this. procedure is continued until one or more convergence criteria are met. As noted by oldenburg, using (1.15) in its rudimentary form usually leads to divergence of the algorithm due to the exponential factor magnifying the high wavenumber content of the data. Remedial action takes the form of low-pass filtering the RHS of (1.15) at each iteration.

The inversion thus involves three free parameters: $\rho, z_{0}$ and the form of the filter, most importantly, its cut-off frequency. Oldenburg investigates the role of filtering on the resulting topographies, noting that a filter can usually be found that guarantees convergence, although whether it produces an estimate of the topography that satisfies the observed data is unpredictable:

Sprenke and Kanasewich (1982) have also used the Parker algorithm for computing isostatic anomalies over large areas. They then use the Parker-oldenburg inverse method to solve for density distributions in the upper crust and upper 
mantle, and the topographies of the ,Riel and Mohorovicic discontinuities. Additional seismic control is used to reduce the nonuniqueness of the solutions.

Goldflam et al (1977) report on another frequency domain approach to problem TI. Using generalized Fourier transforms they derive expressions for the gravity field arising from a simple sinusoidal interface. A method for the inverse problem is then presented but seems to be rather impractical due to the limitations of the chosen model.

\subsubsection{Continuation method}

Courtillot et al (1974) show how the inverse problem for a source distribution consisting of an irregular layer with relief $G$, magnetization $J$, and horizontal base $(z=0)$, can be formulated as a continuation problem. This arises from an approximation of the potential, $V(x, H)$ due to the above source which is given by

$$
V(x, H)=-2 \pi J \int_{-\infty}^{\infty} \frac{H}{\left(x-x_{0}\right)^{2}+H^{2}} G\left(x_{0}\right) d x
$$

The solution for $G\left(x_{a}\right)$ gives the FLAT (section 1.2.2.1). Inspection of (1.16) shows it, is, to within a constant factor, in the form of a general continuation formula for two-dimensional fields. The good quality of the approximation leads to $(1.16)$ being the first step in a 
rapidly convergent iterative scheme, where at each step a linear integral equation of the form (1.16) is solved. Despite the relative ease of solving such equations, the kernels depend on the most recent approximation to the topography $G_{n}\left(x_{0}\right)$ computed from the (n-I)st step. They have the general form

$$
\frac{Y_{n-1}\left(x_{0}\right)-H}{r_{n-1}^{2}\left(x_{0}\right)}
$$

where $r_{n-1}^{2}=\left(x-x_{0}\right)^{2}+\left(G_{n-1}\left(x_{0}\right)-H\right)^{2}$. So "the initial advantage of the simple form of (1.16) is lost and numerical integration is needed for solving the resulting set of equations. This limits the method to small data sets.

Courtillot et al studied the convergence properties of the method empirically and outlined ways of determining an upper bound on $H$ and a lower bound on $J$ when these parameters are poorly known. They also show that a hyperbolic-like limit exists separating regions of convergence and divergence of the algorithm as $H$ and $J$ are varied. With minor modifications, the distribution of magnetization in a geometrically specified source region can also be found in the above manner.

1.2.2.5 Gerard and Debeglia

Gerard and Debeglia (1975) proposed an integrated automatic procedure for determining average depth and 
magnetization of ap interface, and its topography. The interface is modelled by placing infinite depth prisms of dimensions equal to the data spacing and whose centres coincide with data points. Basement topography is expressed as an average depth plus a local variation with an associated probability density function $p(x)$. The energy density spectrum of such a model field is dependent on the form of $p(x)$ so Gerard and Debeglia choose simple depth distributions and find the best match to the observations. Once this is done, this effect is removed and the slope of the spectrum used for the average depth'( $z$ ) determination.

The magnetization/density contrast is then found based on an argument concerning the conservation of mass above the average depth and parameters determined from the statistical analysis. With the $(Z, J)$ pair found, an iterative technique is used for topography calculations. This takes the form of an approximate downward continuation and conversion into topography in the spatial domain using the equivalent stratum principle (section (1.2.2.1)). Successful methods to this end have also been outlined by Bott(1960) and Quereshi and Mula(1971). Gerard and Debeglia use a first order approximation for the continuation:

$$
\begin{aligned}
& G_{z}=G_{0}+z T_{0} \\
& z={ }^{*} / 2 \pi J
\end{aligned}
$$


where $G_{z}$ and' $G_{0}$ are gravity values at depth $z$ and on the observation plane, respectively, $T_{0}$ is the magnetic field (pole reduced) and $J$ is the magnetization contrast. Similar expressions result for magnetic data. $z$ is initially given by the spectral analysis and is constant. Subsequently'z is variable and the $G_{0}$ and $T_{0}$ are replaced by their corresponding residual quantities during the iterative phase of the algorithm. To provide stability for the continuation, data is low-passed before the conversion to depth estimates.

\subsubsection{6 wiener filtering}

Optimum wiener filters have been designed by Gunn (1975;1976) to provide direct mappings of interfaces and layer thicknesses for both magnetic and gravity data. The total magnetic field on the plane $z=0$ arising from an interface can be 'expressed as

$$
T(x, y, 0)=2 \pi M \star D_{1}(1, m, n) \star D_{2}(L, M, N) \star L(x, y) \star H(x, y, h)
$$

where * denotes convolution and

$M=$ magnetization contrast at interface-

$D_{1}=$ geomagnetic field direction factor

$D_{2}=$ magnetization direction factor

$L=$ relief of interface

$H=$ Eactor arising from average depth $h$ of interface

Using this approximate relationship between field $\mathrm{T}$ and relief $L$, filters can be derived to deconvolve the effects 
of $D_{1} ; D_{2}$, and $H$ defined above. The practical application of this approach is limited by the need to specify a desired output during the filter design stage when the potential field generation is considered in terms of linear system theory: a first approximation of the structure to be mapped is needed. When all auxiliary parameters are specified correctly, Gunn (1976) notes that the restriction of the above equation to small reliefs is not rigorous.

\subsubsection{Generalized 1 inear inverse approaches}

Pedersen (1977) solves the topography inversion problem for profile data using generalized matrix-inversion. The relief function is specified by a number of $(x, z)$ points such that it has a step-like character. Values for the $x$ coordinates are governed by the measurement points and need not be equally spaced. The $z$ coordinates are defined with respect to an average depth which, along with the magnetization of the model is kept fixed during iterations." Pedersen derives analytical expressions for the partial derivatives of the data with respect to the model parameters and notes that the magnetic derivatives decay faster than the corresponding gravity problem, implying that the former is less linear and requires a better starting model. Pedersen's approach is basically the usual inverse procedure described in detail in section (2.5.2). The method seemís to work well although the convergence of the iterative 
procedure is highly.sensitive to the starting model; Pedersen's initial guesses differ little in character from the final solutions. Also the final model consists of number of infinite depth.prisms giving a rather rough topography which should be smoothed for a more realistic result.

Simpler least squares matrix methods for subsurface relief determination have been discussed by Tanner (1967), Kunuratnam (19732) and Dyrelius and Vogel (1972).

\section{$\underline{1} \cdot \underline{3}$ Discús,sion}

Within the group of continuous methods described, a distinct division can be made based on the accuracy of the Einal solution. The methods of Peters (1.2.2.1), Hahn $(1.2 .2 .2)$ and Gunn $(1.2 .2 .6)$ all produce the FLAT as their final solution. These are essentialrly lineár methods, implicitly involving'a downward continuation of the observed field and conversion into relief. As such, they are non-iterative in nature and do. not consider the discrepancy between the observed and calculated fields. Assuming all other parameters have been chosen correctly, the misfit is dependent on the character of filtering employed in the continuation (see section (3.2.1)). Although originally developed in the space domain, Peters's and Gunn's approach can be transformed to the wavenumber domain with a resulting decrease in computational cost due to the rapidity of

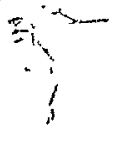


available Fast Fourier Transform (FFT) algorithms. These three methods can be classed as computationaliy cheap.

- The remaining methods are iterative, with the ".: "

Parker-oldenberg (PO) and courtillot approaches both providing the FLAT, on the first iteration. The cost of the evaluation of the partial derivatives at each iteration dictates whether a particular method is viable for use on large data sets. Derivative evaluation and the subsequent solution of a set of linear equations (in order to determine parameter correctionst becomes prohibitive for the courtillot and general linear inverse (GLI) approaches . although the accuracy of these solutions can be expected to be superior, especially in highly nonlinear situations. The PO and Gerard and Debeglia (GD) methods use no derivative information, the $\mathrm{GD}^{\text {ra }}$ approach employing only a first order approximation for downward continuation; in both cases the cost is low. A trade-off is apparent between cheapness and accuracy. It is interesting to note that all meithods of section (1.2.2) must incorporate some damping, the form of which is most important to achieving an acceptable solution; only in the $\mathrm{PO}^{\mathrm{GD}}$ and $\mathrm{GLI}$ methods is this considered. In the former two cases arbitrary filtering based on empirical tests is used while for the GLI approach" the problem eigenvalues determine the type of filtering to be used. The latter case is the most objective as' filter parameters are not specified prior'to data inversion. 
27

1. 4 Aims of thesis

The primary purpose of this thesis is the development of a cost-effective yet reasonably accurate interpretational tool appropriate for use on large gridded potential field data sets. Particular properties to be incorporated in such a system are:

1. Low cost of construction of a solution and calculating model effects.

2. Minimal computer storage requirements.

3. Stability of model parameters in the presence of "noise" in the data.

4. Versatile interpretive model.

5. Non-uniqueness of parameter estimates easily investigated.

6. Minimum of"abitrariness at all stages of computing a solution. 
CHAPTER $2 \underline{2}$

\section{$\underline{2} \cdot \underline{\text { Introduction }}$}

The problem of determining the topography of an interface from potential field data can be considered as an inverse problem in geophysical data interpretation, In inverse problems the aim is to estimate a set of model parameters which describe a physical system such that the data calculated from the model are in agreement with the observed data to within a prescribed degree of error. Obviously before attempting the estimation, the forward problem of obtaining calculated data from a set of model parameters must be solved.

The inverse problem can be divided into two parts: solution construction and. solution appraisal. For the former, the least squares estimation procedure is appropriate; it has found widespread use due to the simplicity of the resulting equations, the minimum statistical information required concerning problem variables, and conceptual ease in its implementation. Importantly, the addition of a priori information is accommodated in a straightforward manner. However, numerical 
difficulties arise when the problem is ill-posed and small changes in the data cause large variations in the resulting estimates. In such cases the situation is not improved by more accurate data, therefore some form of constraint (a priori information) must be introduced into the solution construction to stabilize model estimates. Linear inverse theory (Backus and Gilbert, 1967;1968;1970) provides a powerful tool to this end.

Based on thi's theory, the appraisal of a given solution (no matter how derived) can be carried out, evaluating such measure's as the resolving power of the data, their information content, and accuracy of the solution. With this information, the fundamental question of solution uniqueness can be addressed in a quantitative manner. All geophysical problems are plagued with non-uniqueness in one sense or another. Four separate sources of non-uniqueness can be distinguished: (i) observed data is finite and usually discrete, whereas the solution is a continuous function, (ii) the data are noisy, (iii) the actual physical system modelled is represented by a non-unique and incomplete set of parameters, (iv) fundamental theoretical ambiguity e.g. Gauss's theorem in potential field theory (5) states that an infinite number of density distributions can produce the same gravity anomaly.

In this chapter the determination of a single interface topography is approached using generalized inverse theory. 
This is extended to the more general $n$-layered case for both magnetic and gravity data. The inclusion of linear equality constraints is also outlined.

\section{2 Linear least-squares inversion}

Although most geophysical problems are nonlinear, the method of solution is usually based on linear theory. When observations are a linear combination of model parameters, their relation can be written in the form of a linear convolutional equation (for the continuous case)

$$
y(s)=\int_{-\infty}^{\infty} k(s-t) x(t) d t
$$

where the $y$ are data, $x$ are parameters and $K$ is a kernel independent of $s$. Equation $(2.2 .1)$ is a Fredholm integral equation of the first kind and in matrix (discrete) form becomes

$$
y=A x
$$

with $y$ an n-vector, $x$ an m-vector and $A$ an nxm matrix whose ijth element is given by $k(j-i)$. It should be noted that the * conversion of $(2.2 .1)$ to $(2.2 .2)$ implies the application of an appropriate quadrature formula for the discretization. Equation (2.2.2) then represents a mapping of $\mathcal{R}_{m}$ onto $\boldsymbol{R}_{n}$ by 
the operator $A$.

If the measurements were perfect and $x$ was sufficient to parameterize the actual model, (2.2.2) could be salved exactly. However, the observations are noisy and (2.2.2) only approximates reality. The solution of (2.2.2) results in some discrepancy (the residuals e) between the measurements $y$ and the calculated data $\hat{y}$.

$$
\text { e }=y-\hat{y}
$$

. In order that the solution of $(2: 2.2)$ is optimized, the size of the residual vector e should be minimized with respect to some norm. Assuming a linear model the solution can be expanded in a Taylor's series about an initial estimate $x^{0}$; only the first series term is needed due to the linearity:

$$
\hat{y}=\hat{y}^{0}+\left.\frac{\partial y_{i}}{\partial x_{j}}\right|_{x=x^{0}}\left(x-x^{0}\right)
$$

The caret denotes the calculated data of the corresponding model. Equation $(2.2: 4)$ becomes in matrix terms

$$
\hat{y}=\hat{y}^{0}+A_{i} \Delta x^{\prime}
$$

with $\Delta x$ representing the perturbation to the model and $A$ is the nxm Jacobian matrix of partial derivatives with the ijth element given by $\partial y_{1} / \partial x_{j}$. If $d$ is the discrepancy between data and model response, from (2.2.3) and (2.2.5),

$$
e=d-A \Delta x
$$


Choosing to minimize the residual vector e using the $1-2$ norm leads to minimizing the quadratic form $s=e^{t} e$. Setting $\mathrm{dS} / \mathrm{d} \Delta \mathrm{x}$ to zero gives

$$
\frac{d}{d \Delta x}\left(\Delta x^{t} A^{t} A \Delta x-d^{t} A \Delta x-\Delta x^{t} A^{t} d+d^{t} d\right)=0
$$

resulting in the normal equations of ordinary least squares:

$$
A^{t} A \Delta x=A^{t} d
$$

with the solution for the parameter change being

$$
\Delta x=\left(A^{t} A\right)^{-1}{ }^{A} \mathrm{t} d
$$

$\Delta \mathrm{x}$ given by (2.20) results from a straightforward minimization of the sum of squares, $e^{t} e$, and involves no statistical, assumptions whatsoever (Beck and Arnold, 1977, p.135). The resulting estimator is unbiased and in the presence of uncorrelated and constant variance measurement error provides the minimum variance solution of (2.2.2) (Beck and Arnold, 1977, P.239). Matrix A $A^{k}$ is of dimensions $\mathrm{mxm}$, so for the solution to exist its rank must be $m$, implying that all eigenvalues are non-zero.

In many problems $A^{t} A$ is singular due to zero eigenvalues or eigenvalues that are computationally indistinguishable from zero. In these cases the least squares inverse (equation (2.2.9)) contains excessively large elements 
resulting in unstable estimates for $\Delta x$. One possible remedy is to increase the magnitude of the small eigenvalues or, alternatively, to remove them from the solution. Increasing the eigenvalues can be effected by the inclusion of a priori information into the problem. This usually takes the form of data and parameter covariances, where it is normally assumed that these are independent. The resulting function to be minimized is then

$$
Q=(d-A \Delta x)^{t} D^{-1}(d-A \Delta x)+\Delta x^{t} P^{-1} \Delta x
$$

The data covariance matrix $D$ takes into account any differences in type and accuracy of observations. Similarly, the parameter covariance $P^{\prime}$ discriminates against poorly determined parameters. Differentiating (2.2.10) 'with respect to $\Delta x$ leads to the solution

$$
\Delta x=\left(A^{t} D^{-1} A+P^{-1}\right)^{-1} A^{t} D^{-1} d
$$

The estimator in $(2.2 .11)$ is commonly known as the stochastic inverse, and was derived for the underdetermined case by Franklin (1970) under the assumptions that the parameters are the realization of a second order Gaussian process. This assumption is obviated in Jackson's (1979) derivation of $(2.2 .11)$.

From a numerical point of view, the stabilization of the inversion is shown by the addition of $\mathrm{P}^{-1}$, which being 
positive definite (under the assumption of no perfect parameter correlations and no null bariances) ensures the eigenvalues of $\left(A^{t} D^{-1} A+P^{-1}\right)$ are greater than zero. The region of acceptable models then becomes bounded. The a priori constraints need not be of a statistical nature: they may arise from simple physical argu ments. Also, it should be remembered that there are usually some hidden a priori constraints imbedded in a problem e.g. the discretization of data imposes a high frequency limit on the sampled observations.

If no constraints are imposed on parameters, (2.2.11) reduces to

$$
\Delta x=\left(A^{t} D^{-1} A\right)^{-1} A^{-1} d
$$

which is the maximum likelihood solution of $(2.2 .2)$. If $A^{k} A$ is non-singular and the problem is well-posed, $A^{t} D^{-1} A$ is also non-singular, however when $A^{t} A$ is singular, the incorporation of data covariances does not necessarily lead to a well-posed problem (Beck and Arnold, 1977, App. A). In the case of small $\mathrm{m}$, a large data set may suffice to ensure the resulting inverse matrices exist (Jackson, 1979).

Whatever the source of the a priori information, they should be looked upon as 'virtual' measurements (Rodgers, 1976; Jackson, 1979) that provide similar information as the data, and when combined, with the 'true' data determine whether the problem is well-posed or not. 


\section{$\underline{2} \cdot \underline{3}$ Nonlinearity}

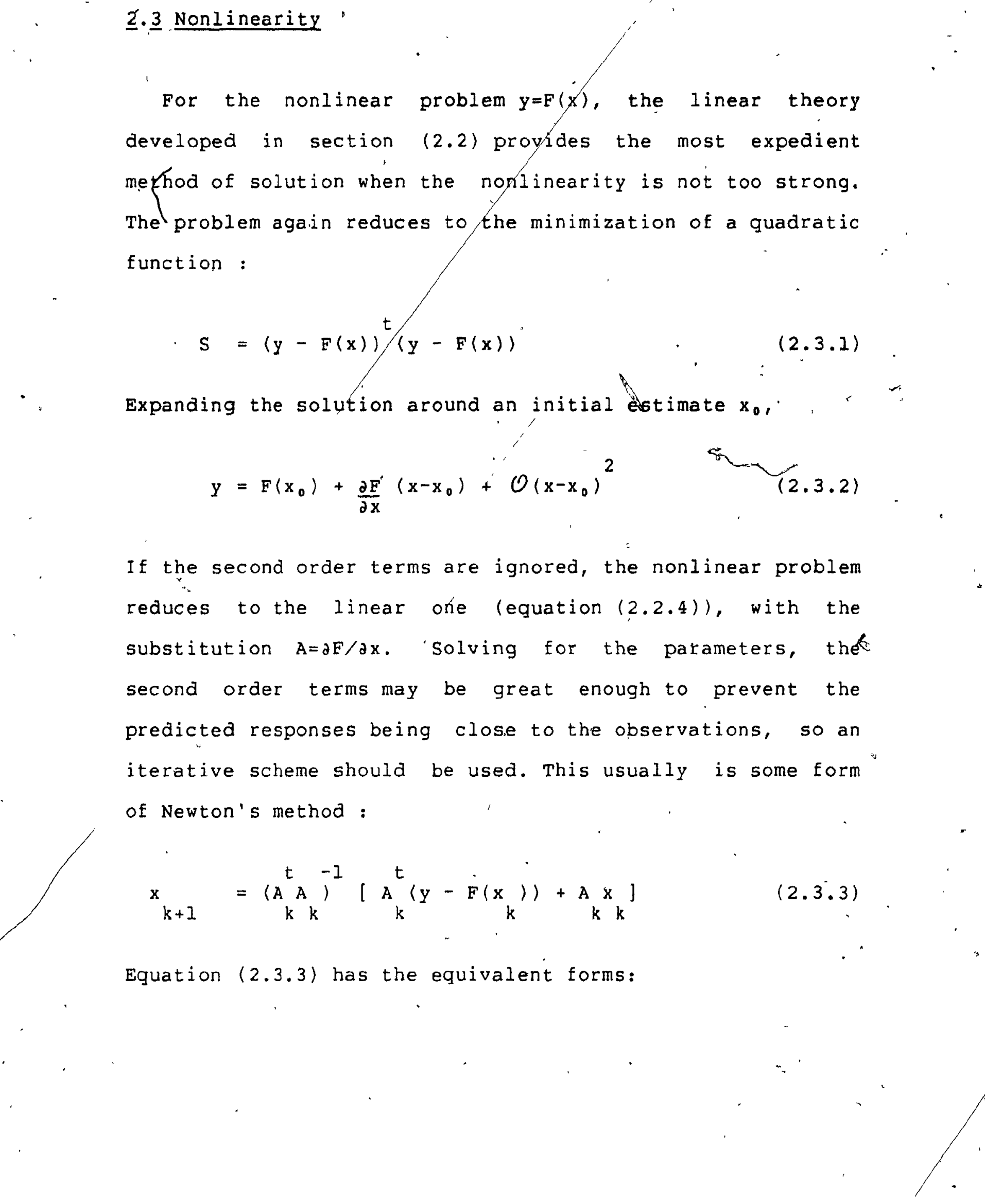




$$
x_{k+1}=x_{0}+\left(A_{k}^{t}\right)_{k}^{-I}{ }^{t}\left[\left(y-F\left(x_{k}\right)\right)-A_{k}\left(x_{0}-x_{k}\right)\right]
$$

and

$$
x_{k+1}=x_{k}+\left(A_{k}^{t}\right)_{k}^{-1} A_{k}^{t}\left(y-F\left(x_{k}\right)\right)
$$

where the latter is most commonly used. Subscript $k$ denotes the kth iteration. The Frechet derivative $\partial F / \partial x$ must be evaluated at each iteration. This can be done analytically or numerically. The most simple numerical scheme (forward differencing) involves two function ( $F(x)$ ) evaluations, hence the magnitude of the problem will determine the most economical approach. Additionally, (2.3.4) requires the inversion of the mxm matrix $A^{t} A$ or the solution of a set of simultaneous equations for every $k$. For extremely large problems, A may be too large to recalculate and $A^{t} A$ too large to invert at each iteration. Matrix updating routines (variable metric methods) are useful in this respect as they involve the approximation of an inverse $\left(A_{k+1}^{t} A_{k+1}\right)^{-1}$ determined directly from information supplied by the previous iteration (Spedicato, 1980).

The convergence of $(2.3 .4)$ is not guaranteed. Even if a solution does exist, the initial guess may be so far away that linearization fails and divergence results. Methods for improving the behaviour of the iterative process are discussed in the next section (2.4). If a single minimum of $S(2.3 .1)$ exists and the nonlinearity is weak, by analogy 
with. Newton's method (e.g. Conte and deBoor, 1.980, p.223), the process $(2.3 .5)$ tends to this minimum with second order convergence (the solution would be reached in one iteration in the linear case). Once an acceptable solution is determined, $=$ a posteriori analysis can be effected using the linearized equations.

For strongly nonlinear problems, random search techniques such as Monte Carlo search should be used. This alternative is attractive when $\mathbf{S}$ is complicated by local minima, saddle points etc. due to, for example, data errors and poor, parameterization. The question of whether a minimum obtained is a global one then arises; this cannot be answered prior to deriving a solution and can only be addressed by carrying out a more exhaustive search. One important advantage of this type of modelling is that models widely separated in parameter space are considered, including those that might not be expected intuitively. Unfortunately, the computational undertaking may be enormous, leading to some form of initial limiting procedure to bound the possible search space.

\section{$\underline{2} . \underline{4}$ Marquardt-Levenberg method}

As alluded to in section (2.2), the presence of small or. zero eigenvalues of $A^{t} A$ causes instability in the solution of the normal equations $(2.2 .8)$. For nonlinear least 
squares, this results in parameter corrections that can be so far from the solution that the linear approximation breaks down and further iterations diverge.

In section (2.2) it was seen that the incorporation of a priori data led to bounded parameters. An approach similar to this was introduced by Levenberg(1944) and further Fefined by Marquardt(1963) to stabilise the iterative nonlinear least squares procedure. Hoerl and Kennard(1970) use the term 'ridge regression' for what is simply the addition of a constant term to the diagonal elements of $A^{t} A$. This gives the solution: is

$$
x=\left(A^{t} A+\theta I\right)^{-1} A^{t} d
$$

$\theta$ is commomnly called the Marquardt parameter. It is easy to show that this addition to the diagonal increases all the eigenvalues of $A^{t} A$ by an amount $\theta$, thus removing instabilities caused by zero and near-zero eigenvalues. The ridge regression root mean square (RMS) error is greater than the standard least squares RMS error in the linear case, but not necessarify so in the nonlinear case (Inmán, 1975). The optimum value of $\theta$ cannot be determined prior to inversion, therefore a valiue of $\theta$ is selected, the RMS error computed, and compared with the previous iteration RMS. If" the error is decreasing, the step is acceptable. When the RMS value increases, $\theta$ is increased (producing smaller parameter corrections) until a decrease in error is obtained 
(e.g. Ku, 1976). Increasing $\theta$ is more likely to produce convergence because as $\theta \rightarrow \infty$, the procedure "reduces to the -method of steepest descent for which convergence is assured. As $\theta-0 ;$ the usual Gauss Newton equations result. specifically, when $\theta$ is much larger than the largest eigenvalue of $A$, then $(2.4 .1)$ simplifies to

$$
\dot{x}=\frac{1}{\theta}{ }^{t} y
$$

where $1 / \theta$ acts as a stepsize parameter primarily influencing the rate of convergence. Although (2.4.2) involves no matrix inversion, it is infamous for producing slow convergence towards a solution. Equation (2.2.9) and (2.4.2) are extreme cases of the general algorithm

$$
x=G A^{t} y
$$

with the choice $G=\left(A^{t} A\right)^{-1}$ giving the ordinary least squares. solution and $G=1 / \theta$ giving the gradient Solution. The value of $\theta$ interpolates between these extremes with the optimum choice producing the best compromise between the simplicity of the resulting equations and the number of iterations required for convergence to an acceptable solution. "The Marquardt-Levenberg method combines in an efficient fashion, rapid convergence with steepest descent at points far from a minimum and with the Gauss-Newton approach in the vicinity of a minimum. 
By analogy with (2.2.11), equation (2.4:1) results from the minimization of the sum of squares of the residuals subject to smoothing or bounding the parameter change, that is, $x^{t} x^{\prime}=c$ (a constant). The function to be minimized is then

$$
s(\underline{c})=e^{t} e^{t}+\left(x^{t} x-c\right)^{t}
$$

where $\theta^{\prime}$ is a Lagrange multiplier. Differentiating with respect to $x$ gives $(2.4 .1)$. It is seen that the addition of $\theta$ is equivalent to the inclusion of a priori information about parameter behaviour.

The Marquardt parameter has an analogue in the construction of wienef filters, wherein constraining filter energy leads to the addition of a constant value to the diagonal elements of the signal autocorrelation matrix. This prewhitening of the input data, before filtering or deconvolution is necessary when the system transfer function approaches zero in the frequency band of interest (clayton and wiggins, 1976).

The statistical interpretation of prewhitening is manifest by forming the stochastic inverse, (equation (2.2.11)) for the case of statistically independent data and parmeters: $P$ and $D$ become or ${ }_{P}^{2}$ and $\sigma_{d}^{2} I$, respectively, with $I$, the identity matrix, and $\sigma^{2}$, variance. The estimator of (2.2.11) becomes 

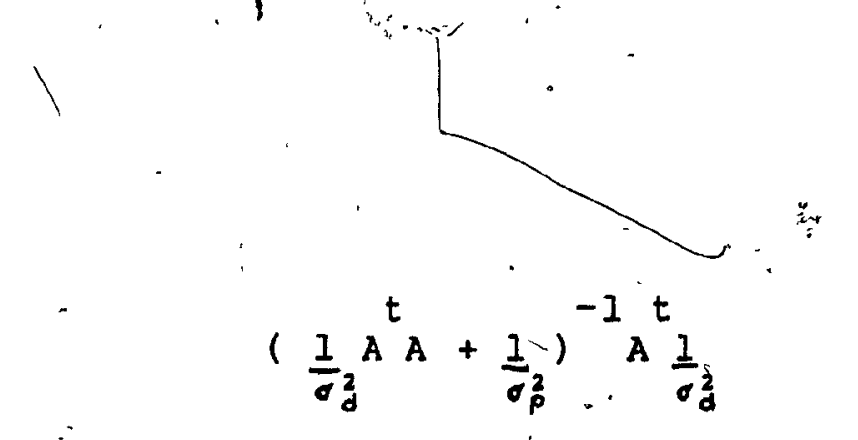

which gives

$$
\left(A^{t} A+c^{2} I\right)^{-I \cdot t}
$$

with $c^{2}=\sigma_{d}^{2} / \sigma_{p}^{2}$; the ratio of datia to parameter variance or the noise to signal ratio. The equivalence of $\theta$ and $c^{2}$ is apparent.

\section{$\underline{2} \cdot \underline{5}$ Inverse theory}

\subsubsection{Backus-Gilbert theory}

Inverse theory can be applied to any problem for which the forward solution is known and a reasonable estimate of the model parameters is available. The general nonlinear problem can be written in linearized form as a Fredholm integral equation of the "first kind,

$$
y_{i}=\int_{a}^{b} G_{i}(r) m(r) d r \quad(i=1, n)
$$

where the $y_{i}$ are data discrepancies (differences between observations and "model responses), $m(r)$ are model perturbations and the $G_{i}(r) / a r e$ Frechet derivatives. The. general solution of $(2.5 .1 .1)$ is $\rightarrow$.

$$
m(r)=\frac{m}{p} \cdot(r)+m_{\perp}(r)
$$


where $m_{p}(r)$ is a particular solution and $m_{\perp}(r)$ satisfies

$$
0=\int_{a}^{b} G_{i}(r) m_{\perp}(r) d r \quad\langle i=1, n\rangle
$$

- $m_{\perp}(r)$ will exist for any finite data set because (2.5.1.1) defines a grossly underdetermined problem. Backus and Gilbert(1967,1968,1970) developed a formalism that deals with the non-uniqueness of the inverse problem in a quantitative manner, and can determine the optimum solution in terms of resolution and variance of parameter estimates. The data in (2.5.I.I) can be regarded as representing $n$ linear combinations or moments of the model. Similarly, the solution. is constructed from linear combinations of the kernels $G(r)$,

$$
\hat{\dot{m}}(r)=\sum_{j=1}^{n} a_{j}\left(r_{0}\right) d_{j}=\int_{a}^{b} m(r) A\left(r, r_{0}\right) d r
$$

From $(2.5 .1 .1)$ and $(2.5 .1 .4)$,

$$
A\left(r, r_{0}\right)=\sum_{j=1}^{n} a_{j}\left(r_{0}\right) G_{j}(r)
$$

$A\left(r, r_{0}\right)$ is the averaging function or resolving kernel and the $a_{j}\left(r_{0}\right)$ are constants to be determined subject to specified constraints. Each $A\left(r, r_{0}\right)$ is essentially a weighting function that controls the detail with which the - true model can be perceived ( $\sec (2.5 .1 .4))$. As $A\left(r, r_{0}\right)$ is made narrower, more detail of the true $m(r)$ is uncovered. 
Only if $A\left(I, r_{0}\right)-\sigma\left(r, r_{0}\right)$ will the exact solution be uncovered. Since a delta function cannot be constructed from finite data, "the $a\left(r_{0}\right)$ are determined so that $A\left(r, r_{0}\right)$ is as close as possible to $\delta\left(r, r_{0}\right)$. Importantly, Backus and Gilbert (1970) showed that the combinations $\hat{m}\left(r_{0}\right)$ are unique and any. other parameters producing the same model response have this same $\hat{\mathrm{m}}\left(r_{0}\right)$. Thus $\hat{\mathrm{m}}\left(r_{0}\right)$ and the associated $A\left(r, r_{0}\right)$ provide the total amount of 'information that can be gleaned from the data concerning model estimates. A number of different criteria can be used to define the discrepency between the averaging function and some desired loften delta-like) function $h(r)$. The simplest criterion is the Dirichlet or least squares, measure where the $a_{j}$ are determined by minimising

$$
\varphi\left(r_{0}\right)=\int_{a}^{b}\left|A\left(r, r_{0}\right)-h\left(r, r_{0}\right)\right|^{2} d r
$$

If $\dot{h}\left(r, I_{0}\right)=\delta\left(r, r_{0}\right)$, the solution of $(2.5: 1.4)$ subject to (2.5.1.5) gives the ordinary least squares solution for the underdetermined case (Aki and Richards, 1980, p.703).

In order to fully appraise the solution, data errors must be considered. If data errors are Gaussian and independent and have variance $\sigma^{2}$, then from (2.5.1.4), the variance of the parameter estimates at $r_{0}$ are

$$
c\left(r_{0}\right)=\sum_{i, j=1}^{n} a_{i} \operatorname{cov}\left[\sigma^{2}\left(r_{i}\right) \sigma^{2}\left(r_{j}\right)\right] a_{j}
$$


To obtain the best combination of resolution and model error, the quadratic form

$$
\Psi\left(r_{0}\right)=\sin r\left(t_{0}\right)+\cos r \varphi\left(r_{0}\right)
$$

is minmised, where $r$, the trade-off parameter varies from 0 to $90^{\circ}$. When $r=0$, minimising $(2.5 .1 .7)$ produces the best resolved model at the expense of large model errors. Similarly, for $r=90$, noise suppression is emphasised with little regard for resolution. The general behaviour of and . $\varphi$ versus $r$ is given in Figure 2.1. Backus and Gilbert (1970) showed that any other ajs calculated give solutions that plot on the right hand side of this curve. The important feature of Figure 2.1 is that a large decrease in model error can be obtained with a minor decrease in resolution. The optimal solution with respect to $c$ and $\varphi$ is found by minimising the distance from the point $\Psi(\varepsilon, \varphi)$ to the origin; this coincides with the stochastic inverse solution (Aki and Richards, 1980, p.706).

\subsubsection{Singular value decomposition}

Backus-Gilbert theory was developed for the continuous case, emphasising the underdetermined nature of the general' inverse problem. Since most data are in discrete form, the theory has been recast using matrix algebra (Wiggins, 1972; Jackson, 1972). Equation (2.5.1.1) then becomes, for the discrete problem 


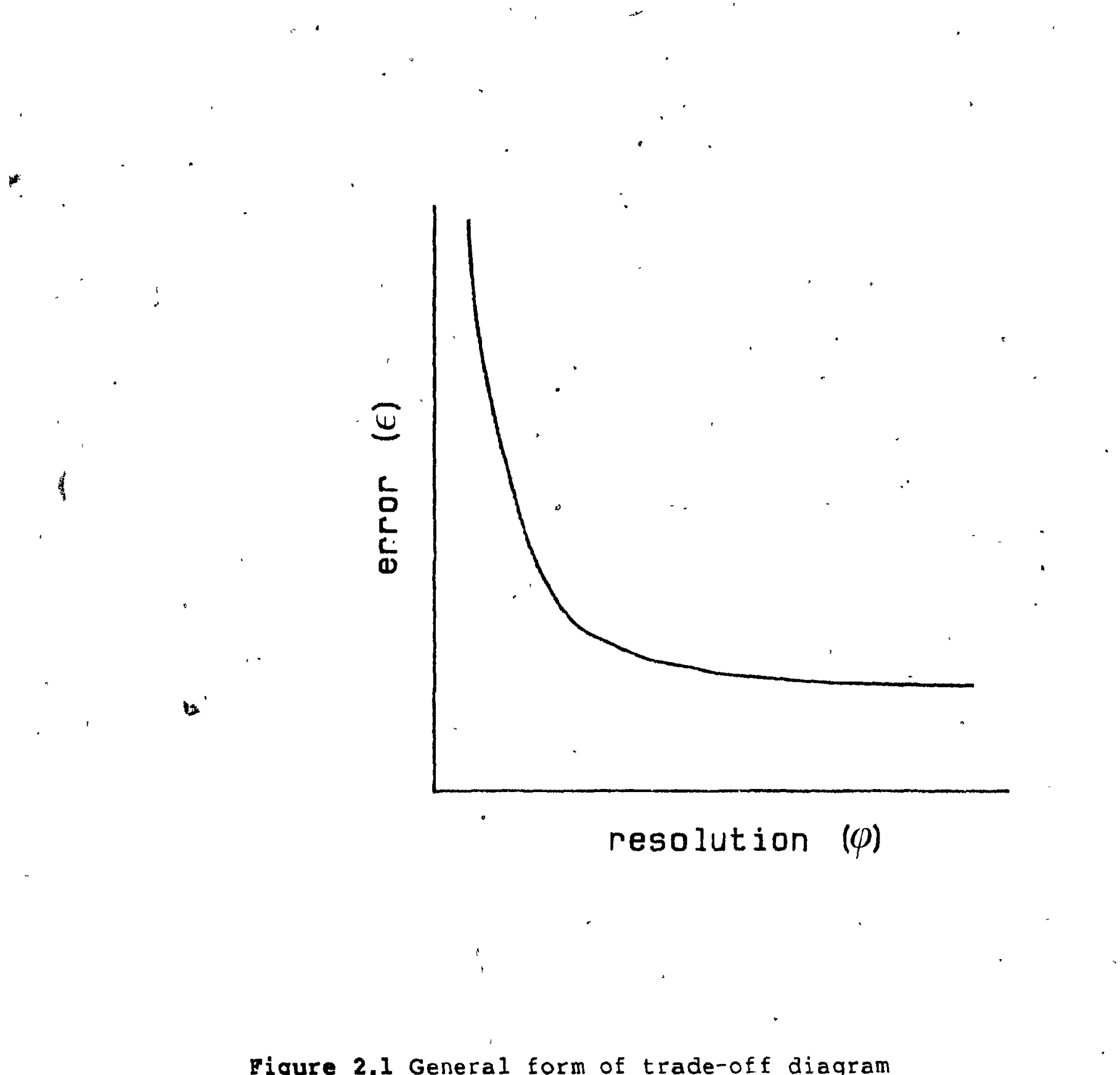




$$
y=A x
$$

The Jacobian $A$ can be factored using the singular. value decomposition (SVD)

$$
A=U \cdot \Lambda \cdot V^{\prime}
$$

(Lanczos, 1963, Ch. 3). The columns of U' span the whole data space and the columns of $V^{\prime}$ span the whole of parameter space. These are eigenvector matrices and are orthonormal. $\Lambda^{\prime}$ is a diagonal matrix containing eigenvalues $\lambda_{i}$, with $\lambda_{i} \geq \lambda_{i+1}$ by convention. When $\operatorname{rank}(A)<\min (m, n)$ and equal to $r$, the matrices in $(2.5 .2 .2)$ can be partitioned into components associated with zero and non-zero eigenvalues:

$$
\begin{aligned}
A & =\left[\begin{array}{ll}
U & U_{0}
\end{array}\right]\left[\begin{array}{ll}
\Lambda & 0 \\
0 & 0
\end{array}\right]\left[\begin{array}{l}
V^{t} \\
V_{0}^{t}
\end{array}\right] \\
& =U \wedge v^{t}
\end{aligned}
$$

where $U$ is $n \times r, A$ is $r x r$ and $V$ is mxr. Equation (2.5.2.3) shows that $A$ can be constructed from only those eigenvectors associated with non-zero eigenvalues. The resulting $U$ and $V$ do not form complete sets and the following properties are evident:

$$
\begin{array}{ll}
U^{t} U=I=V^{t} V ; & V^{t} \neq I ; \quad U^{t} \neq I \\
v_{0}^{t} v=v^{t} v_{0}=0 ; & U_{0}^{t} U=U^{t} U_{0}=0
\end{array}
$$


From $(2.5 .2 .1)$ and $(2.5 .2 .4)$

$$
U_{0}^{t} A x=0
$$

which shows that any model response has no component in $\dot{U}_{0}$. space. Thus if observations contain components in $\mathrm{U}_{0}$ space, there will be a discrepancy between observed and predicted data. Similarly,

$$
A V_{0}=0
$$

implying that any combination of model parameters in $v_{0}$ space does not affect the data. $V_{0}$ space then defines the solution to $(2.5 .1 .3)$ and is the source of non-uniqueness in the problem. The existence of these various spaces depends dịectly on the rank $(r)$ of the problem. If $m>n$, $v_{0}$ space exists ( $U_{0}$ also does if $r<n$ ), if $n>m, U_{0}$ space exists.( $V_{0}$ also does if $r<m)$.

The decomposition (2.5.2.2), provides $a_{i}$ concise representation of the relation between data 'and parameter space and their coupling through the eigenvalues $\lambda$, it also constitutes a ranking of the information contained therein. If a column of $A$ were zero then the corresponding parameter would have no effect on the solution and it can be classed as irrelevant) (Jupp and Vozoff, 1975). If a row of $A$ is zero, the observation is not affected by any model parameter and it provides no information on any parameter. Both of these deficiencies cause zero eigenvalues in $A$, as does 
linear dependence between rows or columns of A. For example, when rows are dependent, some redundant data exist.

Writing $(2.5 .2 .2)$ as a sum of outer products,

$$
A=\sum_{i=1}^{r} \lambda i_{i} i^{t}
$$

It is apparent that the eigenvectors that determine the gross structure of $A$ are those related. to large eigenvalues. Those corresponding to zero eigenvalues are only useful in characterising the non-uniqueness of the solution. Writing (2.5.2.1) with the SVD of A

so

$$
y=U \wedge v^{t} x
$$

or

$$
u^{t} y=\wedge v^{t} x
$$

$$
\beta=\Lambda, \alpha \text {. }
$$

with $U^{t} y=\beta$ and $V^{t} x=\alpha$. The reparameterization to $\alpha$ and $\beta$ shows that those c's related to large eigenvalues are the parameter combinations that have the greatest effect on the observations and hence are the/ best determined by the data. Consequently those combinations with small eigenvalues are poorly determined.

In solving (2.5.2.1) the inverse of $A$ is required. For rectangular matrices, which $A$ usually is, the generalized or natural inverse $H$ (Ĺanczos, 1963) is appropriate and is defined in terms of the SVD of $A$ : 


$$
H=V \wedge^{-1} U^{t}
$$

If $A$ has rank $m, H$ reduces to the least, squares inverse $\left(A^{t} A\right)^{-1} A^{t}$. Similarly when the rank is $n$, th is equivalent to $A^{t}\left(A A^{t}\right)^{-1}$. H has the important property that the solution of $y=A x$ given by

$$
x=H y:=V \wedge U^{-1} U^{t}
$$

minimises the RMS error and the length of the solution vector ||$x^{t} x||$. The inverse $H$ is the Moore-Penrose (Penrose, 1955) inverse of $A$ in that it satisfies all four of the following conditions:

$$
\begin{aligned}
\text { AHA }=A & (A H)^{t}=A H \\
H A H=H & (H A)^{t}=H A
\end{aligned}
$$

The first two conditions are necessary and sufficient for $x=H y$ to be the solution that minimizes the RMS error. The last two ensure that this solution also minimizes $\left\|x^{t} x\right\|$ (Noble, 1976).

Expressing $x$ in series form following (2.5.2.5)

$$
x=\sum_{i=1}^{r} \lambda_{i}^{-1} v \underset{i}{u}{ }_{i}^{t}
$$

which shows the presence of zero eigenvalues causes undue magnification of data values and any noise they contain. For 
this reason the summation in $(2.5 .2 .9)^{\prime}$ stops at $r$; the number of non-zero eigenvalues in A. However, instability still exists due to small eigenvalues and the problem of determining the exact "rank of $A$. This causes $x$ to be large in the directions of the parameter combinations related to the small eigenvalues and the important information supplied by larger eigenvalues is lost. It may then be necessary to limit the sum (2.5.2.9) to a value $r^{\prime}$, smaller than $r$, to bound the estimates. Such a 'sharp cut-off' procedure (Wiggins; 1972) is appropriate for problems whose eigenvalue spectrum shows a natural division between large, relevant eigenvalues and smál, unimportant ones. An alternative procedure is to employ the damping properties of the. Marquardt-Levenberg $(M-L)$ method (section (2.4)). If thedamped solution of $(2.4 .1)$ is written using the SVD of $A$, then

$$
\begin{aligned}
& x=\left\langle v \wedge v^{2}+\theta I\right\rangle^{-1} v \wedge U^{t} y \\
& \forall b=v \wedge U^{t} \cdot y
\end{aligned}
$$

Instead of the usual $\Lambda^{-1}$ matrix containing elements $1 / \lambda_{i}$ in the inverse $H$, the Marquardt-Levenberg method is equivalent to using $\lambda_{i} / \lambda_{1}^{2}+\theta$, thus avoiding division by zero even if $\lambda_{i}-0$. This approach is just one of a class of damped approximate inverses (Jupp and Vozoff, 1975) which differ in 
their treatment of small, non-zero eigenvalues. Marquardt (1970) has pointed out the similarities between $M-L$ inverses and generalized ones and suggests that the former are appropriate for- dealing with small eigenvalues and the latter for problems with zero eigenvalues. This is especially, true for the nonlinear problem since deleting small eigenvalues from the solution at the beginning of the iterations restricts the parameter search prematurely, to a subspace (Dennis, 1977); such eigenvalues may be important in the model space around the true solution.

The discrete equivalent of the Backus-Gilbert measure of resolution, is obtained by substituting (2.5.2.1) into (2.5.2.8) giving,

$$
x=H y=H A x_{r}
$$

with $x_{x}$ being the true solution. The quantity $R=H A$ is the resolution matrix (Jackson, 1972; Wiggins, 1972) which maps the entire. set of solutions into a single vector $x$. The closer $R$ is to the identity matrix $I$, the better the parameters are resolved. The rows (or columns) of $R$ play the same role as the resolving kernels in the continuous case, which can be seen by comparing (2.5.2.11) and (2.5.1.4). Similarly, from (2.5.1.4), the constants a are identical to the rows of the inverse matrix $H$. The Lanczos inverse has the useful property that the resulting resolution matrix approximates I in a least squares sense (Jackson, 1972). 
Using the SVD of $H$ and $A$,

$$
R=H A^{\prime}=V \wedge \wedge U^{-I} \Delta v^{t}=v^{t}
$$

Only if the rank of A is m, will V contain a complete set of eigenvectors, and $R=I$. When $\operatorname{rank}(A)=r<m$, then onily $r$ independent parameters can be determined from the data.

The covariance of the solution estimates, $C$, follows from, $(2.5 .1 .8)$ and is

$$
\mathrm{C}=\sigma^{2} \mathrm{HH}^{\mathrm{t}}
$$

with $\sigma^{2}$ the data variance (assuming statistically" independent, constant variance noise). Using the corresponding SVD's and setting $\sigma^{2}=1$,

$$
C=V \wedge v^{-1} U^{t} \wedge v^{-1}=V \wedge^{-2} v^{t}
$$

Equation (2.5.2.13) shows the deleteriousineffects of small, eigenvalue's on the solution variance. This situation is " improved by removing the small eigenvalues and their associated eigenvectors, which from (2:5.2.12) causes poorer resolution, but from (2.5.2.13) improves the behaviour of the parameter variances. Thus the trade-off between solution variance and resolution is demonstrated as a function of the number of eigenvalues retained in the problem. This carries through to the damped inverse case: increasing damping surpresses the contribution of those parameter and data 
components related to small eigenvalues.

Comparing $(2.4 .2)$ and $(2.5 .1 .9)$ it is apparent that varying the Marquardt (or damping) factor is equivalent to changing the trade-off parameter $r$. In Backus-Gilbert appraisal, $r$ is varied until the optimal combination "yof resolution and stability is found; this should only be undertaken. with the understanding that only one value of $r$ is specified by the a priori information.'

Appraisal in terms of the importance of data is achieved by 'calculating the information density matrix (Wiggins, 19.72) given by $S=A H$. From $(2.5 .2 .1)$ and $(2.5 .2 .8)$,

and

$$
y=A x=A H y
$$

$$
s=U \wedge \cdot v^{t} v \wedge^{-1} U^{t}=U U^{t}
$$

When $r=n, S=I$, otherwise $S$ only approximates the, identity matrix. S provides a measure of the information contained in a data set, redundant, data causing. $\mathbf{S}$ to have, smooth rows, poorly peaked at the diagonal.

\section{$\underline{2} \cdot \underline{6}$ One layer inversion}

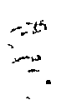

2.6.1 Introduction

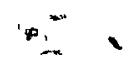

In this section, a method for the rapid inversion of magnetic (or gravity). data in terms of a single interface is developed. The technique is intended primarily for use in the interpretation of large data sets, producing a real $\therefore$ 
physical model that is not constricted, by the usual approach of specifying, source geometries prior to inversion. All sources are modelied simultaneously under the assumption of a continuously magnetised layer. It is shown that linear equality constraints can be incorporated easily, causing a minor increase in the total computation time.

\subsubsection{Forward problem}

In order for the proposed inversion method to be applicable to varying problems, the model used should be as versatile as possible. For this reason the method of rapid potential field calculation of. Parker (1.973) has been adopted. The notion of rapidity is paramount because providing-model responses constitutes an appreciable part of the total computation time. Parket's algorithm results, from Foufier transformation of the potential due to a layer bounded below by the horizontal plane $z=0$ and the surface $z=h(r)$ above. The potential of such a layer at' $\vec{r}_{0}^{\prime}$ is given by

$$
A\left(\vec{r}_{0}\right)=-J(\vec{r}) \int_{D} d S \int_{0}^{h(\vec{r})} d z \vec{j} \cdot \nabla \frac{1}{\left|\vec{r}-\vec{r}_{0}\right|}(2.6 .2 .1)
$$

$J(\vec{r})$ is the magnitude of magnetization at $\vec{r}_{*}$ and $\vec{j}$, its. direction (assumed constant over the area D). Making use of. the Fourier transform pairs 


$$
\begin{aligned}
& \vec{j} \cdot \nabla \cdot \vec{j} \cdot(i \vec{k},|\vec{k}|) \\
& \frac{1}{\left|\vec{r}-\vec{r}_{0}\right|} \Leftrightarrow \frac{2 \pi e^{-i \vec{k} \cdot \vec{r}-|\vec{k}|\left(z_{0}-z\right)}}{|\vec{k}|} \Leftrightarrow
\end{aligned}
$$

(where $\vec{k}$ is wavenumber and $z_{0}$ is the height of the observation plane) and taking the Fourier transform of equation $(2 \cdot 6.2 .1)$,

$\Rightarrow A(\vec{k})=-2 \pi J \vec{j} \cdot(i \vec{k},|\vec{k}|) e^{-|\vec{k}| z} \int_{D} d S \int_{0}^{h(\vec{r})} e^{|\vec{k}| z_{0}-i \vec{k} \cdot \vec{r}} d z$

(assuming constant magnetization) Integrating this equation with respect to $z$, the exponential term becomes

$$
\left(e^{|\vec{k}| h(\vec{r})}-1\right) /|\vec{k}|
$$

which can be expanded in a Taylor's series as

$$
(1 / .|\vec{k}|) \sum_{n=1}^{\infty} \frac{|\vec{k}|}{n !} h^{n} \cdot(\vec{r})
$$

substituting $(2.6 .2 .3)$ into $(2.6 .2 .2)$ and differentiating in the direction of the ambient field $(\vec{t})$ gives the expression for the anomalous field:

$$
H(\vec{k})=2 \pi J \vec{j} \cdot(i \vec{k},|\vec{k}|) \vec{t} \cdot(i \vec{k},|\vec{k}|) e^{-|\vec{k}| z_{0} \sum_{n=1}^{\infty} \frac{|\vec{k}|^{n-2}}{n !}\left[h^{n}(\vec{r})\right]}
$$


$-4$

Where $F[$ ] denotes Fourier transformation. For the case of constant magnetization the $z=0$ level can be chosen arbitrarily; Parker (1973) shows that for optimum convergence of the series in $(2.6 .2 .4)$, the $z=0$ level should -ibe placed at the average $h(\vec{r})$ value. Alteration of this "plane corresponds to the addition or subtraction of the effect of a constantly magnetised slab, which is zero at all 'points.

For the case of variable magnetization; the anomalous field becomes

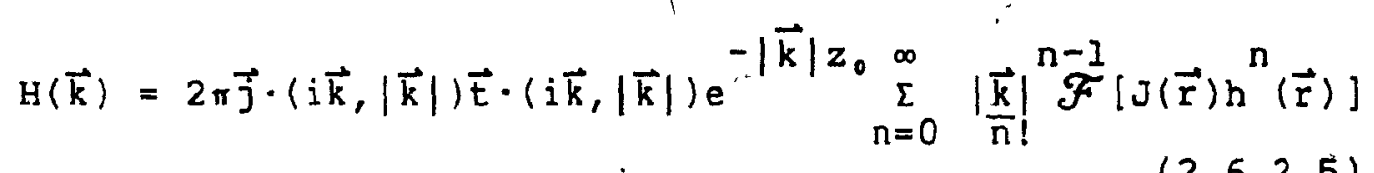

with the summation now beginning at zero. The extra term arises from considering the effect of a layer of unlimited depth extent: the limits of integration in (2.6.2.2) become $h(\vec{r})$ and $-\infty$. The resulting anomaly is monopolar and its average value is not zero, as it is for dipolar sources such as a constantly magnetised layer. The $n=0$ term in (2.6.2.5) represents the effect of a half-space bounded above by the plane $z=0$ and having a magnetization given by $J(\vec{r})$. The remaining terms then constitute a correction for topography of the layer's upper surface.

Equations $(2.6 .2 .4)$ and $(2.6 .2 .5)$ derive their efficiency by performing the integral in $(2.6 .2 .1)$ using Fourier transforms. Rapid algorithms are available to enable these 
expressions to be easily evaluated for map size data sets. Using Poisson's formula in the frequency domain, the gravity anomaly of a layer of constant or varying density can easily be derived. Similarly, the principle of superposition can be applied to find the effects of multi-layered models.

\subsubsection{The method}

In this section, a method for the inversion of magnetic data in terms of a single interface with constant magnetization is developed using the general theory summarised in section (2.5). Problem $T I$ is noñlinear due to the presence of varying depth to sources in the model, consequently the linearized form of the equations (2.3.4) is needed which requires an initial estimate of the topography $x^{0}$. This estimate can be interpreted as a priori information (Rodgers, 1976), so in the absence of such knowledge $x^{0}$ should be chosen in a way that it influences the form of subsequent iterates as little as possible. Such a choice is $x^{0}=0$. The a priori emphasis if any is on smooth'models, which is physically reasonable and important for the stability of the iterative process.

Linearising the forward problem with an initial estimate $h(\vec{r})=\overrightarrow{0}$ is simply done by taking the first term in the series for $H(\vec{k})$ (equation(2.6.2.4)) which gives

$$
H(\vec{k})=T(\vec{k}) \mathscr{F}[h(\vec{r})]
$$


where $T(\vec{k})$ is equal to the non-summed terms in $(2.6 .2 .4)$. The inverse transform of $(2.6 .3 .1)$ leads to

$$
B(\vec{r})=\mathscr{F}^{-1}[T(\vec{k})] \mathscr{F}[h(\vec{r})]
$$

which can be written in matrix form as

$$
y=E^{\star} T E x=A x
$$

$A$ is the Jacobian for the starting model $x^{\circ}$, and $T$ is a diagonal matrix with elements

$$
t_{i}=2 \pi J \vec{j} \cdot\left(i \vec{k}{ }_{i},\left|\vec{k}{ }_{i}\right|\right) \vec{t} \cdot\left(i \vec{k}_{i},\left|\vec{k}_{i}\right|\right) e^{-\left|\vec{k}_{i}\right| z_{0}}\left|\vec{k}_{i}\right|(2.6 .3 .4)
$$

which follows from (2.6.2.4). The Jacobian is circulant (e.g. Graybill, 1983, p.234) with elements $a_{i j}$ given by

$$
a_{i j}=\left.a^{\prime}(n+j-i)\right|_{\bmod n}
$$

where mod $n$ denotes modulo $n$ ( $n$ is the number of data) and $a^{\prime}$ is the Frechet derivative for the first parameter. A is therefore completely determined by its first row, that is, the Frechet derivatives of successive parameters are simply space shifted versions of each other. $E$ is the matcix formulation of Fourier transformation (e.g. Kanasewich, 1981, p.47); it is symmetric and unitary. From the properties of the Fourier transform, $E^{\star} E=I$ and $E^{-1}=E^{\star}$. The decomposition of $A$ in (2.6.3.3) follows directly from its 
circulant properties. Equation (2.6.3.3) is in the form of a nonlinear iterative inversion algorithm, that is, if the calculated responses of the model $x^{0}$ are $y^{0}$,

$$
y-y^{0}=A\left(x-x^{0}\right)
$$

When $x^{0}=0$, the calculated field is zero, and (2.6.3.5) reduces to $(2 \cdot 6 \cdot 3 \cdot 3)$. Solving for $x$ gives

$$
x=A^{-1} y=E^{*} T^{-1} E y
$$

if the appropriate inverses are assumed to exist. The iterative procedure of section (2.3) can then be implemented:

$$
x_{k+1}=x_{k}+A_{k}^{-1}\left(y_{o b s}-\hat{y}_{k}\right)
$$

with $\hat{y}$ being the calculated data from model $x_{k}$. From (2.6.3.1) this relation only holds at the first iteration using the decomposition in (2.6.3.3). The partial derivative matrix should be reevaluated at each $k$ using the current topography estimates $x_{k}$.

Equation (2.6.3.3) shows that the Jacobian $A$ has a simple decomposition which is independent of the topography. More importantly, its inverse is readily calculated by finding $\mathrm{T}^{-1}$, which being a diagonal matrix, only requires finding $1 / t_{i}$ where the $t_{i}$ are given in $(2.6 .3 .4)$. No matrix inversion is needed. This is not the case for (2.6.3.6). 
where the Jacobian is inverted at each iteration. For small maps of say $100 \times 100$ data, a matrix of dimensions. 10 000x10 000 would have to be inverted, a size that cannot be handled very easily. In contrast, the form of (2.6.3.3) allows large amounts of data to be processed as Fourier transformation is a simple and speedy operation. For this reason, the use of a constant $A$ is adopted for the method. The nonlinear algorithm used is then

$$
\begin{aligned}
x_{k+1} & =x_{k}+A^{-1}\left(y_{\text {obs }}-\hat{y}_{k}\right) \\
& =x_{k}+E^{\star-1} E\left(y_{\text {obs }}-\hat{y}_{k}\right)
\end{aligned}
$$

The initial iteration of $(2.6 .3 .7)$ gives the first linear approximate topography (FLAT) discussed in Chapter 1. The goodness of this approximation ensures that the parameter search is confined to a region of model space near the true solution.

In $(2.6 .3 .3)$ it is apparent that matrix $A$ has been factored into its singular value decomposition. $E$ and $E^{*}$ are therefore equivalent to eigenvector matrices corresponding to parameter and data space, respectively. The diagonal matrix $T$ contains the problem eigenvalues $\lambda_{i}$ (equation $(2.6 .3 .4))$. Each eigenvalue relates the spectral components. of $y$ to $x$ at wavenumber $\vec{k}$. This coincides exactly with Wiggins's (1976) interpretation of the decomposition of A: 
Considering (2.6.3.4), the magnetization and geomagnetic vector terms vary from 0 to 1 . Since all other terms are constant, the dominant factor controliing eigenvalue magnitude is the exponential term in $z_{0}$. As wavenumber increases, the eigenvalues diminish rapidly causing instability in the resulting inverse (see Figure 2.2). The parameter combinations that are poorly determined by the data are those corresponding to high wavenumber. The form of the eigenvalue spectrum exemplifies the well. known problem of short-wavelength instability in potential field

interpretation (Bullard and Cooper, 1948; Bott, 1973). In practice the observations provide no information regarding changes in topography over distances that are small with respect to the average depth to the interface.

The exponential term in the eigenvalues is the frequency domain form of the upward continuation operator for potential fields, (Dean, 1958). In order to mitigate the deleterious effects of small wavelength magnification, data is usuálly convolved with a low-pass filter before continuation. Viewing continuation as an inverse problem, the filtering is equivalent to using a damped approximate inverse. Since all procedures attempting to find source depth information implicitly involve downward continuation of the observed field, problem TI is ill-posed. As $z$ is the distance from the observation plane to the average layer topogaphy, the filtering required by $T I$ will be much more 


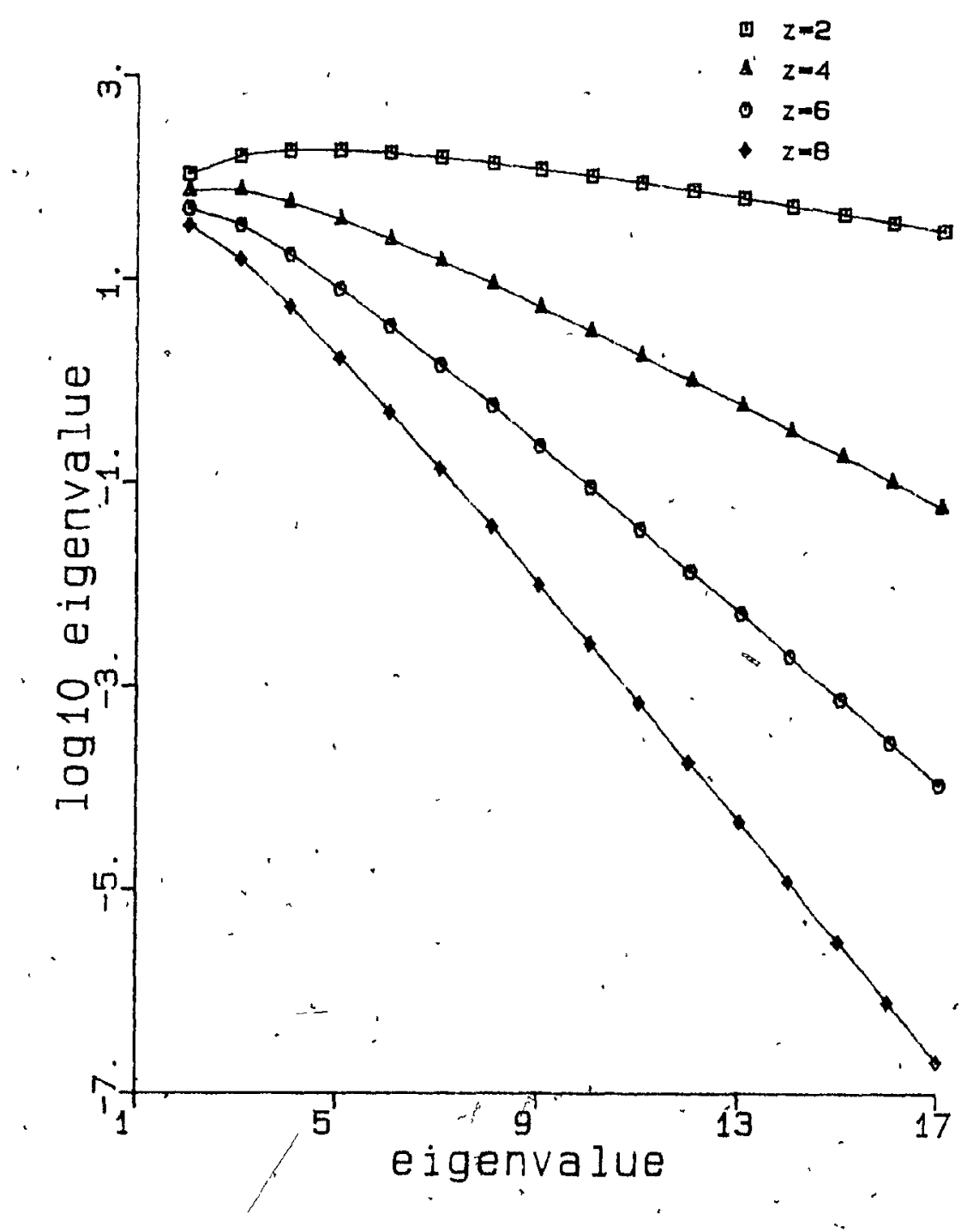

Figure 2.2 Eigenvalue spectra. for the one layer problem as a function of depth(z) for a layer with a magnetization of $1.2 \mathrm{~A} / \mathrm{m}$. 
severe than for continuation purposes.

The eigenvalue at wavenumber $\vec{k}=0$ is infinite, implying a long wavelength instability. This arises because the magnetic anomaly of an infinite horizontal slab is zero; thus causing the average topography value to "be indeterminate when the data is inverted. This is not a problem for sources with uniform magnetization, but if the magnetization distribution is sought in a layer of known topography, the zero eigenvalue leads to the existence of a non-constant magnetization function that can be added to a solution without affecting the observations (Parker and Huestis, 1974 ).

Problem TI is therefore faced with a zero eigenvalue at small wavenumbers and near-zero eigenvalues at large wavenumbers. In view of the remarks in section, (2.5.2) on the selection of the appropriate inverse, a combined generalized-Marquardt-Levenberǵ inverse is proposed as a remedy for the presence of zero and near-zero eigenvalues. The presence of the zero eigenvalue suggests a 'sharp cut-off' approach while the smooth (exponential) form of the remaining eigenvalues is best dealt with by damping. If $A^{+}$ is defined as the inverse employed, it has the form:

$$
A^{+}=E^{\star} \pi \mathrm{E}
$$

with, the elements of $\tilde{\lambda}$ being 


$$
\tilde{\lambda}_{1}=0, \lambda_{i}=\frac{\lambda_{i}}{\lambda_{i}^{2}+\theta} \quad i=2, n
$$

and $\lambda_{i}=t_{i}$ (equation $(2.6 .3 .4)$ ). Deleting the zero eigenvalue removes the average field value from the observations, hence no information is lost.

In section (2.5.2) it was shown how the use of a'damped approximate inverse or the removal of small eigenvalues caused a degradation in solution resolution but improved parameter reliability as expressed by the a posteriori parameter covariance matrix. The resolution and covariance matrix for the solution derived from application of the inverse of $(2.6 .3 .8)$ can be written explicitly by substituting $A^{+}$for $H$ in $(2.5 .2 .12)$ and $(2.5 .2 .13)$ giving

$$
\begin{aligned}
& R=A^{+} A=E^{\star} \frac{\Lambda}{\Lambda^{2}+\theta} \\
& C=A^{+} A^{+t}=\frac{E^{\star} \frac{\Lambda^{2}}{\left(\Lambda^{2}+\theta\right)^{2}}}{}
\end{aligned}
$$

Since $A$ and $A^{+}$were evaluated at a flat starting model, $R$. and $C$, are only valid for such a topography. They are completely determined by the eigenvalues (2.6.3.4) and are independent of the final solution from inversion. The rows of matrices $R$ and $C$ are identical except for a transiation in space. Consequently it is only necessary to compute the first row of $R$ or $C$ to completely specify the remainder of 
the matrix. Taking $R$, for example, the first row is found by taking the diagonal matrix $\Lambda^{2} /\left(\Lambda^{2}+\theta\right)$, putting this into column vector form and evaluating

$$
r_{1}^{t}=E^{*} \operatorname{col}\left(\Lambda^{2} /\left(\Lambda^{2}+\theta\right)\right)
$$

$r_{1}$ is the first row of $R$.

Figure 2.3a shows resolving kernels (rows of $R$ ), computed with the above method, as a function of layer depth (all other parameters constant) while Figure $2.3 \mathrm{~b}$ demonstrates the dependence of $R$ on layer magnetization. The influence of damping on resolution is illustrated in Figure 2.4.

As noted by IIkisik and Jones(1984), the use of a tapered cut-off on the eigenvalue spectrum divides the parameter combinations $\mathrm{V}^{t} \mathrm{x}$ into three groups:

(i) $\lambda^{2}>>\theta$ : parameters are important and well resolved

(ii) $\quad \lambda^{2}=\theta \quad$ : associated parameter combinations are marginally important

(iii) $\lambda^{2}<<\theta$ : parameters are poorly resolved

The covariance matrix (2.6.3.10) for case (iii) becomes

$$
C=\frac{V \Lambda^{2} V^{t}}{\theta^{2}}
$$

which provides variance estimates that are much' too small considering the poor resolution of the corresponding parameter combinations. The diagonal elements of $\mathrm{C}$ 
66

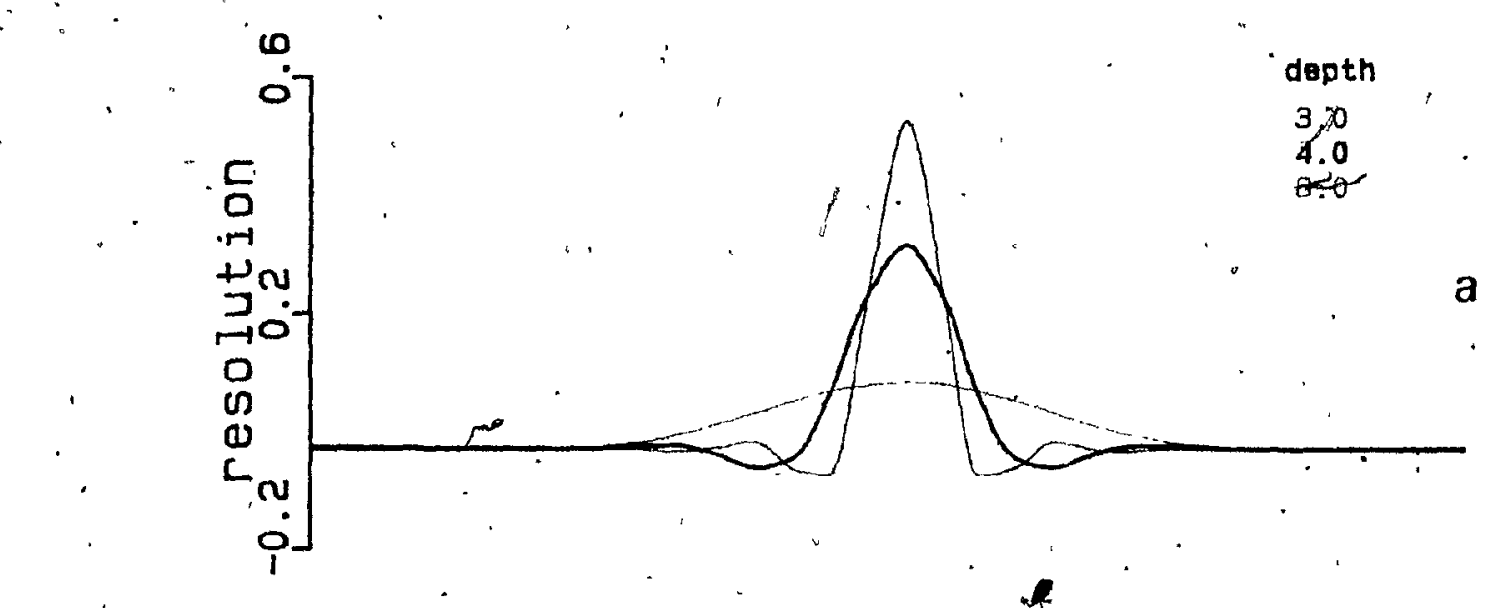

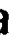

架

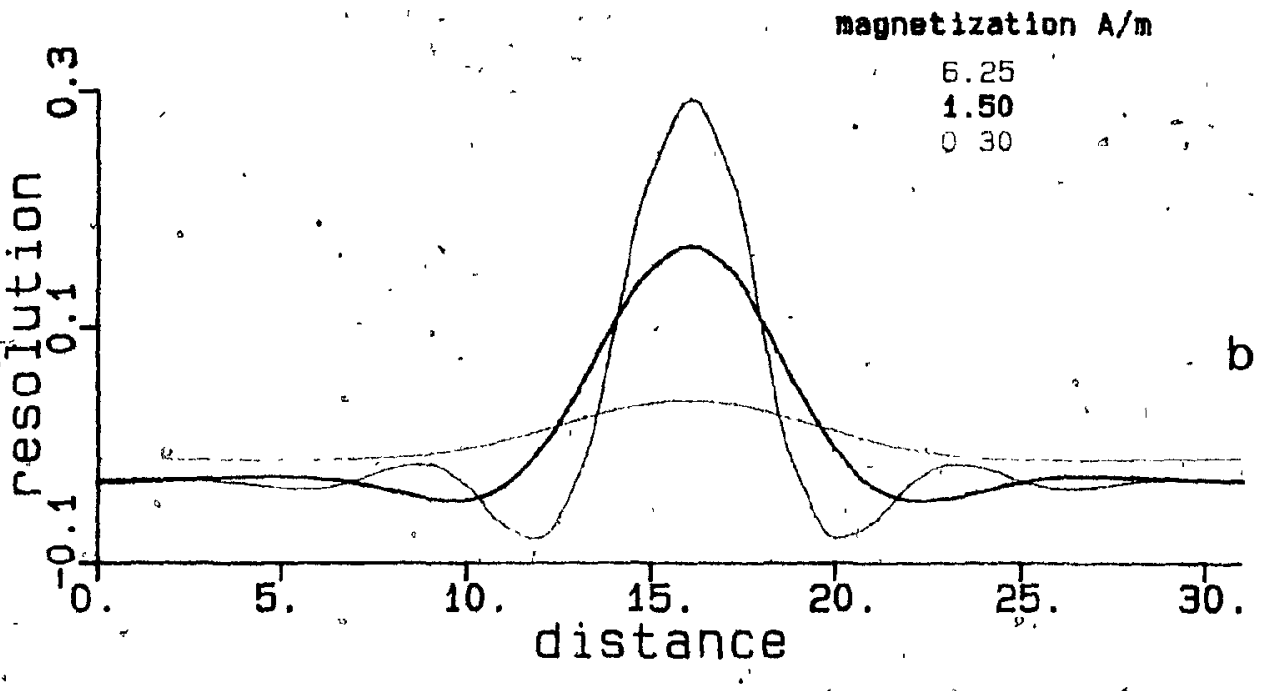

Figure 2.3 (a) Resolving kernels calculated from (2.6.3.9) for a layer with vertical magnetization of $1.2 \AA / m$ as a function of depth. (b) Resolving kernels calculated from (2.6.3.9) for a layer at depth 6 units as a function of magnetization. 
(1.

67 
(parameter variances) "should therefore be disregarded. Only variances, for case "(i) can be deemed as practical'measures of estimated parameter reliability.

So far only a two-dimensional (profile) case has been treated. For three-dimensional data in map form the (nxn). data matrix $Y$ can be written in the form

$$
Y=\dot{D} X^{0} D^{t}
$$

This is the " $3-D^{\circ}$ equivalent of the Iinearized forward. equation $(2.6 .3,3)$, with $X$ being the gridded topography values.。i is given by

$$
D=E^{\star} T^{1 / 2} E
$$

where $T$. is the eigenvalue matrix for the 2-D problem (equation $(2.6 .3 .4))$. Solving $(2.6 .3 .11)$ using least squares, the squared error $\|Y-E(X)\|^{\dot{2}}$ is to be minimized. This leads to the iterative algorithm

$$
x_{k+1}=x_{k}+D^{+}\left(Y-f\left(X_{k}\right)\right) D^{+t}
$$

with $D^{+}=E^{*} T^{-1 / 2} E$. .

The separable form in $(2.6 .3 .11)$ is only allowed when the Iinearized forward problem can be expressed in a linear shift-invariant convolutional form. When partial derivatives are taken at an acceptable solution this decomposition is 
lost and $x$ must be arranged into vector form (of length $n^{2}$ ) with a corresponding increase in size of the Jacobian to $n^{2} \times n^{2}$

If $x_{s}$ is the solution of a given two-dimensional-problem, then

$$
X_{S}=D^{+} Y D^{+t}
$$

and the resolution analysis of section (2.5) can proceed for such a case by substituting (2.6.3.11) into (2.6.3.12) giving

$$
\begin{aligned}
& x_{s}=D^{+} D x_{r} D^{t} D^{+t} \\
& \because=-R R_{r}^{t}
\end{aligned}
$$

with $x$ being the true solution and $R$, the resolution matrix. for the one-dimensional case. Instead of the resolving - kernels being vector quantities, they are now matrical. The resolving kernel for parameter $x_{i j}$ can be determined by forming the matrix $K$ given by

$$
k=r i^{t}
$$

where $r_{k}$ denotes the kth column of $R$. $k$ has a rank of one and a maximum eigenvalue of one for a perfectly resolved parameter. The separability of $K$ follows directly from that 
of the two-dimensional Jacobian.

\subsubsection{Iterative procedure}

In section $(2.3)^{n}$ it was pointed out that with any iterative algorithm there is the question of whether the acceptable solution reached corresponds to a global or local minimum of the objective function. No theory is yet available to answer this, however confidence can be instilled by reaching the same solution when the procedure is initialised with distinctly different models.

Whether or not conyergence is obtained can be investigated by considering the change in RMS error $\left(c^{2}\right)$ from one iteration to the next. "The RMS error at iteration $\vec{k}$ is given by

$$
\varepsilon_{k}^{2}=|| y_{\text {obs }}-\hat{y}_{k}||
$$

From $(2.6 .2 .4)$ the calculated response $\hat{y}$ can be written

$$
\hat{y}_{k}=x_{k}-\frac{\mathcal{O}\left(x_{k}^{2}\right)}{k}
$$

where $\mathcal{O}\left(x^{2}\right)$ are the remaining terms in the series (equation $(2.6 .2 .4))$. The general algorithm is

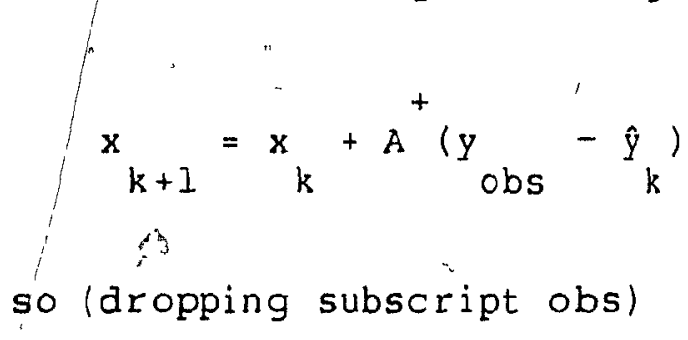




$$
\begin{aligned}
& z_{k+1}^{2}=|| y-A x_{k+1}-O\left(x_{k+1}^{2}\right)|| \\
& =|| y-A\left(x+A+\left(y-A x-O\left(x^{2}\right)\right)-O\left(x_{k}^{2}\right)|1|\right.
\end{aligned}
$$

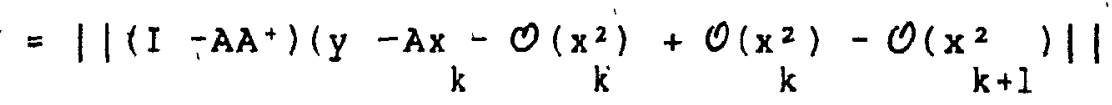

$$
\begin{aligned}
& \leq|| I-A A^{+}||\left(\varepsilon_{k}^{2}\right)+|| \mathcal{O}\left(x_{\hat{k}}^{2}\right)-\underset{k+1}{-O}\left(x^{2}\right)|| \\
& c_{k+1}^{2} \leq \varepsilon_{k}^{2}+|| \underset{k}{O}\left(x^{2}\right)-\mathcal{O}\left(x_{k+1}^{2}\right)||
\end{aligned}
$$

The last step follows from the SVD of the matrix $\left(I-\mathrm{AA}^{+}\right)$,

$$
\begin{aligned}
I-A A^{+} & =I-U \wedge V^{t} V \pi U^{t}{ }^{\prime} \\
& =U U^{t}-U \wedge \pi U^{t} \\
\text { so } & =U(I-\wedge \pi) U^{t} \\
& =U-A A+|| l|| I-\wedge \pi||
\end{aligned}
$$

knowing that $\|U\|=\| U^{t}||=1$. Now, using the spectral radius $\rho()$,

$$
\begin{aligned}
\| I-\Lambda \pi|| & \leq p(I-\wedge \pi) \\
& =\max _{i}\left(1-\lambda^{2} /\left(\lambda^{2}+\theta\right)\right. \\
& =\min _{i} \lambda^{2 /\left(\lambda^{2}+\theta\right)} \\
&
\end{aligned}
$$

So

$$
|| I-A A+|| \leq 1
$$

Equation $(2.6 .4 .2)$ shows a reduction in the objective function is achieved as long as the nonlinear terms are 
small. From $(2.6 .4 .4)$, as $\theta \rightarrow 0,-\rho(I-\wedge \pi)-1$, as $\theta$ increases, $\rho(I-\Lambda \dot{\lambda})-0$ and

$$
c_{k+1}^{2} \leq||\left|\mathcal{O}\left(x_{k}^{2}\right)-\mathcal{O}\left(x_{k+1}^{2}\right)\right| \mid
$$

This is the case of steepest descent for which convergence is guaranteed.

Defining a final solution or an end to iterating, is achieved by specifying one or more termination criteria. In the proposed method, the iterative process is stopped when one of the following criteria is satisfied

$$
\begin{aligned}
\left\|x_{k}-x_{k-1}\right\| & \leq 6 \\
c^{2} & >0.9 c_{k-1}^{2} \\
k & \leq c^{2} \\
k & \text { est } \\
c^{2} & >\varepsilon_{k-1}^{2}
\end{aligned}
$$

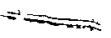

The, Eirst two measures indicate the iterative search is close to a solution while (2.6.4.8) shows the algorithm is diverging due to either a starting, model far from the solution or poorly chosen auxiliary parameters such as the damping factor, $\theta$. Condition $(2.6 .4 .7)$ ensures the algorithm is stopped when the calculated "data fit the observations to 
within their estimated error ( $c^{2}$ est).

\subsubsection{Residuals calculation}

The forwara problem of calculating the field produced by a model is solved by $(2.6 .2 .4)$. Even though this method is fast, a further increase in computational speed can be gained by calculating the latest field $\mathrm{H}_{k+1}$ as the sum of the previously determined, $H_{k}$ and a perturbation $\Delta H_{k}$ resulting from the most recent, parameter correction $\Delta x_{k}$. This is in contrast to the standard procedure of calculating $H_{k+1}$ directly from $x_{k+1}$. From $(2.6: 2.4)$ the field from model $x_{k}$ is

$$
H_{k}^{\circ}=T(\vec{k}) \sum_{n=1}^{\infty}\left|\frac{\vec{k}}{n}\right| \mathcal{F}\left[x_{k}^{n}\right]
$$

where $T(\vec{k})$ are the non-summed terms in $(2.6 .2 .4)$. If $x_{k+1}=x_{k}+\Delta x_{k}$, the series term, in $(2,6.5 .1)$ becomes

$$
|\vec{k}| \mathscr{F}[x+\Delta x]+\left.\frac{\mid \vec{k}}{2}\right|^{2} \mathscr{F}\left[(x+\Delta x)^{2}\right]+\ldots(2.6 .5 .2)
$$

Subscript $k$ has been dropped for convenience. Neglecting terms of $\mathcal{O}\left(\Delta \mathrm{x}^{2}\right)$ in $(2.6 .5 .2)$ gives

$$
|\vec{k}| \mathscr{F}[\mathrm{x}]+\left|\frac{\vec{k}}{2}\right|^{2} \mathscr{F}\left[\mathrm{x}^{2}\right] \ldots+|\overrightarrow{\mathrm{k}}| \mathcal{F}[\Delta \mathrm{x}]+\left|\frac{\vec{k}}{2}\right|^{2} \mathscr{F}[2 \mathrm{x} \Delta \mathrm{x}] \ldots
$$

So the correction due to the parameter change $\Delta x_{k}$ becomes 
(collecting terms in $\Delta x_{k}$ ),

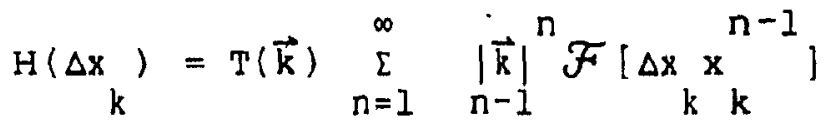

From the convergence properties of this type of series (Parker, 1973), the number of terms required in (2.6.5.3) is smaller than that of $(2 \cdot 6 \cdot 5.1)$.

2.6.6 Partial derivative calculation

The partial derivative matrix $A$ used in the method is only valid around the model $h(\vec{r}) \equiv \overrightarrow{0}$. Solution appraisal is approachable only when the partial derivatives are computed at the acceptable solution $\left(h^{\prime}(\vec{r})\right)$. Once this has been found using the algórithm (2.6.3.7), a new $A$ can be found as follows. The Fourier transform of the anomaly due to a layer $h(\vec{r})$ is

$$
H(\vec{k})_{h=h(\vec{r})}=C e^{-|\vec{k}| z_{0}} \int_{D} d S e^{-i \vec{k} \cdot \vec{r}}\left(e^{|\vec{k}| H(\vec{r})}-I\right)
$$

where

$$
C=2 \pi J \vec{j} \cdot\langle i \vec{k},|\vec{k}|) \vec{t} \cdot(i \vec{k},|\vec{k}|\rangle /|\vec{k}|^{2}
$$

Expanding the exponential term in $(2.6 .6 .1)$ around the acceptable solution $h^{\prime}(\vec{r})$

$$
\begin{aligned}
e^{|\vec{k}| h(\vec{r})} & =e^{|\vec{k}|\left(h^{\prime}(\vec{r})+\Delta h(\vec{r}) \mid\right.} \\
& =e^{|\vec{k}| h^{\prime}(\vec{r})}\left(1+|\vec{k}| \Delta h(\vec{r})+\left|\frac{\vec{k}}{2}\right| \Delta h^{2}(\vec{r})+\ldots\right)
\end{aligned}
$$


Neglecting terms of order $(\Delta h(\vec{r}))$ and above, (2.6.6.I) becomes

$$
\begin{aligned}
H(\vec{k}){ }_{h=h(\vec{r})} & =c e^{-|\vec{k}| z_{0}} \cdot \int_{D} \text { as } e^{-i \vec{k} \cdot \vec{r}}\left[e^{|\vec{k}| h^{\prime}(\vec{r})}(1+|\vec{k}| \Delta h(\vec{r}))-1\right] \\
& =H(\vec{k}) \\
& =h_{h=h^{\prime}(\vec{r})} \\
& +C e^{-|\vec{k}| z_{0}}|\vec{k}| \int_{D} d S e^{-i \vec{k} \cdot \vec{r}} e^{|\vec{k}| h^{\prime}(\vec{r})}|\vec{k}| \Delta h(\vec{r})
\end{aligned}
$$

The second term in (2.6.6.2) then represents the change in the calculated field due to the perturbation $\Delta h(\vec{r})$. Expanding the exponential term in $h^{\prime}(\vec{r})$ in $(2: 6.6 .2)$ in a Taylor's series and using the convolution theorem, the calculated field change can be expressed as

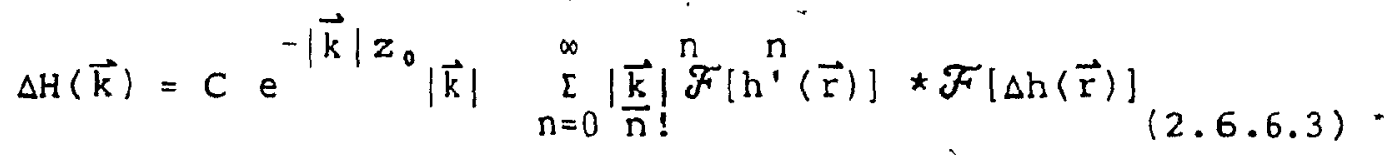

where * denotes convolution. Writing $(2.6 .6 .3)$ in matrix form

$$
\Delta H=W K V E \Delta
$$

$E$ is the Fourier transform matrix and

$$
\begin{aligned}
& W=\operatorname{diag}\left(c e^{-|\vec{k}| z_{0}}\right) \\
& K=\operatorname{diag}(|\vec{k}|) \\
& V=I+K Q_{1}+K^{2} Q_{2}+K^{3} Q_{3}+\ldots .
\end{aligned}
$$


where $\mathrm{Q}_{\mathrm{n}}$ has, the form:

$$
Q_{n}=\left[\begin{array}{cccccc}
h_{0}^{n} & h_{1}^{n} & h_{2}^{n} & \cdot & \cdot & h_{N}^{n} \\
h_{N}^{n} & h_{0}^{n} & h_{1}^{n} & \cdot & \ddots & h_{N-1}^{n} \\
h_{N-1}^{n} & \cdot & \cdot & \cdot & \cdot & \cdot \\
0 & \vdots & \cdot & \cdot & \cdot & \cdot \\
h_{1}^{n} & h_{2}^{n} & h_{3}^{n} & \cdot & \cdot & \cdot h_{0}^{n}
\end{array}\right]
$$

and $N$ is the number of data (and parameters) and $h \stackrel{n}{j}$ represents the Fourier transform of the topography $h^{n}(\vec{r})$ at wavenumber $j$. The convergence of the series for $v$ follow.s from the equivalence (apart from a factor of $|\vec{k}|$ and $a$ translation in the wavenumber domain) between the columns of $Q_{n}$ and the series in equation (2.6.2.4). Inverse transforming $(2.6 .6 .3)$,

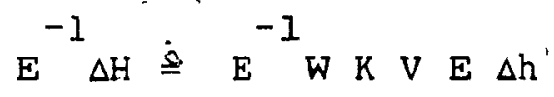

The matrix $\mathrm{E}^{-7}$ WKVE then represents the partial derivative matrix with elements

$$
a_{i j}=\left.\frac{\partial b(r)_{i}}{\partial h(r)_{j}}\right|_{h=h^{\prime}(\vec{r})}
$$

As noted earlier, this matrix must be inverted leither explicitly or by solving a system of simultaneous equations) for the process of solution appraisal to proceed. If certain features in the estimated solution need to be assessed as 
regards, their resolvability by the data, it is not necessary to construct resolving kernels at everý point in the data set, just those of interest. Due to the size of the Jacobian, the former approach would be formidable anyway, unless the data is divided into smaller units and treated separately.

Equation $(2.6 .6 .4)$ can therefore be used for quick evaluation of partial derivatives for localized areas whose size will be governed by the features of interest and computational considerations.

\subsection{Multilayer inversion}

\subsubsection{Introduction}

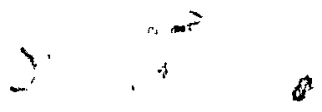

The most common application of the interpretation of data in terms of a single interface arises when a magnetic crystalline basement is overlain by non-magnetic sedimentary rocks. Such an analysis provides important geological and geotectonic information concerning the structural imprinting of basement features, on the sedimentary cover. However, problems arise when the effects of such features are obscured'by the presence of intrusives or volcanic flows within the sedimentary sequence. The resulting interpretive problem becomes more underdetermined and some, kind of separation of the two effects is needed for the modelling problem to become tractable. Unfortunately, in view of the 
spectral overlap of sources occuring at different depths (e.g. Bhattacharyya, 1966), a perfect separation is not possible. This condition has led to the subjective approach of simply dividing the observed field into low and $h$ igh frequency parts and interpreting each one exclusively (Lehmann, 1970; Sprenke and kanasewich, 1982). A more. accura method has been advocated by spector and Parker (1977) who separate the two effects based on power spectral analysis of the observations.

The above methods treat the fields, arising from deep and shailow sources separately once the division is made: A more objective approach is to treat the observed field as a single entity during the whole interpretive process, especially' since the problem is nonlinear. This can be achieved by extending the theory of section (2.6) to the multilayered model case using the philosophy of Lanczos (1963). The multilayered topography inversion problem can thus be examined in the light of its singular value decomposition, for which it is shown an explicit factorization exists.

\subsubsection{Multilayer singular value decomposition}

For simplicity's sake only two layers are modelled initially; the results are then generalized to the n-layered problem. From (2.6.3.3), the'linearized forward problem for two layers can be expressed as 


$$
y^{\prime}=E^{\star} F E E^{*}+E^{\star} G E g
$$

$E$ and $E^{\star}$ are the usual Fourier transform matrices, , $f$ and $g$ * are the topographies of the two layers with their corresponding eigenvalue matrices being $F$ and $G$, respectively. $F$ and $G$ have the form of $(2.6 .3 .4)$ with the appropriate parameters substituted for each layer. Equation. (2.7.2.1) becomes, in partitioned form

$$
\begin{aligned}
y & =A x \\
& =\left[\begin{array}{lllllll}
E^{\star} & F & E & E^{\star} & G & E
\end{array}\right]\left[\begin{array}{l}
f \\
g
\end{array}\right]
\end{aligned}
$$

If $y, f$ and $g$ are $n$-vector's, matrix $A$ is of dimensions $n \times 2 n$ and (2.7.2.2) represent an underdetermined problem. Forming the coupled eigensystem (Lanczos, 1963, Ch. 3)

$$
S w=\lambda w
$$

where'S is Hermitian and

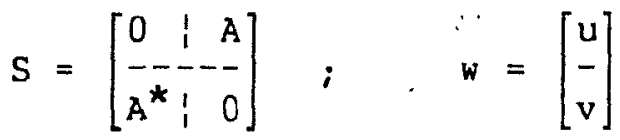

The $2 n$-vector $w$ is partitioned into two sets of eigenvectors. , coupled as follows:

$$
\begin{aligned}
A^{\star} A \mathrm{~V} & =\lambda^{2} \mathrm{v} \\
\text { A }^{*} \mathrm{u} & =\lambda^{2} \mathrm{u}
\end{aligned}
$$


Substituting the partitioned form for A gives

and

$$
{ }^{\star} A=\left[\begin{array}{c:c}
E^{*}{ }^{2} & E^{*} F G E \\
\hdashline E F G E & E G^{2}
\end{array}\right]
$$

$$
A A^{*}=E^{*}\left(F^{2}+G^{2}\right) E \quad \because
$$

$(2,7,2,4)$

From (2.7.2.3), using the complete set of eigenvectors, $U$,

?

$$
\begin{aligned}
A A^{*} & =U \Lambda^{2} U^{*} \\
& =E^{*}\left(F^{2}+G^{2}\right) E
\end{aligned}
$$

following (2.7.2.4). Equating terms above, it follows that

$$
U=E^{*}, U^{*}=E, \Lambda^{2}=\left(F^{2}+G^{2}\right)
$$

Similarly, from $(2,7.2 .3)$ and $(2.7 .2 .5)$

$$
\begin{aligned}
A^{*} A & =V \wedge^{2} V^{\star} \\
& =V\left(F^{2}+G^{2}\right) V^{*}
\end{aligned}
$$

Equating (2.7.2.6) with the partitioned form of $A^{*} A$, gives

$$
V=\left[\begin{array}{cc}
{ }^{*} & F /\left(F^{2}+G^{2}\right)^{1 / 2} \\
\hdashline{ }^{*}-\left(E^{2}+G^{2}\right. & \\
E^{1 / 2} & G /(F+G)^{2}
\end{array}\right]
$$

The eigenvectors of $\mathrm{V}$ are orthonormal but do not form a complete set. The singular value decomposition for $A$ in the 
two layer case is then

$$
\begin{aligned}
A & =U \wedge v^{t} \\
& =[E]\left[\left(E^{2}+G^{2}\right)^{1 / 2}\right]\left[F /\left(F^{2}+G^{2}\right)^{1 / 2} E\left[G /\left(F_{c}+G^{2}\right)^{1 / 2} E\right]^{t}\right. \\
& =\left[E^{*} F\left[E^{*} E^{*}\right]\right.
\end{aligned}
$$

The natural or Lanczos inverse of $A$ is.

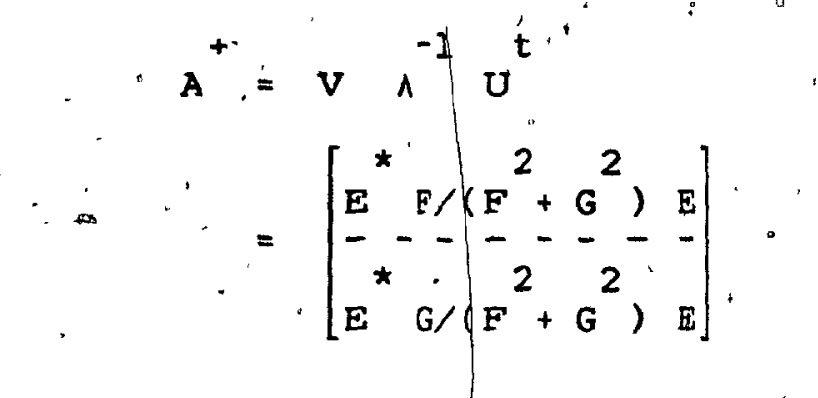

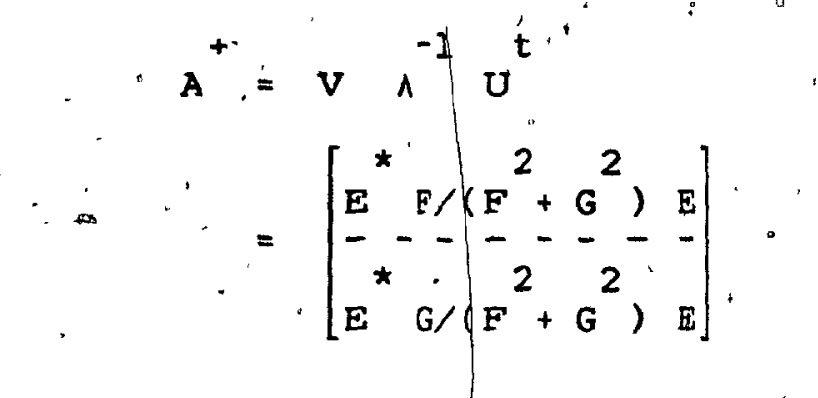

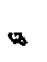

Expression $(2.7 .2,8)$ for the inverse of A can also be' derived u'sing cline's (1964) formula for the generalized or pseudo-inverse of an arbitrary partitioned matrix. Cline . shows that if $A$ is partitioned as

$$
A=\left[\begin{array}{lll}
X & 1 & Y
\end{array}\right]
$$

its generalized inverse, $A^{+}$, is given by

where

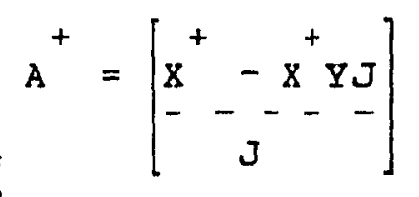

$$
\begin{aligned}
& J=C^{+}+\left(I-C^{+} C\right) K Y^{t} X^{+t} X^{+}\left(I-Y C^{+}\right) \\
& C=\left(I-X X^{+}\right) Y
\end{aligned}
$$

and 


$$
\left.K=\left(I+\left[X^{+} Y\left(I-C^{+} C\right)\right]^{t}\left[X^{+} Y\left(I-C^{+} C^{+}\right)\right]\right)^{--}\right]
$$

Substituting the decomposition for $A$ into $(2,7.2 .9)$ gives the result in $(2.7 .2 .8)$. Additionally, $(2.7 .2 .8) \mathrm{can}$ be derived by forming the least squares inverse for the underdetermined case, $A^{t}\left(A A^{t}\right)^{-1}$, using the known form of $A$ and $A^{t}$. A straightforward extension to the $n$-layered case gives the inverse

$$
{ }^{+}=\left[\begin{array}{cccc}
- & - & - & - \\
- & - & - & - \\
* & & \\
E & -I_{i} & E \\
\hdashline n & 2 \\
L & L \\
i=1 & i \\
-- & - & - \\
- & - & - & -
\end{array}\right]
$$

where $L$ is the eigenvalue matrix for the ith layer.

The above- decomposition (equation $(2.7 .2 .7\rangle)$ has the expected properties of an underdetermined problem. If the rank of $A$ is $n$, $U$ space is complete but $V$ space is deficient (section $(2.5 .2)$ ). Parameter combinations that have no effect on the observations ( $V_{0}$ space) can be determined by simply taking linear combinations of the columns of the $\operatorname{matrix}\left(I-V V^{t}\right)$.

The measures used in solution appraisal can be written explicitly in terms of the eigenvalue matrices, F and G:

$$
S=A^{+}=U^{t^{+}}=I \cdot \quad \text { (Information density) }
$$




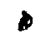

$$
\begin{aligned}
& R=A^{+} A=V V^{t} * I \quad \text { (Resolution) }
\end{aligned}
$$

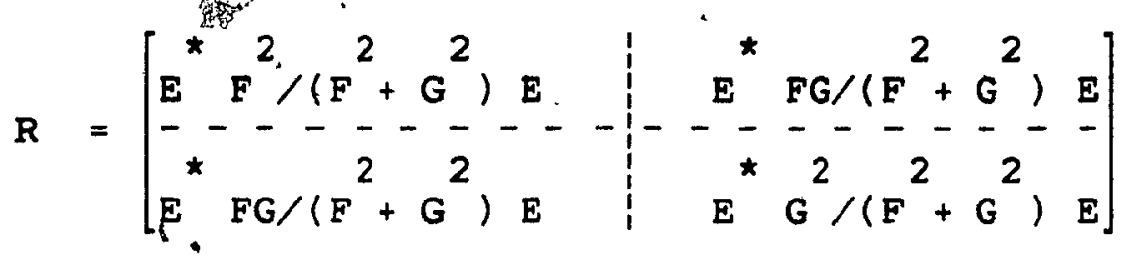

$$
\begin{aligned}
& C=A^{+} A^{+t}=V \wedge^{-2} V^{t} \quad \text { (Covariance) }
\end{aligned}
$$

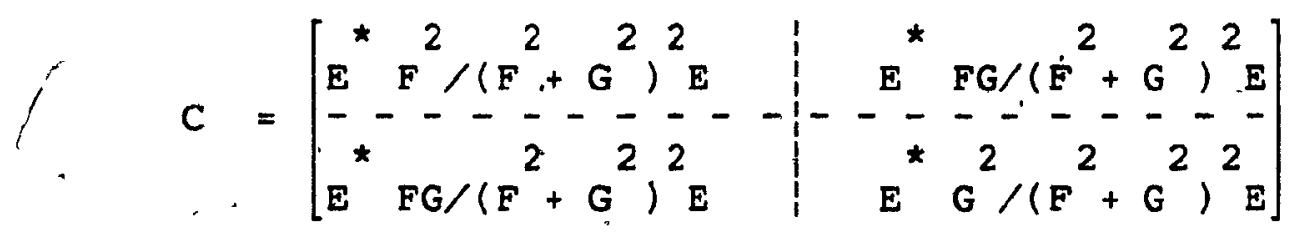

The form of the resolution matrix $R$ shows that for perfect resolution of parameters, $F$ and $G$ must be uncorrelated with no overlap in the frequency domain. Since this is not theoretically possible due to the existence of. potential fields at all frequencies irrespective of source depth, degraded resolution must always be expected. Figure 2.5 exemplifies the poor control on parameter resolution for the lower layer when layer separation is increased. Figure 2.6 shows that as the layers get closer, resolution improves but the upper layer resolution is degraded by sidelobes. The resolving kernels are independent of the absolute layer depths but depend on layer separation. Similarly, only the ratio of layer magnetizations is relevant to resolution.

It should be noted that in Figures 2.5 and 2.6 , all kernels were calculated assuming no damping, all éigenvalues 

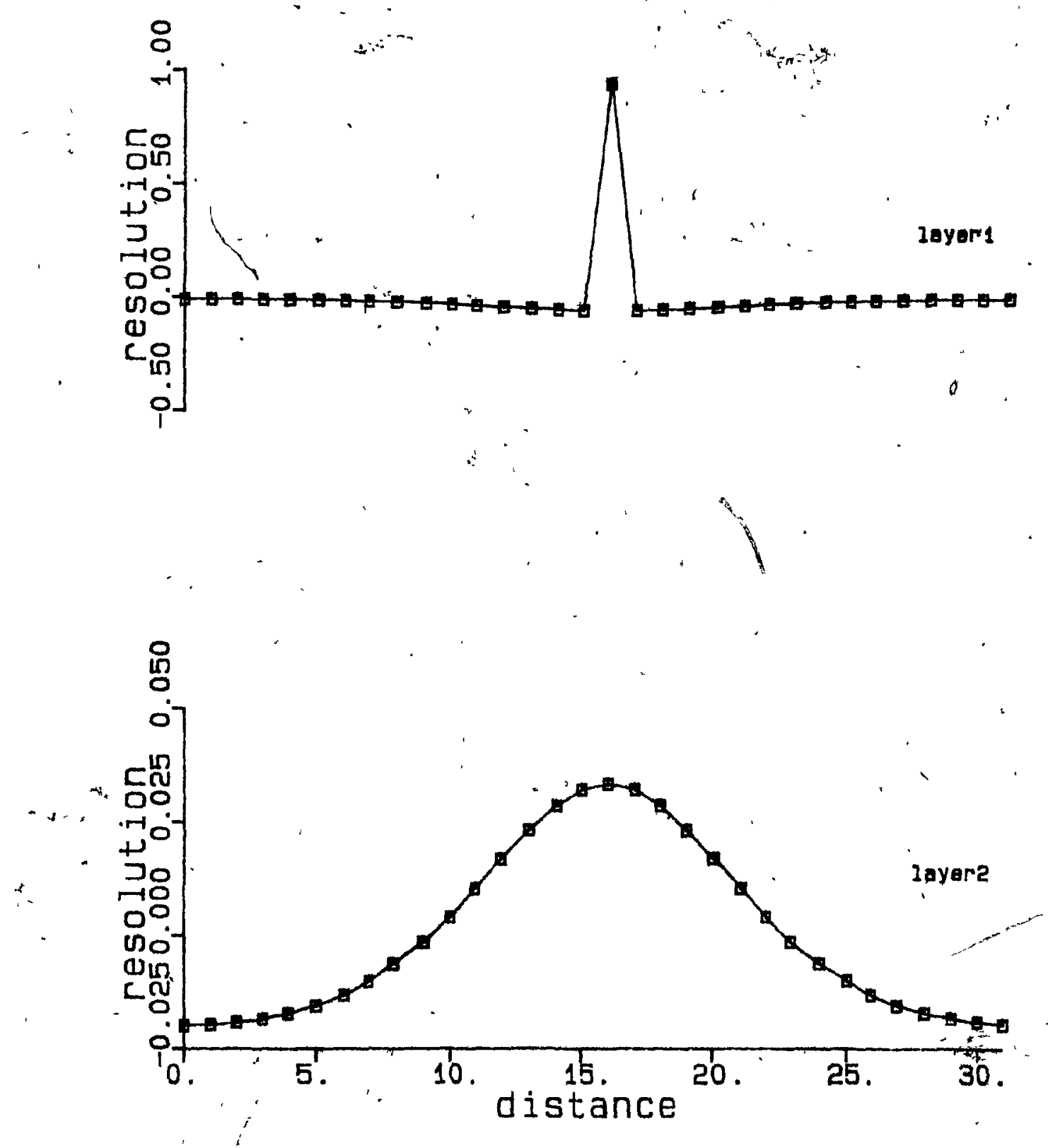

Figure 2.5 Resolving kernels. for the two layer problem. Layer depths and magnetizations are 1.5, 6.0 arbitrary units and $0.6,1.2 \mathrm{~A} / \mathrm{m}$ respectively. 

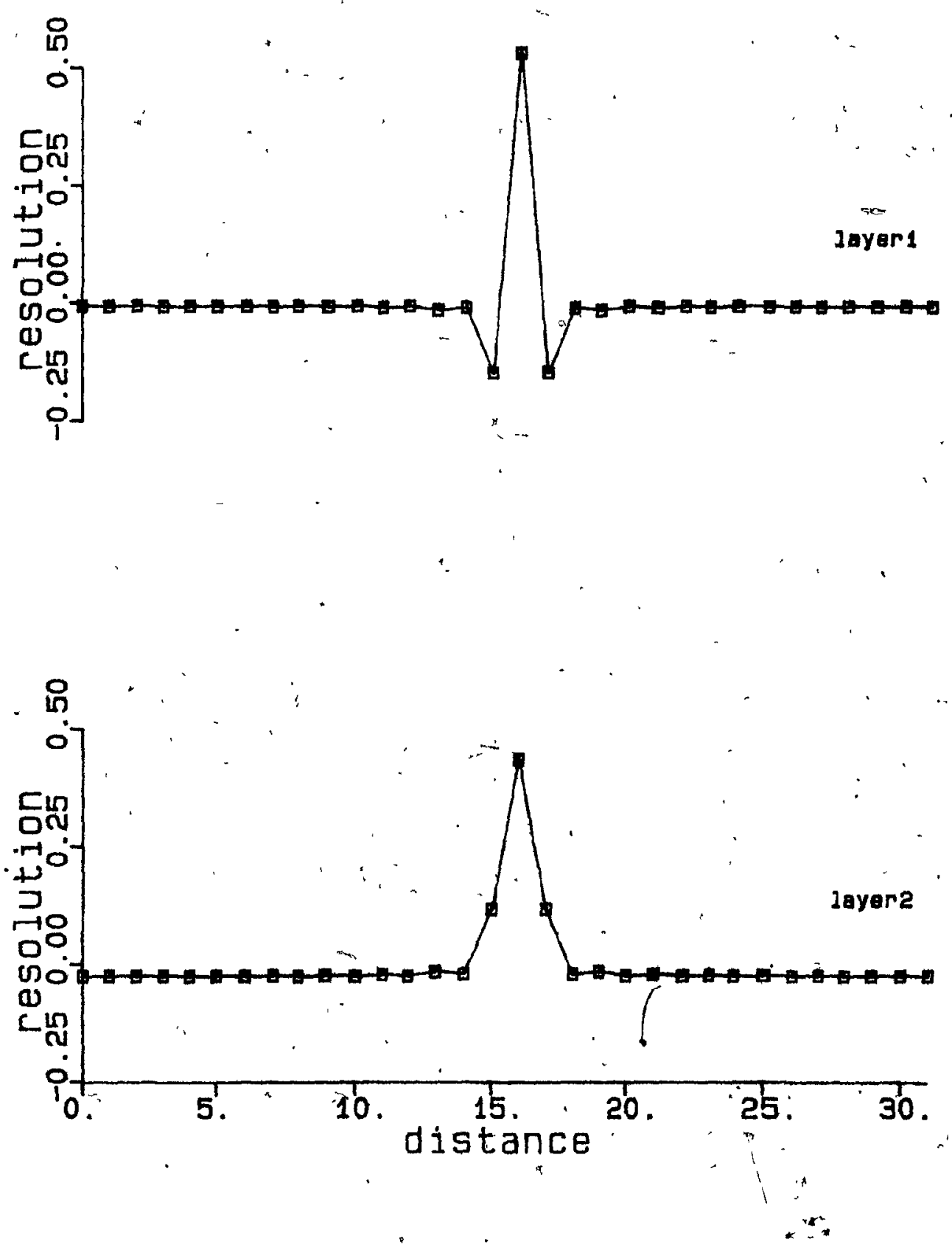

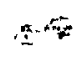

Figure 2.6 Resolving kernels for the two layer problem. Layer depths and magnetizations are $3.0,3.5$ arbitrary units and $0.6,1.2 \mathrm{~A} / \mathrm{m}$ respectively. 
except the zero one have been retained in the solution giving the bèst resolution possible.

The effects of damping on parameter resolution and covariance follows by analogy to the one-layer case (section 2.6.3). The two layer inverse with damping becomes

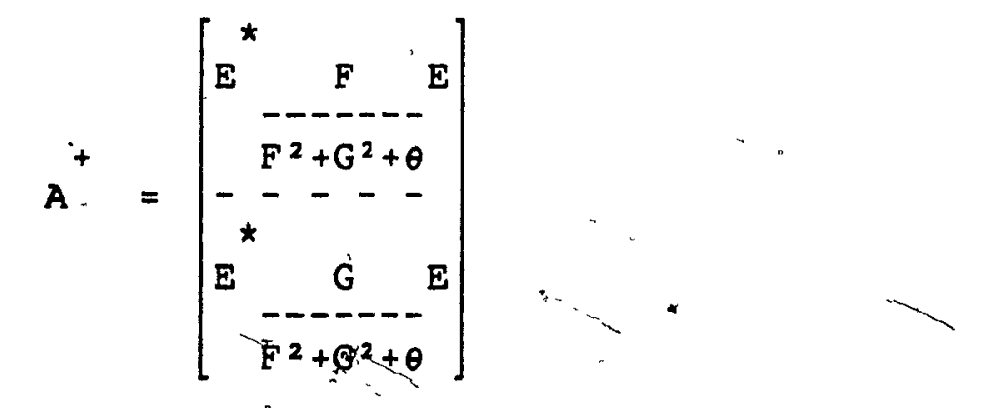

where $\theta$ is the damping factof. The corresponding expressions for $R$ and $C$ foliow by substituting the decompostion for $A^{*}$ into $R=A+A$ and $C=A+A+t$.

it is illuminating to investigate the nature of the óbserved field separation imparted by the decomposition (2.7.2.7). Applying the 2-layer inverse (equation (2.7.2.8)) to the field b gives, for the first approximate topography.

$$
\begin{aligned}
& {\left[\begin{array}{l}
f^{\prime} \\
g^{\prime}
\end{array}\right]=A^{+} b}
\end{aligned}
$$

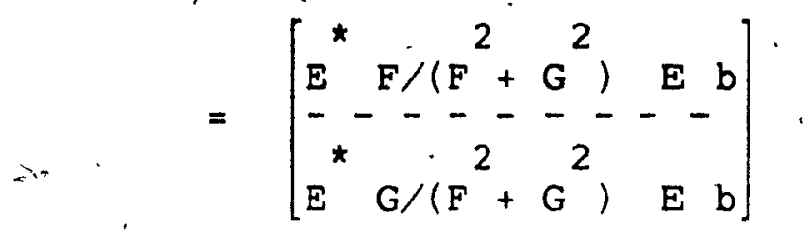

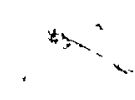

Considering just the top layer, the expression for $f$ ', the topography, is equivalent to 


$$
E^{\prime}=E^{*} F^{-1} E\left[E^{*} F^{2} /\left(F^{2}+G^{2}\right) E b\right]-
$$

The non-bracketed tẹm. ' $f_{i s}$ simply the one-layer inverse (equation $(2 \cdot 6.3 .7)$ ), while the term in brackets can be regarded as transformed or filtered datia:

$$
b^{\prime}=E^{*} E^{2} /\left(F^{2}+G^{2}\right) E b=W b
$$

The form of $W$ is the familiar one of a wiener filter, where $F^{2}$ is interpreted as the signal energy spectrumband $\mathrm{G}^{2}$ is the noise energy spectrum. Squaring the eigenvalue matrices $F$ and $G$ gives the energy spectrum of a random distribution of dipoles located at a constant depth. The two-layer solution in $(2.7 .2 .11)$ is then equivalent to wiener filtering the data (or residuals in subsequent iterations. and obtaining the one-layer solution from the filtered field. It should be noted that the filter $W$ can be derived from Backus-Gilbert theory through the minimisation of the quadratic form (2.5.1.7) using the Dirichlet criterion for the simple case of data corrupted by coloured noise. From this viewpoint, the wiener filter is just one member of a class of noise reduction filters corresponding to different values of the-trade-off parameter $r$. The usual form above arises when $r$ is chosen as $45^{\circ}$, that is, equal weighting to. resolution and noise surpression. 


\subsubsection{Reparameterization and problem eigenvalues}

Matrix V, the parameter eigenvector matrix, acts on the real parameters to produce a ranking of the parameter combinations whose influence on the data (and the solution) is measured by the associated eigenvalue magnitudes. By studying the general form of this operator, the importance of these parameter components can be evaluated as a function of layer properties. For the following discussion, the geomagnetic and magnetization vectors are assumed to be the same for both layers. The eigenvalues in $F$ and $G$ are then completely determined by specifying the average depth of the layer and its magnetization contrast. Figure 2.7 shows the effects of reparameterization in the frequency domain as a function of the distance between layers. The form of $V$ is unaltered by the absolute depths of the layers, it depends only on their separation. Figure 2.7 shows that reparameterization for the top layer (layer i) is essentially a high-pass filtering operation while for layer 2 , only the low frequencies are passed. The severity of the filtering for each layer is increased as the layer separation increases. Figure 2.7 , as expected, shows that an increase in the lower layer's magnetization causes more weight to be assigned to that layer in the observed field separation, and the low-pass filtering effect is diminished. Thus layer separation and the ratio of upper and lower layer magnetization are the dominant factors controlling the 

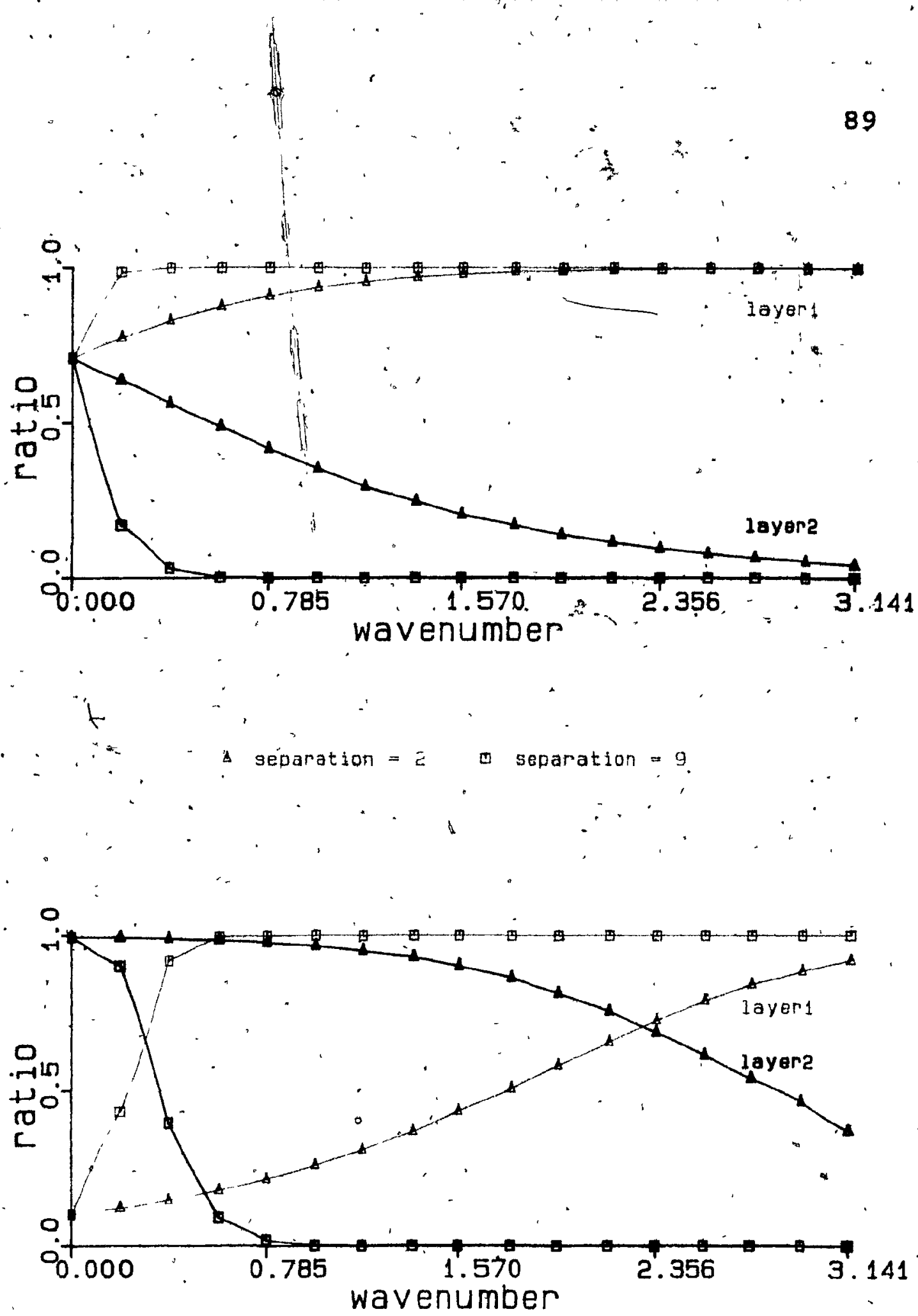

Figure. 2.7 Reparameterization in the wavenumber domain for the two layer problem. The ratios $F / V\left(F^{2}+G^{2}\right)$, and $G / V /\left(F^{2}+G^{2}\right)$ (equation (2.7.2.7)) are plott"ed for the upper and lower layers respectively. In (a) the layer magnetizations are equal. In (b) the lower layer magnetization is 10 times that of the upper layer. In both (a) and (b) the dependence of the reparameterization on the distance between layers (separation) is illustrated. 
wavelength separation between the two layers.

The gross effects of the reparameterization are intuitively reasonable considering the relationship between frequency content and source depth (Bhattacharyya, 1966).. What is not expected, is that the exclusion of high frequencies from the lower layer's reparameterization ensures its stable inversion at the expense of the top layer's stability. This is in contrast to one-layer inversion, where layers at greater depths are accompanied by increased instability as shown by an increase in the number of near-zero eigenvalues (Figure 2.2).

The behaviour of the problem eigenvalues (equation $(2.7 .2 .7))$ or the n-layered model is similar to the single interface problem with small eigenvalues occuning at large wavenumbers. Figure 2.8 shows their behaviour for the two-layer problem. The general form of the spectra are determined by the top layer depth and magnetization. Keeping the top depth constant and deepening the bottom layer results in only a small decrease at low wavenumbers. Tables 2.1 and 2.2 give a quantitative assessment of the conditioning of matrix $A$ as a function of layer depth and magnetization. 


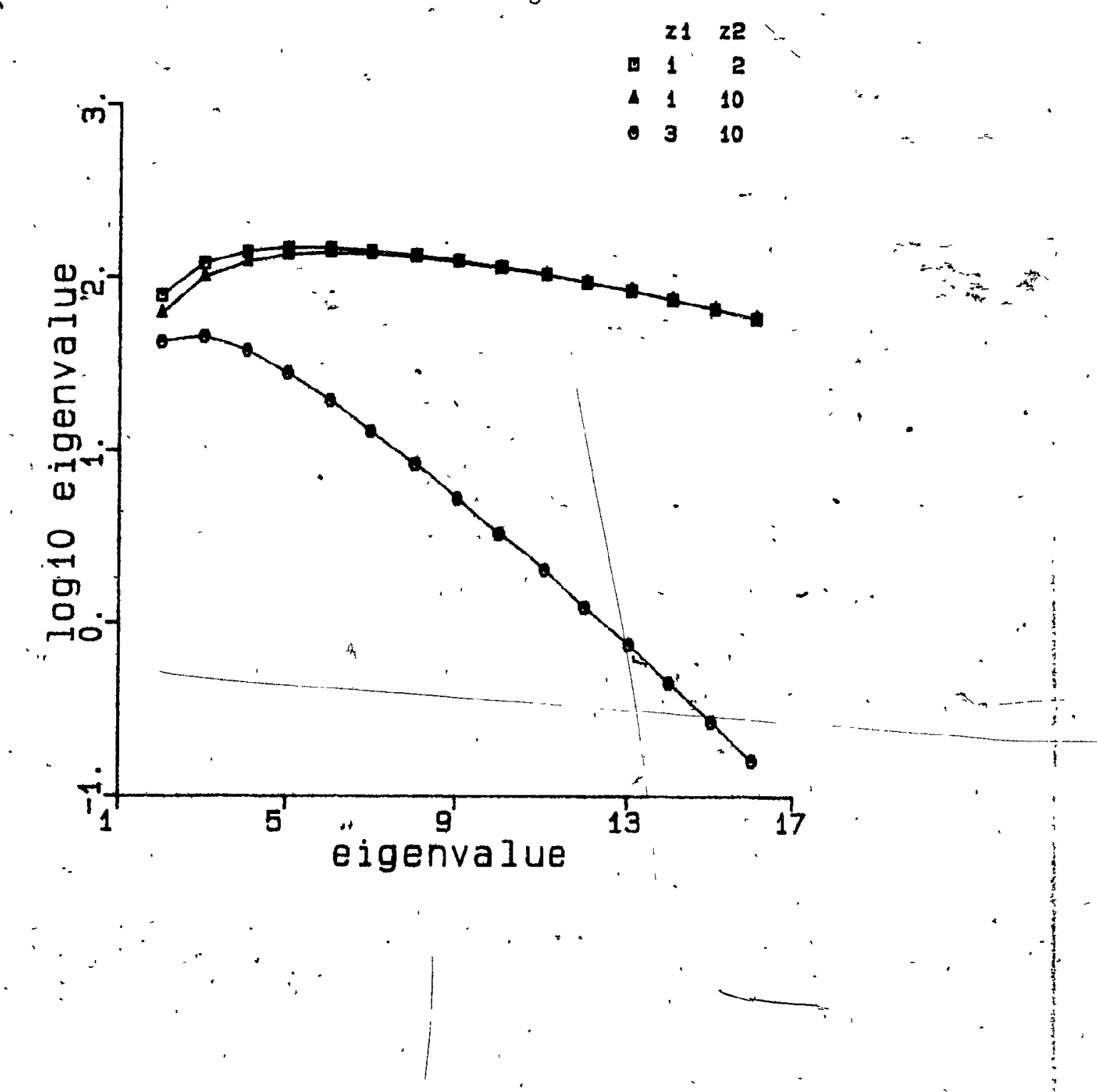

Figure 2.8 Eigenvalue spectra for several two layer cases where $z I$ and $z 2$ give the layer depths in arbitrary units. The value $V\left(F^{2}+G^{2}\right)$ of $(2.7 .2 .7)$ is plotted. Magnetization is $0.6 \mathrm{~A} / \mathrm{m}$. for top layer and $1.2 \mathrm{~A} / \mathrm{m}$ for bottom. 


\section{Table 2.1}

\section{Condition number of the Jacobian for a two} layer model with layer 2 at constant depth.

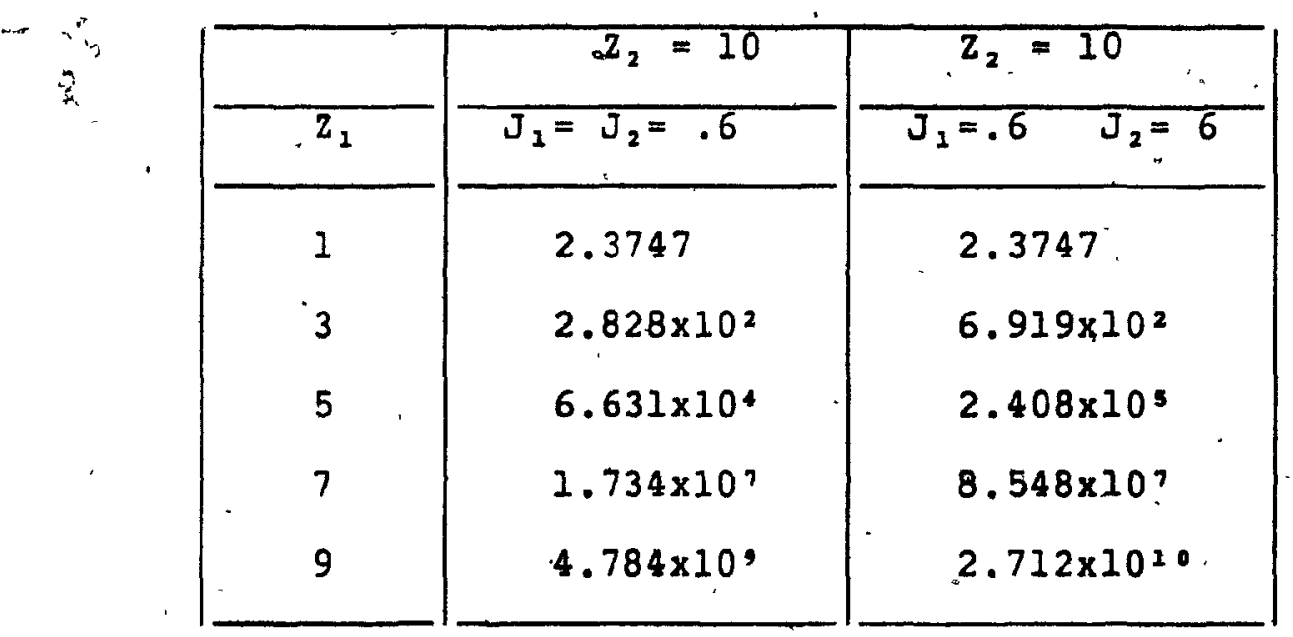

In Tables 2.1 and $2.2, z_{1}, J_{1}$ and $z_{2}, J_{, 2}$ are the depths (arbitrary units) and magnetizations (in $A / m$ ) of the top and bottom layers. The condition number is found from the ratio of the maximum to minimum eigenvalues. In Table 2.1 the sensitivity of the conditioning to the top layer depth is demonstrated while Table 2.2 shows the minor effect of the bottom layer. 
Table 2.2

Condition number of the sacobian for a two layer model with layerl at constant depth.

\begin{tabular}{|c|c|c|}
\hline$Z_{2}$ & $\mathrm{Z}_{1}=1$ \\
\hline 10 & $\mathrm{~J}_{1}=J_{2}=.6$ \\
\hline 8 & 2.3747 \\
6 & 2.3747 \\
2.3748 & 2.3747 \\
2.3781 & 2.8336 \\
2 & 2.0395 \\
\hline$J_{1}=.6 \quad J_{2}=6$ \\
\hline
\end{tabular}

\section{2.}

\subsubsection{Introduction}

The linear equality constrained least squares problem ISE arises when a subset of parameters of the unknown function is required to satisfy specified equality constraints. For the topography problem, information concerning the depths of interfaces may be available from other sources, for example well-log data. Whatever the source, the ambiguity of the problem is effectively reduced by the addition of the constraints. Depending on the dimensionality of the 
prablem, the extra information.may force the solution to the true one.

Problem LSE is posed as: given the linear equation $A x=y$ where $A$ is an nxm matrix, $y$ is an $n$-vector, and $x$ is an $m$-vector, find among all vectors $x$ that satisfy

$B x=C$

exactly, where $B$ is an sxm matrix, and $C$ is an s-vector, the one that minimises

$$
|| A x-y \|^{2}
$$

Obviously the solution exists only if system (2.8.1.1) is consistent. Problem TI will be posed such that (2.8.1.1) has a solution, so the non-uniqueness of the parameter estimates will be focussed onto the system (2.8.1.2):

The imposition of $s$ linearly independent equality constraints reduces the n-dimensional problem to an n-s dimensional one. A solution $x$. can be written in terms of the rów space of $B$ and its orthogonal complement as

$$
x=R x_{s}+N x_{n-s}
$$

where $R$ is $n \times s$ and $N$ is $n x(n-s)$, forming a basis for the row spacf and the null space of $B$, respectively. The s-vector is uniquely determined (Gill et, al.,1981), and any solution satisfies the constraints exactly. It then suffices to specify the $n-s$ vector $x_{n-s}$ for a solution that satisfies 
$A x=y$ in a least squares sense. The constrained inversion is then equivalent to an unconstrained inversion in the remaining $n-s$ parameters. The Lagrange multiplier approach used below provides an explicit partioning of the solution into row and nuli space components.

The simplest method of solving LSE is by direct elimination methods carried out on the augmented system

$$
\left[\begin{array}{l}
A \\
B^{\prime}
\end{array}\right],[x]=\left[\begin{array}{l}
y \\
c
\end{array}\right]
$$

Uniqueness of the solution is guaranteed when the rank of the augmented matrix $A$ is $n$ (Lawson and Hanson, 1974, p.114). A second method is to weight heavily the constraint equations: with respect to the system (2.8.2.2): This leads to a system of augmented normal equations which are soluble in the usual way.

Unfortunately, the above approaches bejcome costly due to the usuaf fequirement of elimination methods for the order of $n^{3}$ computations, Additionally, when the systems are ill-posed, they are better replaced by more stable techniques such as those employing the singular value decomposition. The most straightforward approach is to delete those columns in the Jacobian which correspond to the constrained parameters and perform the SVD on the resulting ... matrix (e.g. Cooke and Schneider, 1983). However the simple form of the decomposition (equation $(2.7 .2 .7)$ ) for the unconstrained case is lost, and it must be determined. 
numerically.

In order to take advantage of the stability of the SVD and to keep computation costs to a minimum, problem ISE is solved using a Lagrange multiplier technique. Initially, the solution is expressed in terms of the Jacobian, $A$, then the known decomposition, equation (2.7.2.7), for $A$ is used to extract a simple computational form for the resulting equations.

\subsubsection{One-layer case}

As developed in section (2.6), the one-layer TI problem estimated $n$ parameters from $n$ observations by a least squares approach. This is necessary when the rank of $A$ (2.8.1.2) is less than $n$, a property, that all magnetic inverse problems have. For this reason, the one-layer constrained inversion, is posed as an overconstrained problem. This is, of course, purely an artificial imposition bearing in mind the remarks on the underdetermined nature of the general inverse problem (section (2.1)).

Given the topography inverse problem,

$$
A x=y
$$

where $x$ are topography values, $y$ are data, and $A$ the partial derivative matrix, a set of $s$ linearly independent equality constraints of the form

$$
\text { , } B \quad x=c
$$


are introduced, with $B$ a known sxn matrix and $c$, a known s-vector of constraint values. The matrix B consists of zeros on each row except a one at the position corresponding to the constrained parameter. This ensures the consistency of system $(2.8 .2 .2)$. With this form of $B$, constraining combinations of parameters is not allowed.

Solving $(2.8 .2 .1)$ in a least squares sense, subject to (2.8.2.2) being satisfied exactly, leads to the following quadratic function to be minimsed:

$$
(y-A x)^{t}(y-A x)+\theta x^{t} x+w^{t}(B x-c)
$$

$\theta$ has been introduced to minimise parameter vector length and stabilise the inversion. $w$ is an n-vector of Lagrange multipliers. Differentiating (2.8.2.3) with respect to $x$ gives the solution

$$
x=\left(A^{t} A+\theta I\right)^{-1}\left(A^{t} y-B^{t} W\right)
$$

where

$$
w=\left(P B^{t}\right)^{-1}\left(P A^{t} y-c\right)
$$

and

$$
P=B\left(A^{t} A+\theta I\right)^{-I}
$$

This is equivalent, for the $\theta=0$ case, to C'rossley and Reid's (1982) formulation. When data and parameter covariances are taken into account (2.8.2.4) generalises to Gubbins's (1984) result (equation (12)). If data and constraints are combined, $(2.8 .2 .4)$ becomes 


$$
X=\left[\begin{array}{ll}
H & H
\end{array}\right]\left[\begin{array}{l}
y \\
C
\end{array}\right]
$$

with

$H_{a}=\left(A^{t} A+\theta I\right)^{-1}\left[I-I^{t}\left[B\left(A^{t} A+\theta I\right)^{-1} B^{t}\right]^{-1} B\left(A^{t} A+\theta I\right)^{-1}\right] A^{t}$

and

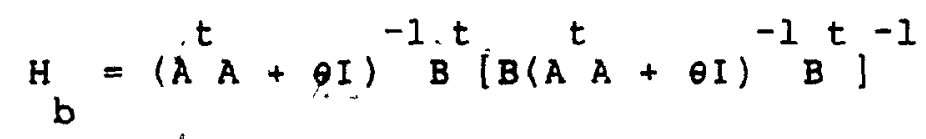

Both $(2.8 .2 .5)$ and $(2.8 .2 .6)$ require the inverse of the mațrix

$$
L=B\left(A^{t} A+\theta I\right)^{-1} B^{t}
$$

This is possible because $L$ is non-singular owing to the positive-definiteness of $\left(A^{t} A+\theta I\right)^{-1}$, which can be written as S S and so $L^{--}=B\left(S^{t} S\right)^{-1} B^{t}=\left(B S^{-1}\right)\left(B S^{-1}\right)^{t}$. The rank of $L$ is then equal to that of $\left(B^{-1}\right)=\operatorname{rank}(B)$ (Graybill, 1983, p.172). Since there are 5 constraints, $\operatorname{rank}(B)=s$. Fortunately, L is of dimensions sxs, which for most problems will be small compared to $n$, so computational efficiency will not be hindered by. inverting $L$. In practice, the Cholesky decomposition is used to find $L^{-1}$.

The inversion of $\left(A^{k} A+\theta I\right)$ is carried out, as in the unconstrained case, using the singular value decomposition. of $A$. When substituted into $(2.8 .2 .5)$ and $(2.8 .2 .6)$ the solution is in the simple form of FFT's and IFFT's amenable ... to straightforward implementation.

Equation $(2.8 .2 .5)$ can be written in the form 


$$
H_{a}=(I-Z) H_{1 s q}=T_{1 s q}^{H}
$$

where $Z=\left(A^{t} A+\theta I\right)^{-1} B^{t}\left(B\left(A^{t} A+\theta I\right)^{-1} B^{t}\right)^{-1} B$ and $H_{1 s q}$ is the unconstrained least squares inverse. It is easy to show that the operators $T$ and $Z$ have the following properties:

$$
T Z=Z T=0 ; \quad Z^{2}=Z ; T^{2}=T
$$
-

and, if the a posteriori parameter covariance for the unconstrained case is $c_{\text {loq }}(2.5 .2 .13)$, then

$$
T C_{1 s q}=C_{1 s q} T^{t} \quad z C_{1 s q}=C_{1 s q} z^{t}
$$

Equations $(2.8 .2 .8)$ and $(2.8 .2 .9)$ imply that $z$ and $T$ are projection matrices (Strang, 1980, p.132) orthogonal relative to $\mathrm{C}_{19 q}$. If $\mathrm{C}_{\mathrm{con}}$ is the constrained covariance, a simple relation exists between this quantity and its unconstrained counterpart:

$$
\mathrm{C}_{\text {con }}=\mathrm{TC}_{\text {lsq }}
$$

It should also be noted that ' $B T=0$, which shows the projection by $T$ is orthogonal to the constrain plane in parameter space.

By analogy with the unconstrained case, the resolution matrix for the constrained problem is given by 


$$
\mathbb{C o n}_{\mathbf{a}}=\underset{\mathbf{b}^{-}}{\mathrm{H}}
$$

From (2.8.2.7) Rcon can be.written as

$$
R_{\text {con }}=R_{1 s q}+z\left(I-R_{1 s q}\right)
$$

with $R$ leq being the unconstrained resolution matrix and $I$ has dimensions mxm. Examination of the structure of $z$ reveals that rows corresponding to constrained parameters, say row $i$, consist of zeros except for a one at column i. Considering the ith row of $R_{\text {can }}\left(r_{r c o n}^{i}\right)$

$$
\begin{aligned}
r^{i} \text { con } & =r^{i}+z^{i}\left(I-k_{l s q}\right) \\
& =z^{i} \\
& =e^{i}
\end{aligned}
$$

where $e^{i}$, is the ith row of the identity matrix. This guarantees that each constrained parameter is perfectly resolved. Similarly, from (2.8.2.11), if an unconstrained parameter is perfectly resolved by the data, this situation is. unchanged by the addition of the constraints.

\subsubsection{Two-layer case}

The two-layer case as posed here, is underdetermined, therefore the theory of section $(2: 8.2)$ is innapplicable due to the singularity of the matrix $A^{t} A$. Constrained inversion for the underdetermined problem has been studied by Burkhard. 
and Jackson (1976), who solve the problem using a Lagrange $S$ multiplier approach. In the two-layer case, $m>n$; for two layers $m=2 n$. It is necessary to introduce some form of constraint to reduce the non-uniqueness of the estimated parameters. This can be done by minimising parameter vector length as usual, but introducing constraints of the form $B x=c$ Ento the solution. The quadratic form to be minimized is then

$$
x^{t} x+\left(x^{t} A^{t}-y^{t}\right) w+\left(x^{t} B^{t}-c^{t}\right)
$$

where $w$ and $n$ are Lagrange multiplier vectors. Differentiating, the solution is of the form

$$
x=A^{t} w+B^{t} n
$$

Assuming the constraints are satisfied exactly,

$$
B x=c=B A^{t} w+B B^{t} n
$$

The multiplier $n$ is then given by

$$
n=\left(B B^{t}\right)^{-1}\left(B A^{t} w-c\right)
$$

Substituting in $(2.8 .3 .2)$ gives

$$
x=B^{t}\left(B B^{t}\right)^{-1} c+\left[I-B^{t}\left(B B^{t}\right)^{-1} B\right] A^{t} w
$$

Introducing $\theta$, as in the one-layer case, to stabilise the 
inversion, a combination of residuals and "parameter vector length can be minimized:

$$
\min (y-A x)^{t}(y-A x)+\theta x^{t} x
$$

Differentiating with respect to w and using the expression $(2.8 .3 .3)$ for $x$ gives

$$
A Q A A Q A w-A Q A Y+A Q{ }^{t}{ }^{t}{ }^{t} A P C+\theta A Q Q A A^{t} w+\theta A Q P C=0
$$

where $P=B^{t}\left(B^{t}\right)^{-1}$, and $Q=I-P B$. Since $Q^{t} Q=Q$, and $Q^{t} P=$ 0 , this reduces to',

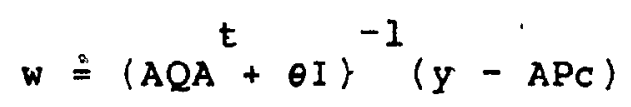

and

$$
x=Q A^{t} \cdot\left(A Q A^{t}+\theta I\right)^{-1}(y-A P C)+P C
$$

Partitioning $(2 \cdot 8 \cdot 3.5)$ into data and constraint parts,

$$
x=\left[\begin{array}{ll}
H_{a} & H_{b}
\end{array}\right]\left[\begin{array}{l}
y \\
c
\end{array}\right]
$$

with

and

$$
H=Q A A^{t}\left(A Q A^{t}+\theta I\right)^{-1}
$$

$$
\mathrm{H}_{\mathrm{b}}=\left(\begin{array}{cc}
\mathrm{I} & -\mathrm{H} A
\end{array}\right) \mathrm{P}
$$

Contrary to the one-layer case where an inverse of only sxs was required, the matrix $M=\left(A Q A^{t}+\theta I\right)$ is td be inverted, of dimensions nxn. For map size data, this is 
computationally prohibitive to perform explicitly. If $B$ has the form described in section (2.8.2), then $Q$ is of rank n-s and $A Q A$ t is at most rank $n-s$. The damping or trade-off factor $\theta$ is therefore essential for a stable inversion. M maybe written

$$
M=\left(A A^{t}-A B^{t} B A^{t}+\theta I\right)
$$

from the definition-of $Q$. The SVD. of $A$ is known (equation $2.7 .2 .7)$ so that

$$
A A^{t}=E^{\star}\left(F^{2}+G^{2}\right) E
$$

The addition of $\theta I$ does not change the circulant-character of $A A^{t}$, however the subtraction of $A B^{t} B A^{t}$ (rank $s$ ) renders $M$ non-circulant. No simple factorization exists for $M$ (Gill and Murray, 2977). If the SVD of matrix AA is updated as a result of the subtracted term in (2.8.3.7) this would require of the order of $\mathrm{n}^{3}$ computations (Businger, 1970).

A simple iterative procedure will be outlined for the stable inversion of $\mathrm{M}$ in which the known decomposition for $\mathrm{A}$ is exploited for numerical efficiency. First, splitting $M$ into circulant and non-circulant parts:

$$
\begin{aligned}
M & =\left(A A^{t}+\theta I\right)-A B^{t} A^{t} \\
& =-T^{\prime}-S
\end{aligned}
$$

From (2.8.3.6), the contribution to the solution from the 
data is

$$
\begin{aligned}
x & =Q A^{t}\left(A Q A^{t}+\theta I\right)^{-1} y \\
& =Q A^{t} z
\end{aligned}
$$

with

$$
z=M^{-1} y
$$

A recursive procedure can be implemented for solving $(2.8 \cdot 3.10):$

$$
\begin{array}{ll}
z_{k+1} & =z_{k}+T^{-1}\left(y-M_{k}\right) \\
\text { or } z_{k+1} & =\left(I-T^{-1} M\right) z_{k}+T^{-1} y
\end{array}
$$

This fixed point iterative scheme starting from any $z$ converges to the unique solution $\zeta$ of $\left(\mathrm{AQA}^{t}+\theta \mathrm{I}\right) \zeta=y$, if ||$I-T^{-1} M|| \leq I$ for some matrix norm (Conte and deBoor, 1980, p.225). The error at each step is reduced by at least a factor of $K=|| I-T^{-1} M||$. Writing $K$ in terms of its constituent matrices,

$$
\begin{aligned}
I-T^{-1} M & =I-\left(A A^{t}+\theta I\right)^{-1}\left(A A^{t}+\theta I-A B^{t} B A^{t}\right) \\
& =\left(A A^{t}+\theta I\right)^{-1}\left(A B_{B A}^{t}\right)
\end{aligned}
$$

Now if $S=A B^{t} B A^{t}$ and $R=A A^{t}$, then 


$$
S=\sum_{i=1}^{s} a_{i} a_{i}^{t} ; \quad R=\sum_{i=I}^{m} a_{i} a_{i}^{t}
$$

The norm of each outer product $a_{1} a_{1}^{t}$ is constant over $i$ because each $a_{i}$ can be written in terms of any other $a_{j}$ by $a_{i}=P^{i j} a_{j}$, where $P^{i j}$. is a permutation matrix of norm 1 . Because the number of outer products is less in $S$, then ||$s||<|| R||$. so

$$
\begin{aligned}
K & =\left\|\left(A A^{t}+\theta I\right)^{-I}\right\|\|\|^{t} B A^{t} \| \\
& <\left\|\left(A A^{t}+\theta I\right)^{-I}\right\|\left\|A^{t}\right\|
\end{aligned}
$$

In order to show that $k<1$, the spectral radius is used, where it is known that for any norm of the matrix $A,|| A||<$ $\rho(A)$, with $\rho($.$) denoting spectral radius. \rho(A)$. is equal to. the maximum eigenvalue of $A$, so from $(2.8 .3 .12)$

$$
\begin{aligned}
K & <\left(A A^{t}+\theta I\right)^{-1} \cdot\left(A A^{t}\right) \\
& <\frac{1}{\max \left(f^{2}+g^{2}+\theta\right)} \cdot \max \left(f^{2} \pm g^{2}\right) \\
& <1
\end{aligned}
$$

where (2.8.3.14) follows from the SVD's of the corresponding terms in (2.8.3.13). The limit for $K$ is an upper bound. The factors controlling its magnitude are $\theta$, the damping factor and $s$, the number of constraints. Increasing the damping, or decreasing the number of constrained parameters will result. . 
in improved convergence.

Once the iterative scheme of $(2.8 .3 .11)$ gives $z$, this can be substituted into $(2: 8.3 .10)$ to find the contribution to the topography from the data. Equation (2.8.3.11) is then used in a similar fashion to find the contribution from the constraints $\left(x_{b}\right)$ by making the substitutions

$$
\begin{aligned}
& z=B^{t}\left(B B^{t}\right)^{-1} c-x_{b} \\
& y=A B^{t}\left(B B^{t}\right)^{-1} c
\end{aligned}
$$

As the constraint system $B x=C$ is linear, oniy one iteratipin is required for the determination of $x_{b}$.

The computational labour is further reduced in the case of a single constraint since a simple non-iterative expression can be derived for the inverse of the matrix $M$ in $(2.8 .3 .7)$. The presence of constraints can be treated as a rank one perturbation to the matrix $\left(A A^{t}+\theta I\right)$, that is, $A B^{t} A^{t}=a_{c} a_{c}^{k}$, where $a_{c}$ is the column of $A$ corresponding to the constrained parameter. The subsequent change to the inverse of $A^{\text {t }}$ (dropping, the damping factor) is given by (e.g. Graybill, 1983, p.231)

$$
\begin{aligned}
& \left(A A_{1}\right)^{t-1}=\left(A A^{t}\right)^{-1}-\left(A A^{t}\right)^{-1} a_{c} a_{c}\left(A A^{t}\right)^{-1} \\
& a_{c}\left(A A^{E}\right)^{-1} a_{c}^{E}-1
\end{aligned}
$$

if $A A_{1}^{t}=A A^{t}-a_{c} a_{c}^{t}$, and providing the denominator is not zero. The solution for the problem LSE can also be stated in. 
terms of generalized or pseudo-inverses (Albert, 1972, p.31) as

$$
x=B^{+} c+\left(A\left[I-B^{+} B\right]\right)^{+}\left(y-A^{+} c\right)
$$

where + denotes generalized inverse. The two terms in (2.8.3.16) split the solution into row and null space portions of $B$. Substituting $B^{t}\left(B B^{k}\right)^{-1}$ for $B^{+}$, and the similar form for $\left(A\left(I-B^{+} B\right)\right)^{+}$, the solution of $(2.8 .3 .5)$ is arrived at. Now it is known that the general solution of $A x$ $=y$ is

$$
x=A^{+} y+\left(I-A^{+} A\right) t
$$

t being an arbitrary vector of length $m$. It follows that the general solution for the constrained problem is then $\tilde{x}=\mathrm{B}^{+} \mathrm{C}$ plus a vector $h$ that minimises ||$A x-y||^{2}$. h is found to be (Graybill, 1983, p.173)

$$
\begin{aligned}
\dot{h} & =\left(I-B^{+} B\right)\left[I-\left(A\left(I-B^{+} B\right)\right)^{+}\left(A\left(I-B^{+} B\right)\right)\right] z \\
& =\left(Q-Q{ }^{t}(A Q A)^{t} A Q\right) z
\end{aligned}
$$

with $z$ being an arbitrary m-vector, and using $B^{+}=B^{t}$. Thus ${ }^{*}=$ (2.8.3.18) summarises the non-uniqueness of the constructed solution $(2.8 .3 .16)$. It is interesting to note that this solution is also the one that minimises $\|A x-y\|^{2}$ and $\|\mathrm{Bx}-\mathrm{C}\|^{2}$ simultaneously. 


\section{CHAPTER $\underline{3}$}

\section{$\therefore \quad \underline{3} . \underline{1}$ Introduction}

In this chapter practical examples of the method developed in section: $(2.6)$ are presented. Simple trial and error procedures are introduced for the determination of auxiliary parameters such as interface depth and magnetization. The stability of the algorithm is investigated under the conditions of poorly.chosen starting models and noise. Comparisons are made with a fully nonlineär approach using numerically determined SVD's at each iteration. The effect of relaxing some of the a priori assupptions concerning the model is . investigated with emphasis on stability and computational labour.

3.2 Determination of auxiliary parameters

3.2.1 Damping factor

In section $(2.6 .3)^{\circ}$. it was mentioned that the damping factor or Marquardt parameter performs a filtering of the 
observations. Without considering the presence of data errors, the downward continuation implicit in the method requires rather severe filtering. From the eigenvalue spectrum of the problem this affects the high wavenumber components causing a smoothing of the data. Fortunately, this takes care of spurious high frequency effects present due to gridding, observational noise and preprocessing such as those arising from the use of the Fast Fourier transform which is notoriously unireliable at the high wavenumber end of the spectrum (e.g Cordell and Grauch, 1982). As well as smoothing the final topography estimates, damping improves the stability of the iterative process by bounding possible parameter corrections. From the known trade-off between resolution and variance (section (2.5)), the damped solution achieves better statistical reliability. Accompanying the - decrease in resolution is the possible increase in misfit between data and. predicted responses due to the neglect of certain high efrequency solution components. The imposition of smoothness constraints is physically reasonable in the case of potential fields arising from interfaces since the observed field is mainly a function of the bulk properties of the source, hence the small scale features are not so important. From the computational viewpoint, reffriction of the solution to the lower wavenumbers allows the algorithm to negate the severely damped wavenumber components. In practical applications the passband can be a small fraction 
of the spectrum of the original data, causing a significant reduction of operations.

Figures 3.2 .1 and 3.2 .2 illustrate the effect of the damping factor on the inverse eigenvalue spectrum. The ratio of damped to undamped inverse eigenvalues is plotted. in Figure 3.2.1 for several values of damping factor for a layer at a depth of 6 units and with a magnetization of 1.2 $A / m$. Since there are only $1+n / 2$ distinct eigenvalues for $n$ data and the zero eigenvalue is removed from the solution, the resulits are shown for the 2 nd to the $(I+n / 2) s t$ eigenvalue. As the damping factor is increased the low-pass filtering effect becomes more pronounced. An example of undamped and damped inverse eigenvalue spectra is given in Figure'3.2.2. It emphasises the severe attenuation of the higher wavenumber components needed to suppress oscillations in the solution estimates: the damped spectrum shown produced an acceptable solution (curve for magnetization 1.2 $\mathrm{A} / \mathrm{m}$ in Figure 3.2.7).

There is no way of knowing, a priori, the amount of filtering required, although it is obvious that some broad relation exists between the highest frequency kept in the solution and the average depth of the interface. Gerard and Debeglia (1975) have suggested a rule to reduce the cut-off frequency from $1 / 2 \mathrm{dx}$ (Nyquist frequency) to $1 / \mathrm{pdx}$, where $\mathrm{dx}$ is the grid interval and $p$ is found from

$$
\mathrm{p} / 2<\mathrm{z}_{0} / \mathrm{dx}
$$


。

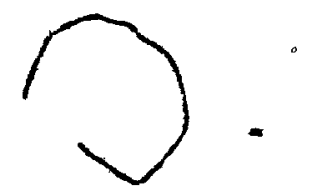

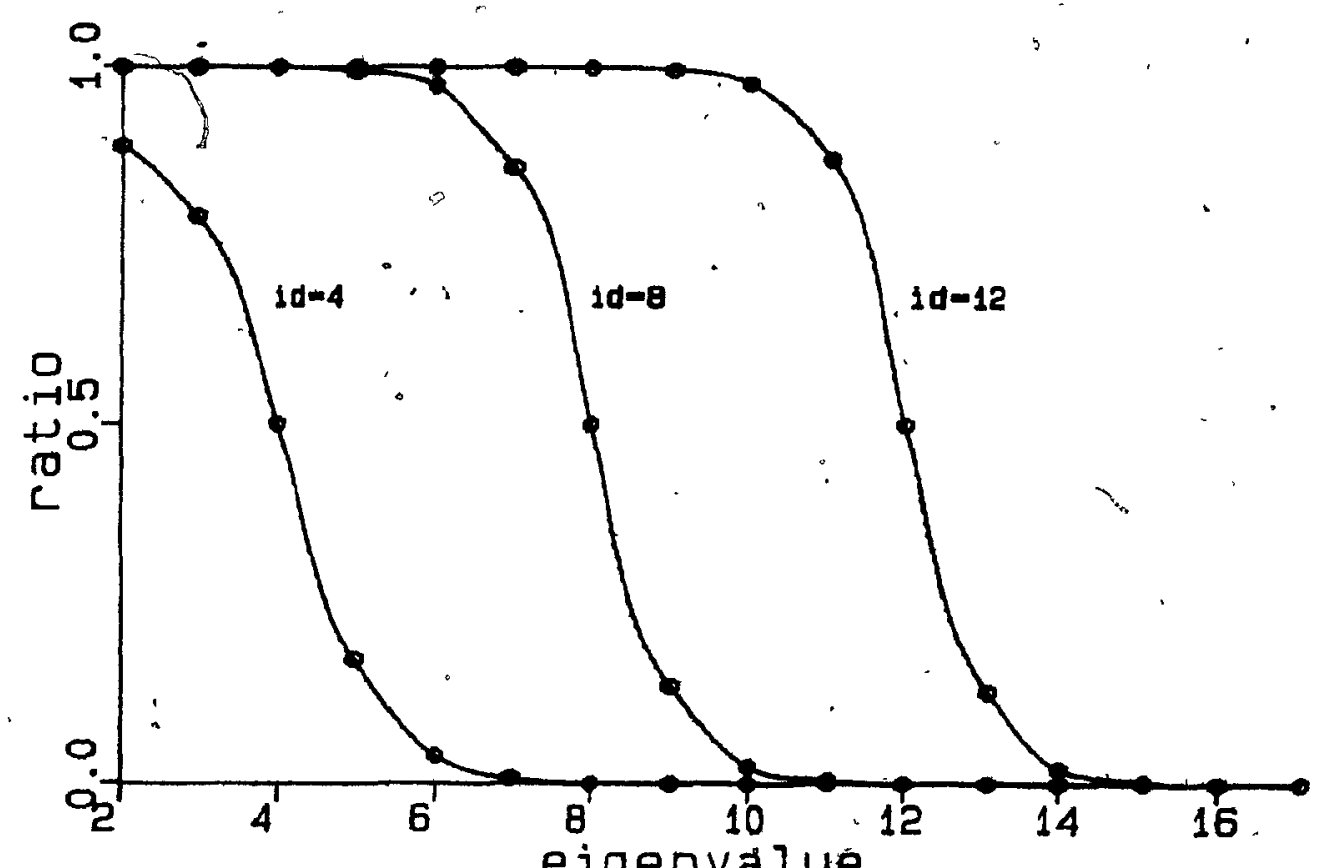

ejgenval lue

$\because$

Figure 3.2.I. Ratio of inverse and damped inverse eigenvalues for a layer at depth 6 units and magnetization $1.2 \mathrm{~A} / \mathrm{m}$. Values of ID indicate the number of eigenvalue used as a damping factor. 


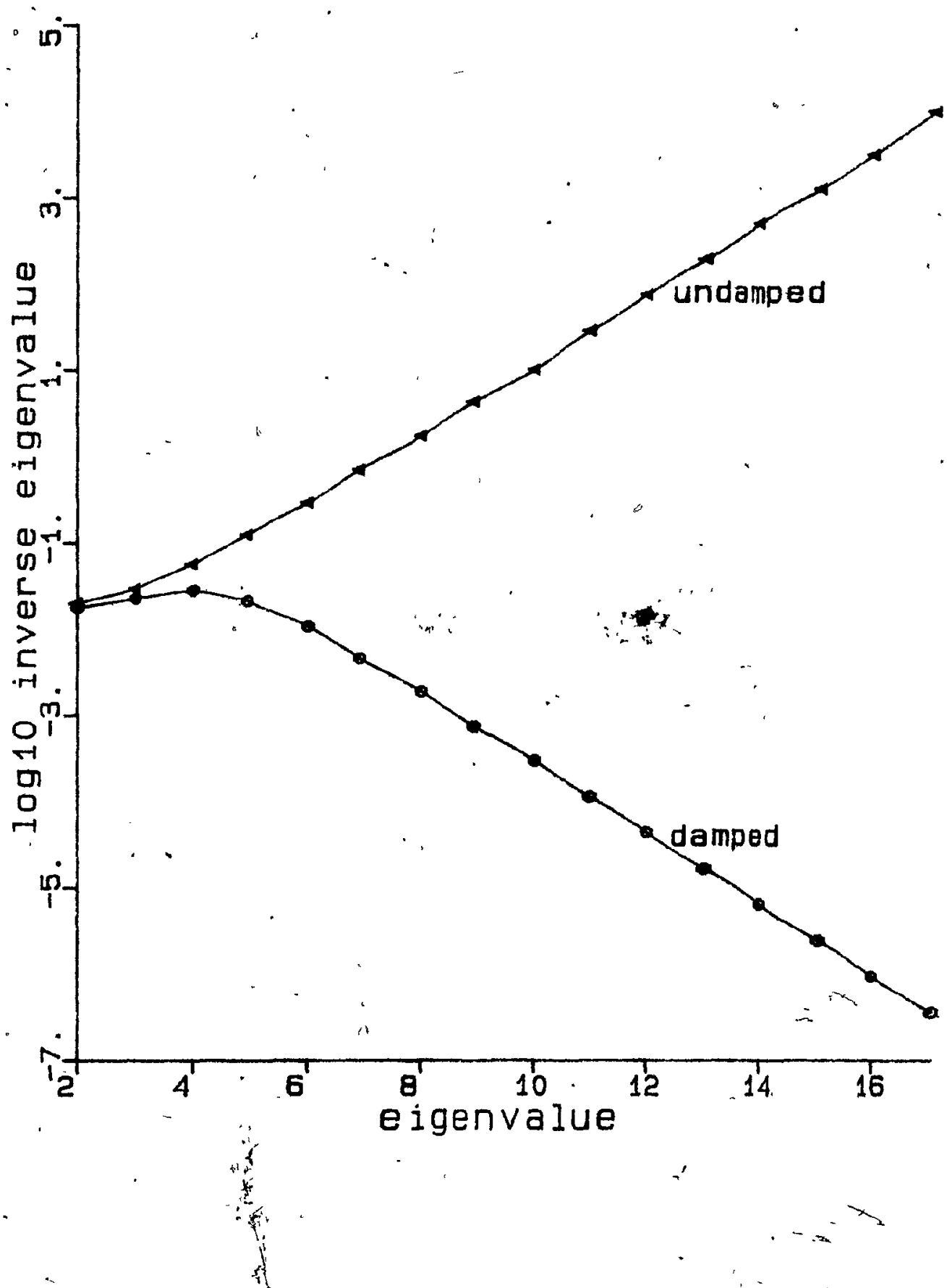

Figure 3.2 .2 Effect of damping on inverse eigenvalue spectrum. Damped spectrum was produced by a damping factor equal to the fourth eigenvalue. 
with $z_{0}$ being the averáge interface depth.

Another more objective approach is to examine directly the inverse eigenvalues and determine an a priori limit on their magnitude based on the expected size of the interface variation. Taking the $\infty$-norm of the first iteration (equation $(2.6 .3 .7)$ )

$$
\|x\| \leq,\left\|A^{+}\right\|\|y\|
$$

where $x$ is the first topography iterate and $y$, is the data. w

Using the spectral radius of $\mathrm{A}^{+}$

$$
\begin{aligned}
|| \hat{x}|| & \leq \rho\left(A^{+}\right)|| y|| \\
& =\max _{i} \lambda_{i}^{-1}|| y||
\end{aligned}
$$

with $\lambda_{i}$ being the eigenvalues given by (2.6.3.4). Since ||$y||$ is known and a reasonable estimate of $\|x\|$ can be found, the ratio $\|x\| /|| y \|$ can be determined. It then suffices to change the inequality in (3.1) to an equality and find the smallest wavenumber that satisfies the relation. Equation (3.1) gives a, lower bound on the maximum inverse eigenvalue, so the resulting wavenumber will be a conservative estimate. Converting the maximum inverse eigenvalue (occuming at say wavenumber $j$ ) into a damping factor is straightforward once the general form of Figure 3.2.I is noted. If the jth eigenvalue is taken as the damping factor this results in a halving of the $j$ th inverse 
eigenvalue. The higher wavenumbers are damped more severely, although if $z_{0}$ is large it may not be severe enough to guarantee the inequality (3.1). However, this does not. present any difficulty as the choice of $j$ is a conservative one. In all inversions attempted, this method has been successful when ||$x||$ is well estimated.

As an illustration of this approach, consider model L2 (Figure 3.2.3). Table 3.1 shows the maximum damped inverse eigenvalue as a function of damping factor (chosen to be successive eigenvalues).

Table 3.1

Maximum damped inverse eigenvalue as a function of damping factor

Damping factor $\quad 50.36 \quad 34.37 \quad 17.65 \quad 8.08 \quad 3.48$ $\begin{array}{lllllll}\operatorname{Max} & \lambda_{0}^{-1} & .0099 & .0145 & .0283 & .0618 & .1437\end{array}$

From the observed data (Figure 3.2.3), ||$y||=106$. Setting an esimate for the maximum expected variation in topography, ||$x||=2.5$, then

$$
|| x|| /|| y||=0.02358
$$

suggesting a damping factor at about the fourth eigenvalue (17.65). As will be shown later, this choice resulted in the most rapid convergence (see Table 3.3 ). 

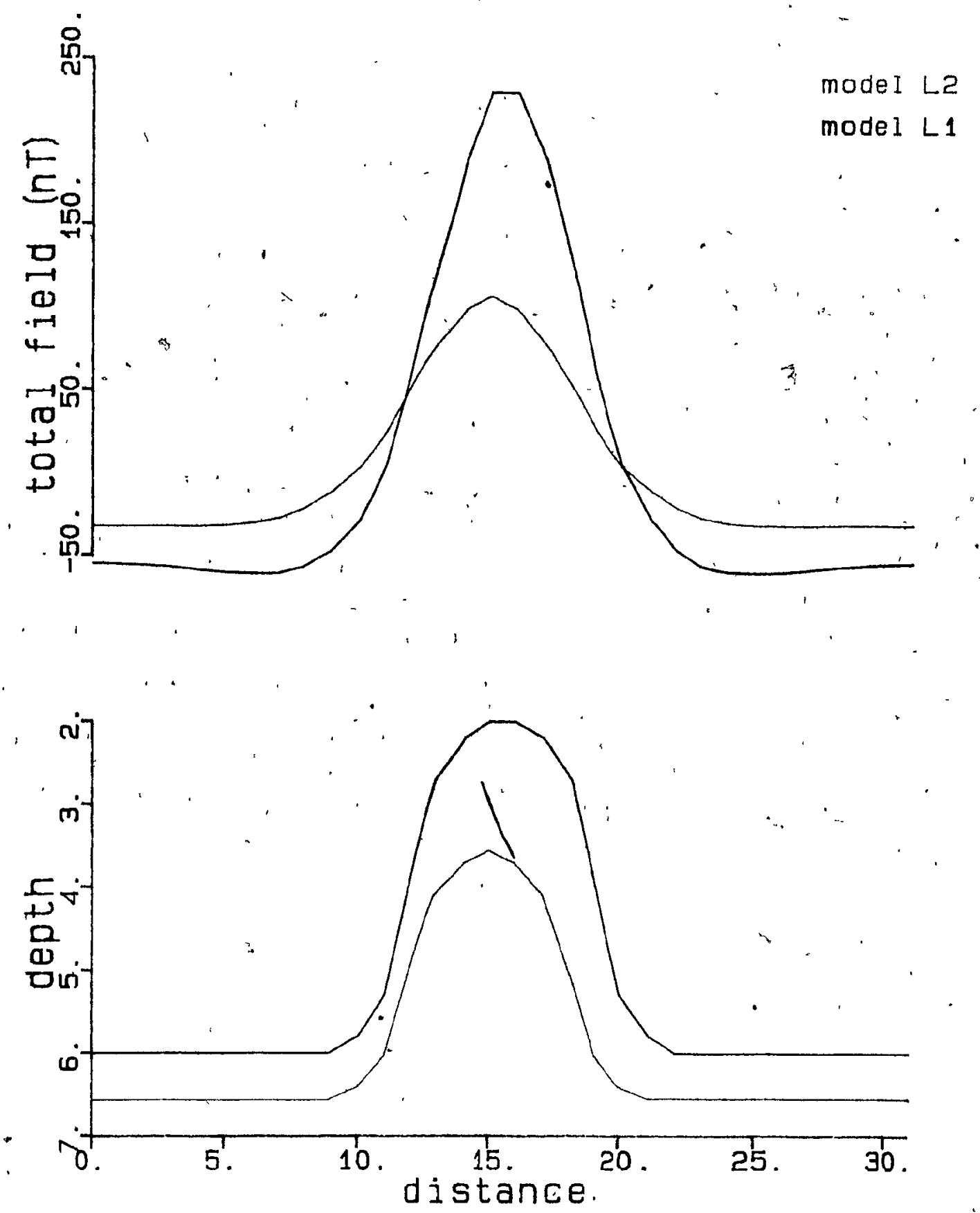

Figure 3.2.3 Model LI topography and calculated ef fect. Average layer depth is 5.26 arbitrary units with a magnetization of $1.2 \mathrm{~A} / \mathrm{m}$. Model $\mathrm{L} 2$ topography and calculated effect. Average layer depth is 6 arbitrary units. with a magnetization of $1.2 \mathrm{~A} / \mathrm{m}$. 
The damping factor is the most important auxiliary parameter because of its direct influence on the convergence of the algorithm. It is not $a$,unique choice and can be varied to produce solutions with varying frequency content. Increasing the damping factor causes smoother topographies and the possibility of a poorer fit to the data. The sensitivity of the algorithm's convergence on damping is illustrated in Figure 3.2.4 where model L2 data has been inverted with damping factors ranging from 5 t'o 50 . Values below 5 produced divergence. Unfortunately the value of $\theta$ (damping factor) is problem dependent so it is aifficuit to relate from one problem to another.

\subsubsection{Depth and magnetization}

A particular form of ambiguity is inherent to models consisting of two differently magnetized media separated by a variable interface: namely the indistinguishable nature of the average interface depth $\left(z_{0}\right)$ and its magnetization ( $\left.J\right)^{\text {.' }}$ A variety of $\left(z_{0}, J\right)$ pairs can be found that satisfy the data (with a corresponding adjustment of the topography). Figure 3.2 .5 exemplifies the problems to be faced. Shown is the objective function (RMS error) space as a function of $z_{0}$ and $J$ while all other parameters are held constant for the synthetic data of model Ll (Figure 3.2.3). Even with this constraint, an elongated trough of acceptable $z_{0}, J$ pairs exists. This shows a direct relation, between $z_{0}$ and $J$ for 


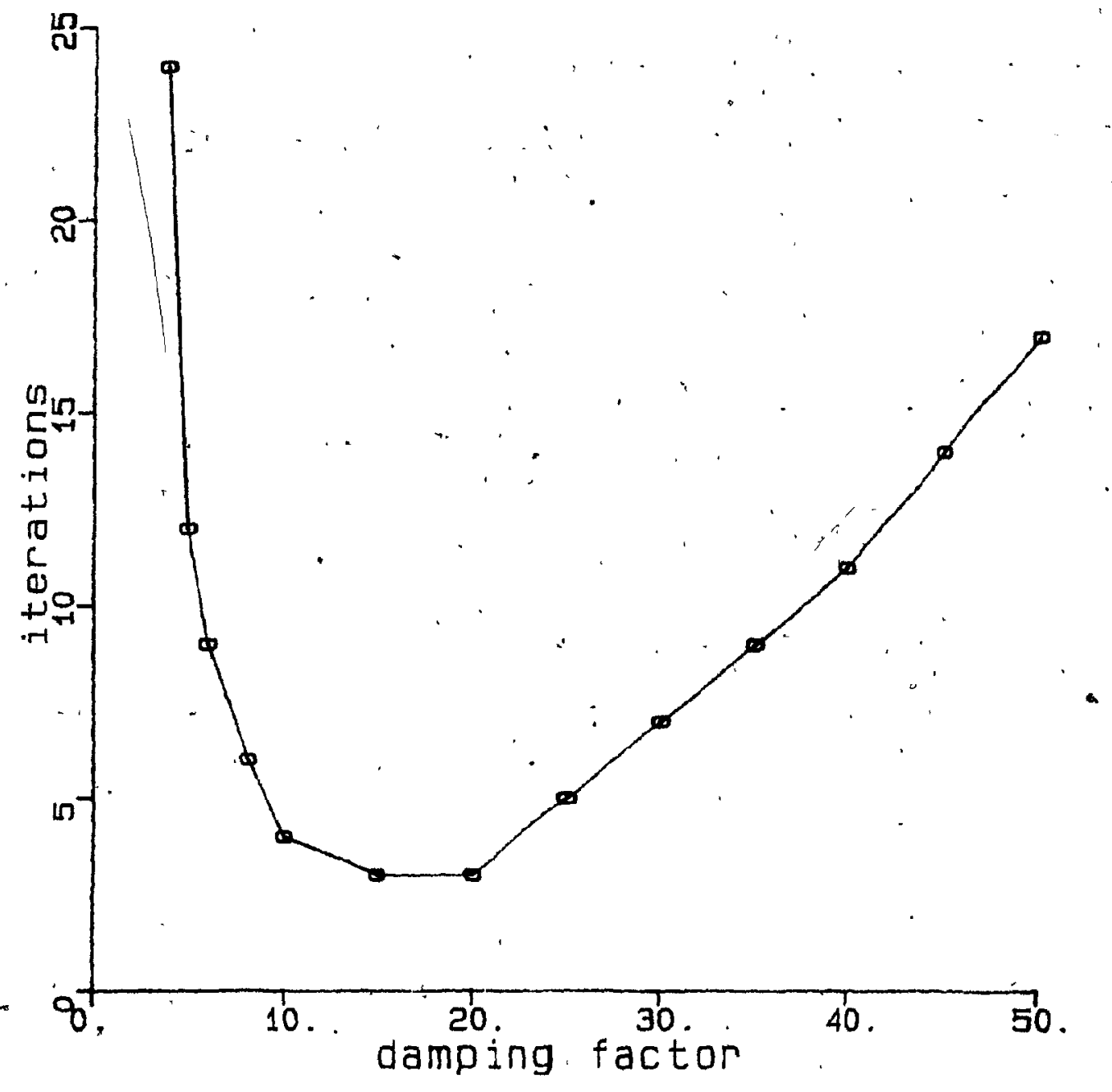

Figure 3.2.4 Effect of damping on number of iterations required to reduce misfit to less than $1 \mathrm{nT}^{2}$ for model L2. 


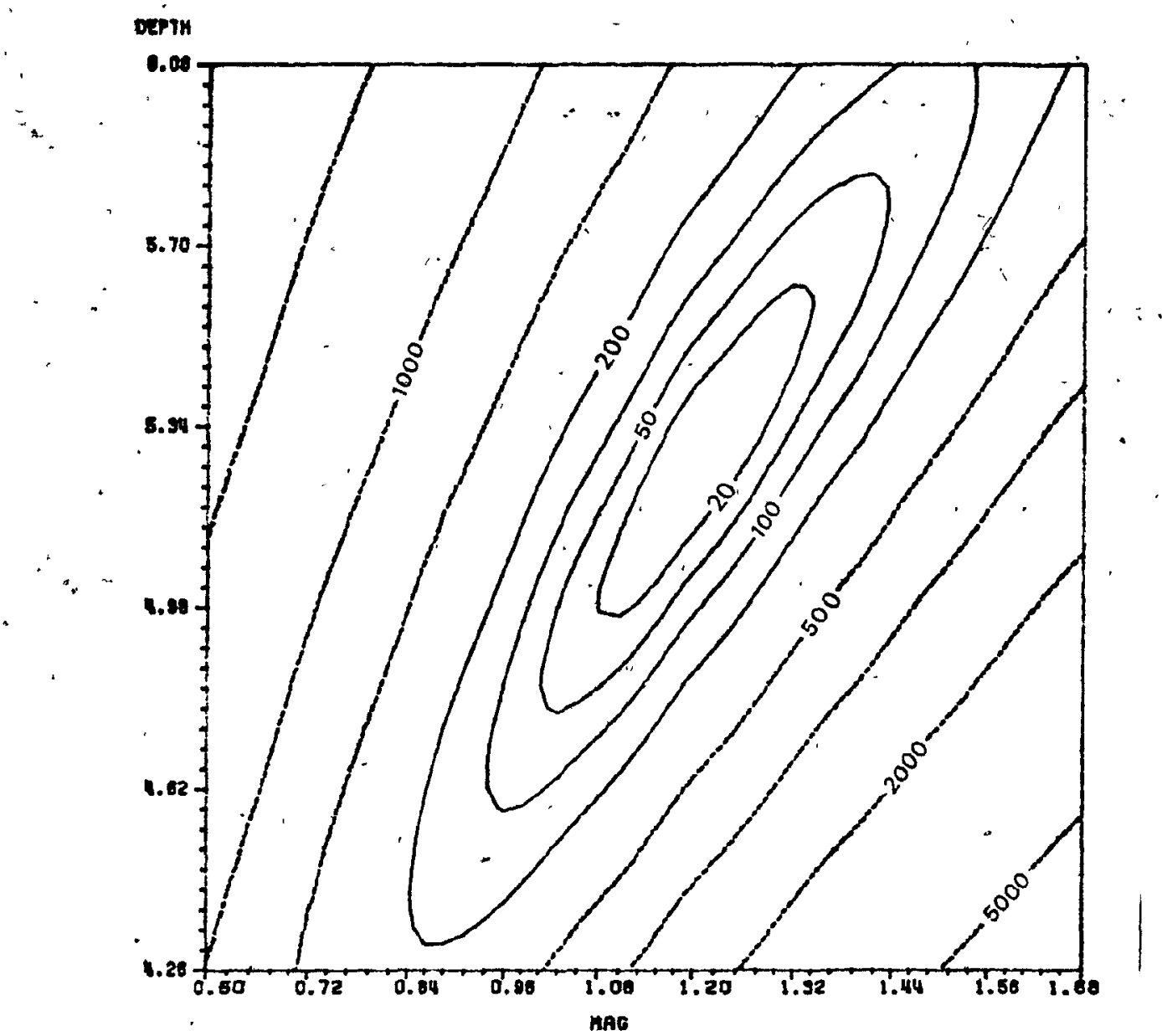

Figure 3.2.5 Contour map of RMS error for model $\mathrm{Ll}$.

19 
models satisfying the observations. It is therefore paramount to have independent knowledge about one or both of these parameters. In the absense of geologic or arilling control the easier parameter to determine is the average depth. In this regard spectral methods are relevant.

The basis of the spectral approach is the simple relationship between* the spatial frequency content of the potential field anomaly and the depth to its source (Troshkov and Shalaev, 1961; Solovyev, 1962). If certain assumptions concerning the distribution of source parameters are satisfied by the sources, the log-energy spectrum of the observations will be a straight line with a slope proportional to the average source depth (Spector and Grant, 1970). Deriving this relationship requires the specification of a causative model. The most versatile and hence most popular is the vertical prism, although lines of poles (Treitel et al, 1971), and dykes and cylinders (Pedersen, 1978) have also been used. For problem TI, the model is the single interface or layer for which the relationship between energy spectrum and depth is manifest by the form of the forward equation (2.6.2.4).

Taking the first term in the expression for the field due to a constantly magnetized topography gives the first I inear approximate topography (FLAT):

$$
B(\vec{k})=e^{-|\vec{k}| z_{0}} v(\vec{k})|\vec{k}| \mathcal{F}[h(x)]
$$


$\mathrm{V}(\vec{k})$ contains magnetization and geomagnetic factors and the other quantities are those of $(2.6 .2 .4)$. If a simple relationship exists between the average source depth and the spectral properties of the observed field, $F[h(x)]$ should be approximately. constant at long wavelengths. Herè $|k| h(x)_{\text {max }}<<1$ which ensures that the FLAT is a good approximation to the true topography. In this part of the frequency band the spectrum approaches that of a random distribution of dipoles, hence it is not influericed by the structure of $h(x)$. Consequently the, slope of the log-spectrum will allow $z_{0}$ to be determined.

The validity of the FLAT for depth estimation is dependent on the properties of the topography function and how this affects the higher order terms in (3.2); it is not influenced by the average depth itself. Insight into such “behaviour can be gained by considerìng representative topographies with simple mathematical expressions. For example, considering the function

$$
h(x)=\frac{A a^{2}}{x^{2}+a^{2}}
$$

it has amplitude $A$, and a half-width of a. Figure 3.2.6 shows some representative relief'functions of the form above. Fourier transforming the first two terms in the expansion $(2.6 .2 .4)$, 
$\therefore$

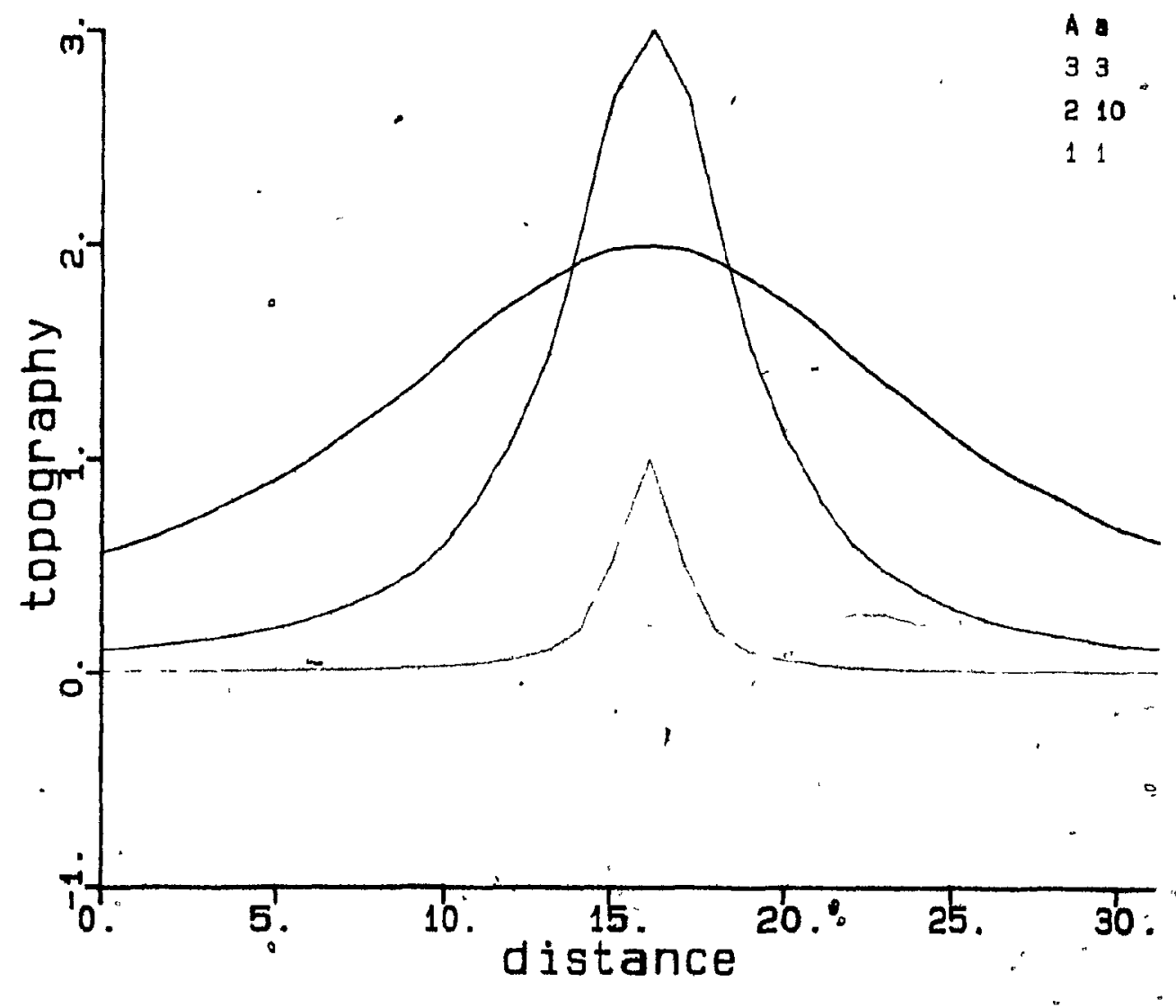

Figure 3.2 .6 Representative topograhies of the form $h(x)=A a^{2} /\left(a^{2}+x^{2}\right)$ where the amplitude $A$ and half-width a are given. 


$$
F[h(x)]=A a e^{-k a}
$$

and

$$
\mathcal{F}\left[h^{2}(x)\right]=\frac{(1+k a) A^{2} a e^{-k a}}{2}
$$

Forming the ratio of irst to second terms

$$
F_{12}=\frac{F\left[h^{2}(x)\right] k}{\mathscr{F}[h(x)] 2}=A k(I+k a) / 4
$$

So for $F_{12}$ to be as smal, as possible, $A, k$ and a shouldalso be small; the topography strouild be of low amplitide and high frequency content for the FLAT to be useful as a depth estimator: Equation (3.3) shows the deleterious effects of including the higher. wavenumbers in spectral determination of interface depth. These results are not in conflict with those of Mishra and Pedersen (1982) who investigated the properties of a Gaussian relief function. Diminished relief wavelength primarily increases the whiteness of the spectrum while the amplitude controls the. influence of the higher order terms in the expansion. Karner and Watts (1983) give an example of the use of the spectral approach to depth estimation from gravity data.

The influence of the statistical nature of the topography is easily seen by treating an uncorrelated relief as an example. If $h(x)$ is white, then $\mathscr{F}[h(r)]=H$ (a cónstant) and $\mathscr{F}\left[h^{2}(x)\right]=H^{2}$. Then from $(3.2)$ 


$$
\begin{aligned}
B(\vec{k}) & =e^{-|\vec{k}| z_{0}} v(\vec{k}) \sum_{n=1}^{\infty} \mid \frac{\vec{k}}{\vec{n} !} H^{n} \\
& =e^{-|\vec{k}| z_{0}} v(\vec{k}) \cdot\left(e^{|\vec{k}| H}-1\right)
\end{aligned}
$$

and a linear relation exists between spectrum and depth. The fluctuations, around the average depth lead to an * underestimation of, $z_{0}$ by an amount equal to the variance $H$ of the topography.

For a given value of $z_{0}$, a variety of magnetizations exist that can be specified to satisfy the observations when the data are inverted. To first order, an inverse relationship is obeyed by the magnitude of the layer. magnetization and its corresponding topography. This was noted by Hahn (1966) who used the FLAT in his harmonic method (section (1.2.2.2)).

Figure 3.2 .7 shows the field produced by models for which the product of magnetization and topography is constant. The models were generated by multiplying each topography value of model $I 2$ by the factor $2 / K$ where $K$ varied from 1 to 5 . Hahn's, approximate relation holds quite well until the relief becomes larger and the higher order terms in (2.6.2.4) cannat be ignored. Figure 3.2 .8 shows the further ambiguity of 'magnetization and topography by inverting the same - data specifying different magnetizations; the magnetization can be increased'without limit and a solution will be obtained at the expense of attenuated relief. As 
1

124 
-
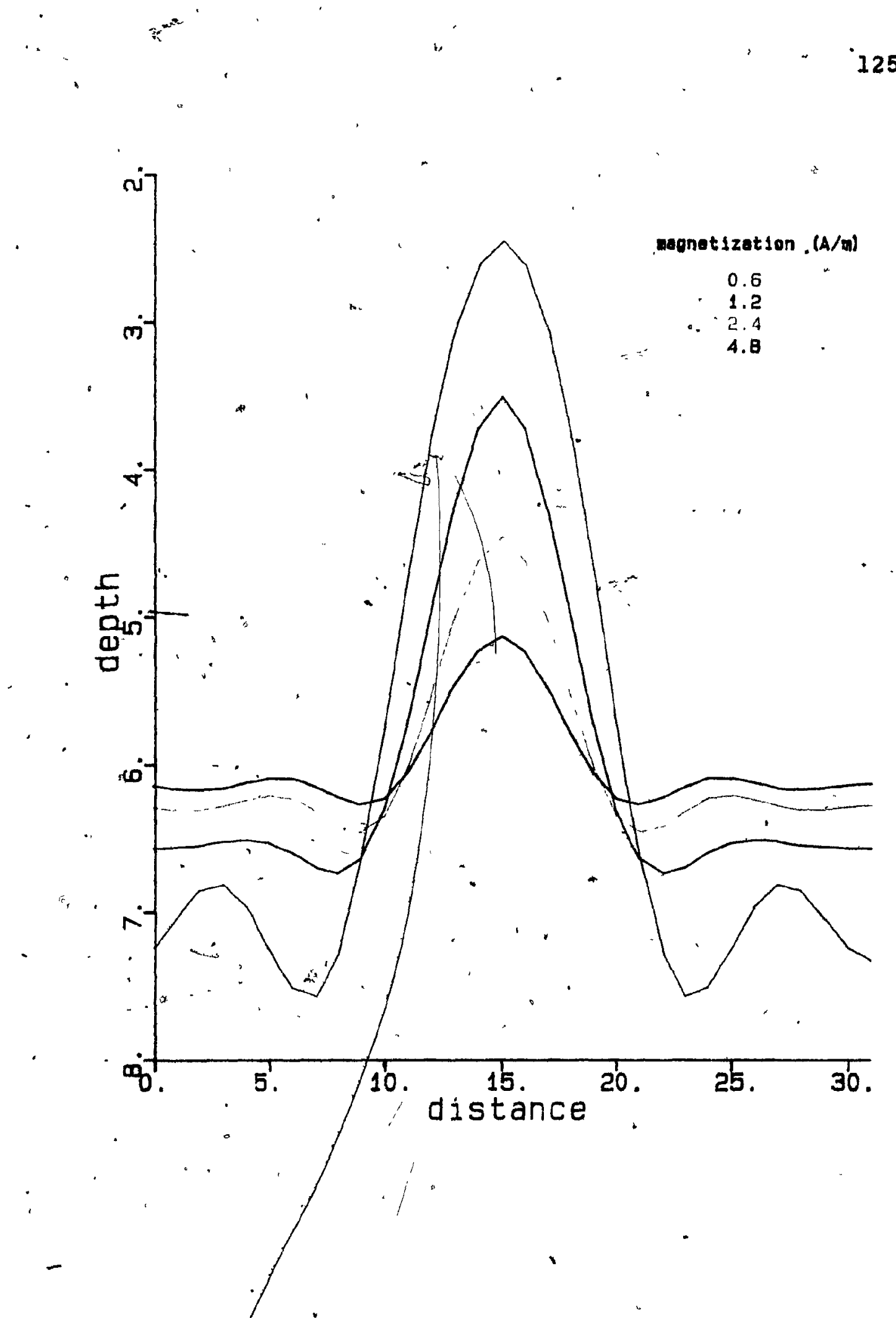

Figure 3.2.8 Results of inverting model L2 for various magnetization values. 
$J \rightarrow \infty$, an equivalent source distribution is obtained, provided the specified depth is above the shallowest source causing the observed field; only the first term in (2.6.2.4) is relevant and the relation between field and topography or magnetization approaches linearity (e.g. Roy, 1966). A lower limit on the maquetization exists, theoretically due to the restriction of the interface to the region below the observation plane and practically because of unacceptable RMS values.

Fortunately, if the layer depth $z_{0}$ is well constrained, a trial and error method can be used for the determination of magnetization $J$. For maximum efficiency only the first iteration of the algorithm (FLAT) is used. With ' $z_{0}$ approximately known, $J$ is varied over its possible range and the RMS error (equation (2.6.4.1)) found for each" approximate solution. The FLAT is sufficient for outlining - possible values of J. Figure 3.2 .9 shows the histories of this approach for model $L 2$ where a depth of 6 units is the correct value. Changing the damping factor does not usually obscure the lower limit on magnetization which as shown is quite sharp. The slow increase of the objective function as $J$ is increased is indicative that iterating using the algorithm (2.6.3.7) and/or relaxing other auxiliaryi parameters would result in an acceptable solution.

The fundamental ambiguity of the data is further exemplified by the lack of uniqueness in the resulting * 


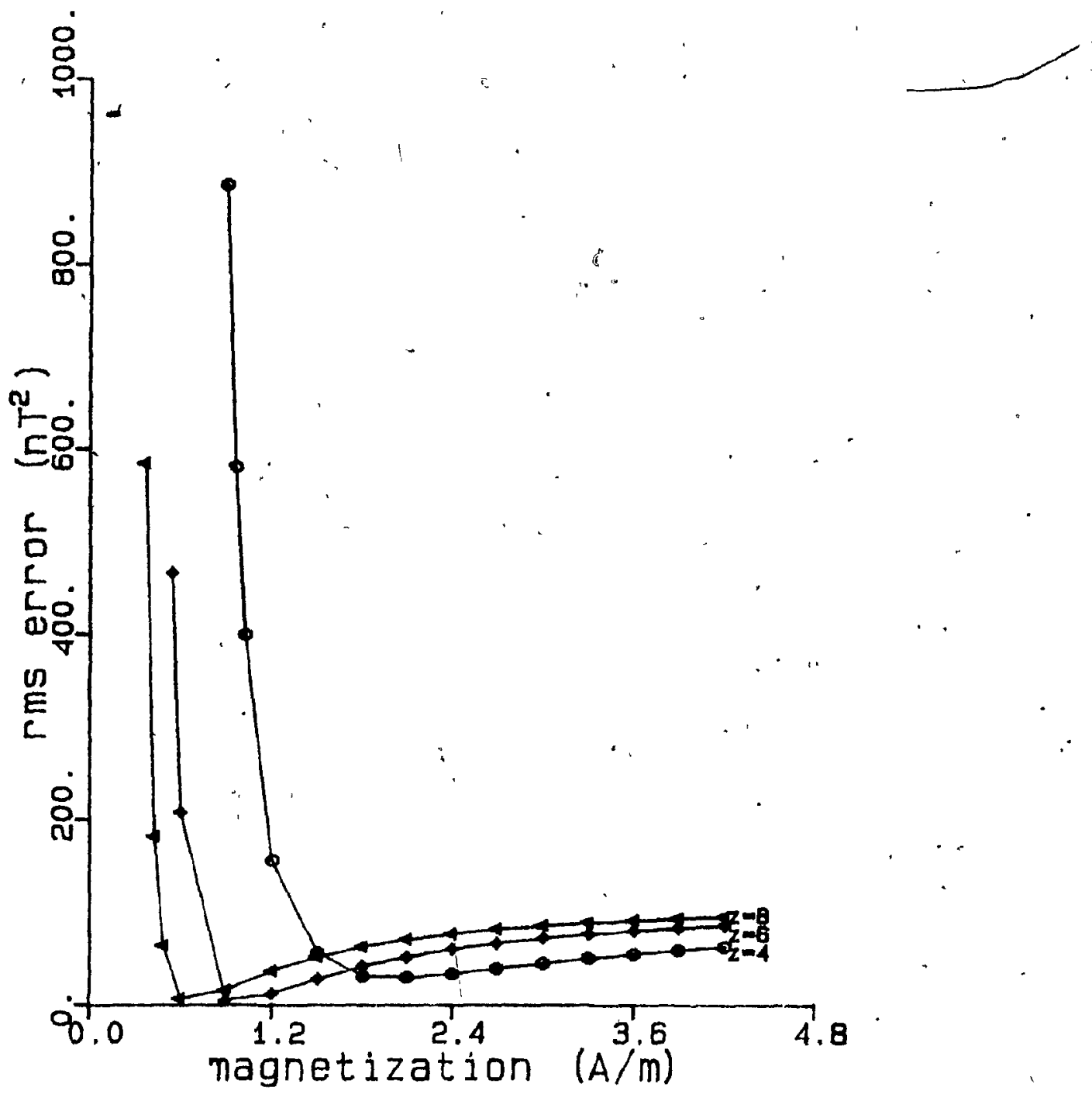

Figure 3.2.9 Dependence of RMS error on magnetization for the FLAT of model L2. The three curves correspond to different estimated depths given by the value of $z$ in the figure. 
solutions when $J$ is known. Figure 3.2 .10 shows a suite of acceptable solutions (all satisfying the data to within an RMS of $1 \mathrm{nT}^{2}$ ) obtained for model $\mathrm{L} 2$ data as the layer depth is varied. As expected, the topography becomes more oscillatory with depth. The correct depth in this example is 6 units. This coincides with the onset of the oscillations and would suggest that this property could be used as a prognosticator of the true depth. A similar effect is observed in downward continuation (Roy, 1966). However with real, noisy data it cannot be expected that small effects such as this could be distinguished from noise amplification plus the fact that the true topography is not necessarily smooth. As $z_{0}$ is increased the relative depth to the top of the relief decreases until a limiting depth is reached (Skeels, 1947). Inversions for depths greater than this value do not produce acceptable solutions. The minimum depth allowed is reached when the relief approaches the observation level.

With a lower limit on magnetization and an upper lifit on depth it is apparent that a hyperbolic-like boundary separates the region of acceptable and unacceptable solutions in RMS space (Courtillot et al, 1974; Quereshi and Mula, 1976). Table 3.2 delineates this boundary for the example data of model $L 2$. The correct values are $z_{0}=6$ and $J=1.2$. 


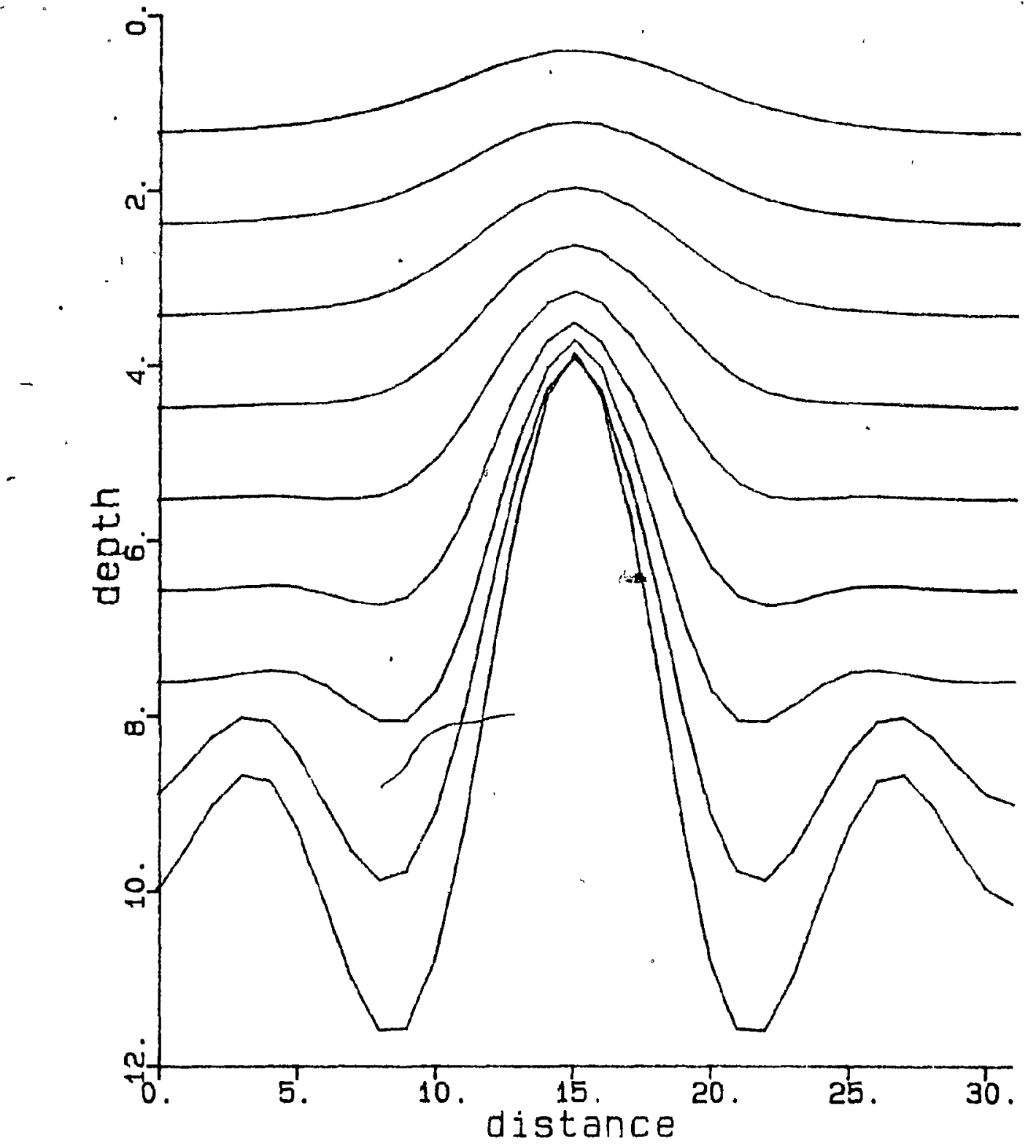

Eigure 3.2.10 Results of inverting model L2 for various layer depths. Average layer depth ranges from 1 to 9 units. 
Table 3.2

Summary of inversions for L2. Values of final RMS error are shown as a function of $J$ and $z_{0}$. Divergent solutions are denoted by div.

\begin{tabular}{|c|c|c|c|c|c|}
\hline & 0.3 & 0.6 & 1.2 & 2.4 & 4.8 \\
\hline 2 & div & $<1$ & $<1$ & $<1$ & $<1$ \\
\hline 4 & div & $<1$ & $<1$ & $<1$ & $<1$ \\
\hline 6 & div & $<1$ & $<1$ & $<1$ & $<1$ \\
\hline 8 & div & div & 83 & $<1$ & $<1$ \\
\hline 10 & div & $\operatorname{aiv}$ & div & 2 & 6 \\
\hline
\end{tabular}

Unfortunately Table 3.2 suffers from the limitation that the depth and magnetization values have been specifically chosen; only a predetermined area of parameter space is investigated. For a more comprehensive examination, a Monte-Carlo search has been used. Depth (d) and susceptibility (s) values for inversion were generated according to

$$
\begin{aligned}
& d=d_{0} 2^{r} \\
& s=s_{0} 10^{r}
\end{aligned}
$$

where subscript 0 denotes the starting values and $r$ is a random number. $r$ was provided by the MCGill subroutine RNOR which generates a normally distributed sequence of numbers with zero mean and unit variance. Consequently, '68\% of the 
generated $s$ values, 1 ie between $s_{0} / 10$ and $10 s_{0}, 688$ of the d's lie between $d_{0} / 2$ and $2 d_{0}$. Magnetizations $(m)$ were calculated from $m=s T$, where $T$ (the total field) was taken to be $60000 \mathrm{nT}$, so that a susceptibility of 0.002 emu corresponds to an induced magnetization of $1.2 \mathrm{~A} / \mathrm{m}$. Following Jones and Hutton (1979), when an acceptable model (at the InT2 RMS level) is found from inversion, $s_{0}$ and $d_{0}$ are replaced with the most recent values. This decreases the dependence on the starting model values. For physical reasons a maximum suseptibility of 0.01 emu was aflowed in the search. Figure 3.2 .11 shows the depth and susceptibility values that resulted in successful inversions of model L2 data. The search was initialised with $d=3$ and $\mathbf{s}=0.001$. From 1500 tested models, 295 have produced RMS errors less than $\ln \mathrm{T}^{2}$. Curve $B$ defines the limit delineated roughly in Table 3.2 while curve $A$ is a limit imposed by the finite depth of the layer: the magnetization must be large enough so the corresponding relief lies below the observation plane.

The trial and error approach for determining magnetization outlined above is also useful for deducing the maximum depth for inversion once a magnetization value is specified. Figure 3.2 .12 shows the RMS error of the FLAT as a function of the average layer depth for differing magnetizations. Again, the boundary between non-acceptable and allowable solutions is sharp and occurs at a greater 


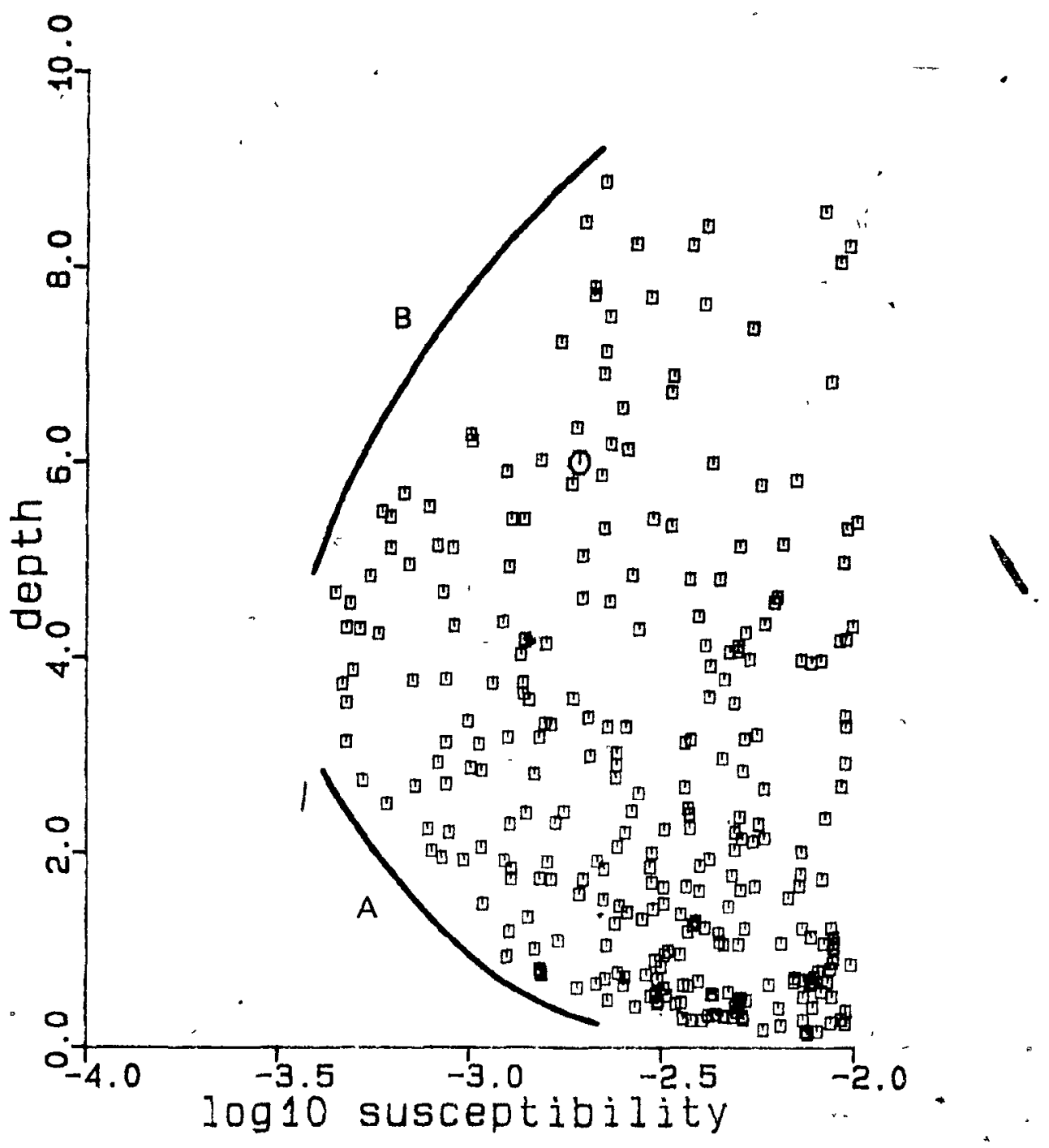

Figure 3.2.11 Results of Monte-Carlo search in depth-susceptibility space for model L2. Each acceptable model is shown as a square. The circle denotes the true values from which $L 2$ data was generated. 


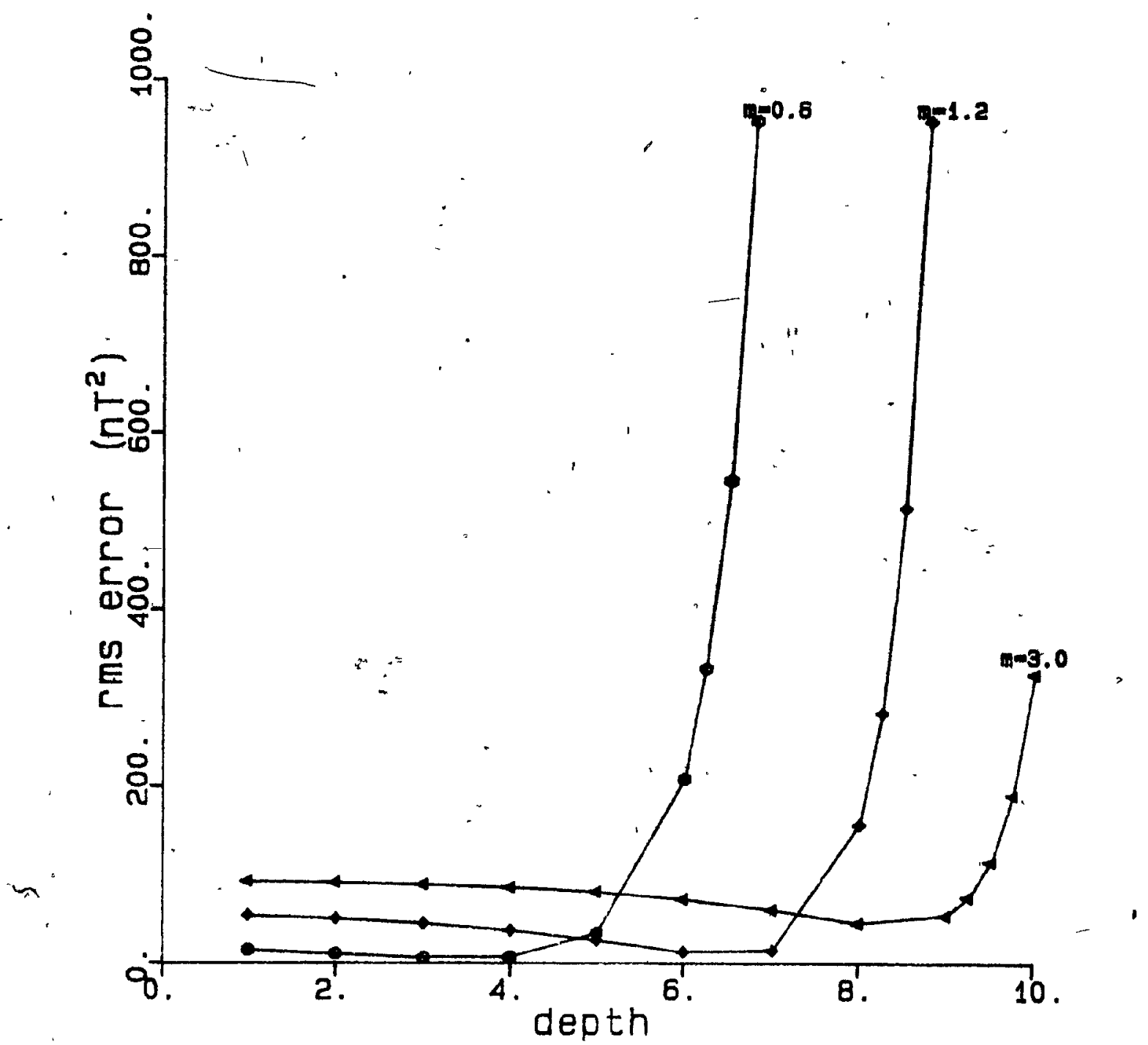

Eigure 3.2.12 Dependence of RMS error on depth for the FLAT of model L2. The three curves correspond to different estimated magnetizations given ${ }^{\prime}$ by the value of $m$ in the figuré. 
depth as magnetization increases. For $J=1.2$ (the correct value), the boundary coincides with the limiting depth from Figure 3.2.10.

\section{3 Relaxation of initial assumptions}

\subsubsection{Variable magnetization.}

From (2.6.2.4) it is apparent that amplitude modulation of the calculated field is imposed by the magnetization distribution $J(r)$. The most serious effects of assuming incorrectly a constantly magnetized source would therefore be expected when the topography $h(r)$ and its magnetization are correlated. This is not an unexpected situation, for example, a dyke exhibiting a large magnetization occuring within and above the basement. However this type of feature should be recognized, due to its greater anomaly amplitude, prior to inversion, modelled and removed.

In the expansion of $(2.6 .2 .4)$, the magnetization function is present only to first order; the topography is the dominant term so small variations of $J(r)$ about its mean value should not cause serious problems. Figure 3.3.1a shows the results of inversions of three data sets generated from the topography values of model LI (Figure 3.2.3) and having the following magnetization functions:

$$
\begin{aligned}
& \text { AM1 }=1.2+0.2 \cos (.125 \pi(i-1)) \\
& \text { AM2 }=1.2+0.2 \cos (.375 \pi(i-1))
\end{aligned}
$$



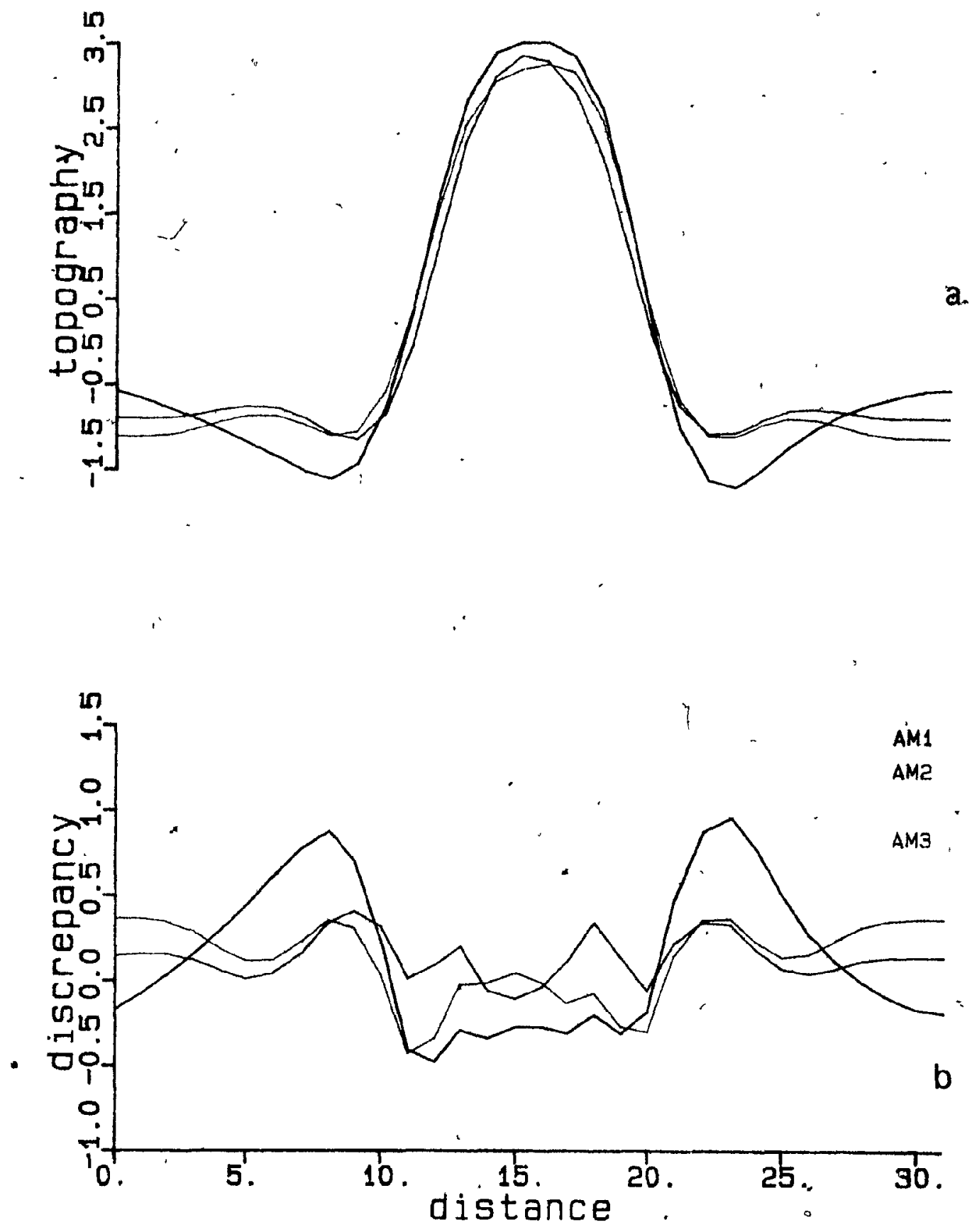

Figure 3.3.1 Effects of variable magnetization. Solutions for the magnetization distributions AMI, AM2 and AM3 (see text) are given in a. Difference between estimates in a. and the true solution are given in $b$. 


$$
A M 3=1.2+0.2 \cos (.5725 \pi(i-I))
$$

with $n$ the number of data (32 for this example). Figure $3.3 .1 \mathrm{~b}$ shows in detail the discrepancies between the estimates in Fig. 3.3.1a and the true model. As expected, the most serious departures occur with AMI, the longer wavelength magnetization that correlates most with the topography. The short wavelength magnetization of AM3 produces the smallest error, with the discrepancies being confined to the frequencies within the passband of filtering imposed by damping the solution. The damping was constant for all three inversions above. In all three cases, the maximum deviation from constant magnetization was 168 of the constant value. The results show the relative insensitivity of inversion to minor relaxations of the assumptions on the model magnetization.

\section{3 .2 Noise}

All aeromagnetic measurements suffer degradation in quality due to the presence of non-geologic noise, that is, that part of the measurement not attributable to geologic features of interest. Unfortunately, the sensitivity level of available instruments only sets a lower limit to the possible error in actual readings so this cannot be used as a practical noise estimate. The possible sources of noise range from surveying effects such as instrument noise, aircraft noise (magnetic and electrical), motion of the 
aircraft and sensor, location érrors and aliasing, to processing effects, such as levelling; gridding, diurnal effects and removal of the core field.

Usually, the simplifying assumption, is made that these effects are random in nature, and as such can be classed as white noise. In order to investigate the consequences of noisy data on inversion, samples of white noise with a variance of $100 \mathrm{nT}^{2}$ were added to the response of model L2 (Figure 3.2.3). This corresponds to approximately 108 noise. Figures $3.3 .2 b$ and $3.3 .3 b$ show the two noise samples, while Figures $3.3 .2 a$ and $3.3 .3 a$-illustrate the results of inversions on the respective noisy data sets. In comparison with the noise-free inversion, also plotted, the algorithm has attempted to model the noise but only succeeds in - producing a fit to the long wavelength noise components, specifically that passband defined by the level of damping incorporated into the solution construction. This is demonstrated by the final RMS error (the value of which is shown' in the figures) being less than the noise variance. Similarly, in Figure 3.3 .3 , in the range 0 to 7 distance units, the noise approximates a sinusoidal form with a peak at 0 unit and a trough at 4 units. The noisy solution reflects this form with shallower estimafes occuring at the noise peak and deeper estimates at the noise trough:

Inaccurate removal of the global geomagnetic field tends to produce long wavelength errors. Models of the geomagnetic 

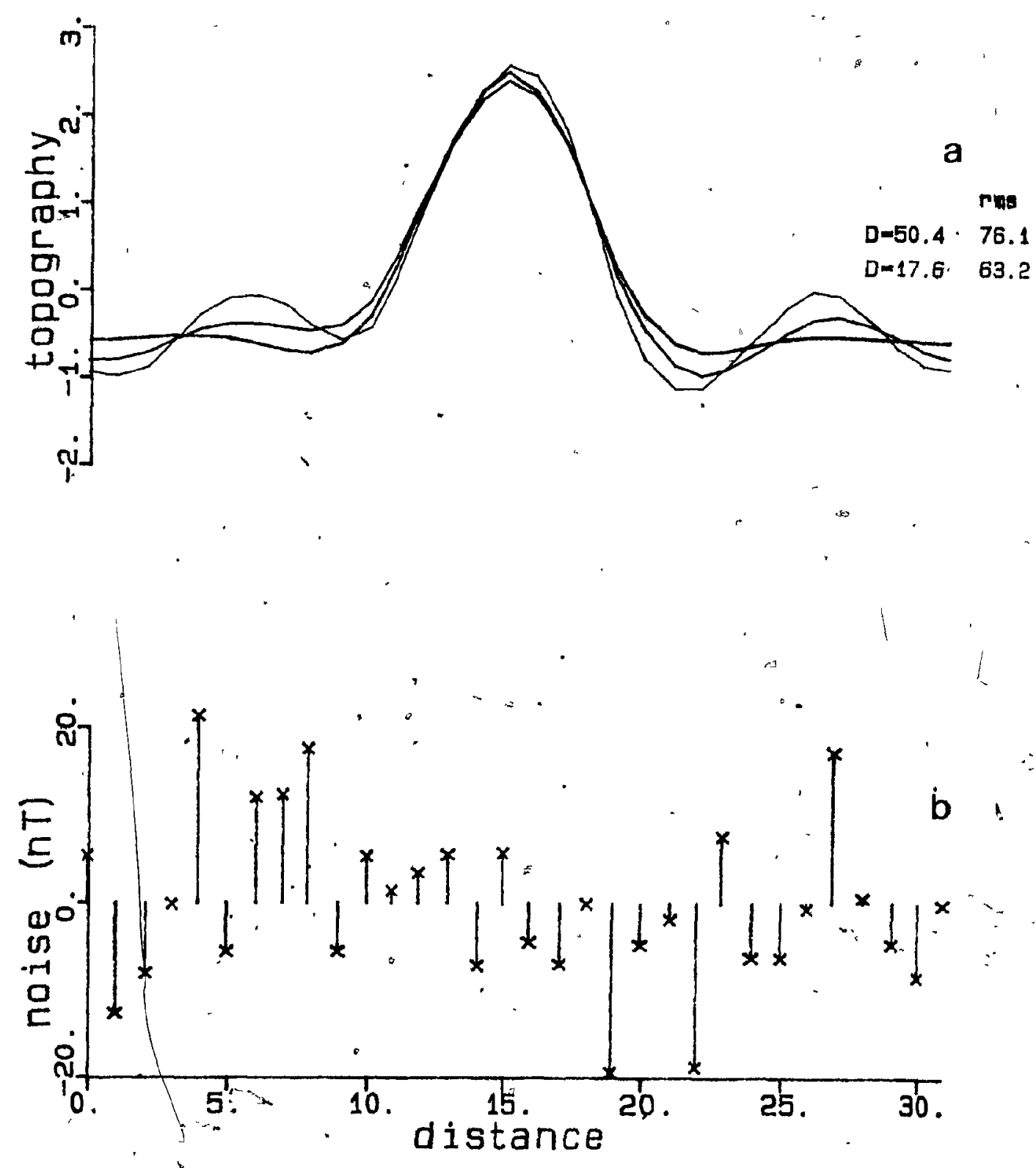

Pigure 3.3.2 Effects of noisy data. Black line in (a) denotes noise-free inversion. Coloured lines are inversions for damping factors given by $D$. White noise sample added to data is shown in (b). The final RMS error for inversions is given by the value of rms. 

field are usually represented as a spherical harmonic series. derived from a least squares fit to a large number of observations of the main field. Such models are only approximations to the true field at a specific time (e.g. Barraclough, et al, 1975) and contain errors of fit. Additionally, errors accrue when these models are extrapolated in time due to deficiencies in the secular variation component. As 'solution damping is a low-pass filtering operation, these long wavelength errors are expected to cause more serious effects in the topography estimates. Figure 3.3.4a shows the solution for the low frequency noise sample in Fig. 3.3.4b. The sample was generated from white noise which was then filtered with a low-pass filter whose cut-off frequency was within the 1 imits imposed by damping. Hence, all noise is modelled and the final RMs error is acceptable at the $\ln \mathrm{T}^{2}$ level.

Random errors can be combatted effectively by inspection of the energy spectrum of the data; the noise variance can be estimated to be the level of the flat part of the field spectrum at high frequencies. Wiener filtering can then be used to remove such components from the observations (e.g. Gupta and Ramani, 1980). Long wavelength noise poses a much more difficult problem whose effects can only be mitigated if the features of interest are not of a comparable wavelength. 

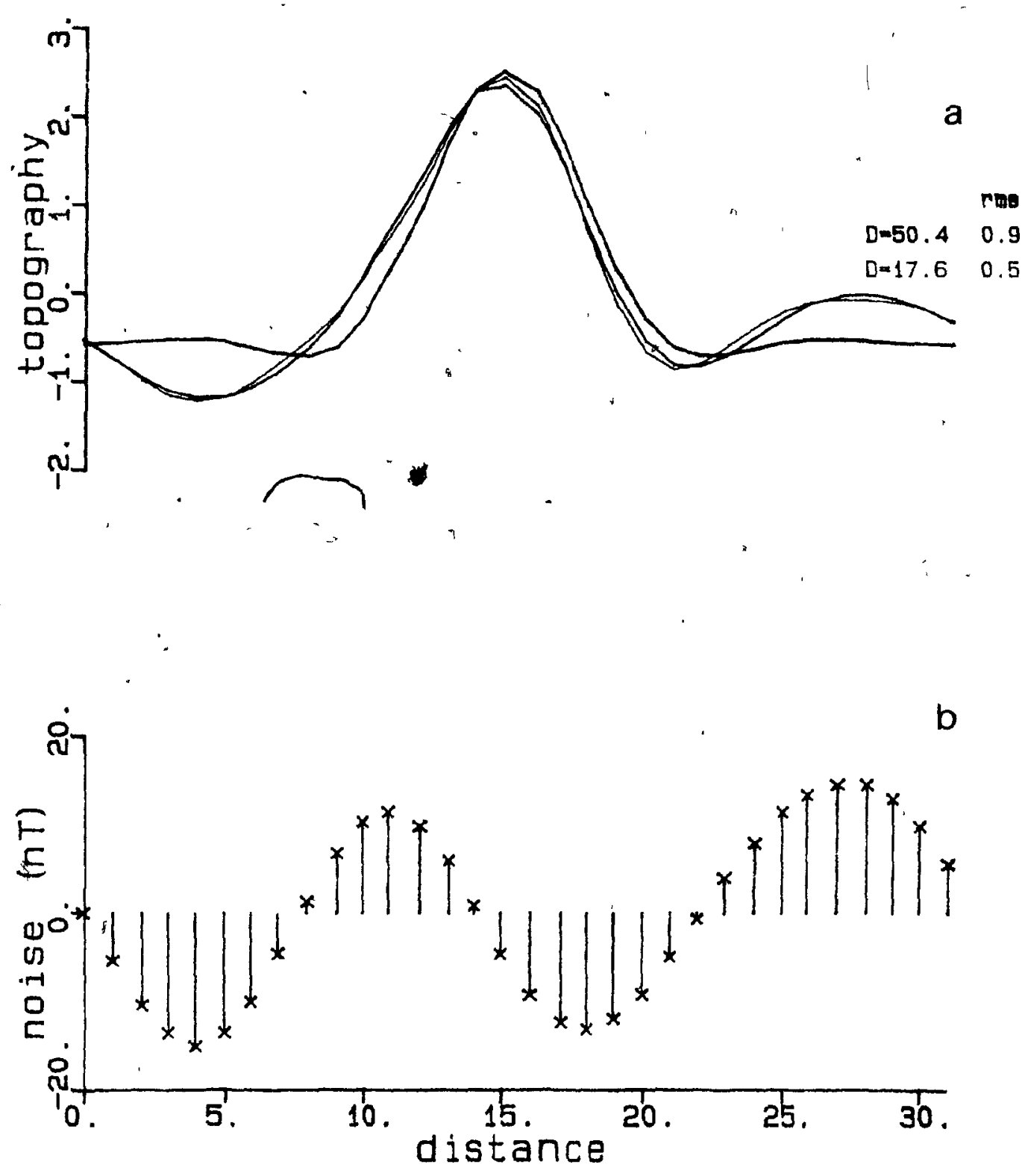

Pigure 3.3.4 Effects of long wavelength noise on inversion. Black line in (a) denotes noise-free inversion. Coloured lines are inversions for damping factors given by $D$. Noise sample is shown in (b). 


\subsubsection{Starting models}

A stringent test of the uniqueness of the final model estimates is to address the capability of the algorithm to produce solutions that are not dependent on the form of the starting model. All preceding inversions have been initialised with a flat starting model $(h(r)=0)$. At this point in parameter space the partial derivatives given by the decomposition $(2.6 .3 .7)$ are correct. "Previous examples have shown that this is a sufficient approximation to the true Jacobian at other points in parameter space to ensure convergence to an acceptable solution. Similarly, initialising the method with models other than $h(r)=0$ would not be expected to hinder convergence, so the question of uniqueness can be addressed.

Figure $3.3 .5 b$ shows a variety of smooth sinusoidal starting models with the corresponding solutions given in Figure 3.3.5a for model. L2 data. All RMS errors are less than $1 n T^{2}$ and match the true solution well. A minor departure is noted for model SM3 which is explained by the effects of Fourier transformation at the data set edges. Figures 3.3 .6 and 3.3 .7 give the results of inversions based on starting models exhibiting more serious departures from the usual flat one. Models SM4 and SM5 result in an acceptable approximation to the true relief except for discontinuity effects at data edges. SM6 and SM7 (Figure 3.3.7a) both fit the data to within $\ln \mathrm{T}^{2}$ but show a poor 


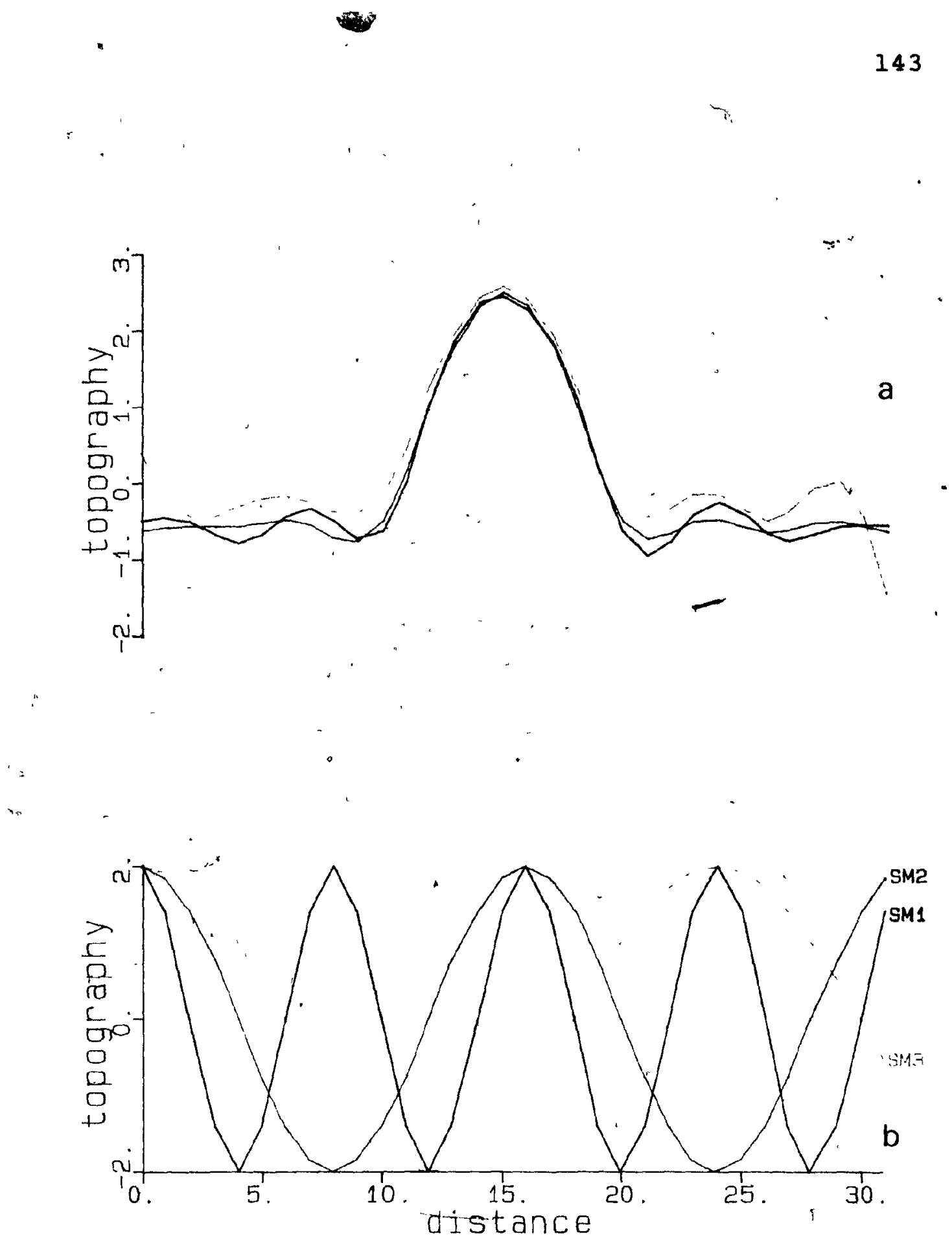

Figure 3.3.5 Effects of starting models. Inversions of model I2 are given in (a) with the corresponding starting model in (b).

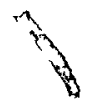


$\therefore$

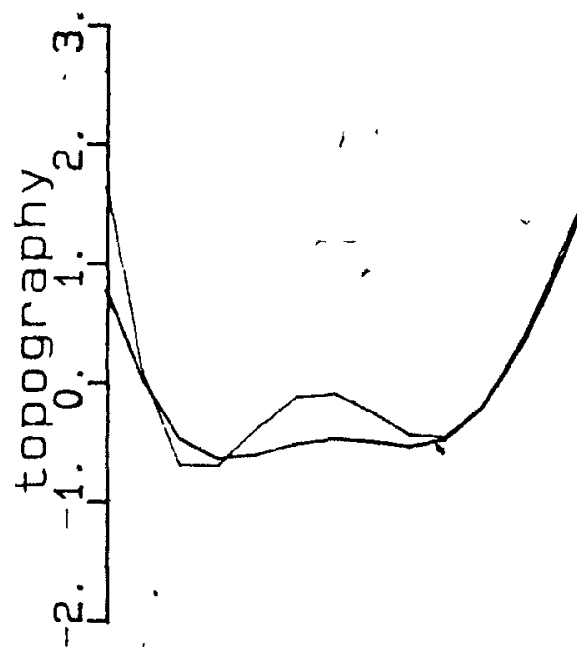

a

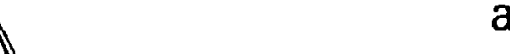

$\mathbb{1}$
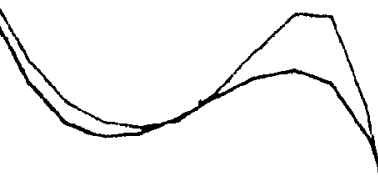

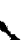

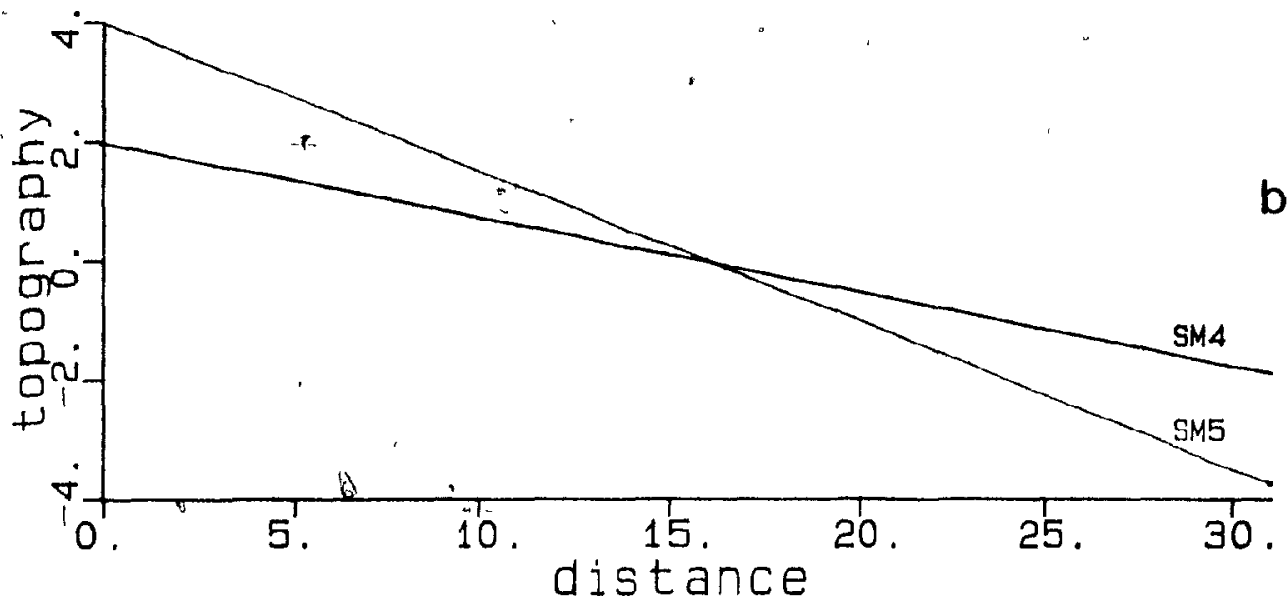

Figure 3.3.6 Effects of starting models. Inversions of model L2 are given in (a) with the corresponding starting model in (b). 

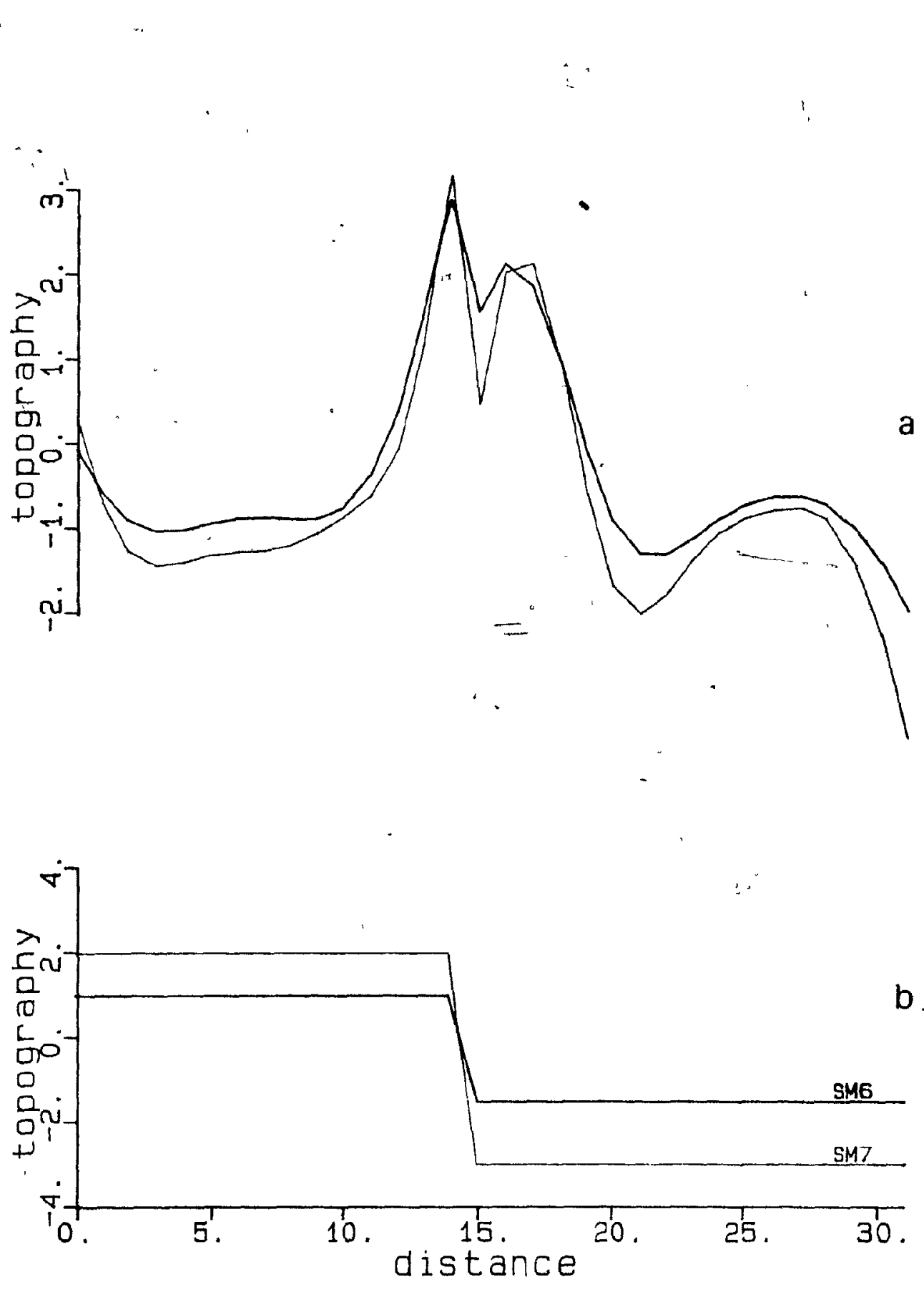

Eigure 3.3.7 Effects of starting models. Inversions of model L2 are given in (a) with the corresponding starting model in (b). 
match to the real topography. Both these starting models contain a discontinuity at the centre of the profile where the 'hump' of the true topography is located. In these cases this feature has not been entirely removed during inversion showing dependence on the initial model. The major characteristics of the true model appear, but high frequencies introduced by the discontinuity cause some degradation. Fortunately, the step model is an extreme case and would usually be avoided due to the algorithm's incapability of reconstructing such a.feature.

\section{$\underline{3} \cdot \underline{4}$ Comparison with a fully nonlinear approach}

Before considering the consequences of using a constant Jacobian throughout the iterative process, the question of how the calculated solution relates to the model is adaressed. "This is done with the stipulation that recovering the model from a set of calculated data does not always guarantee the success of the method when applied to actual data.

In section (3.2.1) the necessity for damping within the inversion algorithm to suppress oscillatory character (associated with small eigenvalues at high wavenumbers) in the solution was emphasised. The ratio of inverse to damped inverse eigenvalues in Figure 3.2.I specifies the low-pass filtering effect (shown in the wavenumber domain) when the 
inverse $A^{+}$is applied to the data (and residuals). Ail wavenumber components above the cut-off wavenumber are removed from the data, and hence, the solution: components of the true topography at higher wavenumbers cannot be recovered. The accuracy of the computed solution therefore depends on whether or not the equivalent passband of the low-pass filter (Figure 3.2.1) includes all true topography wavelegths. Figure 3.4 .1 shows the amplitude spectra of the model and the computed solutions (with varying degrees of damping) for model L2. Figure 3.4.1 shows that as damping decreases, the computed spectrum approaches the true one at low wavenumbers. The damping factor of 17.6 corresponds to the fourth eigenvalue and the resultant filtering due to such a value is given by the curve ID=4 in Figure 3.2.1; this shows, that the cut-off wavenumber occurs around the seventh eigenvalue, or a wavenumber of 1.0 radians. Figure 3.4.1 confirms the surpression of wavenumber components greater than 1.0 in the computed estimates. A surprisingly good fit is obtained between the true.and computed solutions (shown in Figure 3.4.2) even though the discrepancy between amplitude spectra is lárge at high wavenumbers.

No theory can predict whether or not a constant Jacobian is a good enough approximation to the true one at all points in parameter space to ensure convergence of the algorithm to an acceptable solution. The examples of section (3.3.3) have dembnstrated that it is acceptable for smooth models; this ) 


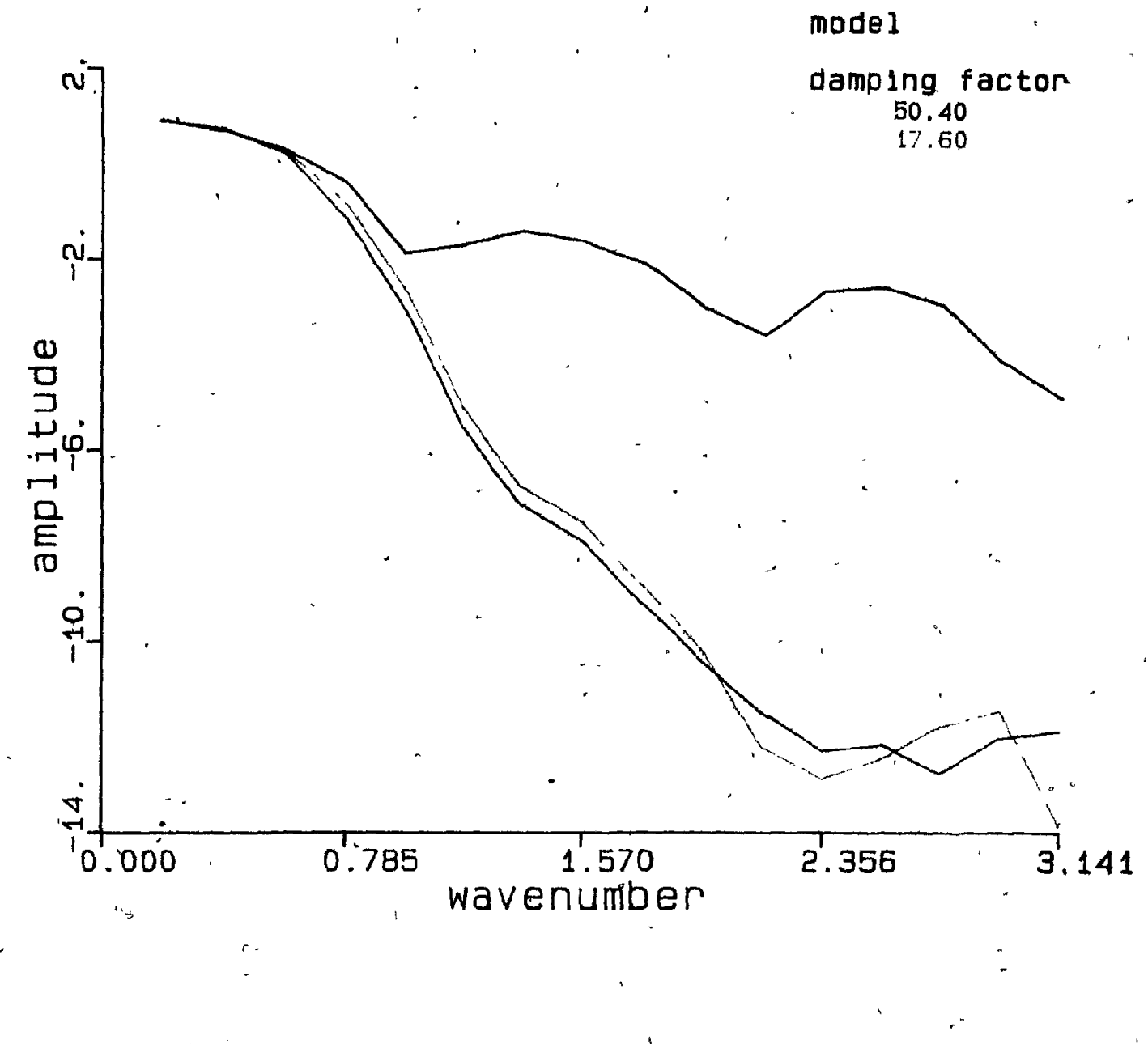

Iigure 3.4.1 Comparison of model and computed (for varying - ) solution amplitude spectra for model L2. 


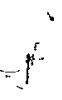

$\approx$

1

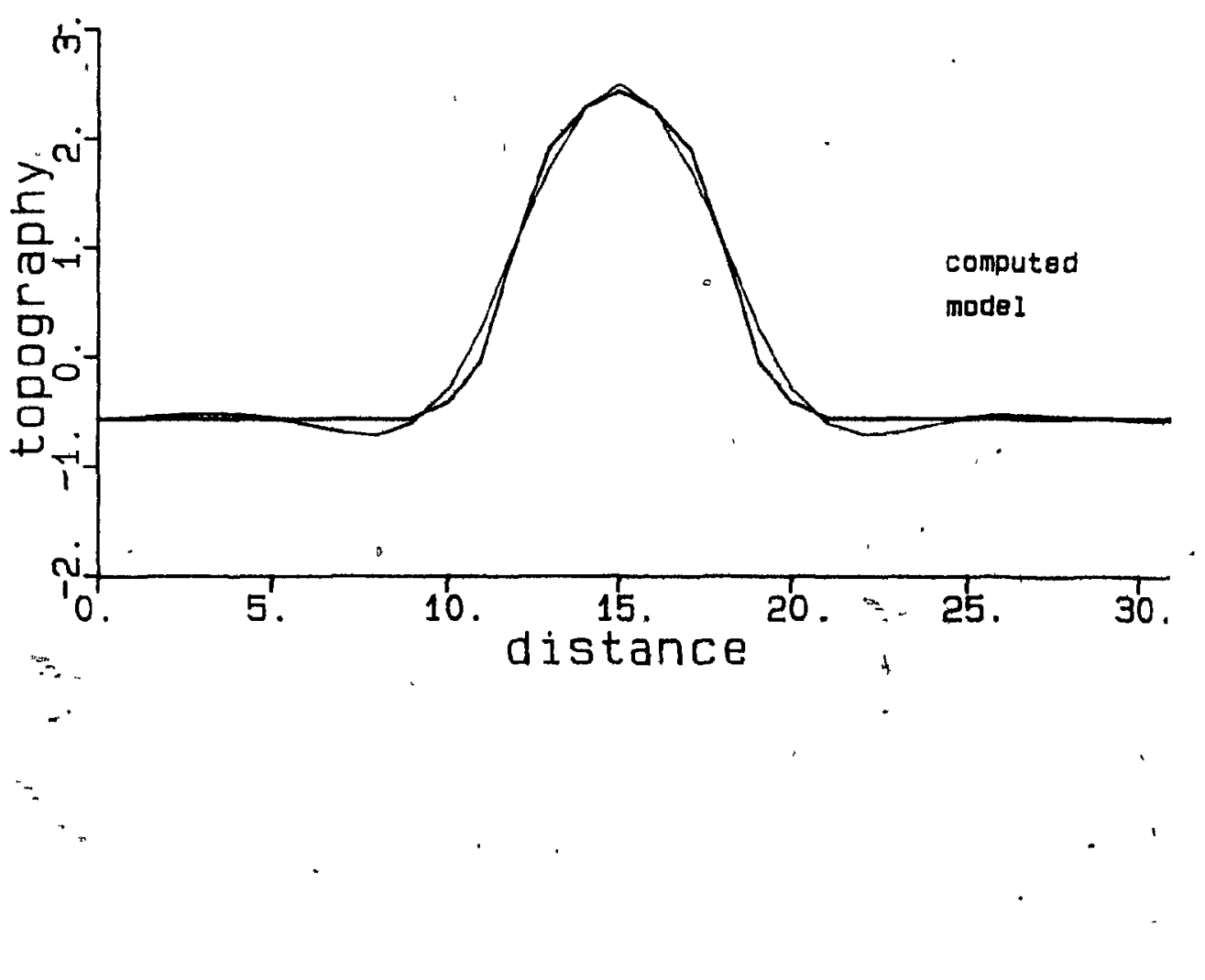

. Pigure 3.4 .2 Comparison of model and computed solutions $(\theta=17.6)$ for madel L2. 
would be expected because the method surpresses high wavenumber components in the data through damping. For model L2, in comparison with a standard nonlinear inversion programme which uses numerically determined svDs at each iteration (curve SVD in Figure 3.4.3), the proposed method (curve TOPO) compares favourably with the major aifferences" occuring at the deepest parts of the topography. The damping factor used for Figure 3.4 .3 was 50.34 (equal to the largest eigenvalue), other damping "factors produced very similak results. A comparison of the convergence of the two approaches is given in Figure 3.4.4. In test examples TOPO usually required more iterations than SVD. Table 3.3 compares the rates of convergence as a function of damping.

Table $3.3^{-}$

Number of iterations required to reach an acceptable solution (RMS error < $1 n T^{2}$ ) for. model L2 using programmes SVD and TOPO.

$\begin{array}{lrrrrr}\text { Damping factor } & 50.4 & 34.4 & 17.6 & 8.1 & 5.0 \\ \text { SVD } & 9 & 6 & 3 & 3 & 4 \\ \text { TOPO } & 16 & 7 & 2 & .5 & 11\end{array}$

When the computation time of the two methods is considered, the evaluation and subsequent inversion of the Jacobian in programme sVD results in the proposed method 


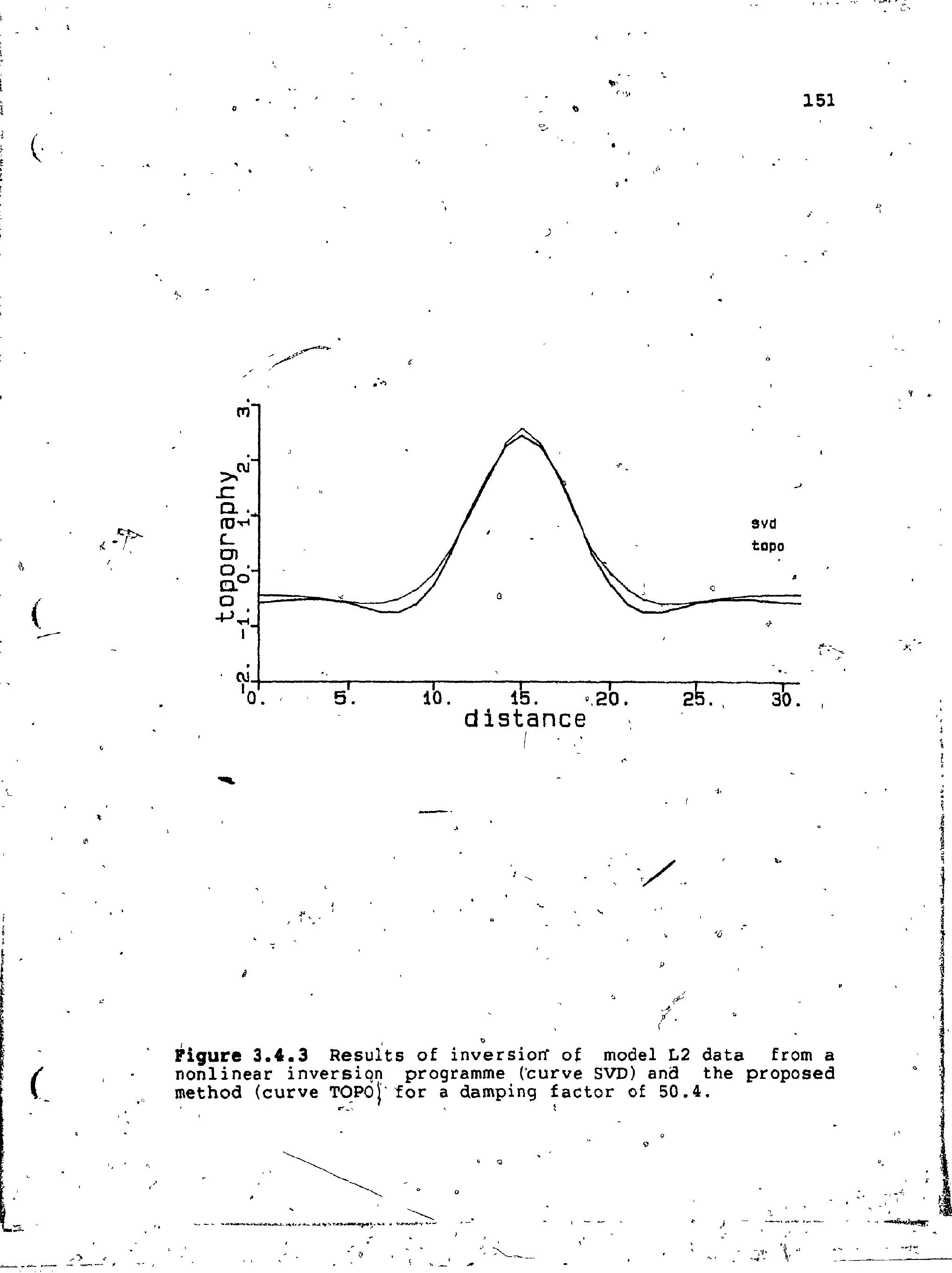


\&

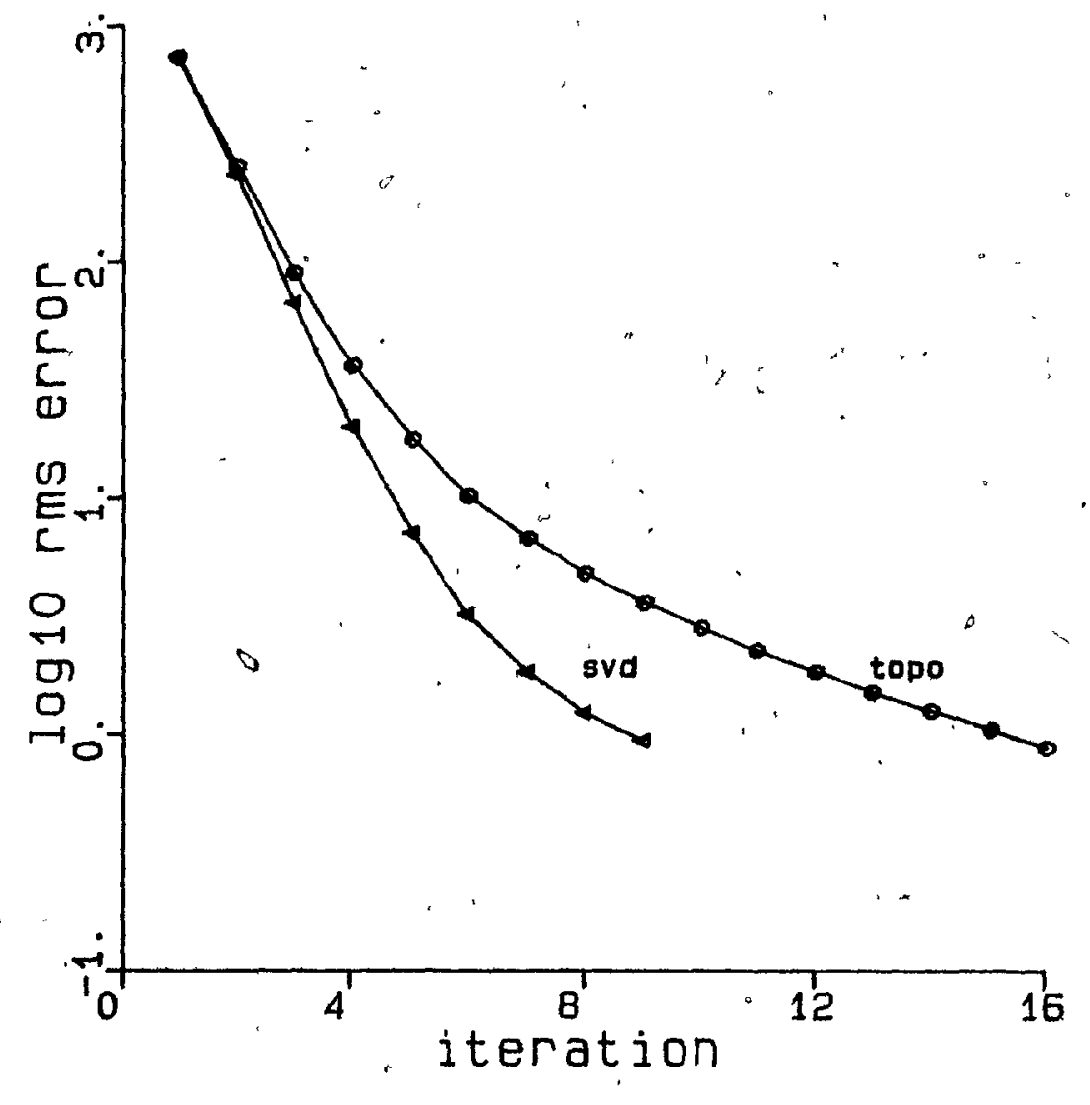

Figure 3.4.4 Comparison of iterative behaviour of RMS error for programmes SVD and TOPO.
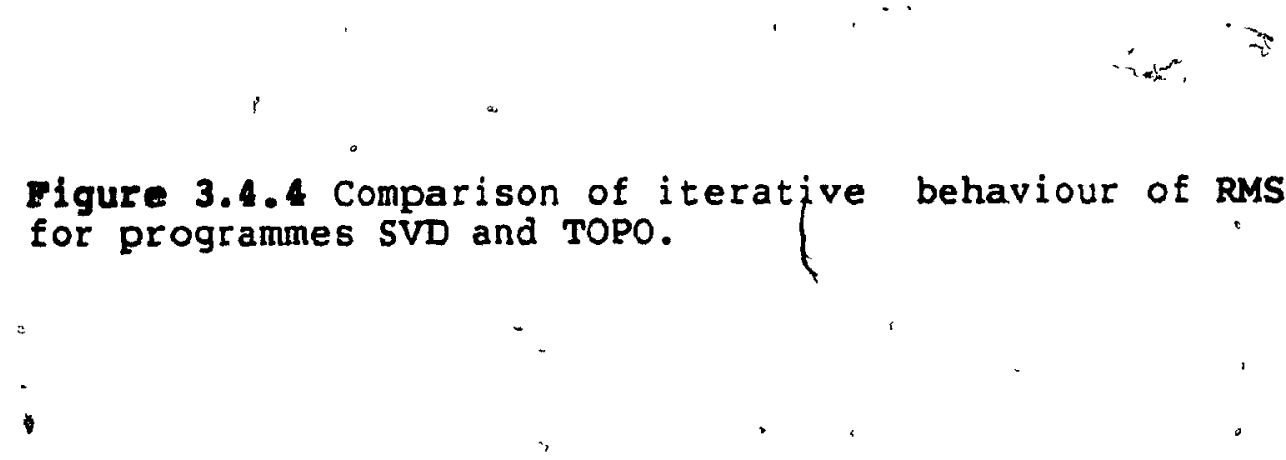
being an inexpensive but equally accurate alternative. As a rough guide to the difference in cost, a 32 point profile inverted with SVD required 45 seconds of CPU time (for 6 iterations). Programme TOPO solves approximately $140^{\circ}$ similar problems in the same time.

\section{$\underline{3} \cdot \underline{5}$ Practical resolution measures}

Due to the size of the matrices involved in processing map-size data sets, the numerical determination of the singular value decomposition of $A$ (evaluated at the final solution) or the determination of the eigenvectors of $A^{t} A$ for resolution calculations is not feasible. In section (2.6.6) the evaluation of the Jacobian at an acceptable solution was outlined with a view to determining the resolving power of small portions of data. If these areas are still too large to be practical, approximations can be introduced to reduce the computational load.

For the damped. case it was shown (section (2.6.3)) that the damped resolution matrix is determined from

$$
R=\frac{V \wedge^{2} v^{t}}{\Lambda^{2}+\theta}
$$

If $p$ is taken as the eigenvalue number where $\lambda^{2}<\theta$, then 


$$
\begin{aligned}
R & =\sum_{i=1}^{p} \lambda_{i}^{2 /\left(\lambda^{2}+\theta\right)} v_{i} v_{i}^{t}+\sum_{i=p+1}^{n} \lambda_{i}^{n} /\left(\lambda^{2}+\theta\right) v v^{t} \\
& =\sum_{i=1}^{p} \lambda_{i}^{2} /\left(\lambda^{2}+\theta\right) v_{i} v_{i}^{t}+\sum_{i=p+1}^{n} \sum_{i}^{2} / \dot{i} v_{i} v_{i}^{t}
\end{aligned}
$$

which implies that the majority of the resolving power is contained in the first p eigenvectors. Now when $\theta$ is large compared to the maximum eigenvalue of $A$, the damped inverse, $H$, tends towards the steepest descent inverse (section (2.4)), that is, $\mathrm{H} \rightarrow \mathrm{A} / \theta$, with $\mathrm{A}$ evaluated at an acceptable solution. Consequently,

$\begin{aligned} \quad R=H A & =\frac{A^{t} A}{\theta}=\frac{V \Lambda^{2} V^{t}}{\theta} \\ & =\sum_{i=1}^{n} \lambda_{i}^{2 / \theta} v_{i}^{*} i .\end{aligned}$

In comparison with (3.6), the discrepancy only occurs in the first $p$ eigenvalues. Writing $R$ (true) and $R$ (app) for the " true(3.5) and approximate (3.7) resolution matrices:

$$
R(a p p)=R(\text { true })+\sum_{i=1}^{p} \lambda+r\left[\left(\lambda_{i}^{2}+\theta\right) \theta\right] v_{i}^{t} v^{t}
$$

Using trace( $R$ ) as a measure of the number of independent parameters that are resolvable (Aki and Richards, 1980, p.698) 


$$
\operatorname{tr}[R(a p p)]=\operatorname{tr}[R(\operatorname{true})]+\sum_{i=1}^{p} \lambda_{i}^{4 / C} \underset{i}{\left.\left(\lambda^{2}+\theta\right) \theta\right]}
$$

showing that the approximate resolution measure"gives an overestimate of the true value. This discrepancy increases as $\theta$ decreases and $H$ tends away from the steepest descent inverse. Consequently, the number of independent parameter combinations resolved by the data set is best estimated from the original damped eigenvalue spectrum (2.6.3.8). For $R$ (true), its trace ís always less than $n$, the total number of parameters and the diagonal elements can be used as, a measure of the resolvability of each parameter (e.g. Aki et al, 1977). This approach is not feasible for $R(a p p)$.

$R$ (app) is, however, useful in finding bounds on the true - resolving widths, where width can be taken as the width of the resolving kernel at one half of its maximum value. An informative measure that responds to width size is given by the least squares error vector:

$$
\operatorname{res}_{k}=\sum_{i=1}^{n}\left(r_{k i}-\delta_{k i}\right)^{2}
$$

where $r_{k i}$ is an element of the resolution matrix $R$ and $\delta$ is the Kronecker delta. Converting resk into a relative error measure res $\dot{k}=1$ res $_{k}($ Crossley and Reid, 1982) produces a quantity varyling from 0 (unresolved) to $I$ (perfectly resolved parameter). res'k can be easily determined by orthonormalizing $R$ giving $R^{\prime}$. It then follows that 


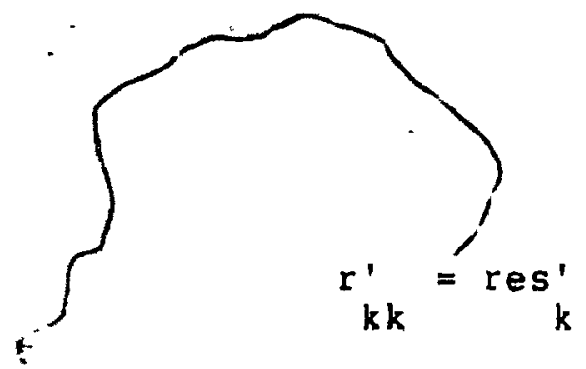

that is, the diagonal elements of $R^{\prime}$ give the discrepancy between each row and a delta function. Following Nolet (1978), res $\dot{k}_{k}$ can be converted, into another measure, $I_{k}$, that has the dimension of length and is given by $L_{k}=d x / r e s j_{k}$ where $d x$ is the data spacing. $L_{k}$ gives the averaging width of a box-car shaped resolving kernel centred at parameter $k$, assuming that the rows of $R$ are unimodular.

The unimodularity constraint is important for preserving resolving kernel scale when the rows of $R$ are not simply space-shifted versions of each other (as in the case of circulant A). Additionally, it ensures the computed averages are true ones. For circulant $A$, unimodular rows of $\mathrm{R}$ would be useful for comparitive purposes when different damping factors and/or auxiliary parameters are specified. However, for problem $T I$, the source model produces a dipolar field so the Frechet derivatives have zero area and the resolving kernels, being a linear combination of the derivatives, also have zero area. Thus it is not possible to enforce the unimodularity constraint. As a consequence of zero area resolving kernels, any bias in the solution is equal to the DC level of the data, which in our case is zero since the zero wavenumber component (corresponding to a zero eigenvalue) has been removed from the solution.

If $r^{t}$ denotes a row of the corresporiding resolution 
matrix, then

and

$$
\left\|r_{k}^{t}(a p p)\right\|=\left\|H(a p p) a_{k}\right\|=\mid\|\underset{\theta}{\wedge}\|^{t}\left\|_{k}\right\|
$$

$$
\| r_{k}^{t}(t \text { rue })\|=\| H(t r u e) a_{k}\|=\| \underset{-a \wedge^{2}+\theta}{\wedge} U_{k}^{t} a_{k} \|
$$

with $a_{k}$ denoting the $k$ th column of the Jacobian. Since all eigenvalues of $H(a p p)$ are greater than those corresponding in $H$ (true) and the norm of both $V$ and $U^{t}$ is one, it follows that

$$
|| r_{k}^{t}(a p p)\|>\| r_{k}^{t}(t r u e) \| \mid
$$

As the measure resk is simply the length of each, row, then

so

$$
\underset{k}{\operatorname{res}}(\operatorname{app})>\underset{k}{\operatorname{res}}(\text { true })
$$

$$
\underset{k}{\operatorname{res}^{\prime}}(a p p)<\underset{k}{\operatorname{res}}(\text { true })
$$

The orthonormalized resolution vector computed for $R_{\text {d }}$ (app) provides an upper bound to the resolving width for each parameter. More stringent bounds are obtained as the damping factor increases. Table 3.4 shows values of res $k$ calculated for the true and approximate resolution matrices corresponding to an acceptable solution of the model L2 (the solution is symmetric around parameter 16 , hence only half the values are shown). 
Table 3.4

True and approximate resolution measures for the solution of model L2.

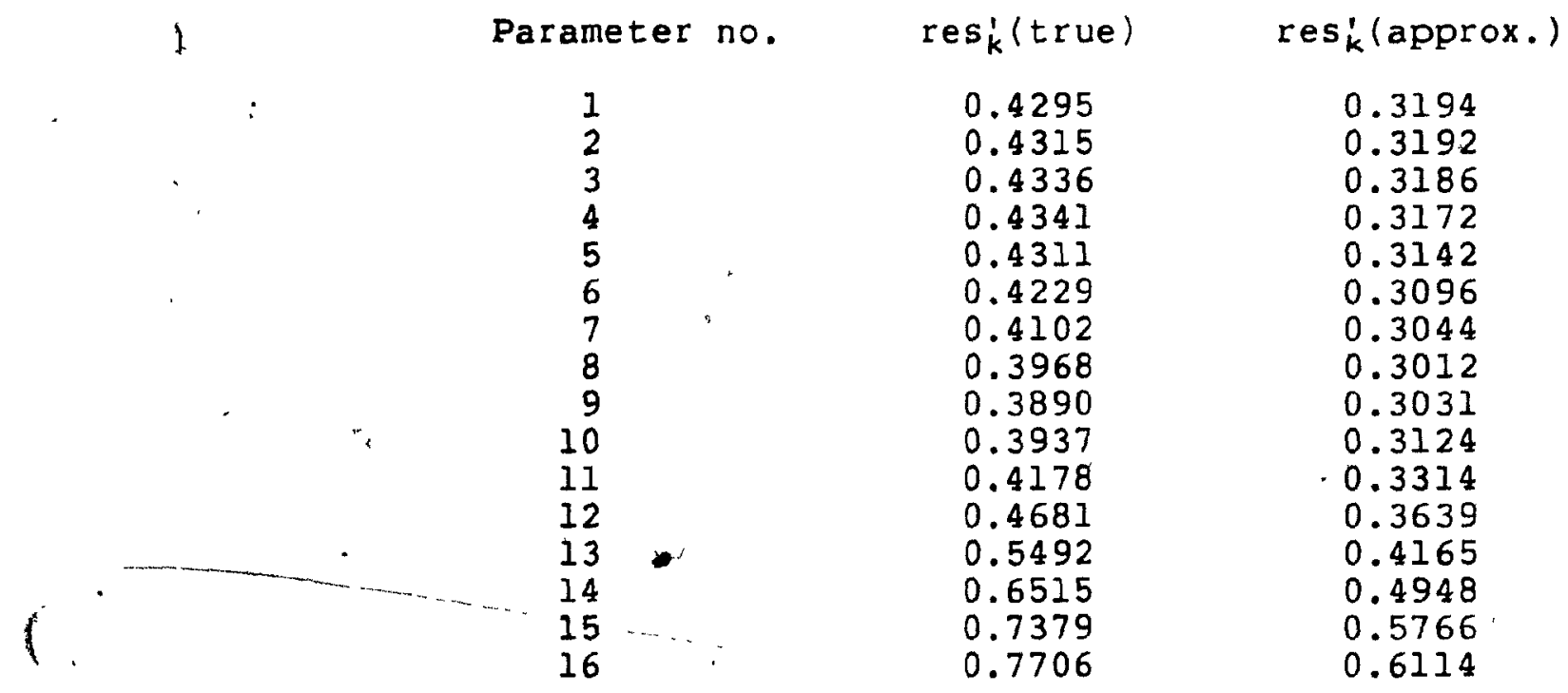

Similarly, Figure 3.5 .1 shows the rows of the two resolution matrices for parameters 10 and 16 (topography estimates at distances of 9 and 15 arbitrary units). The two sets of kernels show similar character but $R(a p p)$ provides consistently larger resolving width estimates. This is to be expected because $R(a p p)$ is simply the covariance matrix of the problem Frechet derivatives scaled by the damping factor. Hence it represents the worst resolution possible unless a disasterous choice is made for the inverse $H$.

Another, quicker, approach to obtaining approximate resolution measyres, is to compute the matrix (actually only one row is needed as in section (2.6.3)) 


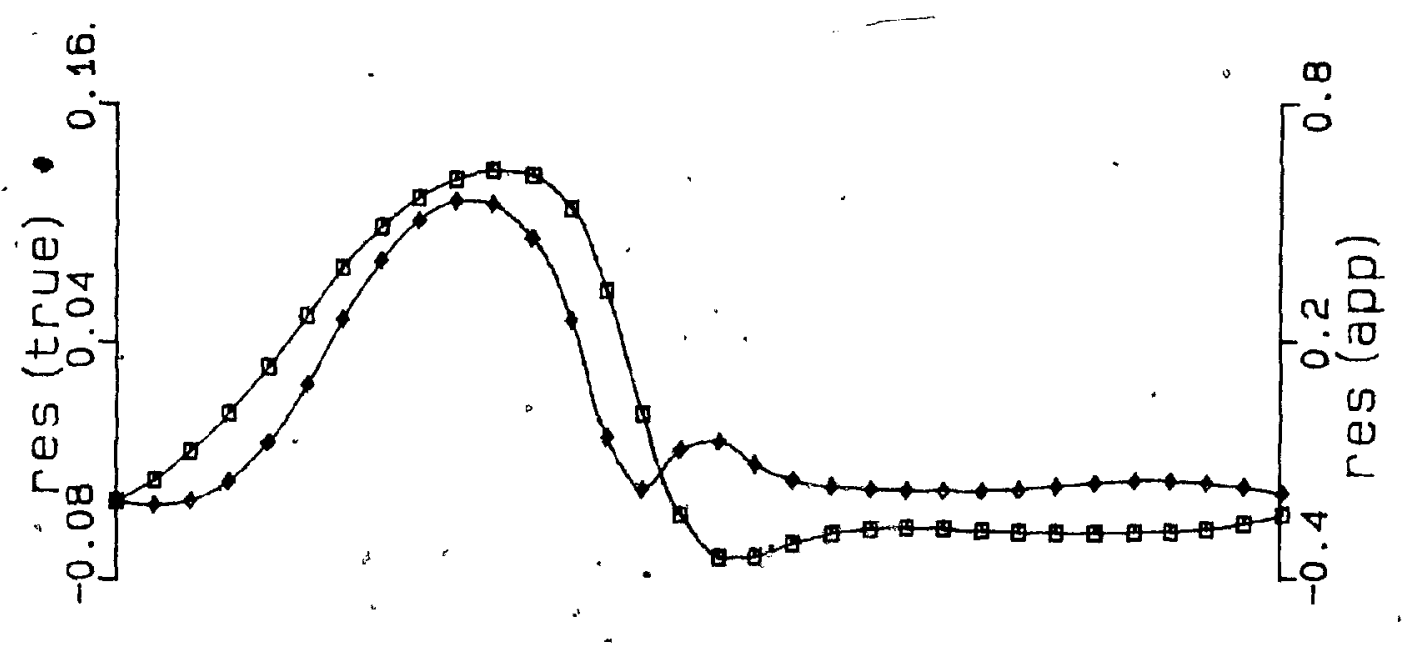

¿ approximate $\cdot$ true

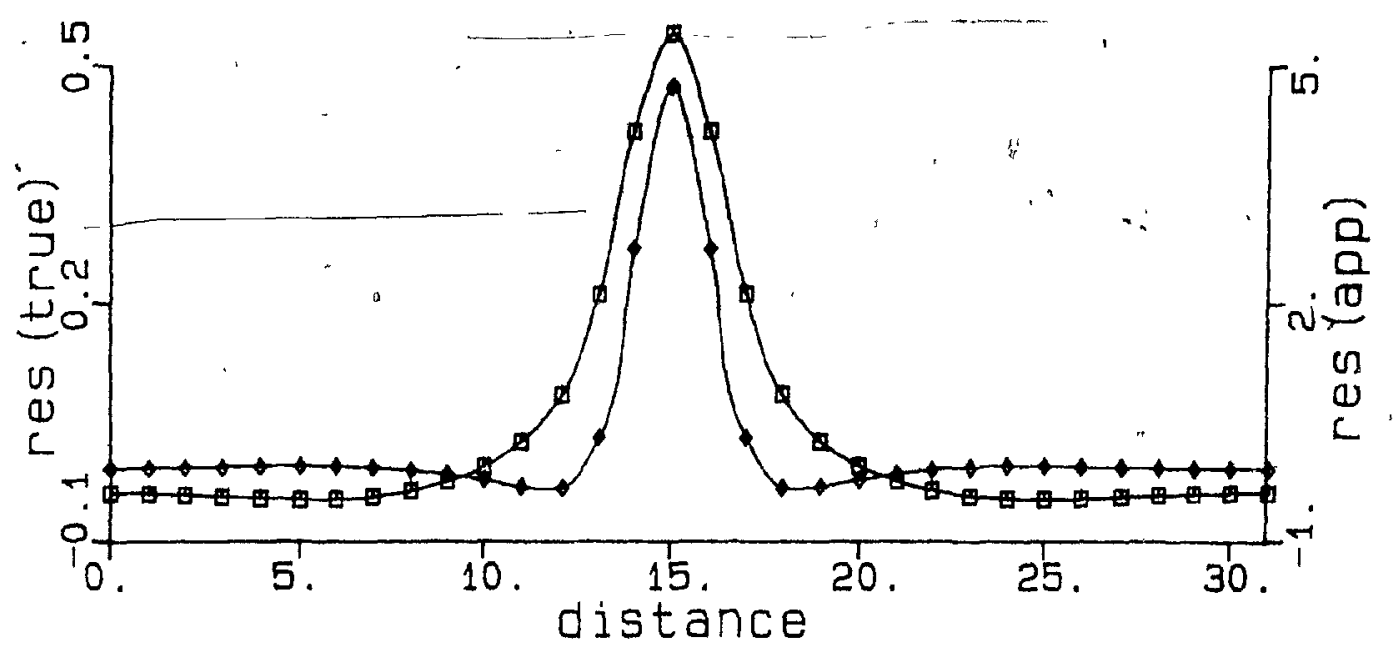

Pigure 3.5.1 True and approximate resolving kernels for topography estimates at distances 9 and 15 units. 


$$
R_{0}=\frac{E^{\star} \frac{\Lambda^{2}}{\Lambda^{2}+\theta}}{-E}
$$

that is, the resolving kernels of the initial parameter estimates $(h(r)=0)$. However $R_{0}$ is computed using values of depth and magnetization appropriate to the feature of interest, that is, the depth used in (3.8) is the average over the area to be analysed. Unfortunately $R_{0}$ assumes $a$ flat topography so the interfering effects of adjacent relief is not taken into account, contrary to $R(a p p)$. This inadequacy does not seem to effect the resulting resolving width estimates too severely for test examples. Figure 3.5.2 compares $R_{0}$ and $R$ (true) for the same parameters in Figure 3.5.1; the widths compare favourably.

\section{$\underline{3} \cdot \underline{6}$ Two layer inversion}

The multilayer topography inversion problem (MTI) as posed is severely underdetermined. As more layers are included in the model, the dimension of the parameter space is increased. Bounding the region of acceptable models to a manageable set becomes a much more difficult task. In the case of the two layer problem, given that an acceptable solution has been found, by analogy with the one layer case an infinite number of $z_{0}, J$ pairs $c a n$ be found independently for each layer that still satisfy the data. This section 
(a)

161

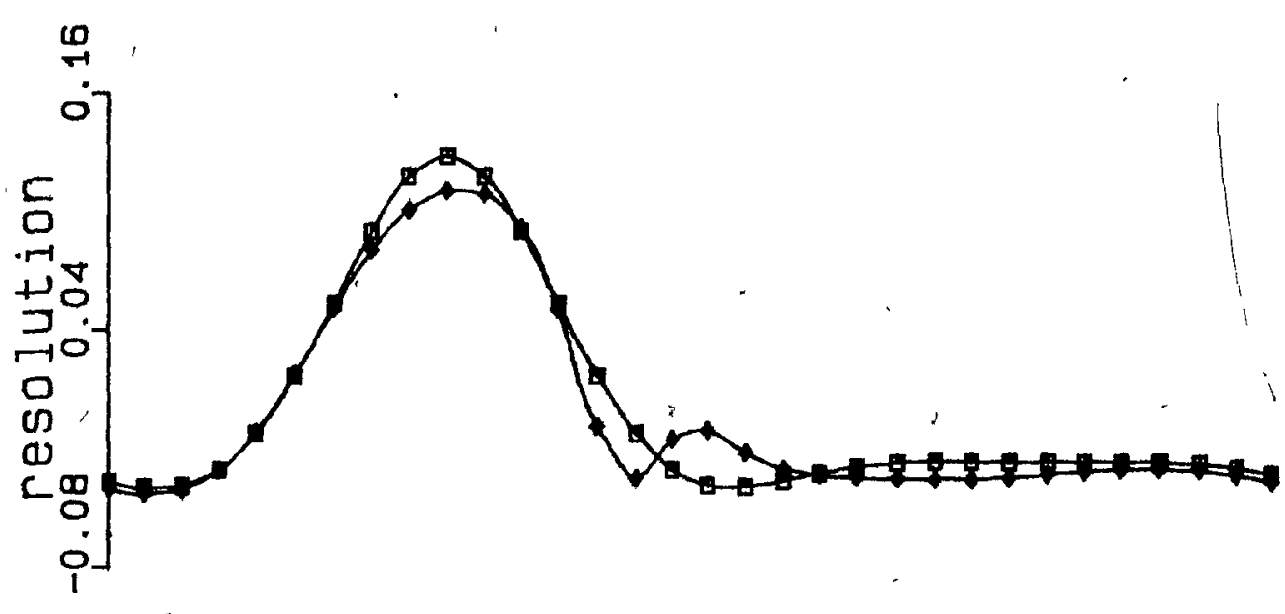

t approximate $\quad$ true

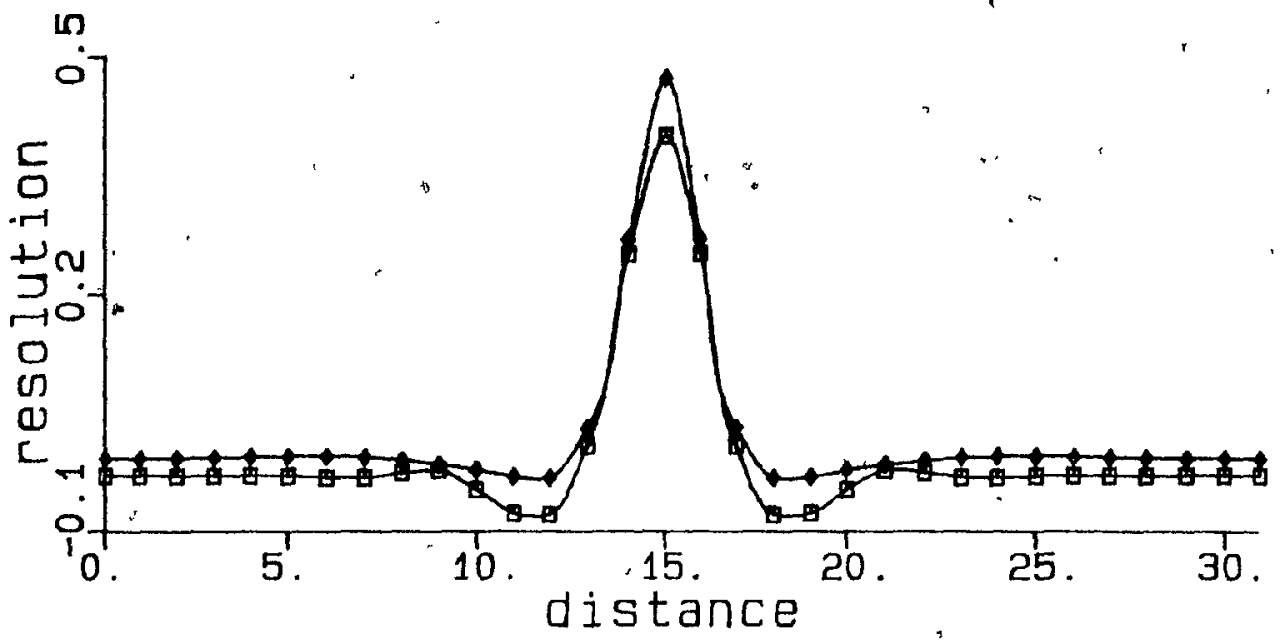

Figure 3.5.2 True and approximate resolving kernels for topography estimates at distances 9 and 15 units.

1 
presents examples of two layer inversions and attempts to explain the results in terms of the reparameterization imparted by the general n-layer singular value decomposition of section $(2.7)$.

The algorithm used is that of the one layer problem: the generalized inverse (equation (2.7.2.8)) is used throughout the iterative procedure. The number of free parameters is increased by the presence of the second layer, however in the absence of independent infomation, the spectral approach of section (3.2.2) is appropriate for the estimation of layer depths and magnetizations. Similarly, the a priori method of determining the appropriate damping factor (section (3.2.1)) can be applied to the two layer case by simply considering the inverse eigenvalue spectrum for each layer exclusively. From (2.7.2.8) these are given by:

$$
\begin{aligned}
& \Lambda_{1}^{-1}=F /\left(F^{2}+G^{2}\right) \\
& \Lambda_{2}^{-1}=G /\left(F^{2}+G^{2}\right)
\end{aligned}
$$

where $F$ and $G$ are the diagonal eigenvalue matrices for the corresponding one layer case. Typical spectra for layerl (top) and layer2 (bottom) are shown in Figures 3.6 .1 and 3.6.2, respectively, for a bottom layer magnetization twice that of the top. As mentioned previously, the reparameterization for the top layer is essentially a high-pass filtering, hence the spectra increase * with 


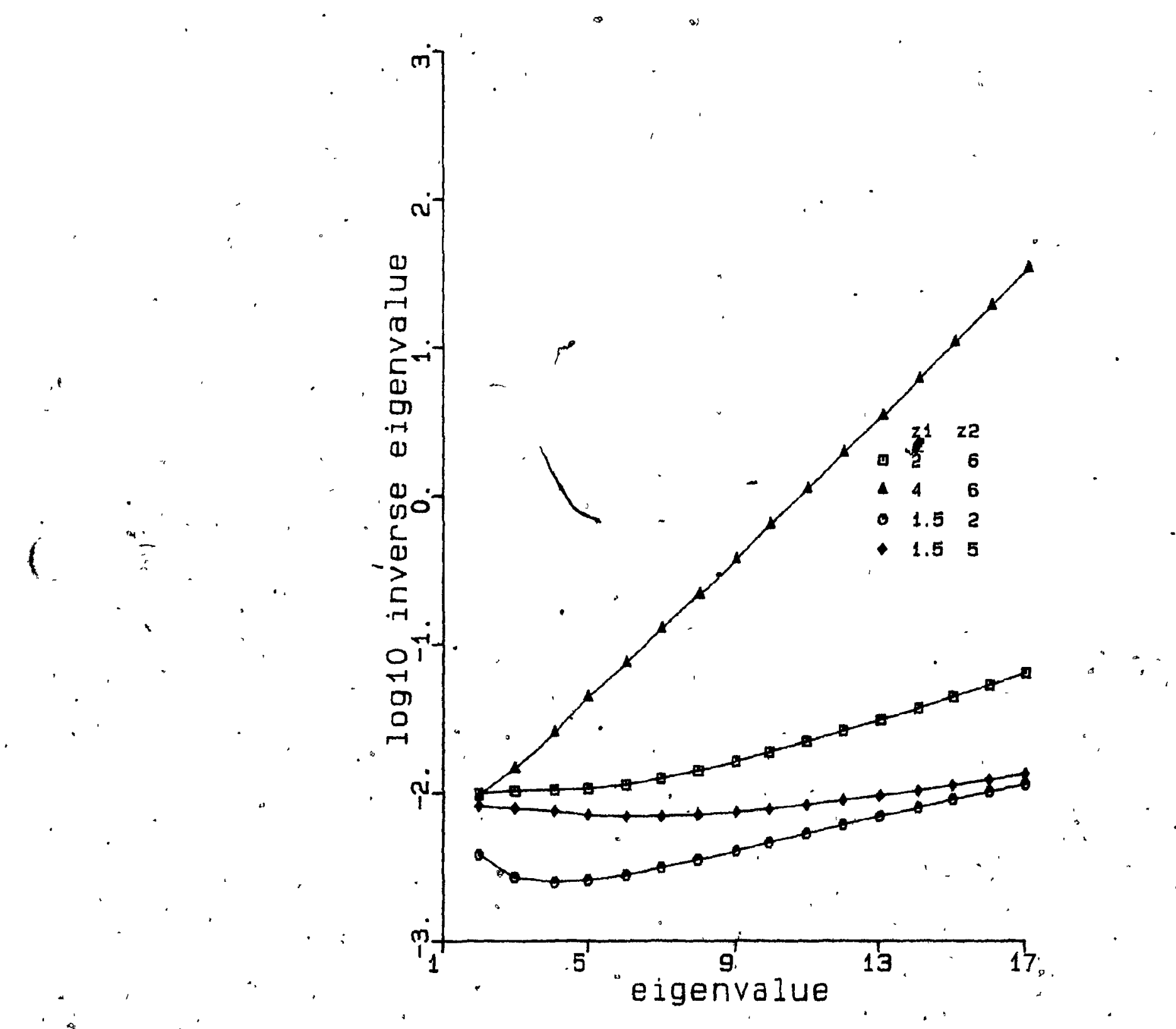

IIgure 3.6.1 Upper layer inverse eigenvalue spectrá (equation(3.9a)) for varying layer, configurations. Magnetization is $0.6 \mathrm{~A} / \mathrm{m}$ for top layer and $1.2 \mathrm{~A} / \mathrm{m}$ for bottom. 


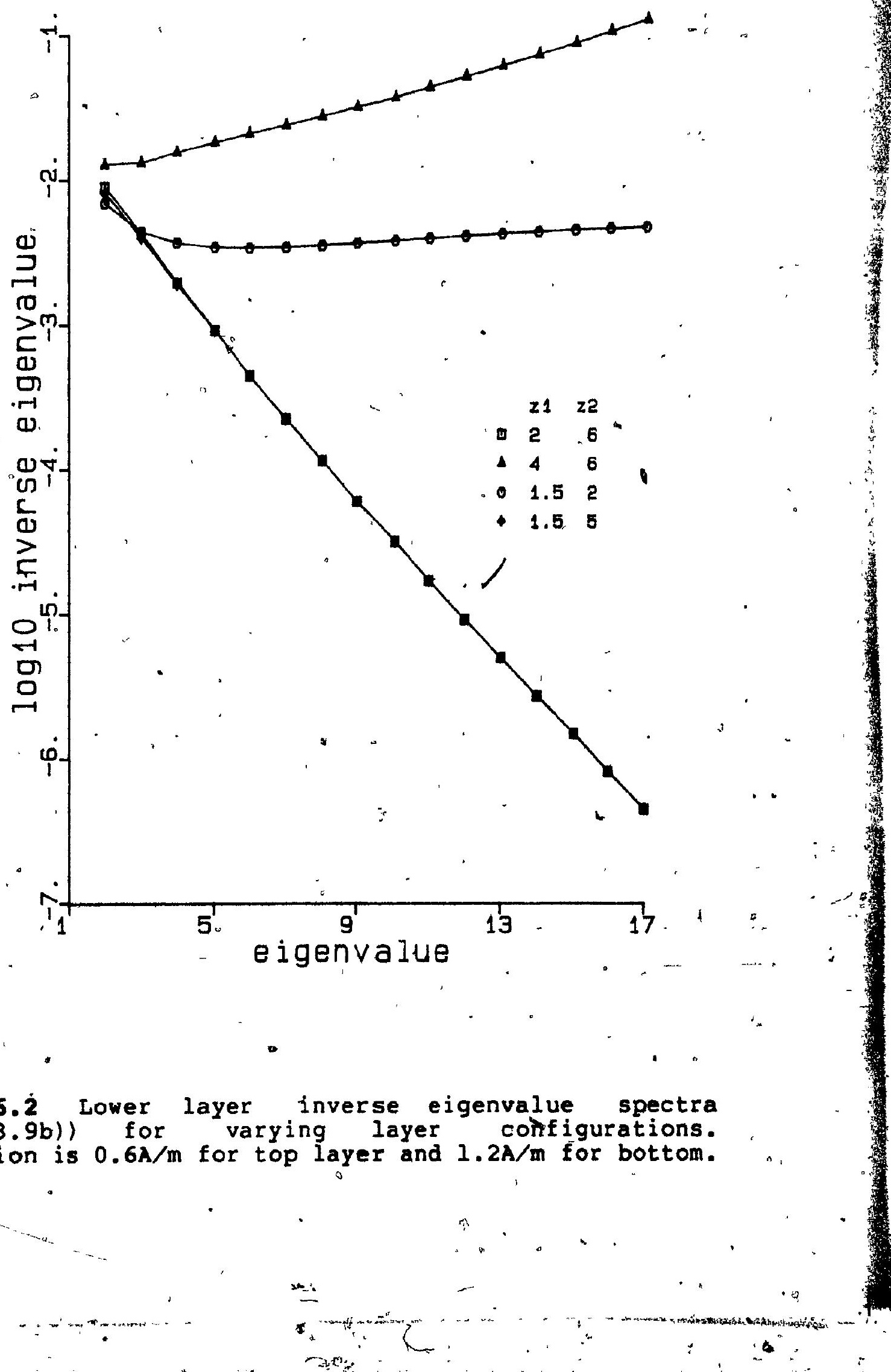


wavenumber as in the one layer problem. Inverse ieigenvalues for the bottom layer (Figure 3.6.2) are generally.well behaved (decreasing at high wavenumber) unless the layer separation becomes small. 'Consequently the estimation of a damping 'factor is mostly dependent on a priori bounds set for the top layer topography.

The results of inverting data from the two layer model LLI (Figure 3.6:3) with the correct values of layer depth. and magnetization are shown in Figure 3.6 .4 . In comparison with the' model (also shown), inversion has produced a severely attenuated and predominantly long wavelength lower layer topography and an upper layer that matches the true solution quite well except for topography components obviously originating from the lower layer. It is apparant that somesort of partitioning of wavenumber components between the" two layers has occured. Before discussing this in detail, it is instructive to compare the method with a fully nonlinear approach using derivatives calculated at each iteration based on a numerically determined singular value decomposition. For the latter technique, the"solution is not constrained to be formed from a linear combination of. the columns of the Jacobian (evaluated at a flat starting model, $h(r)=0)$. Tests have shown that the two methods" produce nearly identical results (not distinguishable on the scale of the figures) for flat starting models. Thus the adoption of a constant Jacobian does not invalidate the 


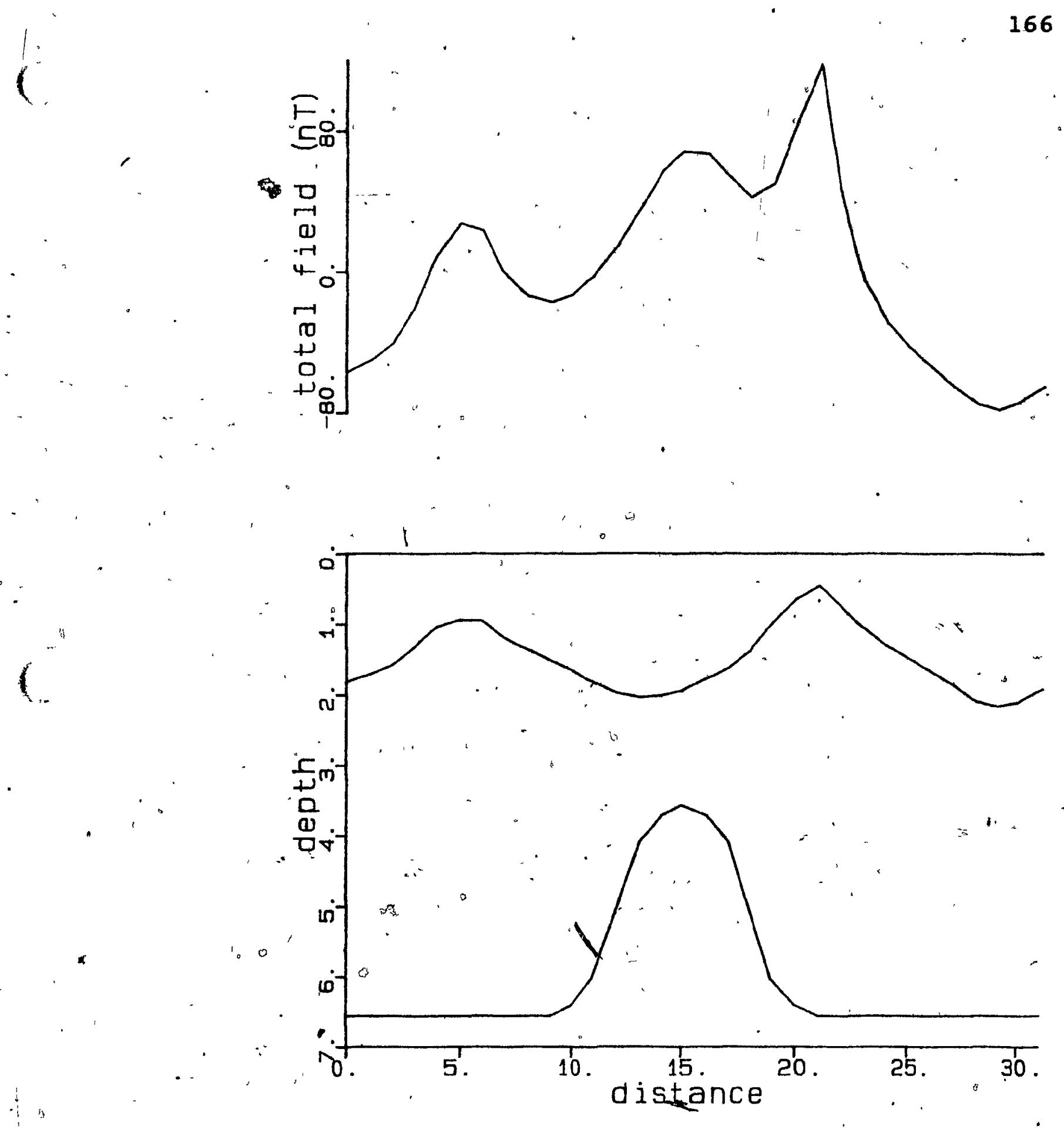



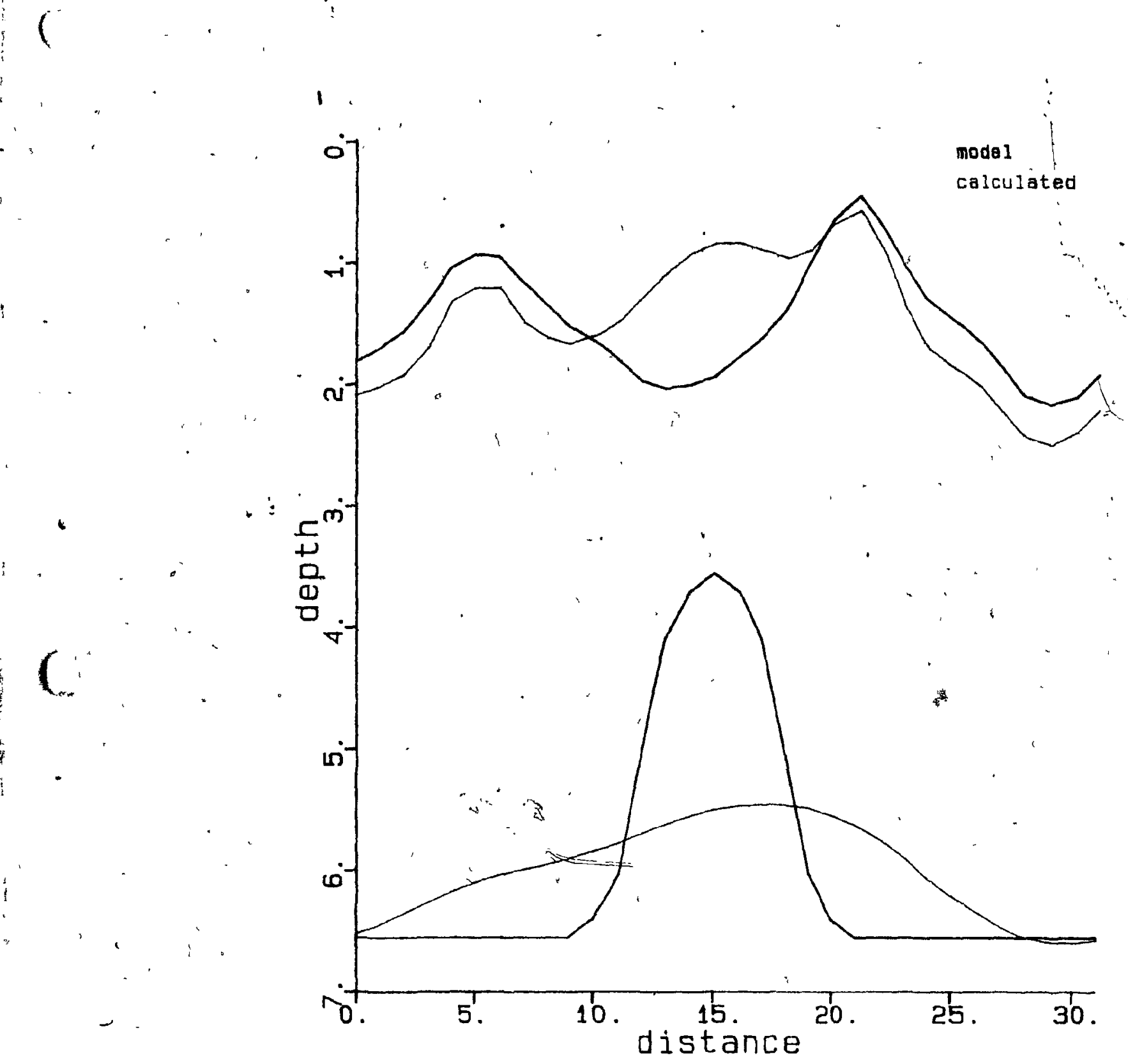
proposed method. The equivalence of the two approaches is expected from experience with the one layer problem wherein the FLAT produces a good approximation to the final solution. If the number of iterations is taken as an indication of the linearity of the problem, then the two layer problem is comparable to the one layer one; all test examples required less than 10 iterations.

In order to determine the effect of different starting models on the final solution and to evaluate the effectiveness of a constant Jacobian in areas of parameter space far from a flat starting model, model LLl was inverted with startng models in Figures 3.6 .5 and 3.6 .6 . The results. along with those from a nonlinear inverse programme are shown in the same figure. Using a Jacobian reevaluated at each iteraton (curves SVD in figures) causes some minor discrepancy with the proposed method (curves TOPO) but not large enough to invalidate its results. The difference between the two approaches arises from $(2.7 .2 .8)$ being a poorer approximaton to the true SVD at points in parameter space far from the flat layered model at which (2.7.2.8) is correct.

Figures 3.6 .5 and 3.6 .6 illustrate a fundamental problem encountered with multilayer inversion: the inability to resolve parameter, combinations from the lower layers. Inversions were also carried out with starting models consisting of a flat lower layer and various upper layers: 


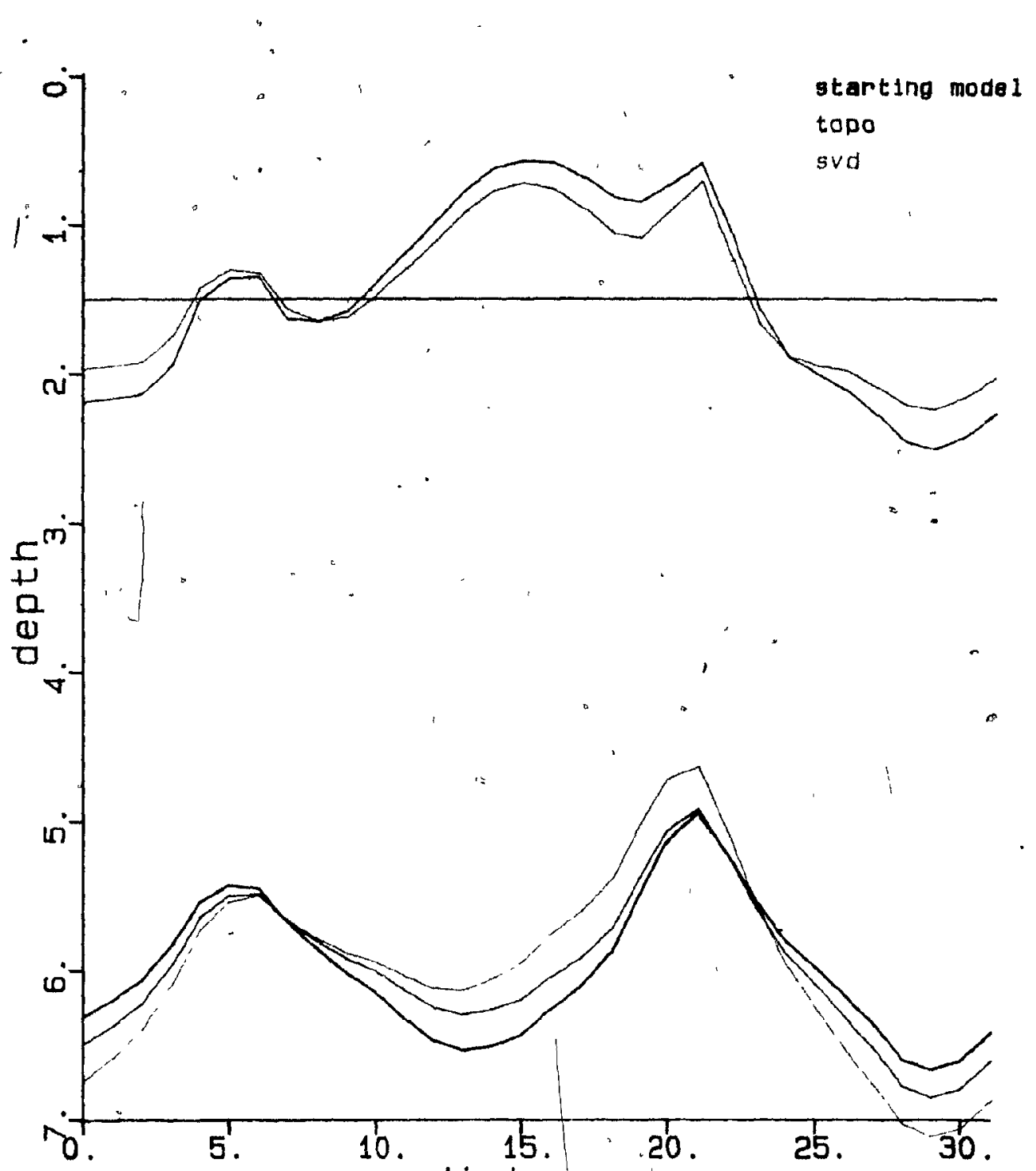

\section{distance}

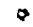

Figure 3.6.5 Starting models and results from the proposed method (curve TOPO) and a generalized nonlinear inversion programme (curve SVD) for model LLl data. 


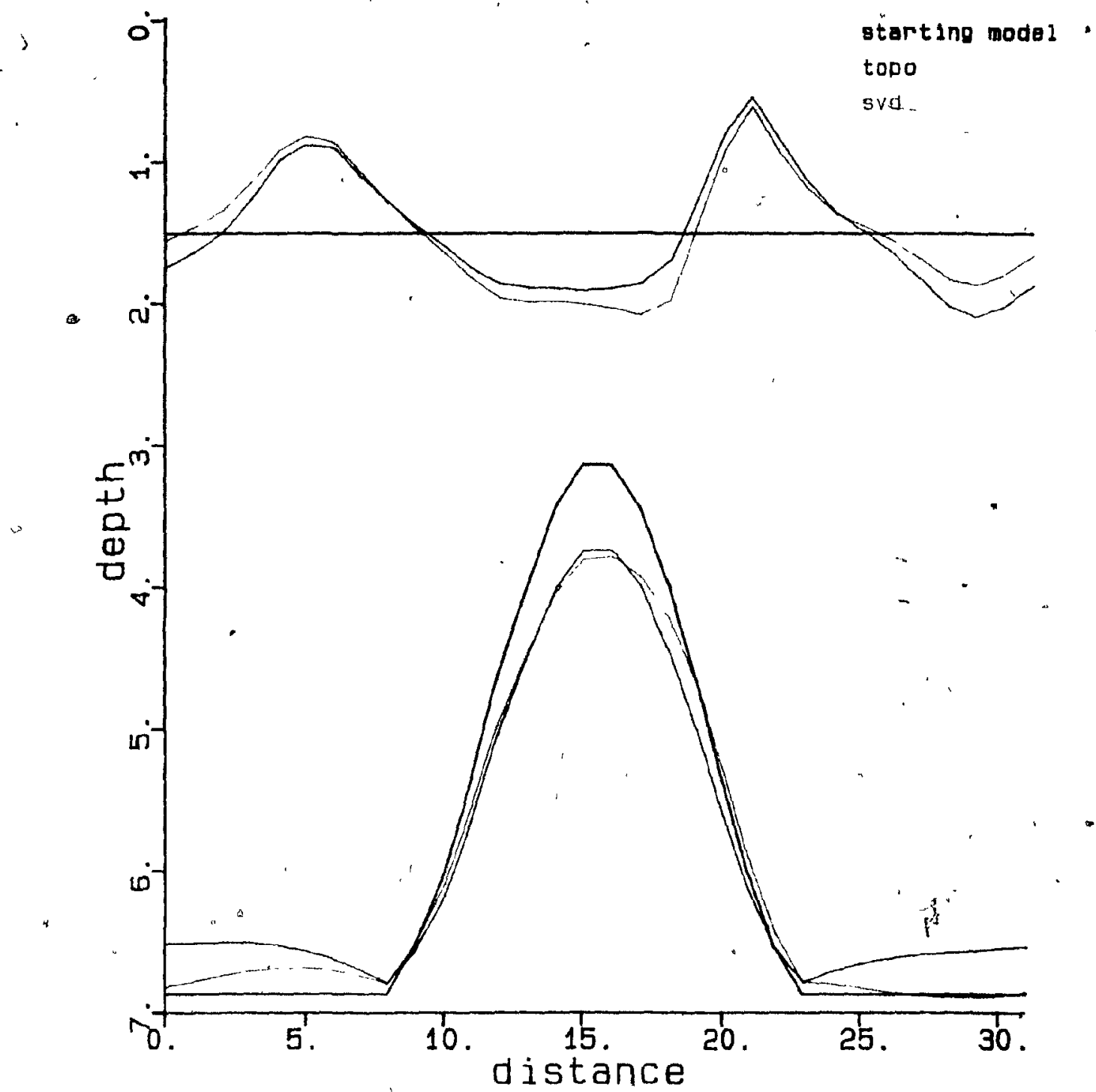

Figure 3.6.6 Starting models and results from the proposed method (curve TOPO) and a generalized nonlinear inversion programme (curve SVD) for model LLl data. 
these all produced results similar to Figure 3.6.3. The initial estimates offFigures 3.6 .5 and 3.6 .6 have changed little, the majority of parameter corrections being attributable to the upper layer. The lower layer then contributes little to data combinations associated with largeweigenvalues. Consequently Figure 3.6 .6 produces the best reproduction of the true topography due to the goodness of the lower layer starting model. Explanation of such effects is given by the reparameterization of Figure 2.7 where the longer wavelengths are partitioned to the lower layer and the shorter ones to the upper layer. The multilayer decomposition contains intrinsic constraints: the amplitude spectrum for each layer is fitted as closely as possible to that of a random distribution of dipoles occuring at the average layer depth. This was shown by the analogy to wiener filtering (section (2.7.2)). The criterion for partitioning the wavenumbers of the interfaces between upper and lower layers is based on the relative average depths of the interfaces. How well the true spectra match the 'random' ones will govern the accuracy of the solutions. As noted in section (3.2.2), this kind of spectrum is observed in the case of small amplitude, high wavenumber relief variations. Constraining the spectra of each layer to fit those of other models will produce correspondingly different results.

It is easy to concelve a situation with a 'high 
2

wavenumber' lower layer and a "Low wavenumber' upper layer, in which case the method will produce poor results. When the situation is reversed, the restriction of low wavenumber components to the lower layer is more appropriate to the parameterization and a better result is expected. Model LL2 (Figure 3.6 .7 ) represents such a relief; the results of inverting LL2 data are shown in comparison with the true model in Figure 3.6.8. It is apparent that in this particular model even some of the high wavenumber components of the top layer have been incorporated into the lower layer solution. Up to now, the modelled topographies have been uncorrelated as a function of depth. It is realistic to consider structures such as faulted and folded strata which give $r$ ise to correlations between layers. The results from inversion of a foldea two layer model (LL3, Figure 3.6.9)

- where the two reliefs are identical are shown in Figure 3.6.10. Inversion has recovered the major features of both layers, but significant attenuation has occured in "the lower layer. Figure 3.6 .10 also shows the results of 'inverting noisy LL3 data (the noise sample of Figure 3.3 .2 was added to the response of model LL3). The RMS error for the noisy solution is less than $1 \mathrm{NT}^{2}$ and as expected the majority of the noise has been modelled by the upper layer."

The two layer inversion method can be applied to the case of a single uniformly magnetized layer for which the upper and lower boundaries are to be mapped. Figure 3.6.11 
173
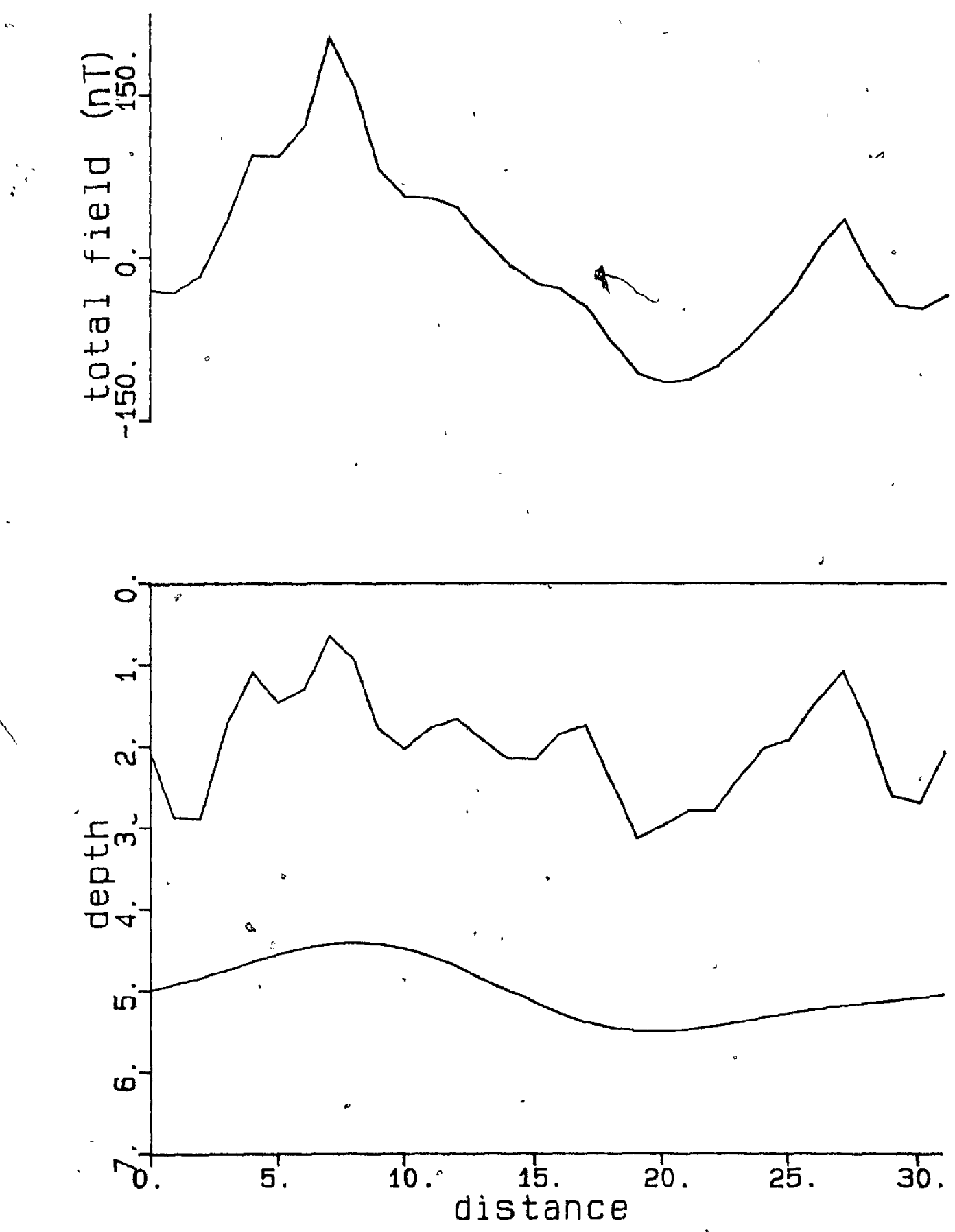

- Figure 3.6.7 Model LL2. Lower layer magnetization is 3.0 $\mathrm{A} / \mathrm{m}$, upper layer magnetization is $0.6 \mathrm{~A} / \mathrm{m}$. 
(

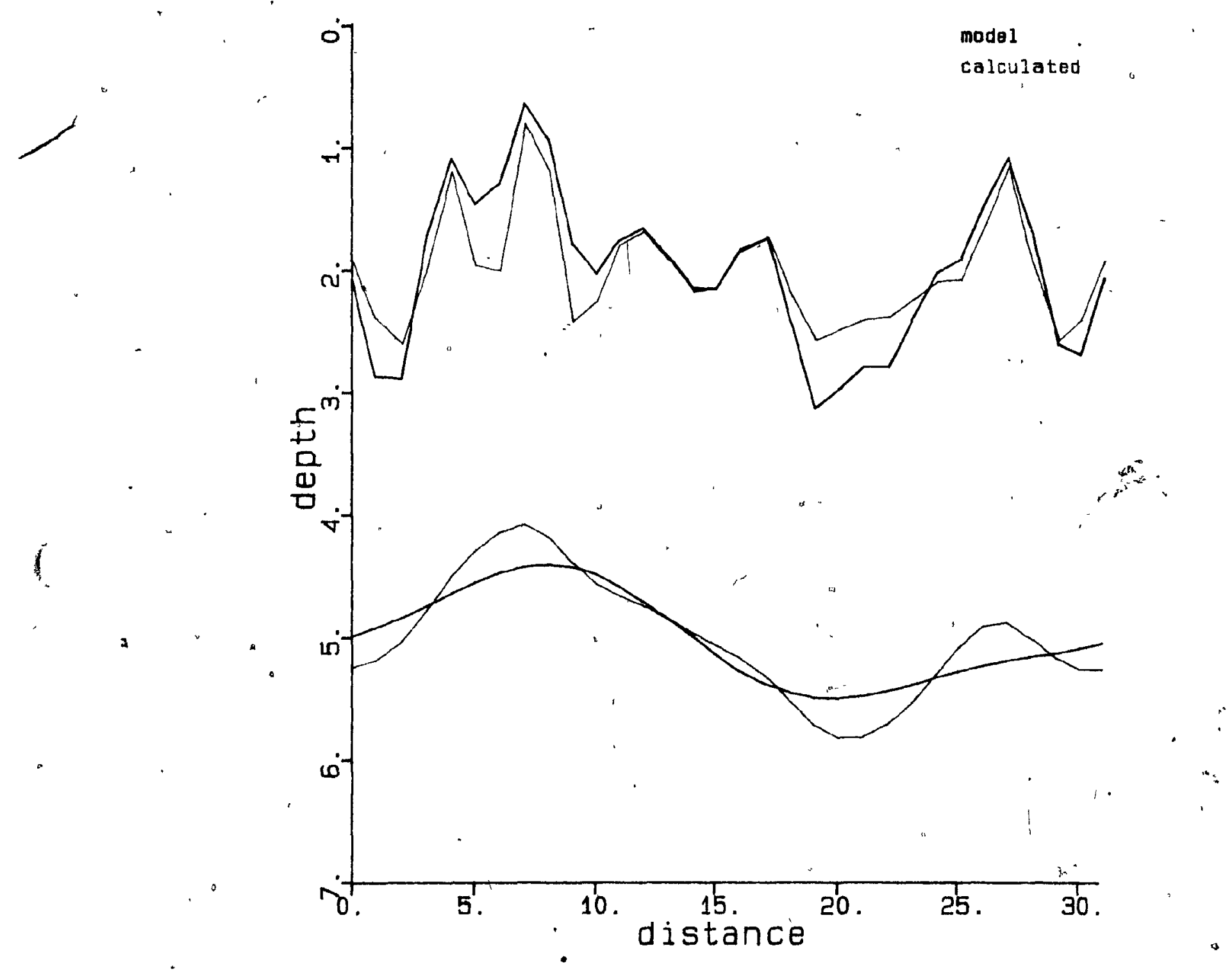

Figure 3.6.8 Model LL2 and calculated solution. 


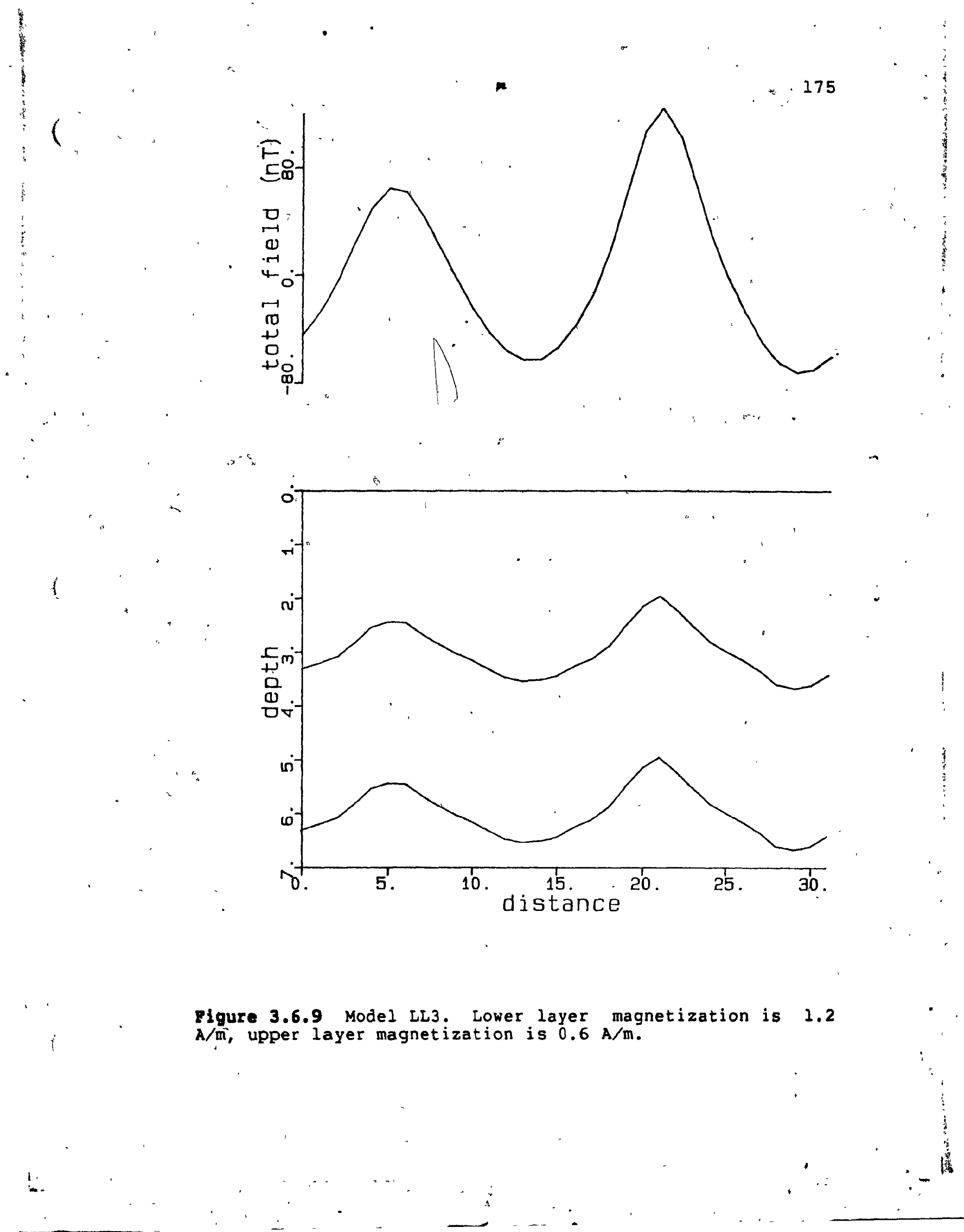




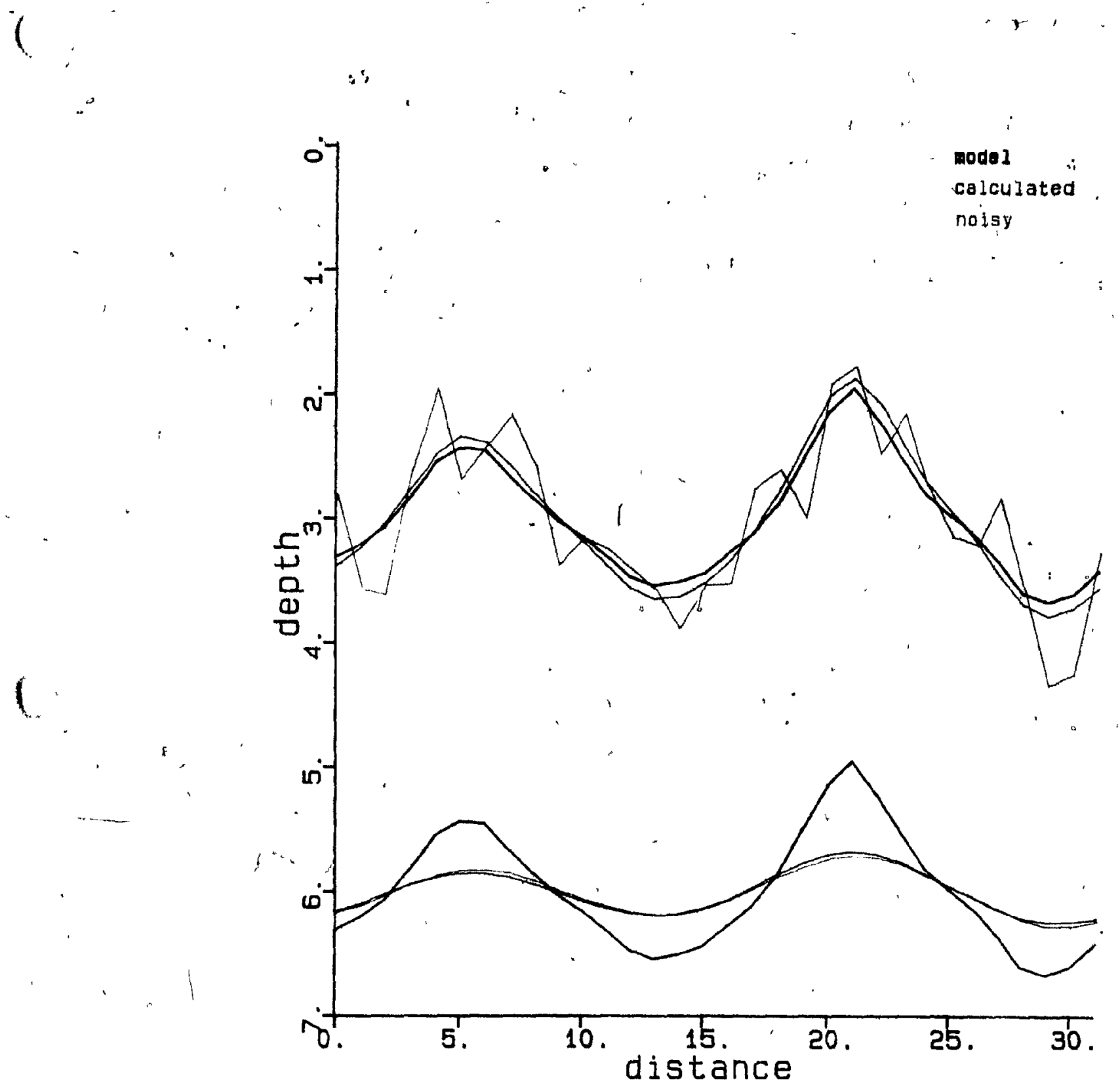

Figure 3.6.70. Model LL3 and calculated solutions for noisy and noise-free data. 
177
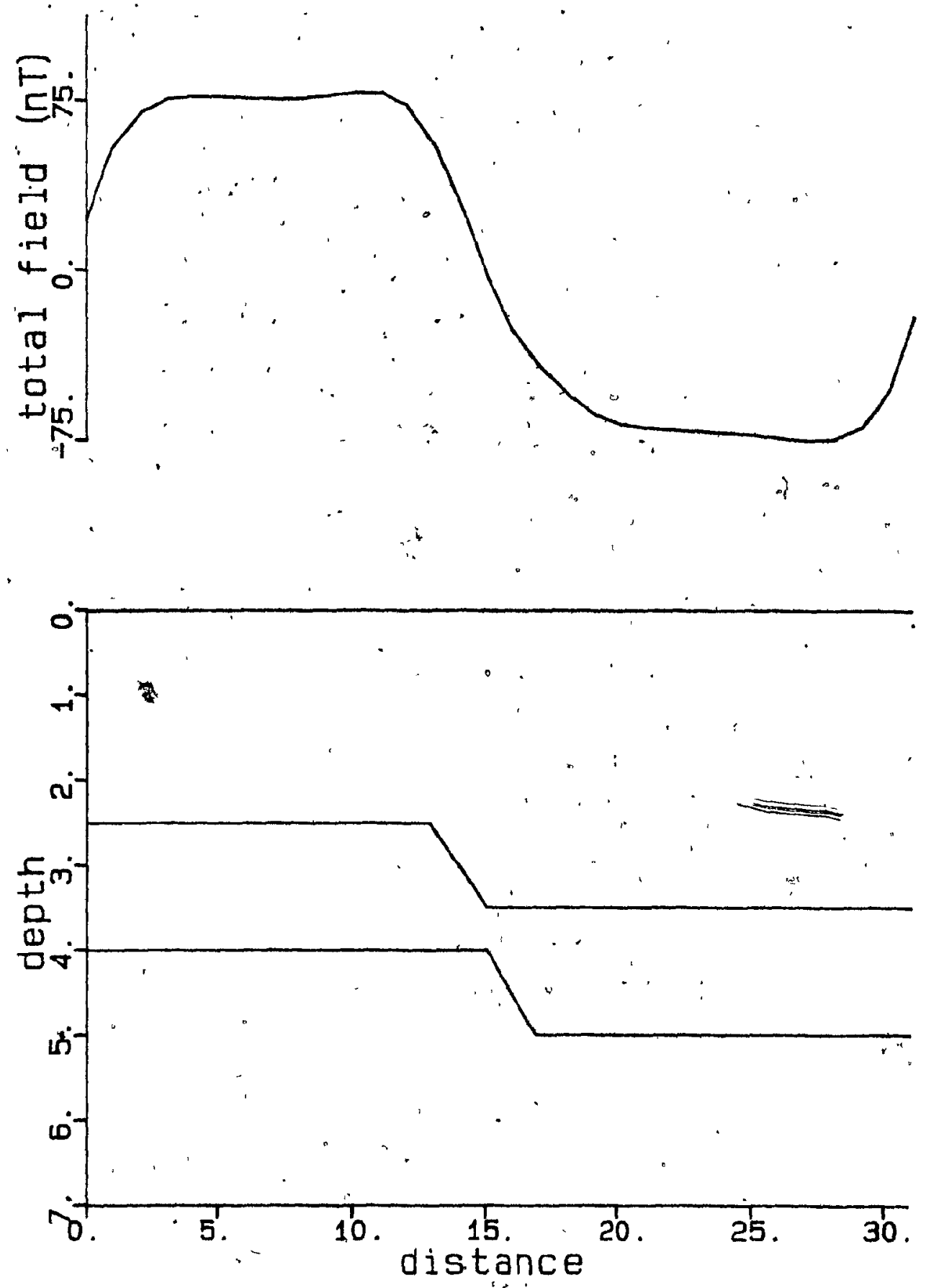

Figure 3.6.11 Model LL4. Lower layer magnetization is 1.2 Adm, upper layer magnetization is $1.2 \mathrm{~A} / \mathrm{m}$. 
presents such. a laỵer (model LL4) which has been faulted. Inverting model: "LLA data," as shown in Figure 3.6.12, has produced a good approximation to the true structure, mainly' because the layer separation is small, thus producing less disparity between each. layer's parameterizations. Discrepancies at the profile edges,are due to the effects of fircular convolution assumed by the. Fast Fourier transform. This effect can be reduced by the application of a sutable window function to the data.

- The non-uniqueness of the multi-layer problem "is incfeassed due to the existence, of "solutions to the homogeneous prablem:"

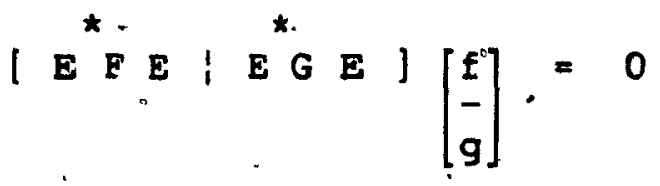

Equation 3.10 is the linearized forward problem for two layers; the inclusion of higher order terms from the expansion (2.6.2.4) results in the nonlinear from of $(3.10)$ which is easily solved by the two-layer inversion method of section (2.7) applied with all the data set to zero, The simplest solution to the homogeneous problem is obviously the case of $f(r)=0$ and $g(r)=0$. This results if the inversion is initialised with flat models; starting the procedure with non-zero reliefs results in topographies that produce no observed field. Figures 3.6 .13 and 3.6 .14 show examples of such solutions for the case of a magnetized layer occuring 


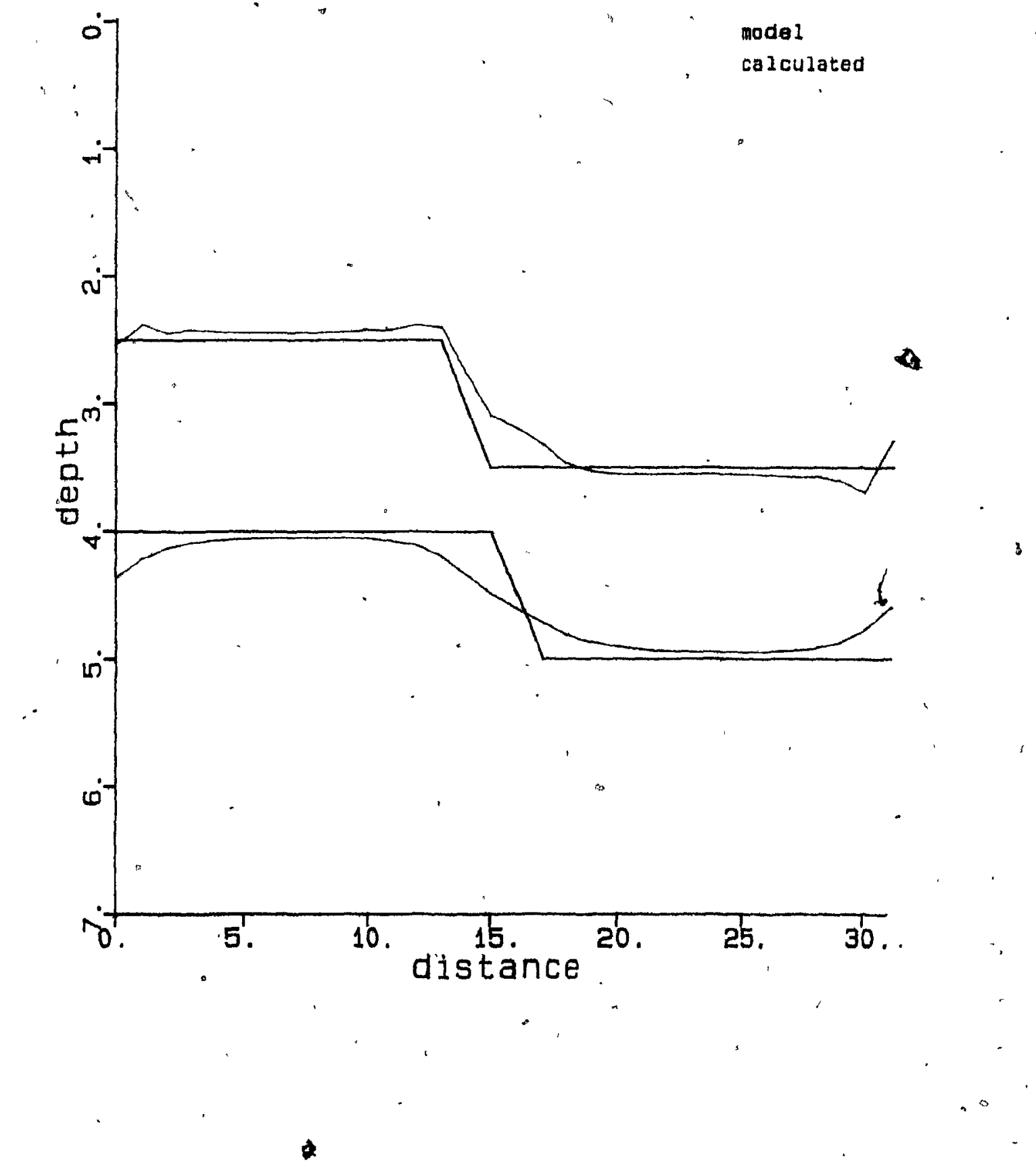

Figure 3.6.12 Model LL4 and calculated solution: 

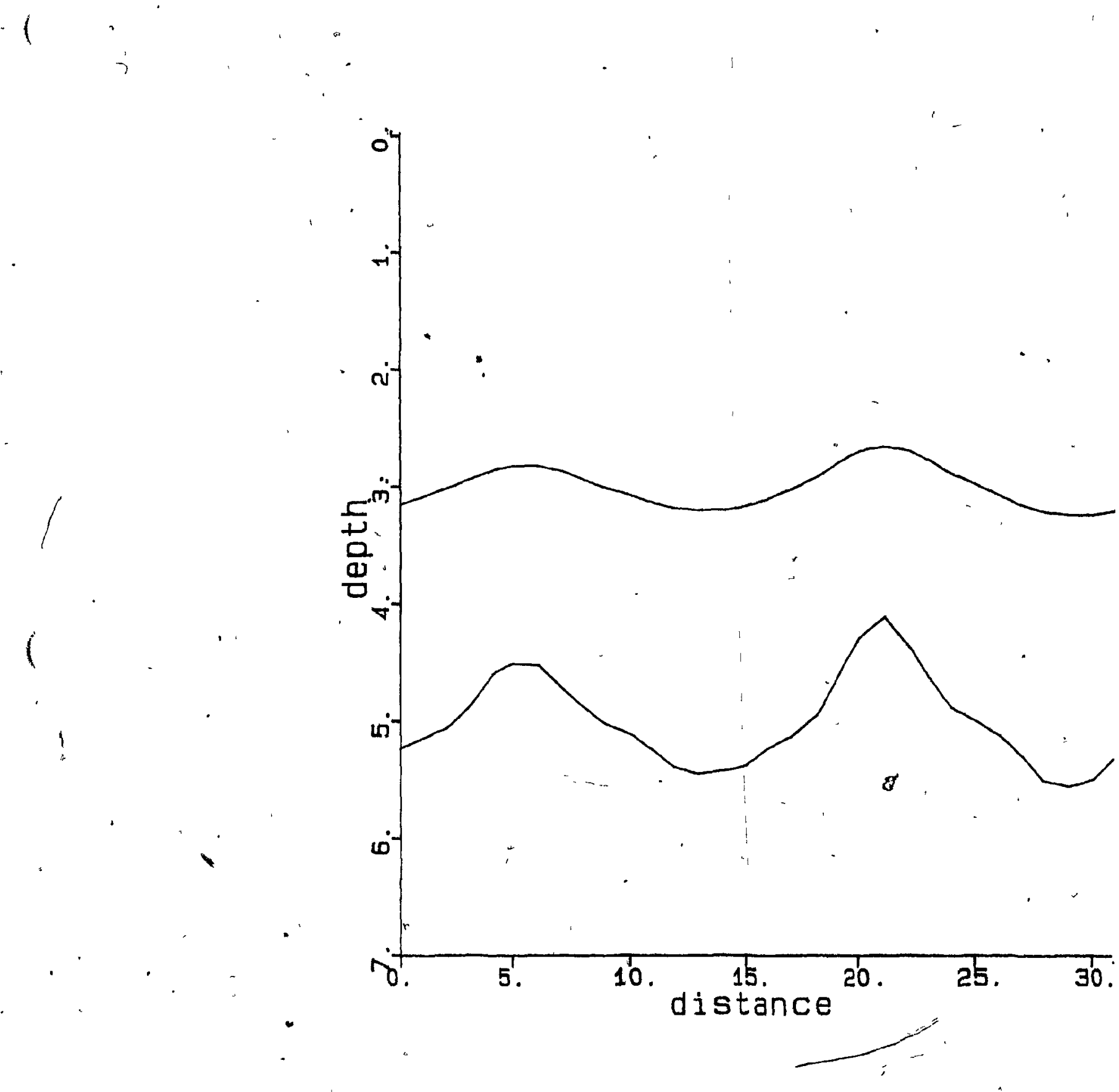

Figure 3.6.13 A solution to the homogeneous problem (nonlinear form of $(3.10)$ ). Average layer depths are 2.0 and 5.0 units. Magnetization contrasts are $1.2 \mathrm{~A} / \mathrm{m}$ and $-1.2 \mathrm{~A} / \mathrm{m}$ for the upper and lower interfaces. 

in a non-magnetic medium: these solutions are dependent on the starting model and are not unique. Surprisingly, both figures show that such a layer is not detectable even if it exhibits strong relief.

\section{3: 7 Constrained inversion}

In section (2.8) the introduction of equality constraints for any number of parameters applicable' to both the one ard two layer cases was outlined. The addition of such information to the problem, primarily reduces the non-uniqueness in the final solution estimates. Results for the one layer problem (sections (3.2) and (3.3)), suggest that obviously a high degree of confidence can be placed in the final solution if the layer depth and magnetization are specified correctly. It is of more, interest to note that. the non-uniqueness of the solutions is restricted to 'the high wavenumber components of the topography. By varying the damping factor and initializing the algorithm, with different models, the resulting class of solutions differ only in their smoothness and not ${ }^{\star}$ in terms of the basic structure of the computed relief.' Consequently; "it is expected that equality constraints for the one layer case will be. of use primarily in checking the validity of ,the depth and magnetization parameters and, more importantly, whether the single interface model is appropriate for a particular data 
set. Figure 3.7 .1 shows the results of inverting the $L 2$ data subject to the constrained parameter having the value indicated by the arrow in the figure. For comparison, the unconstrained solution is also shown. The constrained solution is acceptable at the $1 \mathrm{nT}^{2}$ level but smoother solutions could be obtained by varying the depth and/or magnetization values. Obviously when redefining auxiliary parameters does not produce an acceptable solution that satisfies the constraints the single interface model must be abandoned.

The above comments apply equally to the two layer problem, however the role of constraints becomes important in reducing the ambiguity between the numerous possible solutions caused by the non-unique nature of the wavenumber separation between the lâyers, as discussed in section (3.6). Contrary to the one layer problem, the non-uniqueness is not restricted to the high w wavenumer/ topography components. As an exampiey the two-layer constrained algorithm (equation $(2.8 .3 .6)$ ) has been applied to model LLI data (Figure 3.6.4) subject to the constraints on the lower layer topography (indicated by arrows in Figure 3.7.2). The three constrained values have been set to their correct value in an attempt to recover the true-solution. The constrained estimates of Figure 3.7 .2 shows that, inversion has successfully surpressed the central 'hump' in the top layer relief that "resulted from partitioning some of the 



\section{s

lower layer wavenumbers into the unconstrained top layer solution. Unfortunately, the lower (constrained) layer shows little improvement over the unconstrained results of Figure 3.6.3 apart from the constrained parameters. The random dipole distribution model (section (3.6)) dictated by the multi-layer inverse (equation $(2.7 .2 .10)$ ) is still not appropriate even with constraints for the given model: more detailed a priori spectral information is required for a more successful reparameterization and inversion. 


\section{CHAPTER 4}

\section{I Field examples}

\subsubsection{Introduction}

In this section, the feasibilty of the proposed method in mapping crustal interfaces is demonstrated for both, the gravity and magnetic cases. No attempt is made to provide a detailed interpretation of the actual data examples. as the aim of this thesis is the development of an interpretive technique.

\subsubsection{Abitibi area gravity}

The area chosen coincides roughly" with the "Abitibi 'greenstone belt, of Quebec and ontario and ranges from $48^{\circ}$ to $50^{\circ} \mathrm{N}$ and from $75^{\circ}$ to $81^{\circ} \mathrm{W}$. In order to reduce edge effects at grid borders, an extended grid from $47.5^{\circ}$ to $80.5^{\circ} \mathrm{N}$ and $74^{\circ}$ to $82^{\circ} \mathrm{W}$ was used in the computations. The data consisted of 6648 gravity measurements reduced to a common datum and

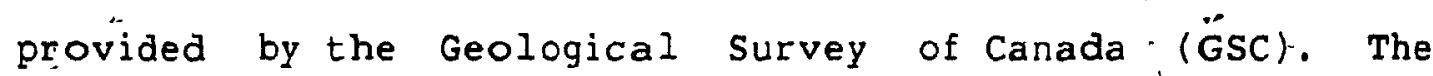
station density, although extremely variable, averages to approximately one stątion per $15 \mathrm{~km}^{2}$. A .bicubic spline interpolation programme (IMSL routine IQHSCV) was used to 
produce a $128 \times 256$ glid (grid interval approximately $3 \mathrm{~km}$ ) which was then resampled, after appropriate. low-pass filtering to reduce aliasing, resulting in a $64 \times 64$ rectangular grid with grid intervals of $9.6 \mathrm{~km}$ EW and $5.4 \mathrm{~km}$ 'NS: The resulting Bouguer anomaly mapo is given' in.Figure 4.1. Resampling has resulted in some smoothing of the original data, however for the purposes of this study, that is, determination of Moho topography, only the longer wavelengths of the gravity field are of interest. Additionally, the (larger) EW grid size is considered small enough to accurately represent any field components resulting from such a deep source.

The Abitibi greenstone belt is situated on the southeastern boundary of the Archean Superior Province and is an easterly trending tectonic unit consisting of regionally metamorphosed, interbedded mafic volcanics and sediments. For an overview of the geology of the area see Ludden and Hub ert $(1984)$. Figure 4.2 shows a simplified geological map of the Abitibi region.

The gravity field over the Abitibi shows a regional negative field of roughly $-50 \mathrm{mgals}$. Superposed are negative anomalies of similar areal extent with relatively high horizorital gradients at anomaly edges indicating a near surface source. These occur mainly in the central and northeastern part of the study area. Positive anomalies of generally smaller size occur in the south west and generally 


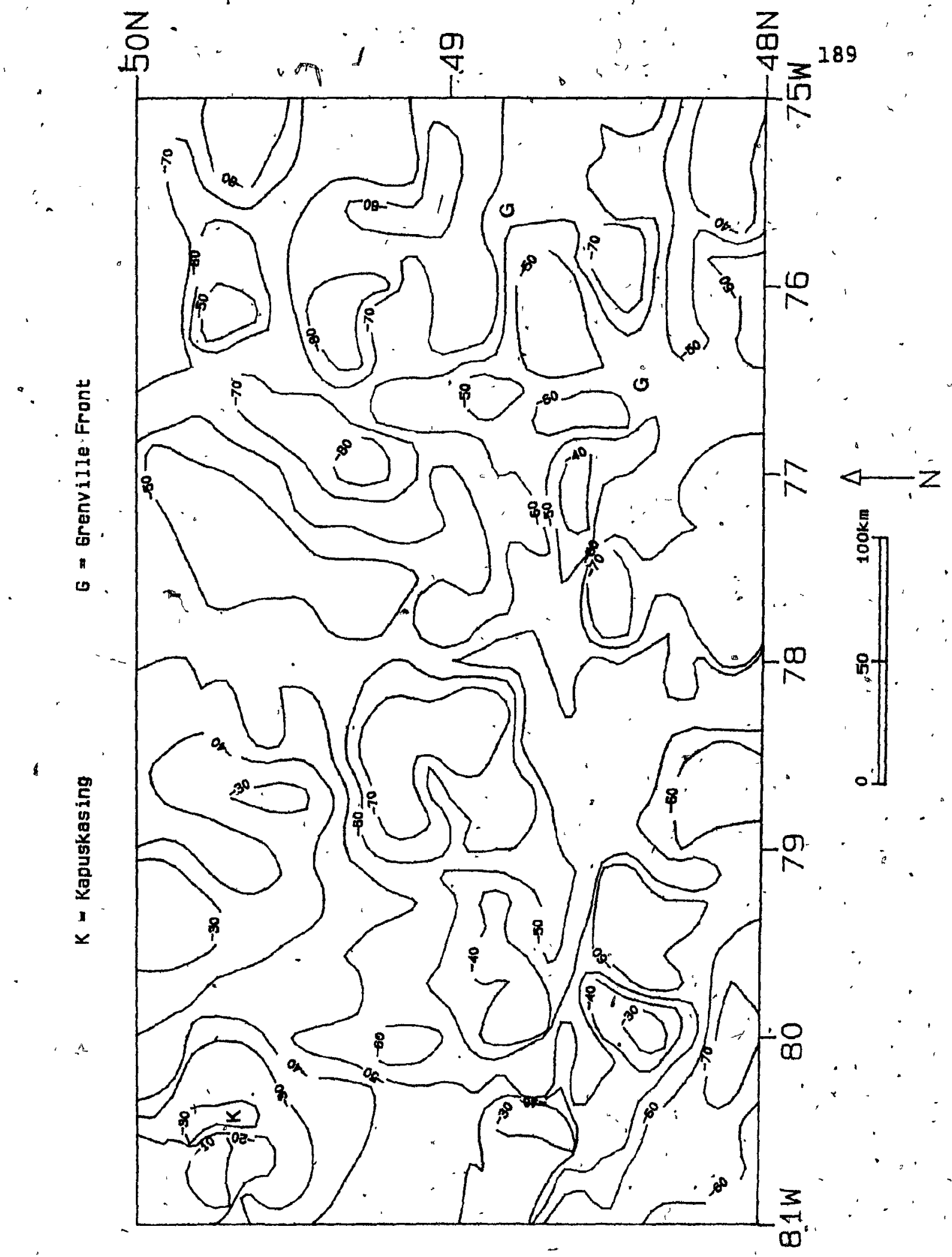

Figure 4.1 Bouguer gravity map of study area. Contour interval is 10 mgals. 


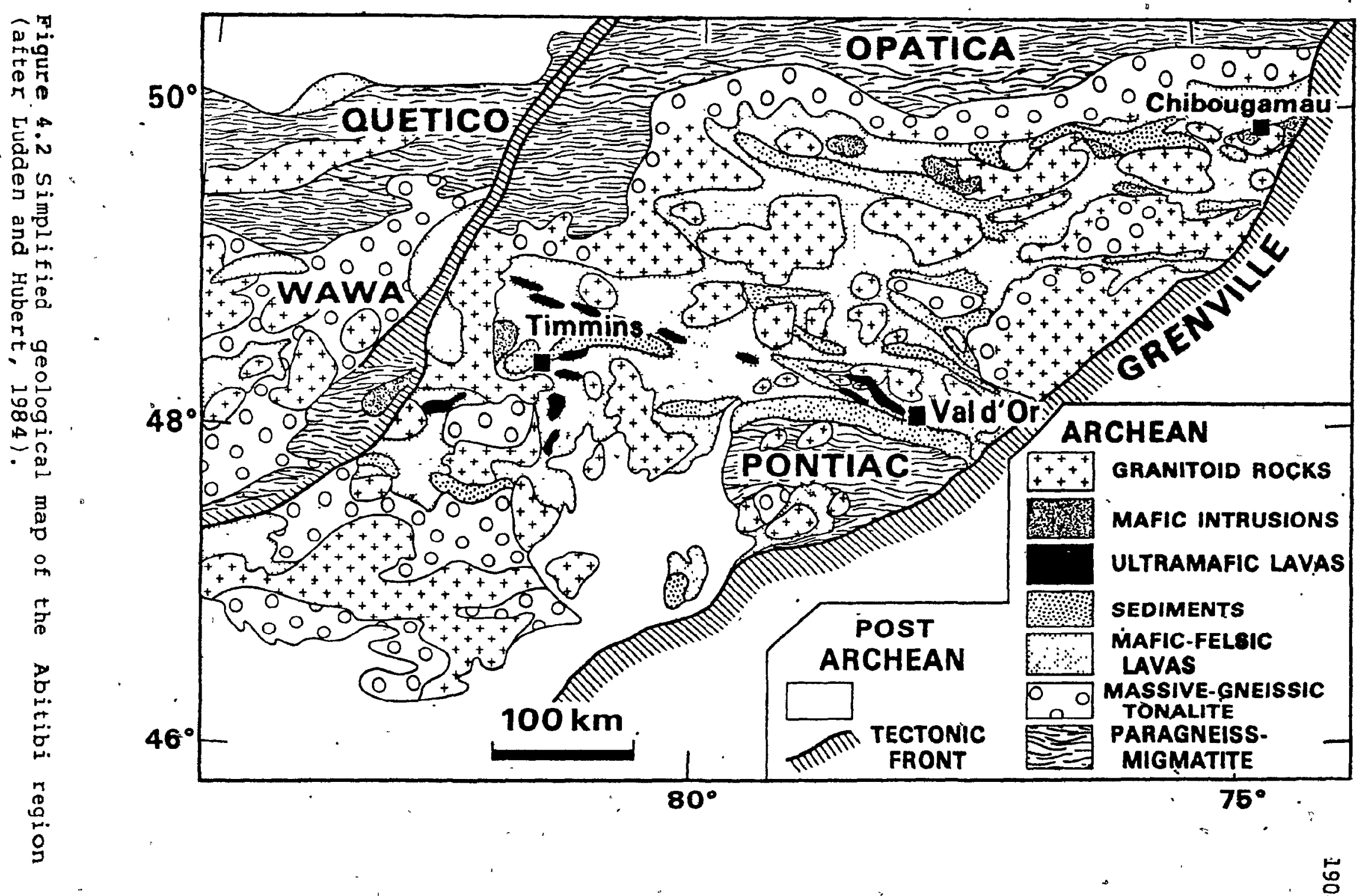


coincide with major mafic metavolcanic assemblages and mafic/ultramafic intrusives. The large gravity high in'the north west coincides with the adjoining Kapuskasing structural feature. No distinct change in character in the gravity field occurs over the Grenville front, although on larger-scale maps, the field tends to be much less variable within the Grenville Province. This may simply reflect a change in the variation of near surface lithology (Schwarz et al, 1984). Comparison of the geology with the Bouguer anomaty for. the study area reveals a correlation between the gravity lows and granite batholiths. Modelling of individual anomalies suggests the granite plutons have depth extents of up to $5 \mathrm{~km}$ (Schwarz et al, 1984).

For modelling purposes, a mean interface depth of $33 \mathrm{~km}$ and a constant density contrast of $0.5 \mathrm{~g} / \mathrm{cm}^{3}$ ere adopted. These parameters,correspond to the average depth of the Mohorovicic discontinuity and the average density contrast between the lower' crust and the upper mantle. It is realised of course that the observed data contain both the effects of lateral variation of the Moho and, also the effects' of anomalous density sources ranging in depth from the near: surface down to the upper mantle. However, it is expected that an estimate of the variation in Moho topography can be extracted from such data. Figure 4.3 shows the results of inversion with the computed relief measured relative to the average 'interface depth $(33 \mathrm{~km})$. It is apparent that the 


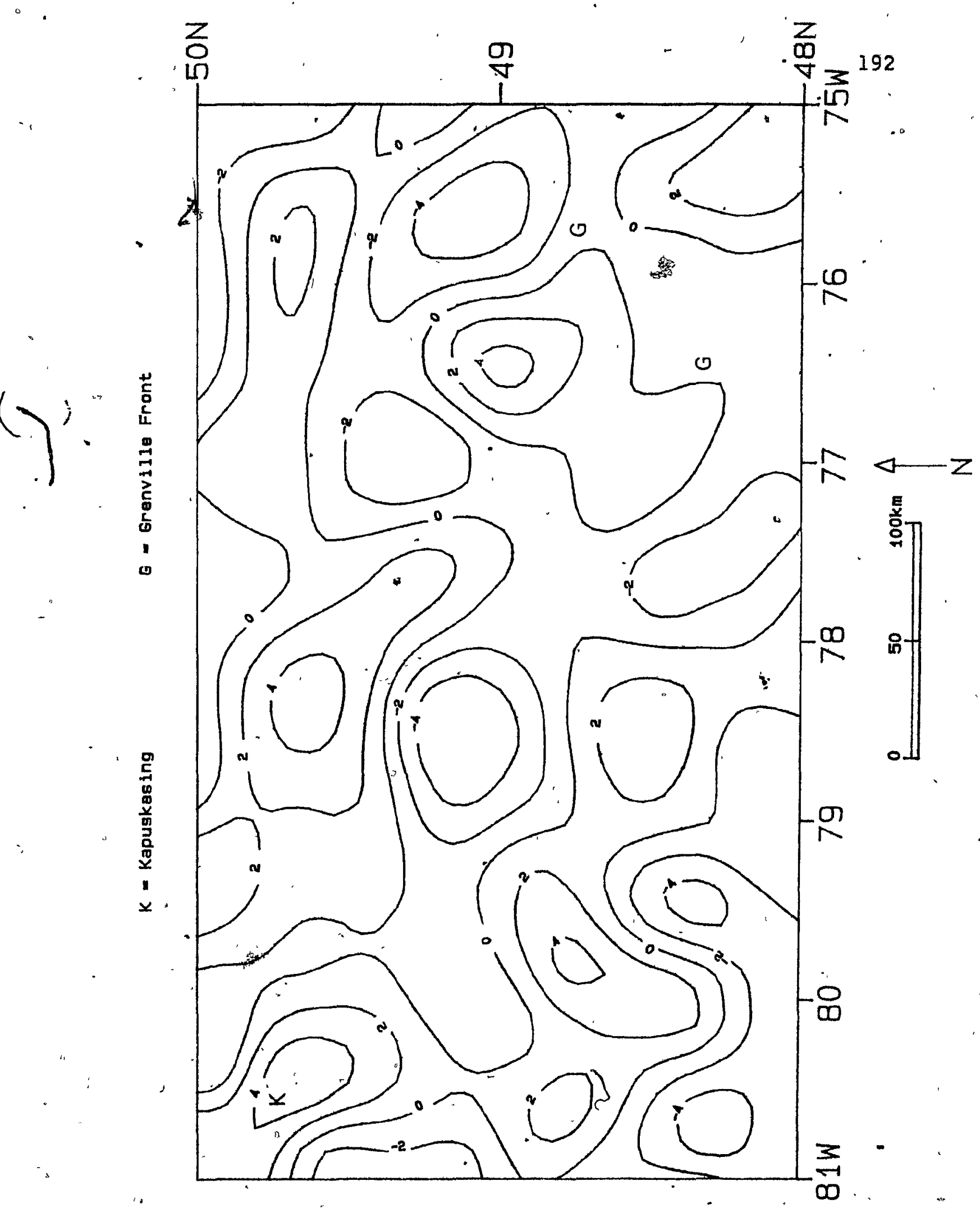

Figure 4.3 Calculated variation in Moho topography. Contour interval is $2 \mathrm{~km}$. 
granite lows in the centre of the map have been modelled as an increase in crustal thickness and effectively split the topography highs to the north and south. The Kapuskasing gravity high results in an isolated topography high with an associated low to the.south east. This is not however the preferred interpretation of this feature as it is believed that the gravity anomaly is due to an up-thrusted lsection of the lower crust (Percival and Card, 1983). Interestingly, the Grenville front corresponds to an area of little variation in relief while the Grenville Province to the south east is characterised by an elevated Moho for a thinner crust). Mereu and Jobidon (1971) suggest an increase in crustal thickness, for the Grenville Province based on seismic refraction studies on a line perpendicular to the Grenville front. The size of relief in Figure 4.3 is in accordance with that postulated by Schwarz, et al (1984) ibased on more conventional gravity modelling.

Figure 4.4 shows the field produced by the model of Figure 4.3 with respect to the average observed field (removed during inversion). The resulting field, due entirely to Moho topography, could be considered to be a first approximation to the regional gravity field over the Abitibi region. A broad decrease in a southerly direction in the computed field is noted which agrees with a gentle southerly dipping Moho as suggested by Parker (1984) on the basis of seismic refraction studies in the area. 


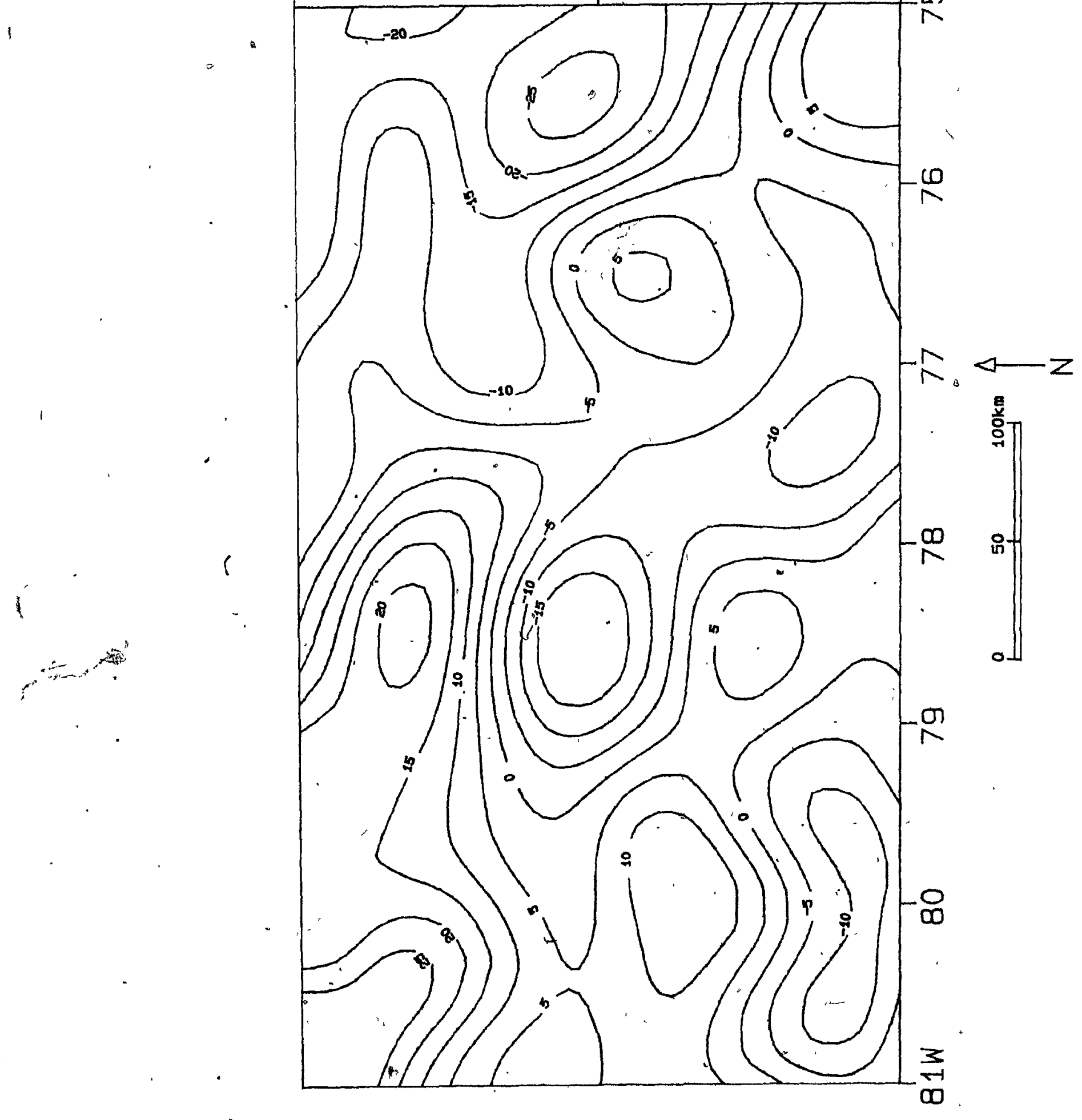

Figure 4.4 Gravity field produced by the model of Figure 4.3. Contour interval is $5 \mathrm{mgal}$. 


\subsubsection{Abitibi-Grenville magnetics}

The study area for the magnetic example is bounded by latitudes $48^{\circ}$ and $52^{\circ} \mathrm{N}$ and longitudes $72^{\circ}$ and $78^{\circ} \mathrm{W}$ and measures approximately $450 \times 450 \mathrm{~km}^{2}$. The region contains the boundary between the Grenville and Superior Provinces and includes the eastern part of the Abitibi belt. The aeromagnetic data (GSC map NMl.8) are total field values digitised from 1:63 360 contour maps produced by the GSC: they were made available on a square grid (grid interval $812.8 \mathrm{~m}$ ) centred on the flight lines of the original maps. The IGRF (International Geomagnetic Reference Field) has been subtracted yielding a residual field represented by over 300000 points within the area." A $500 \times 500$ grid was extracted from the raw data and extended using antisymmetric lateral continuation (Baranov, 1975, p.42) to a $512 \times 512$ size suitable for processing using, the Fast Fourier transform. After low-pass filtering, to eliminate aliasing, and tapering at the grid boundaries, resampling produced a $64 \times 64$ grid for computations. Due to possible edge effects, only the central $56 \times 56$ area is considered below.

Figure 4.5 shows the resulting contoured field. The major feature present is the northeasterly trending low, up to $50 \mathrm{~km}$ wide and with values up to $-300 \mathrm{nT}$, coinciding with the Grenville front. Accompanying this feature i's a distinct change in character of the field between the two provinces: the Grenville exhibits a highly variable field with 
G - Granvilia Front
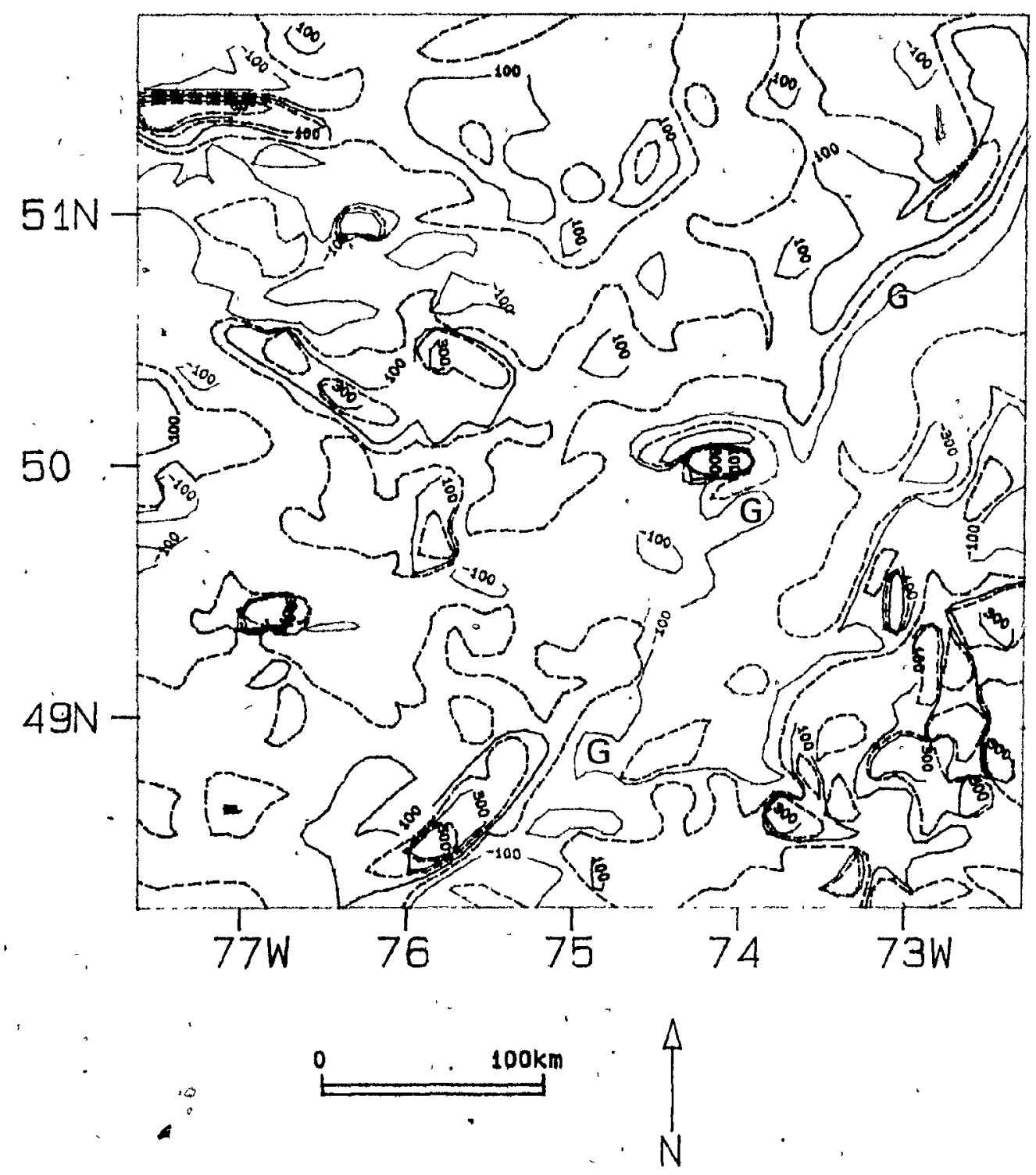

Figure 4.5 Residual aeromagnetic field over the Abitibi-Grenville region. Contour interval is $100 \mathrm{nT}$. 
anomalies of consistently similar extent and magnitude with no perferred direction. The superior province shows large magnetically quiet regions" superposed with small areas of high field intensity and large horizontal gradients. The latter can be correlated with mafic and ultramafic intrusives in the area. Northeasterly trending linear features attributable to diabase dykes are apparent throughout the superior Province (these are more easily seen on the 1:1000 000 scale colour maps of the GSC). The grid size and contour interval of Figure $4.5^{\circ}$ surpress these features. The granite plutons seen from the gravity data usually show a homogeneous magnetic expression although their boundaries may show high magnetic relief due to magnetite enrichment (Schwarz et al, 1984).

The aim of this section is to map lateral variations in the lower boundary of the 'magnetic crust' by modelling the longer wavelength components of the observed residual field. Based on studies of the magnetic properties of mantle derived rocks, the upper mantle appears to be essentially non-magnetic (Wasilewski et al, 1979). Thus, where the Curie isotherm occurs below the Moho (in areas of low geothermal gradient), the boundary between magnetic and non-magnetic material coincides with the Moho. Similarly, in areas of higher geothermal gradient, the long wavelength part of the observed field may, in part, reflect lateral variations of the Curie isotherm within the Earth's crust. Studies of 
crustal magnetic material suggest an increase in magnetization with depth. Wasilewski and Padovani (1980). point out that susceptibility is enhanced at temperatures close to the curie point of magnetic minerals and viscous magnetization generally increases with temperature. These two effects and the likely decrease in remanent magnetization with increased temperature (and hence depth) both lead to an increase in the effective magnetization of the lower crust in the direction, of the Earth's field.

Before attempting inversion, the magnetization contrast at the bottom of the magnetic crust must be specified. Various workers have estimated the value of lower crustal magnetization (summarised by Wasilewski and Mayhew (1982)) based on the modelling of deep crustal layers and agree that the magnetization is large, being in the range $2-6 \mathrm{~A} / \mathrm{m}$. These values coincide with those inferred from equivalent layer magnetization models derived, from satellite magnetometer data (e.g. Mayhew, 1982).

A magnetization 'óf $5 \mathrm{~A} / \mathrm{m}$ and a mean interface depth of $33 \mathrm{~km}$ was chosen for the study area. The effective magnetization was assumed to be in the present day main field direction which has an average inclination of $75^{\circ}$ and declination of $20 \% \mathrm{~W}$ over the area. Figure 4.6 shows the calculated relief for such an interface. "Five iterations were required until the change in RMS error was less than $1 \%$ of the previous value. The Grenville front corresponds to a 


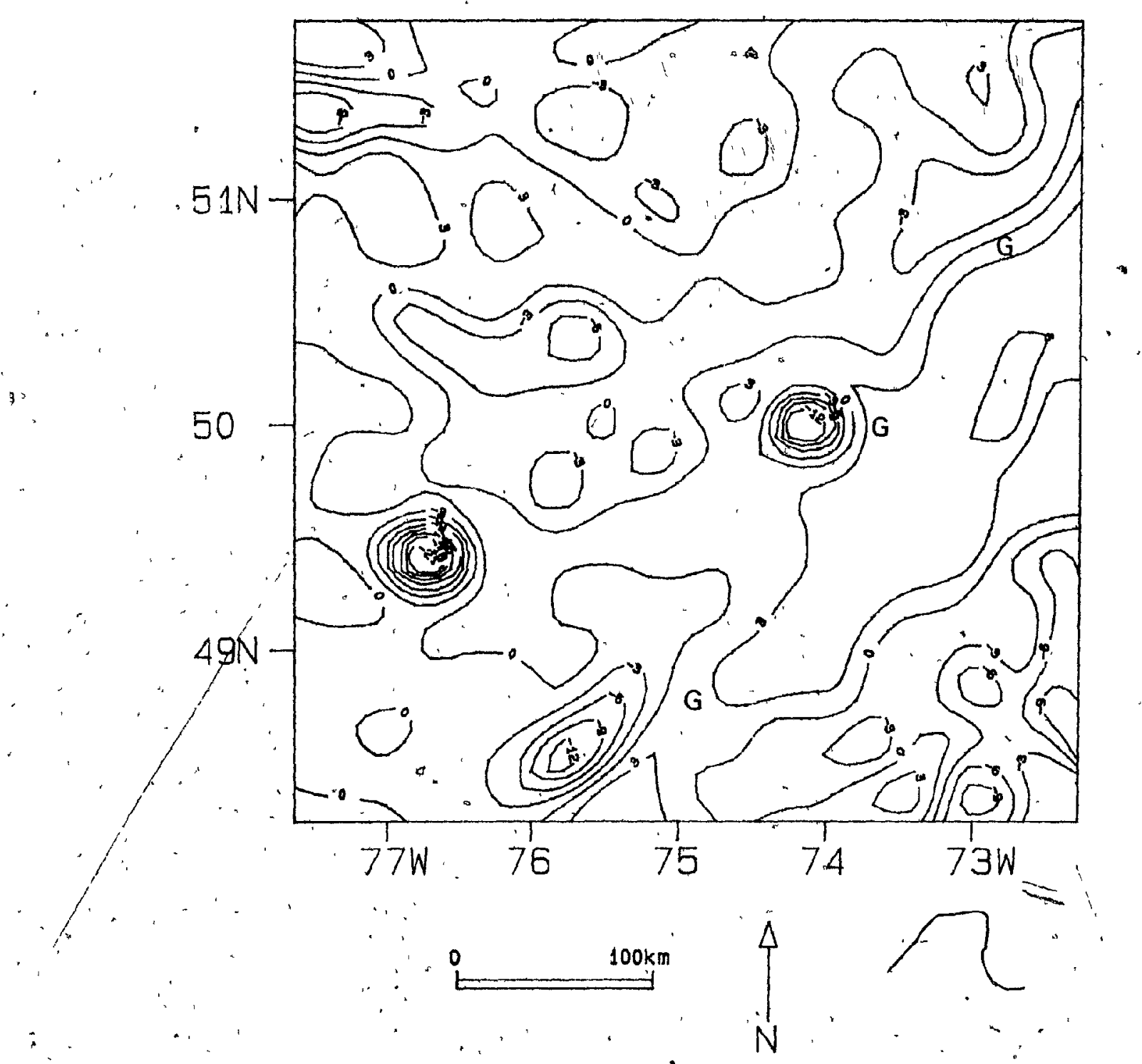

Figure 4.6 Relief of magnetic crust bottom. Contour interval is $3 \mathrm{~km}$. 
thinning of the magnetic crust while an apparent thickening occurs in the Grenville Province to the south east. This is in agreement with the results of part of the 1982 COCRUST seismic experiment (Merey et al, 1985) conducted across the Grenville front. The two circular negative features are the result of the high intensity anomalies seen on the total field map. In the inversion these highs are effectively modelled as point sources becausel the average layer depth is much larger than the true source depth. Aside from such anomalous features, the magnitude of variation in topographic relief for the magnetic case agrees well with that inferred from the gravity data. Figure 4.7 shows the field tomputed from the model in Figure 4.6. The anomalies corresponding to the small highs of Figure 4.5 have been smoothed due to the large source depth of the postulated model. As in the gravity case, the computed field, can be regarded as a Eirst approximation to the regional field in this particular area.

\section{$\underline{4} . \underline{2}$ Conclusion and discussion}

A cost-effective and accurate interpretation method, suitable for use on large gridded potential field data sets has been presented. It is primarily intended for the determination of basement topographies and is developed within the framework of discrete linear inverse theory. 


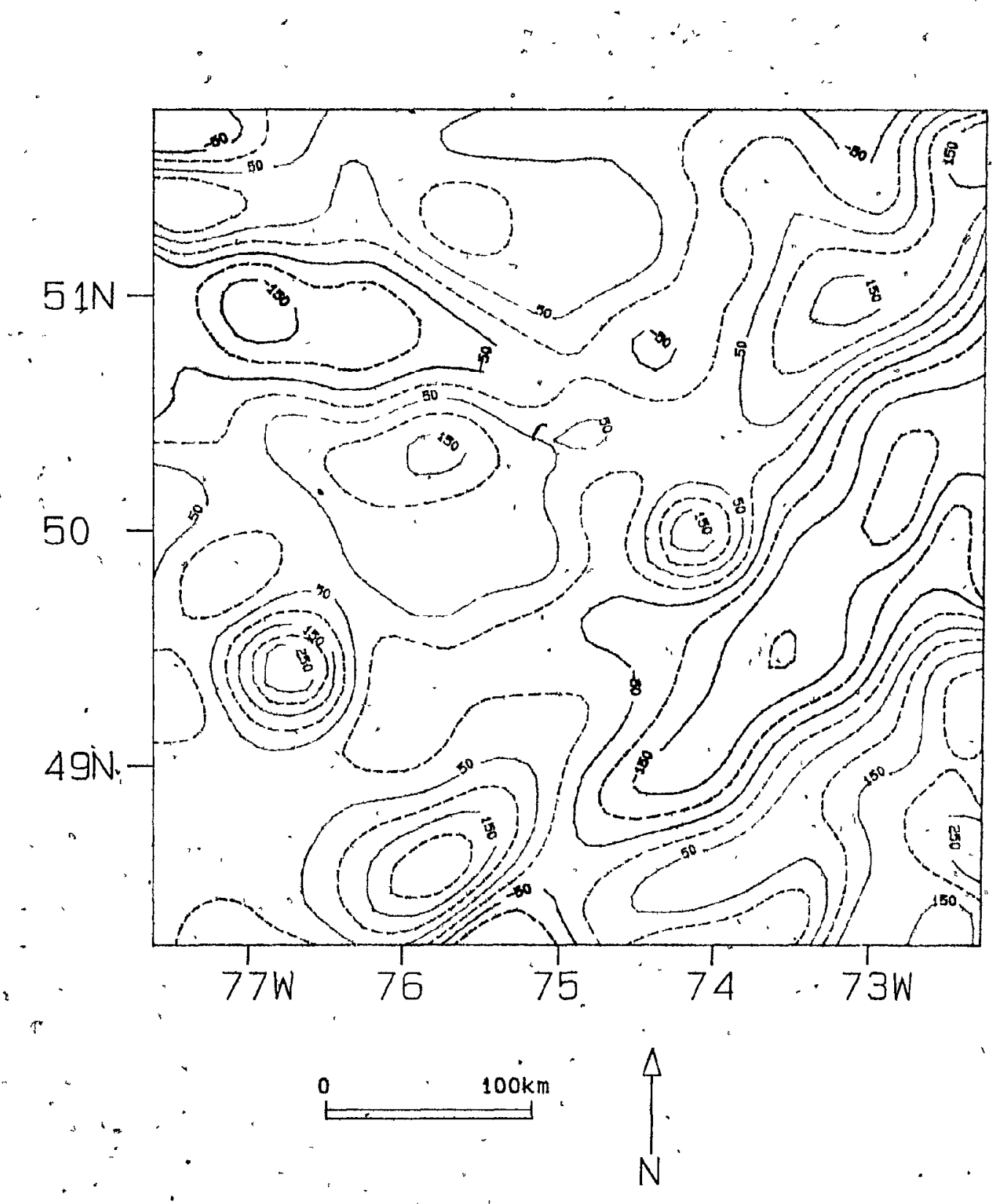

Eigure 4.7 Total field produced by the model of Figure 4.6 . Contour interval is 50nT. 
Hence it offers quantitative measures, of precision and accuracy for the computed solution. Since problem TI suffers from non-uniqueness arising from inaccurate specification of average layer depth and magnetization, the ability of the method to efficiently and inexpensively generate a family of acceptable models is a major advantage over existing techniques.

Computational costs, in terms of storage and operations, have been kept to a minimum by the adoption of Parker's (1973) forward algorithm for the calculation of potential fields caused by one or several layers each with a constant magnetization or density contrast. Additionally, the requirement of standard nonlinear inversion programmes for costly evaluation of partial derivatives and matrix inversion has been obviated by the use. of a constant "Jacobian throughout the iterative procedure. The structure of the Jacobian (it is circulant) allows available rapid algorithms, for Fast Fourier transformation to be used for its decomposition into eigenvalues and eigenvectors. Tests with numerically determined singular value decompositions at each, iteration 'show the proposed method compares favourably. Examination of the one layer eigenvalue spectrum highlights the source of high wavenumber instability due to the presence of small eigenvalues and long wavelength instability due to a zero eigenvalue occurring at zero wavenumber.

Methods for quick evaluation of partial derivatives (if 
required) and residuals are outlined in Chapter 2. In . Chapter 3, a method for determining the degree of damping to be used in inversion is given based on a priori estimation jf the maximum relief variation expected. A trial and error procedure for bounding the layer depth or magnetization when one of these parameters i's known was developed using the RMS error of the first linear approximate topography (FLAT). The feasibility of spectral methods for independent determination of depth and magnetization was investigated in terms of the spectral and statistical properties of relief. functions. Añalysis showed that spectral tẹchniques can be used effectively only when basement reliefs are of small amplitude and predominantly high wavenumber content.

Tests with synthetic data have shown that the algorithm is stable in the presence of long wavelength and random, white noise although the resulting estimates are degraded. The degree of error depends on the spectral properties of the noise and its relation to the passband defined by damping. violating the assumption of a constant magnetization over the layer interface still allows useful structural information to be determined from the data. However,' as the correlation between the magnetization distribution and relief increases, the resulting estimates should be treated with caution. Within the constraints imposed by using the single interface model, the degree of uniqueness of the computed solutions, assuming depth and 
magnetization parameters are correctly chosen, has been demonstrated when inversion is initialised with widely differing starting models that are far from the true relief. In section (3.5) the possibility of extracting parameter resolution measures without matrix inversion has been outlined and shown to provide useful information concerning constructed solution averages.

One layer inversion has been extended to the multi-layered case, for which it is shown that an explicit singular value decomposition exists. Consequently, the speed of the one layer algorithm, can be exploited for multi-layered models. The multi-layer decomposition of 'section (2.7), particularly' the reparameterization, clearly illustrates the problems faced in dealing with such a model: Reparameterizaton is essentially a high-pass filtering for the upper layer and a low-pass filtering for the lower, which, agrees with the accepted result that the low wavenumber content of potential fields increases as source depth increases. Inversion tends to produce estimates with smooth, attenuated lower layer. reliefs with the high wavenumber components being partitioned to the upper layer. Surprisingly, the upper layer is more liable to high wavenumber instability during inversion than the lower layer; this is in contrast to the one layer problem where an increase in layer depth is associated with an increase in instability. 
The effect of the multi-layer linverse of equation $(2.7 .2 .10)$ for the case of two layers is shown to be equivalent to wiener filtering of the data followed by the application of the one layer inverse. The 'signal' and 'noise' 'spectra defining the wiener filter are given by the square of the eigenvalue matrices for the corresponding one layer problem. By. this analogy, it follows that if the theoretical spectra do not match those of the problem, poor relief estimates will result from the proposed method. Synthetic data tests with model kayers exhibiting various spectral properties support this reasoning. The two-layer algorithm produces the most accurate-fesults when the upper layer is composed of predominantly short wavelengths and the lower, of. long wavelengths: The non-uniqueness of the multi-layer problem when applied to the case of a magnetic layer embedded in a nonmagnetic medium is compounded by the existence of layers (whose top and bóttom surfaces can show a high degree of correlation) that produce no observed Eield.

In order to reduce non-uniqueness in, solutions when independent information is available concerning layer depths at given points,. both the one and two-layer algorithms have been extended to incorporate lịnear equality constraints at the expense of a minor increase in computation time". The consequences of these constraints on parameter resolution and reliability have been investigated. 
Since the uniqueness of the one layer solution is guaranteed, constraint information is of use primarily in checking the validity of auxiliary parameters and the single interface model. For the two layer model it was hoped that the introduction of constraints would remove the problem of non-uniqueness arising from reparameterization. Unfortunately this has not been achieved: the adoption of the flat starting model (necessary for the simple computational form of the multi-layer singular value decomposition) renders the method applicable only to those structures whose relief spectra are close to spectra produced by a random distribution of dipoles occuring at the average layer depths.

$\underline{4} \cdot \underline{3}$ Claims to originality

$\because 4$

1. Although numerous methods exist for the determination of crystalline basement topographies from aeromagnetic data; the proposed method is the first to exploit the quasi-linearity of problem TI, resulting in a nonlinear approach consisting of a series of linear problems that are solved in a highly rapid fashion, using Fast Fourier transform techniques. Contrary to standard Marquardt-type algorithms, the determination of an appropriate damping factor is effected prior to inversion, based on a simple criterion: the maximum expected variation in basement 
207

relief.

2. An explicit form of the singular value decomposition is shown to exist for the partial derivative matrix for a model consisting of two or more constantly magnetized layers. An algorithm using this decomposition has been developed in an attempt to solve the multilayer inverse problem for potential fields.

3. Both the one and two-layer cases have been extended to - include linear equality constraints in the form of known layer depths at given points. It is shown that this causes only a minor increase in computation time.

0 


\section{REFERENCES}

Aki, K., Christoffersson, A:, and Husebye, E,S., 1977, Determination of the three-dimensional structure of the lithosphere: J. Geophys. Res., v.82, p.277-296.

Aki, K., and Richards, 'P., l980, Quantitative seismology Theory and methods V.2: W.H. Freeman \& CO..

Albert, A.A., 1972, Regression and the Moore-Penrose pseudoinverse: Academic Press.

Backus, G., and Gilbert, F., 1967, Numerical application of a formalism for geophysical inverse problems: Geophys. J. Roy. Astr. SOC., v.13, p.247-276.

Backus, G., and Gilbert, F., 1968, The resolving power of gross earth data: Geophys. J. Roy. Astr. Soc., v.16, p.169-205.

Backus, G., and Gilbert, F., 1970, Uniqueness in the inversion of inaccurate gross earth data: Phil. Trans. Roy. Soc., v.266A, p.123-192.

Baldi, P., 1981, New aspects in the inversion of gravity profiles by polynomial method: Geophys. Prosp., v.29, p. 426-431.

Baldi, P., and Unguendoli, M., 1978, Inversion of gravity profiles by polynomial method: Geophys. - Prosp., v.26, p. 247-251.

Baranov, W., 1975, Potential fields and their transformations in Applied Geophysics: Gebrueder Borntraeger.: (Geoexploration monograph No. 6)

Barraclough, D.R., Harwood, J.M., Leaton,.B.R., and Malin, S.R.C., 1975, A model of the geomagnetic field at epoch. 1975: Geophys..J. Roy. Astr. Soc., v.43, p.645-659.

Beck, J.V., and Arnold, K.J., 1977, Parameter estimation in engineering and science: John Wiley \& Sons.

Bhattacharyya, B.K., 1966, Continuous spectrum of the total magnetic field anomaly due to a rectangular prismatic body: Geophysics, v. 31, p.97-121.

Bhattacháryya, B.K., and Leu, L-K., 1975, Spectral analysis of gravity. and magnetic anomalies due to two-dimensional structures: Geophysics, v.40, p.993-1013. 
Bott, M.H.P., 1960, The use of rapid digital computing methods for direct gravity interpretation of sedimentary basins: Geophys. J." Roy. Astr. Soc., v.3, p.63-67.

Bott, M.H.P., 1973, Inverse methods in the interpretation of magnetic and gravity anomalies, in B. Alder, Ed.,. Methods in computational physics, v.13: Academic press.

Bullard, E.C., and Cooper, R.I.B., 1948, The determination of the masses necessary to produce a given " gravitational field: Proc. Roy. Soc. Lond., v.i94A, p. 332-347.

Burkhard, N., and Jackson, D. , 1976, Application of stabilized linear inverse theory to gravity data: $J$. Geophys. Res., v.81, p.1513-1518.

Businger, P.A., 1970, Updating a singular valué decomposition: BIT, v.11, p.3.76-385.

Clayton, R.W., and Wiggins, R.A., 1976, Source shape estimation and deconvolution of teleseismic bodywaves: Geophys. J. Roy. Astr. Soc., v.47, P.151-177

Cline, R.E., 1964, Representations'for the generalized inverse of a partitioned matrix: $J$. Soc. Indust. Appl. Math., v.12, p.588-600. .*

Conte, S.D., and DeBoor, C., 1980, Elementary numerical, anaysis: McGraw Hill.

Cooke, D.A., and Schneider, W.A., 1983, Generalized linear inversion of reflection seismic date: Geophysics, v.48, p. $665-676$.

Cordell, L., and Grauch, V.J.S., 1982, Recincilliation of the discrete and integral fourier transforms: Geophysics, v.47, p.237-243.

Courtillot, V., Ducruix, J., and LeMouel, J.L., 1974, A solution of some inverse problems in geomagnetism and gravimetry: J. Geophys. Res., v.79, p.4933-4940.

Crossley, D.J., and Reid, A.B., 1982, Inversion of gamma-ray data for element abundances: Geophysics, v.47., p.117-126. .

Dean, W.C., 1958, Frequency analysis for gravity and magnetic interpretation: Geophysics, v.23, p.97-127. 
Dennis, J.E., 1977, Nonlinear least squares and equations in D. Jacobs, Ed., The state of the art in numerical analysis: Academic Press.

Dyrelius, D., and Vogel, A., i972, Improvement in convergency in iterative gravity interpretation: Geophys. J. Roy. Astr. Soc., v.27, p.195-205.

Franklin, J.N., 1970, Well-posed stochastic extensions of ill-posed linear problems: J. Math. Anal. Appl., v.31, p.682-716.

Gerard, A.: 'and Debeglia, N., 1975, Automatic three dimensional modelling for the interpretation of gravity or magnetic anomalies: Geophysics, v.40, p.1014-1034.

Gill, P.E., and Murray, W.M., 1977, Matrix factorizations in D. Jacobs, Ed., The state of the art in numerical analysis: Academic Press.

, Gill, P.E., Murray, 'W.M., and Wright, M.W., 198I, Practical optimization: Academic Press.

Goldflam, P., Menzel, H., and, Szelwis, R., 1977, Interpretation of gravity data with a periodical structure: Geoexploration, v.15, p.155-161.

Goodwin, A.M., and Ridler, R.H:, 1970, The Abitibi orogenic belt in A.J. Baer, Ed., Precambrian basins and geosynclines of the Canadian shield: Geol. Surv. Can. Paper 70-40, p.1-30.

Graybil1, F.A., 1983, Matrices with applications in statistics: Wadsworth.

Gubbins, D., 1984, Geomagnetic field analysis- II. Secular variation consistent with a perfectly conducting core: Geophys. J. Roy. Astr. Soc., v.77, p.753-766.

Gunn, P.J., 1975, Linear transformations of gravity and hagnetic fields: Geophys. Prosp., v.23, p.300-312.

Gunn, P.J., 1976, Direct mapping of interfaces and thicknesses of layers using gravity and magnetic data: Geoexploration, v.14, p.75-80.

Gunn, P.J., 1979, Examples of advanced computer based magnetic interpretation methods used as routine exploration aids: Bull. Aust. Soc. Explor. Geophys., v.10, p.15-25. 
Gupta, V.K., and Ramani, N., 1980, Some aspects of regional-residual separation of gravity anomalies in a Precambrian terrain: Geophysics, v.45, p.1412-1426.

Hahn, A., 1965, Two applications of Fourier's analysis for the interpretation of geomagnetic anomalies: J. Geomag.0 Geoelec., v.17, p.195-225.

Hahn, A., Kind, E.G., and Mishra, D.C., 1976, Depth estimation. of magnetic sources by Fqurier amplitude spectra: Geophys. Prosp., v.24, p.287-308.

Hartmann, R.R., Teskey, D.J., and Friedberg, J.L.., 1971, A system for rapid digital aeromagnetic interpretation: Geophysics, v. 36, p.891-918.

Hinze, W.J., 1979, Continental magnetic anomalies: Rev. Geophys. Space Phys., v.17, p.257-273.

Hoerl, A.E., and Kennard, R.W., 1970, Ridge regression: biased estimation for non-orthogonal problems: Techonometrics, v.12, p.55-67.

Ilkisik, O.M., and Jones, A.G., 1984, Statistical evaluation of MT and AMT methods applied to a basalt covered area in southeastern Anatolia, Turkey: Geophys. Prosp., v.32, p.706-724.

Inman, J.R., 1975, Resistivity inversion with ridge regression: Geophysics, v.40, p. 798-817.

Jackson, D.D., 1972, Interpretation of inaccurate, insufficient and inconsistent data: Geophys. J. Roy. Astr. Soc., v.28, p.97-109.

Jackson, D.D., 1979, The use of a priori data to resolve non-uniqueness in Iinear inversion: Geophys. J. Roy. Astr. Soc., v.57, p.137-157.

Jain, S., 1976, An automated method of direct interpretation of magnetic profiles: Geophysics, v.41, p.531-541.

Jupp, D.L.B., and Vozoff, K., 1975, Stable iterative methods for the inversion of geophysical data: Geophys. J. Roy. Astr. Soc., v.57, p.137-157.

Kanasewich, E.R., 1981, Time sequence analysis in geophysics: University of Alberta Press.

Karner, G.D., and Watts, A.B., 1983, Gravity anomalies and flexure. of the lithosphere at mountain ranges: $J$. Geophys. Res., v.88, p.10449-10477. 
Kilty, K.T., 1983, Werner deconvolution of profile potential field data: Geophysics, v.48, p.234-237.

$\mathrm{Ku}$, C.C., 1976, Numerical inverse magnetotelluric problems: Geophysics, v.4I, p.276-286.

Ku, C.C., and Sharp, D., 1983, werner deconvolution for automated magnetic interpretation and its refinement using Marquardt's inverse modelling: Geophysics, v.48, p. $754-774$.

Kunuratnam, K., 1972, An iterative method for the solution of a nonlinear inverse problem in magnetic interpretation: Geophys. Prosp., v.20, p.439-437.

Lanczos, C., 1963, Linear differential operators: D. Van Nostrand Co..

Lawson, C.L., and Hanson, R.J., 1974, Solving least squares problems: Prentice Hall.

Lehmann, H.J., 1970, Examples for the separation of fields of magnetic sources in different depths by the harmonic analysis method: Boll. Geofis. Teor. Appl., v.12, p. 97-112.

Levepberg, G., 1944, A method for the solution of certain - nonlinear problems in least squares: Quart. Appl. Math., v.2, p.164-166.

Luddèn, J.N.Y and Hubert, C., 1984, Evidence for the juxtaposition of contrasting terranes in the Abitibi belt: Paper presented at GAC-MAC Meeting, London, Ontario, May.

Mayhew, M.A., 1982 , An equivalent layer magnetization model for the United States "derived from satellite altitude magnetic anomalies: J. Geophys. Res., v.87, p. 4837-4845.

Marquardt, D.W., 1963, Án algorithm for least squares estimation of nonlinear parameters: J. Soc. Indust. Appl. Math., v.ll, p.431-44l.

Marquardt D.W., 1970, Generalized inverses, ridge regression, biased linear estimation and nonlinear estimation: Techonometrics, v.12, p.591-612.

Mereu, R.F., and Jobidon, G., 1971, A seismic investigation of the crust and Moho on a line perpendicular to the Grenville Front: Can. J. Earth Sci., v.8, p.l553-1583. 
Mereu, R.F., Wang, D., Kuhn, O., Forsyth, D.A', Green, A.G., Morel, P., Buchbinder, G.G.R., Crossley, D., Schwarz, E., duBerger, R., Brooks, C., and Clowes, R., 1985, The 1982 COCRUST long range seismic experiment across the Ottawa-Bonnechere Graben and Grenville Front in Ontario and Quebec: Paper submitted to Geophys. J. Roy. Astr. Soc..

Mishra, D.C., and Pedersen, L.B., 1982, Statistical analysis of potential fields from subsurface reliefs: Geoexploration, v.19, p.247-265.

Mishrà, D.C., 1984, Magnetic anomalies - India and Antarctica: Earth Planet. Sci. Lett., v.71, p.173-180.

Nabighian, M.N., 1972, The analytical signal of two-dimensional magnetic bodies with polygonal cross-section: its properties and use for automated anomaly interpretation: Geophysics, v.37, p.507-517.

Nabighian, M.N., 1974, Additional comments on the the analytical signal of two-dimensional magnetic bodies with polygonal cross-section: Geophysics, v.39, p. $85-92$.

Nabighian, M.N., 1984, Toward a three dimensional automatic interpretation of potential field data via generalised Hilbert transforms: Geophysics, v.49, p.780-786.

Naudy, H., 1971, Automatio determination of depth on aeromagnetic profiles: Geophysics, v.36, p.717-722.

Nettleton, L.L., 1'976, Gravity and magnetics in oil prospecting: McGraw Hill.

Noble, B., 1976, Methods for computing the Moore-Penrose generalized inverse and related matters in M. $\mathrm{Z}$. Nashed Ed., Generalized inverses and applications : Academic Press.

Nolet, G., 1978, Simultaneous inversion of seismic data: Geophys. J. Roy. Astr. Soc., v.55, p.679-691.

O'Brien, D., 1972, Compudepth. A new method for depth to basement computation. Paper presented at Society of Exploration Geophysicists, 42 nd meeting, Anaheim, California., November 29, 1972.

Oldenberg, D.W.' 1974, The inversion and interpretation of gravity anomalies: Geophysics, v.39, p.526-536. 
Parker, C.I., 1984, Crustal structure of the Abitibi greenstone belt determined from refraction seismology: M.Sc. Thesis, McGill University, Montreal.

Parker, R.I., 1973, The rapid calculation of potential anomalies: Geophys. J. Roy. Astr. Soc., v.3I, p. 447-455.

Parker, R.L., and Huestis, S.P., 1974, The inversion of magnetic anomalies in the presence of topography: $J$. Geophys. Res., v.79, p.1587-1593.

Pedersen, L.B., 1977, Interpretation of potential field data - A generalized inverse approach: Geophys. Prosp., v.25, p.799-230.

Pedersen, L.B., 1978, A statistical analysis of potential fields using a vertical circular cylinder and a dyke: Geophysics, v.43, p.943-953.

Penrose, R., 1955, A generalized inverse for matrices: Proc. Camb. Philos. Soc., v.51, p.406-413.

Percival, J.A., and Card, K.D., 1983, Archean crust as revealed in the Kapuskasing uplift, Superior Province, Canada: Geology, v.11, p.323-326.

Peters, L.J., 1949, The direct approach to magnetic interpretation and its practical application: Geophysics, v.14, p.290-320.

Quereshi, I.R., and Mula, H.G., 1971, Two-dimensiónal mas distributions from gravity anamalies: a computer method: Geophys. Prosp., v.19, p.180-191.

Rodgers, C.D., 1976, Retrieval of atmospheric temperature profiles from remote measurements of thermal radiation: Rev. Geophys. Space Phys., v.14; p.609-624.

Roy, A.A., 1966, The method of continuation in mining gephysical interpretation: Geoexploration,' v.4, p. $65-83$.

Schwarz, E.J., Laverdure, L., Losier, L.; and Poterlot, E. . 1984, Preliminary. gravity, magnetic and refraction seismic results from the Abitibi belt, Quebec in Current Research, Part A: Geol. Surv. Can. Paper, 84-1A, p. 239-246.

Skeels, D.C., 1947, Ambiguity in, gravity interpietat on: Geophysics, v.12, p.43-56. 
Solovyev, O.A., 1962, Use of the frequency method for the determination of some parameters of magnetic bodies: Izv. AK. Nauk. Sibirskoye. Ot. Geologika i Geofizika 2,122 .

Spector, A., and Grant, F.S., 1970, Statistical methods for interpreting aeromagnetic data: Geophysics, v.35, p. 293-302.

Spector, A., and Parker, w., 1979, Computer compilation and interpretation of geophysical data in P.J. Hood, Ed., Geophysics and geochemistry in the search for metallic ores: Geol. Surv. Can. Econ. Geol. Rep. 31, p.527-544.

-Spedicato, E., 1980, Variable "metric methods for unconstrained optimization in Dixon, L.C.W. et al, Eds., Nonlinear optimization - theory and algorithms: Birkhauser.

Sprenke, K.F., and Kanasewich, E.R., 1982, Gravity modelling and isostasy in western Canada: J. Can. Soc. Expl. Geophys., v.18, p.49-57.

Tanner, J.G., 1967, An automated method of gravity interpretation: "Geophys. J. Roy. Astr. Soc., v.13, p. 339-347.

Telford, W.M., Geldart, L.P., Sheriff, R.E., and Keys, D.A., I976, Applied geophysics: Cambridge University Press.

Teskey, D.J., 1978, Design of a semi-automated three dimensional interpretation system for potential field data: Ph.D. Thesis, McGill University, Montreal.

Thompson, D.W., 1982, EULDPH: A new technique for making computer assisted depth estimates from magnetic data: Gèophysics, v.47, p. 31-37.

Treitel, S., Clement, W.G., and Kaul, R.K., 1971, "The spectral determination of depths to buried magnetic basement: Geophys. J. Roy. Astr. SOC.,"v.24, p. $415-428$.

Troshkov, G.A., and Shalaev, S.V., 1961; Application of the Fourier transform to the solution of the reverse problem of gravity and magnetic surveys: Translated in J. Can. Expl. Geophy. April 1968, p.48-62.

Twomey, S., 1977, Introduction to the mathematics of inversion in remote sensing and indirect measurements: Elsevier. 
Wasilewski, P.J., Thomas, H.H., and Mayhew, M.A., 1979, The Moho as a magnetic boundary: Geophys. Res. Lett., v.6, p. 541-544.

Wasilewski, P.J., and Padovani, E., 1980, Magnetization in the lower crust (abstract): Eos, v.61, p.222.

Wasilewski, P.J., and Mayhew, M.A., 1982, Crustal xenolith magnetic properties and long wavelength anomaly source requirements: Geophys. Res. Lett., v.9, p.329-333.'

Werner, S., 1953, Interpretation of magnetic anomalies of sheet-like bodies: Sveriges Geologiska Undersokning Ser.e., no.508.

Wiggins, R.A., 1972, The general linear inverse problem: Implications of surface waves and free oscillations for earth structure: Rev. Geophys. Space Phys., v.10, p. 251-285.

Wiggins, R.A., Larner, K.., and Wisecup, R., 1976, Residual statics, as a general linear inverse problem: Geophysics, v.41, p.922-938. 